\title{
Measurement of Neutral B Meson Mixing in Electron-Muon Events in 1.8-TeV Proton-Antiproton Collisions
}

\author{
January 1996
}

\section{Hisafumi Mitsushio}

A dissertation submitted to the Doctoral Program in Physics, the University of Tsukuba in partial fulfillment of the requirements for the degree of Doctor of Philosophy (Science) 


\begin{abstract}
The $B^{0} \bar{B}^{0}$ mixing parameter, $\chi$, has been measured in $e \mu$ events in proton-antiproton collisions at a center of mass energy of $1800 \mathrm{GeV}$. The experiment has been performed at the Fermi National Accelerator Laboratory in the United States using the Tevatron accelerator. The data were collected with the Collider Detector at Fermilab (CDF) during the 1992-1993 Tevatron collider run. The corresponding integrated luminosity is $20 \mathrm{pb}^{-1}$.

The $B^{0} \bar{B}^{0}$ mixing is a process by which neutral $B$-mesons oscillate into their antiparticles $\left(B^{0} \leftrightarrow \bar{B}^{0}\right)$. In the absence of mixing, the direct semileptonic decay of a $b \vec{b}$ pair produced in $p \bar{p}$ collisions results in a pair of leptons with opposite charge. The $B^{0}$ or $\bar{B}^{0}$ may undergo mixing, resulting in a like-sign (LS) lepton pair. The magnitude of mixing is determined from the ratio of like-sign dilepton pairs to opposite-sign (OS) dilepton pairs. In this thesis, electron-muon pairs were used as the dilepton pairs.

The CDF detector is a general purpose detector built to explore $p \tilde{p}$ collisions. It covers almost the full solid angle around the $p \bar{p}$ interaction point. The CDF detector consists of tracking detectors and calorimeters for measurements of the particle momentum and energy. In order to identify muons, muon detectors are installed at the outside of the detector. Electrons, photons, muons, neutrinos, hadrons and jets are identified using these detector components.

We describe identification of muons and electrons, and then event selection of $b \bar{b}$ events. To remove events in which both leptons are produced by one $B$ meson, it is required that an opening angle of the electron and muon is large enough. We apply the requirement that the transverse momentum of leptons to jet axis, $P_{T}^{\text {rel }}$, is large enough for $b \bar{b}$ events. This requirement significantly reduces background and systematic uncertainty. 1710 opposite-sign and 861 like-sign $e \mu$ events are found after the event selection.

Then the estimation of the background is described. We estimate the fraction of fake $e \mu$ events using $J / \psi, K_{s}$ and $\phi$ samples, minimum bias data and Monte Carlo simulation. Most of the background for $e \mu$ events are pairs of real-electron and fake-muon. The main source of the fake muons is decay-in-flight of hadrons. The systematic uncertainty in estimating the fake muon fraction is substantially reduced by using a newly installed muon detector.

Like-sign lepton pairs are also produced by semileptonic sequential decays of $B^{0}$ or $\bar{B}^{0}$ mesons. The fraction of sequential decays is estimated from Monte Carlo $b \bar{b}$ events by taking into consideration the uncertainties in the structure functions and fragmentation functions. The fraction of direct $c \bar{c}$ production is estimated directly from data by fitting the Monte Carlo $P_{T}^{\text {rel }}$ distribution of leptons from direct and sequential decays as well as from $c \bar{c}$ production to the observed spectra.
\end{abstract}

From the above analysis, we obtain

$$
\chi=0.130 \pm 0.010(\text { stat. }) \pm 0.010(\text { syst. })
$$


This result improves the previous CDF result by a factor of 3 and is one of the most accurate measurements of $\chi$ at the present time. It is consistent with other measurements and also with the expectations from the standard model.

Since both neutral $B$ mesons, $B_{d}^{0}$ and $B_{s}^{0}$, are produced in $p \bar{p}$ collisions, the mixing parameter for $B_{s}, \chi_{s}$, is estimated by using the mixing parameter for $B_{d}$ measured by the ARGUS and the CLEO groups in $e^{+} e^{-}$collisions and by assuming the fractions of $B_{d}^{0}$ and $B_{s}^{0}$ mesons produced in $b \bar{b}$ events. 


\section{Acknowledgements}

I would like to thank my advisor, Professor Koji Takikawa, for his guidance and encouragement throughout my graduate career. I want to thank Professor Kunitaka Kondo who gave me the opportunity to work at CDF and constant support through my graduate student career.

I wish to express my deepest appreciation to Itsuo Nakano and Yoshihiro Seiya who have discussed on physics and other various things with me. I could not have finished this work without their continuous help.

Manfred Paulini, Joe Kroll, Paris Sphicas and Fritz Dejongh gave me a lot of help as the CP-MIXING and B physics group convener. They gave me useful suggestions and chances to present my analysis result at the meetings. Intae Yu and Malie Yin gave me useful information for my analysis. Jonathan Lewis, John Marriner, Phil Schlabach, Tony Liss, Randy Keup, Alain Gauthier, Mark Vondracek, Andrew Martin, Yoshiki Teramoto, Takuo Yoshida and Yukihiro Kato collaborated with me during my detector work. G. P. Yeh and Lingfeng Song gave me useful suggestions and encouragements at Fermilab. Conversations with Luc Demortier, Shuichi Kunori, Shoji Mikamo, Nobuaki Oshima, Susumu Igarashi, Taiji Yamanouchi, Masanori Mishina and Hidemi Akimoto are unforgettable during my stay at Fermilab.

Discussions on physics and other various things with Shinhong Kim, Kazuhiko Hara, Kiyoshi Yasuoka, Shige Miyashita, Takeshi Chikamatsu, Makoto Shimojima, Tomohiro Kaneko and Fumihiko Ukegawa were very beneficial for me. I also wish to thank other members of the Tsukuba high energy physics group for their constant help. They include Takashi Ino, Masahiko Yokoyama, Mikio Takano Nobuyuki Uemura, Shinichi Funaki, Masakazu Okabe, Hiroyuki Sato, Eiichiro Hayashi, Toshiharu Uchida, Tsuyoshi Takano, Mariko Ninomiya, Housai Nakata, Shin Aota, Hirofumi Ikeda, Tomoko Kuwabara, Junichi Suzuki and other colleagues.

I want to express my thanks to Carol Picciolo and Dee Hahn for their secretary works at Fermilab. I thank Japanese secretary Kyoko Kunori who has always been giving us 
kind support at Fermilab. I thank Kazuko Kumashiro and Mutsumi Uenishi for their secretary work at University of Tsukuba.

Members of Ballroom Dance Club of University of Tsukuba gave me a lot of continuous encouragements.

Finally, I would like to thank my parents, sister, brother and grandmother for their continuous support.

We thank the Fermilab staff and the technical staffs of the participating institutions for their vital contributions. This work was supported by the U.S. Department of Energy and National Science Foundation; the Italian Istituto Nazionale di Fisica Nucleare; the Ministry of Education, Science and Culture of Japan; the Natural Sciences and Engineering Research Council of Canada; the National Science Council of the Republic of China; the A. P. Sloan Foundation; and the Alexander von Humboldt-Stiftung. 


\section{Contents}

1 Introduction 1

1.1 Standard Model . . . . . . . . . . . . . . . . . . 1

1.2 Basic formalism of mixing mechanism . . . . . . . . . . . . . 3

2 Mixing in Neutral $B$ Mesons $\quad 7$

$2.1 \quad B$ meson production $\ldots \ldots \ldots \ldots$

$2.1 .1 \quad b \bar{b}$ production ......................... 7

$2.1 .2 \quad b$ quark fragmentation $\ldots \ldots \ldots . \ldots . \ldots . \ldots$

$2.2 B^{0} \bar{B}^{0}$ mixing ........................... 8

2.2.1 Previous measurements of mixing in the $B_{d}^{0}$ and $B_{s}^{0}$ system . . . 13

3 Apparatus $\quad 25$

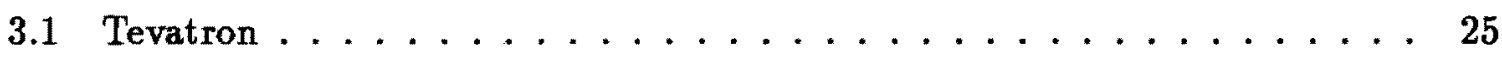

3.2 Collider Detector at Fermilab . . . . . . . . . . . . . 26

3.2 .1 Beam-beam counters . . . . . . . . . . . . . 27

3.2 .2 Tracking detectors .......................... 28

3.2 .3 Calorimeters ...................... 29

3.2 .4 Muon detectors ...................... 32

3.2 .5 Trigger ....................... 34

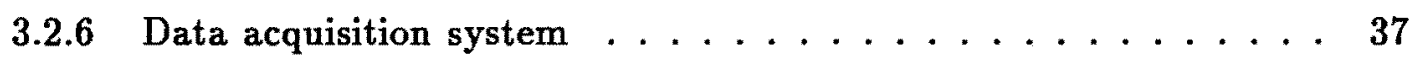

3.2.7 Luminosity measurement . . . . . . . . . . . . . 38 
4 Analysis Outline and Data Reduction 53

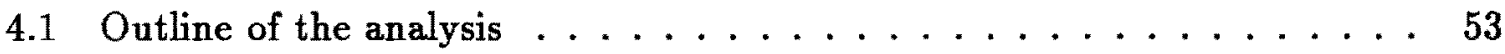

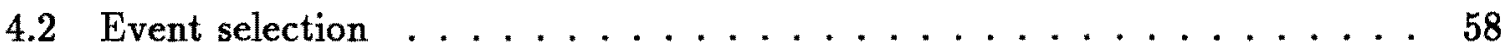

4.2 .1 Muon selection ........................ 58

4.2 .2 Electron selection . . . . . . . . . . . . . . 61

$4.2 .3 b \bar{b}$ event selection $\ldots \ldots \ldots \ldots 7$

4.2 .4 Results of event selection . . . . . . . . . . . . . . 71

5 Backgrounds and Systematic Uncertainties $\quad 96$

5.1 Fraction of fake $e \mu$ events . . . . . . . . . . . . . . 96

5.1 .1 Fake muon fraction . . . . . . . . . . . . . 98

5.1.2 Fake electron fraction .................. 100

5.1 .3 Contribution of photon conversions ............. 103

5.1.4 Fake $e \mu$ fraction $F_{e \mu} \ldots \ldots \ldots \ldots$. . . . . . . . . 104

5.1.5 Asymmetry of fake $e \mu$ events . . . . . . . . . . . . 104

5.2 Fraction of sequential decays . . . . . . . . . . . . . 107

5.2.1 Monte Carlo of $b \bar{b}$ events . . . . . . . . . . . . . . 108

5.2 .2 Estimation of the $f_{s} \ldots \ldots \ldots$. . . . . . . . . . .

5.2.3 Correcting $f_{s}$ for $J / \psi$ and $b \rightarrow \bar{c} \rightarrow l$ events ........ 118

5.2 .4 Systematic uncertainties in $f_{s} \ldots \ldots \ldots \ldots$

5.3 Fraction of direct $c \bar{c}$ background . . . . . . . . . . . . . . 122

5.3.1 Method of $f_{c}$ estimation ................... 122

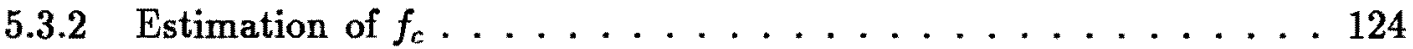

5.3 .3 Systematic uncertainties in $f_{c} \ldots \ldots \ldots \ldots$

6 Results and Discussion $\quad 146$

6.1 Mixing parameter, $\chi \ldots \ldots \ldots \ldots \ldots$

6.2 Mixing parameter for $B_{s}$ mesons, $\chi_{s} \ldots \ldots \ldots \ldots$. . . . . . . 147

7 Conclusion $\quad 153$ 
A Estimation of Probabilities for Real Leptons from $J / \psi \rightarrow l l$ Events

B Uncertainty in $f_{s}$ due to Monte Carlo Statistics, Structure Function and Peterson Parameter 


\section{List of Tables}

1.1 Leptons and quarks of the Standard Model . . . . . . . . . . 2

2.1 Mixing parameter $x \ldots \ldots \ldots \ldots \ldots \ldots \ldots \ldots \ldots \ldots$

3.1 Geometric coverage in $\eta$ direction and absorption thickness for calorimeter components. . . . . . . . . . . . . . . . . 30

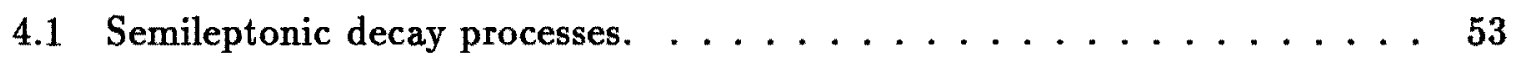

4.2 Charge combinations of $e$ and $\mu$ for each Process. OS (LS) means oppositesign (like-sign). . . . . . . . . . . . . . . . 54

4.3 Probability for each mixing process. . . . . . . . . . . 55

4.4 Other semileptonic decay processes. . . . . . . . . . 55

4.5 Charge combinations of $e$ and $\mu$ for Process 5 and 6. OS (LS) means opposite-sign (like-sign). . . . . . . . . . . . . 56

4.6 Charge combinations of $e$ and $\mu$ for Process 7 and 8. OS (LS) means opposite-sign (like-sign). . . . . . . . . . . . . . 56

4.7 Summary of the $e \mu$ event selection. . . . . . . . . . . 70

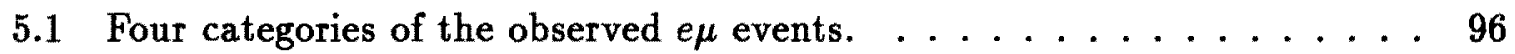

5.2 Summary of the fake lepton fraction estimation. The uncertainties include both the statistical and systematic uncertainties. . . . . . . . 105

5.3 The daughter hadron charge asymmetry for each $B$ meson. The uncertainties are due to the statistics of Monte Carlo events. . . . . . . . 107 
5.4 The numbers of $e \mu$ events which passed the $e \mu$ event selection and the corresponding luminosities. The structure function is MRSD0 $-^{\prime}$ and the Peterson parameter is set to $0.003 . \ldots \ldots \ldots$. . . . . . . 109

5.5 The numbers of $e \mu$ events which passed the $e \mu$ event selection and the corresponding luminosities. The structure function is MRSD0 $-^{\prime}$ and the Peterson parameter is set to $0.006 . \ldots \ldots \ldots \ldots \ldots \ldots$

5.6 The numbers of $e \mu$ events which passed the $e \mu$ event selection and the corresponding luminosities. The structure function is MRSD0 $-^{\prime}$ and the Peterson parameter is set to $0.009 . \ldots \ldots \ldots \ldots \ldots \ldots$

5.7 The numbers of $e \mu$ events which passed the $e \mu$ event selection and the corresponding luminosities. The structure function is EHLQ and the Peterson parameter is set to $0.003 \ldots \ldots \ldots \ldots \ldots \ldots \ldots \ldots$

5.8 The numbers of $e \mu$ events which passed the $e \mu$ event selection and the corresponding luminosities. The structure function is EHLQ and the Peterson parameter is set to $0.006 \ldots \ldots \ldots \ldots \ldots \ldots \ldots$

5.9 The numbers of $e \mu$ events which passed the $e \mu$ event selection and the corresponding luminosities. The structure function is EHLQ and the Peterson parameter is set to $0.009 \ldots \ldots \ldots \ldots \ldots$. . . . . . . . 114

5.10 The numbers of $e \mu$ events which passed the $e \mu$ event selection and the corresponding luminosities. The structure function is DFLM and the Peterson parameter is set to $0.003 . \ldots \ldots \ldots \ldots \ldots \ldots \ldots$

5.11 The numbers of $e \mu$ events which passed the $e \mu$ event selection and the corresponding luminosities. The structure function is DFLM and the Peterson parameter is set to $0.006 . \ldots \ldots \ldots \ldots \ldots$

5.12 The numbers of $e \mu$ events which passed the $e \mu$ event selection and the corresponding luminosities. The structure function is DFLM and the Peterson parameter is set to 0.009 
5.13 The $f_{s}$ for different structure functions where the Peterson parameter

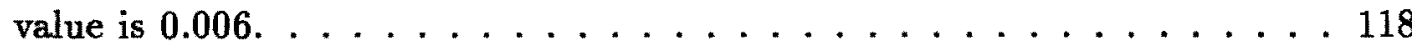

5.14 The numbers of $b \rightarrow J / \psi \rightarrow l$ and $b \rightarrow \bar{c} \rightarrow l$ events which passed the $e \mu$ event selection and the corresponding luminosities, where the structure function is MRSD0 -' and the Peterson parameter is set to $0.006 . \ldots 119$

5.15 The numbers of Monte Carlo events which passed the $e \mu$ selection for OS and LS at the integrated luminosity of $20 \mathrm{pb}^{-1}$. The structure function is MRSD0 $-^{\prime}$ and Peterson parameter is set to $0.006 . \ldots \ldots 120$

5.16 The $f_{s}$ for different values of the Peterson parameter $(\epsilon)$, where the structure function is MRSD0-'. The uncertainties are due to the statistics of the Monte Carlo events. . . . . . . . . . . . . . . . . 121

5.17 The $f_{s}$ for different ratios of gluon splitting events to $b \bar{b}$ direct productions, where the structure function is MRSD0 -' and the Peterson parameter is

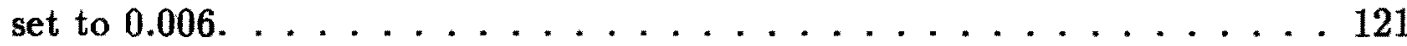

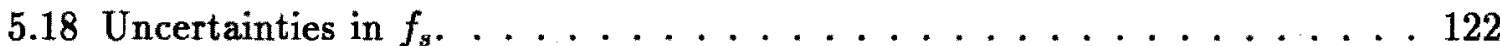

5.19 The $f_{c}$ for different structure functions. . . . . . . . . . 125

5.20 The $f_{c}$ for values of difference between the numbers of fake leptons in OS

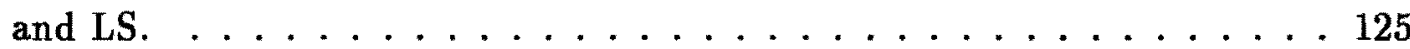

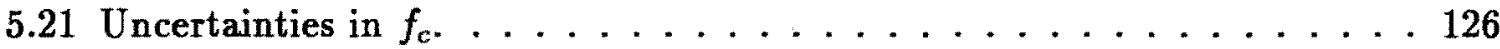

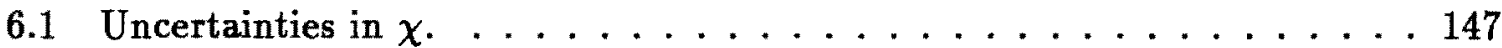

B.1 Fraction of sequential decays with MRSD0-', EHLQ and DFLM 260 structure functions and, with Peterson parameter $\epsilon=0.003,0.006,0.009 .157$ 


\section{List of Figures}

1.1 Feynman diagrams for the $B^{0} \bar{B}^{0}$ mixing process. . . . . . . . 6

2.1 Feynman diagrams for lowest order $b \bar{b}$ production $\ldots \ldots \ldots \ldots$

2.2 The example of Feynman diagrams for next leading order $b \bar{b}$ production . 19

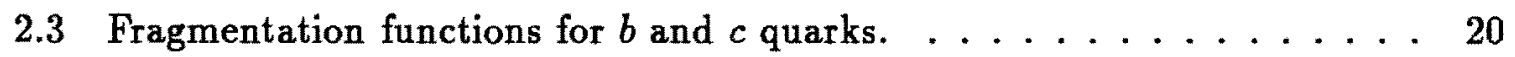

2.4 The solid lines show time dependent probabilities of finding $B^{0}$ and $\bar{B}^{0}$ in an initially pure $B^{0}$ state for $B_{d}$ and $B_{s}$ mesons. The dotted lines show the total decay probabilities. We assume $\Delta M / \Gamma=0.67(5.0)$ for the $B_{d}^{0}$

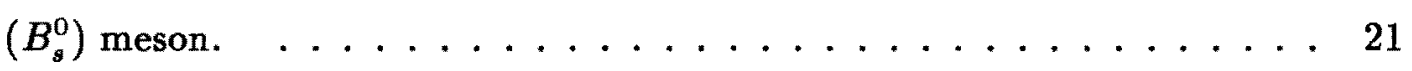

2.5 The completely reconstructed event consisting of the decay $\Upsilon(4 S) \rightarrow B^{0} B^{0} .22$

2.6 $M M^{2}$ spectra for $l^{+} \pi^{-}$(points with error) for events with an additional lepton with momentum $1.4<p_{l}<2.5$ : background (dotted histogram) and the result of the fit (full histogram) $a$ ) for like-sign leptons; $b$ ) for

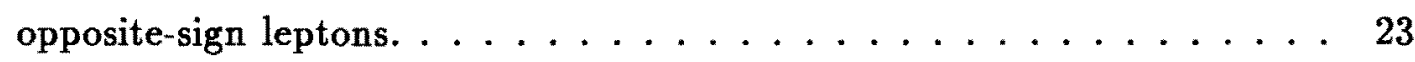

2.7 Efficiency corrected spectrum of additional leptons in tag events, for a) opposite-sign and $b$ ) same-sign leptons. Superimposed are fits (solid) to a primary lepton spectrum (dashed) plus a secondary spectrum (dash-dot). 24

3.1 Diagram of the Tevatron accelerator complex. . . . . . . . . 40

3.2 A perspective view of the CDF detector showing the central detector, the forward and the backward detectors. . . . . . . . . . 41 
3.3 A side-view cross section of the CDF detector. The detector is forwardbackward symmetric about the interaction point. . . . . . . . 42

3.4 Cross-sectional view of the central detectors. . . . . . . . . . . . 43

3.5 A beam's eye view of one of the beam-beam counter planes. . . . . . 44

3.6 A perspective view of the SVX barrel. . . . . . . . . . . . 45

3.7 An endplate of the CTC showing the arrangement of the blocks which hold the 84 layers of sense wires. . . . . . . . . . . . 46

3.8 Hadron calorimeter tower in one of eight identical $\eta-\phi$ quadrants $(\Delta \phi=$ $\left.90^{\circ}, \eta>0\right)$. The heavy lines indicate module or chamber boundaries. The EM calorimeters have complete $\phi$ coverage out to $\eta=4.2$. . . . . .

3.9 Schematic of a wedge module of the CDF central calorimeter showing the coordinate system as measured by the strip chamber used for test beam energy and mapping measurements. The $45^{\circ}$ end plate is in the $+z$ direction; the "left" of the module was defined as being in the $+x$ direction. 48

3.10 Map of muon eta-phi coverage. . . . . . . . . . . . . . 49

3.11 The layout of the central muon chambers in one of the central wedges. . 50

3.12 The arrangement of the four planes of central muon chambers in a view along the beam direction. The drift times $t_{2}$ and $t_{4}$ are used at the trigger level to determine a muon momentum cut off. . . . . . . . . 51

3.13 Schematic view of a CMP-CMX tube $\ldots \ldots \ldots \ldots \ldots 2$

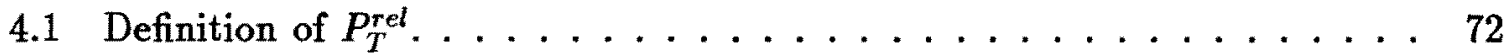

4.2 The distribution of the impact parameter of muon tracks; $D_{0}$. The distributions for muon candidates in the $J / \psi \rightarrow \mu \mu$ events (solid) and those for the jet sample (dotted) are shown. . . . . . . . . . . . . 73

4.3 The distribution of the difference between the $z$ position of event vertex and the $z$ position of muon tracks; $\left|Z_{\text {event }}-Z_{0}\right|$. The distributions for muon candidates in the $J / \psi \rightarrow \mu \mu$ events (solid) and those for the jet sample (dotted) are shown. . . . . . . . . . . . . . . 74 
4.4 The distribution of the number of hits in CTC chamber. The distributions for muon candidates in the $J / \psi \rightarrow \mu \mu$ events (solid) and those for the jet sample (dotted) are shown. . ................ 75

4.5 The distribution of position matching between a stub in the CMU chambers and an extrapolated CTC track to the $\mathrm{CMU} ; \Delta X(C M U)$. The distributions for muon candidates in the $J / \psi \rightarrow \mu \mu$ events (solid) and for the jet sample (dotted) are shown. . . . . . . . . . . . . 76

4.6 The distribution of position matching between a stub in the CMP chambers and an extrapolated CTC track to the CMP; $\triangle X(C M P)$. The distributions for muon candidates in the $J / \psi \rightarrow \mu \mu$ events (solid) and for the jet sample (dotted) are shown. . . . . . . . . . . . 77

4.7 The distribution of $\chi^{2}$ for position matching between a stub in the CMU chambers and an extrapolated CTC track to the $\mathrm{CMU} ; \chi^{2}(C M U)$. The distributions for muon candidates in the $J / \psi \rightarrow \mu \mu$ events (solid) and for the jet sample (dotted) are shown. . . . . . . . . . . . .

4.8 The distribution of $\chi^{2}$ for position matching between a stub in the CMP chambers and an extrapolated CTC track to the CMP; $\chi^{2}(C M P)$. The distributions for muon candidates in the $J / \psi \rightarrow \mu \mu$ events (solid) and for the jet sample (dotted) are shown. . . . . . . . . . . . .

4.9 The energy of electromagnetic tower which a muon traverses. The distributions from the $J / \psi \rightarrow \mu \mu$ (solid) and the jet sample (dotted) are shown. ........................... 80

4.10 The energy of hadronic tower which a muon traverses. The distributions from the $J / \psi \rightarrow \mu \mu$ (solid) and the jet sample (dotted) are shown. . . . 81

4.11 The energy sum of electromagnetic and hadronic tower which a muon traverses. The distributions from the $J / \psi \rightarrow \mu \mu$ (solid) and the jet sample

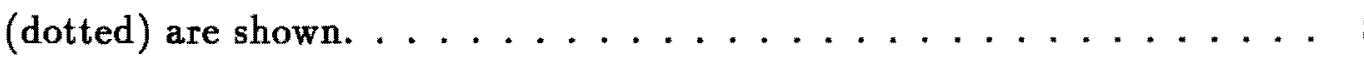


4.12 The HAD/EM distributions for central electrons in the $J / \psi \rightarrow e e$ (solid) and the jet sample (dotted) . . . . . . . . . . . 83

4.13 The E/P distributions for central electrons in the $J / \psi \rightarrow e e$ (solid) and the jet sample (dotted). . . . . . . . . . . . . . 84

4.14 The lateral shower shape, $\chi_{\text {strip }}^{2}$, distributions for electrons in the $J / \psi \rightarrow$ ee events (solid) and for EM clusters of the jet sample (dotted). . . . .

4.15 The position difference in $(r-\phi)$ plane between the position measured in the CES and that of a track extrapolated to the CES, $\triangle X(C E S)$. The distributions for electrons in the $J / \psi \rightarrow e e$ events (solid) and for EM clusters of the jet sample (dotted). . . . . . . . . . .

4.16 The position difference along the beam direction between the $Z$ position measured in the CES and that of a track extrapolated to the CES, $\triangle Z(C E S)$. The distributions for electrons in the $J / \psi \rightarrow$ ee events (solid) and for EM clusters in the jet sample (dotted). . . . . . . . . . 87

4.17 The lateral shower sharing, $L_{\mathrm{shr}}$, for electrons in the $J / \psi \rightarrow e e$ (solid) and for EM clusters in the jet sample (dotted). . . . . . . . .

4.18 The radial distance of the conversion points from the central conversion

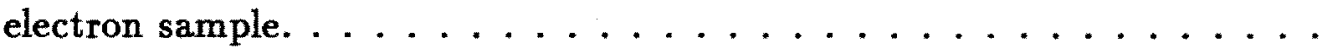

4.19 The $z$ position of event vertex for $J / \psi \rightarrow \mu \mu$ events. The measured distribution and the fitted gaussian function are shown. . . . . . . .

4.20 The missing transverse energy distributions for $J / \psi \rightarrow \mu \mu$ (solid) and

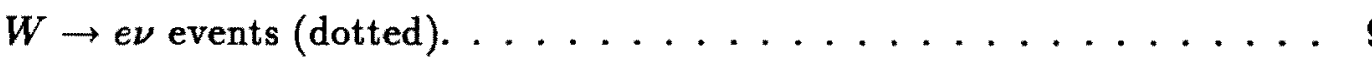

4.21 Jet axis finding algorithm. . . . . . . . . . . . . . . 92

4.22 The muon $P_{T}^{r e l}$ distributions for $b \bar{b}$ and $c \bar{c}$ Monte Carlo events. . . . . . 93

4.23 The electron $P_{T}^{r e l}$ distributions for $b \bar{b}$ and $c \bar{c}$ Monte Carlo events. . . . . . 94 
4.24 (a) Monte Carlo distribution of $\Delta \phi(e \mu)$ for semileptonic cascade decays. (b) Monte Carlo distribution of $\Delta \phi(e \mu)$ for $b \bar{b}$ direct production, $b \bar{b}$ production from gluon splitting and all (solid line). (c) The $\Delta \phi(e \mu)$ distribution for opposite-sign (solid line) and like-sign (dashed line) $e \mu$ events.

5.1 Invariant mass of a muon candidate track and an oppositely charged hadron track with a $\pi-\pi$ mass assignment. The dashed (solid) histogram is for the case where the muon candidate track passed the muon identifcation cut (the loose cut). . . . . . . . . . . . . . 127

5.2 Invariant mass of a muon candidate track and an oppositely charged hadron track with a $K$ - $K$ mass assignment. The dashed (solid) histogram is for the case where the muon candidate track passed the muon identification cut (the loose cut) . . . . . . . . . . . . . . . . . . . 128

5.3 Invariant mass distributions for dimuons. (a) One of the two muons passed the muon identification cut and the other muon passed the loose one. (b) Both of the two muons passed the muon identification cut. . . . 129

5.4 (a) $H A D / E M$ distribution for electron candidates with $\Delta X(C E S)>6$ $\mathrm{cm}$. Electron candidates in the hatched area pass the electron identification cut except $\triangle X(C E S)$ cut. (b) Probabilities for fake electrons (photon + charged hadron overlap) as a function of the lower cut value

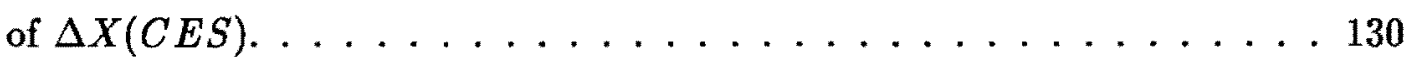

5.5 $H A D / E M$ distributions for generated pions passing the loose electron identification cut for (a) $P_{T}=5 \mathrm{GeV} / \mathrm{c}$ and (b) $P_{T}=7 \mathrm{GeV} / \mathrm{c}$. Pions in the hatched area pass the electron identification cut. . . . . . . 131

5.6 Invariant mass distributions for dielectrons. (a) One of the two electrons passed the electron identification selection and the other electron passed the loose one. (b) Both of the two electrons passed the electron identifcation selection. . . . . . . . . . . . . . . . . 132 
5.7 Transverse momentum distributions of electrons for conversion electron sample and the Monte Carlo data. The Monte Carlo data is normalized in a region of $1<P_{T}($ track $)<5 \mathrm{GeV} / \mathrm{c} . \ldots \ldots 133$

5.8 Charged particle multiplicities from the $B_{\varkappa}^{-}$meson. (a) Multiplicity of charged particles produced from $B_{u}^{-}$mesons. (b) Multiplicity of charged tracks (hadron or lepton) with $P_{T}>3 \mathrm{GeV} / \mathrm{c}$ and $|\eta|<0.6$. (c) Multiplicity of charged hadrons with $P_{T}>3 \mathrm{GeV} / \mathrm{c}$ and $|\eta|<0.6$. The leptons are removed in this distribution. The hatched area in multiplicity one bin in (b) and (c) represents negatively charged tracks and hadrons. . . . . . 134

5.9 Charged particle multiplicities from the $\bar{B}_{d}^{0}$ meson. (a) Multiplicity of charged particles produced from $\bar{B}_{d}^{0}$ mesons. (b) Multiplicity of charged tracks (hadron or lepton) with $P_{T}>3 \mathrm{GeV} / \mathrm{c}$ and $|\eta|<0.6$. (c) Multiplicity of charged hadrons with $P_{T}>3 \mathrm{GeV} / \mathrm{c}$ and $|\eta|<0.6$. The leptons are removed in this distribution. The hatched area in multiplicity one bin in (b) and (c) represents negatively charged tracks and hadrons. . . . . 135

5.10 Charged particle multiplicities from the $\bar{B}_{s}^{0}$ meson. (a) Multiplicity of charged particles produced from $\bar{B}_{s}^{0}$ mesons. (b) Multiplicity of charged tracks (hadron or lepton) with $P_{T}>3 \mathrm{GeV} / \mathrm{c}$ and $|\eta|<0.6$. (c) Multiplicity of charged hadrons with $P_{T}>3 \mathrm{GeV} / \mathrm{c}$ and $|\eta|<0.6$. The leptons are removed in this distribution. The hatched area in multiplicity one bin in (b) and (c) represents negatively charged tracks and hadrons. . . . . 136

5.11 Charged particle multiplicities from the $B_{u}^{+}$meson. (a) Multiplicity of charged particles produced from $B_{u}^{+}$mesons. (b) Multiplicity of charged tracks (hadron or lepton) with $P_{T}>3 \mathrm{GeV} / \mathrm{c}$ and $|\eta|<0.6$. (c) Multiplicity of charged hadrons with $P_{T}>3 \mathrm{GeV} / \mathrm{c}$ and $|\eta|<0.6$. The leptons are removed in this distribution. The hatched area in multiplicity one bin in (b) and (c) represents negatively charged tracks and hadrons. . . . . 137 
5.12 Charged particle multiplicities from the $B_{d}^{0}$ meson. (a) Multiplicity of charged particles produced from $B_{d}^{0}$ mesons. (b) Multiplicity of charged tracks (hadron or lepton) with $P_{T}>3 \mathrm{GeV} / \mathrm{c}$ and $|\eta|<0.6$. (c) Multiplicity of charged hadrons with $P_{T}>3 \mathrm{GeV} / \mathrm{c}$ and $|\eta|<0.6$. The leptons are removed in this distribution. The hatched area in multiplicity one bin in (b) and (c) represents negatively charged tracks and hadrons. . . . . 138

5.13 Charged particle multiplicities from the $B_{s}^{0}$ meson. (a) Multiplicity of charged particles produced from $B_{s}^{0}$ mesons. (b) Multiplicity of charged tracks (hadron or lepton) with $P_{T}>3 \mathrm{GeV} / \mathrm{c}$ and $|\eta|<0.6$. (c) Multiplicity of charged hadrons with $P_{T}>3 \mathrm{GeV} / \mathrm{c}$ and $|\eta|<0.6$. The leptons are removed in this distribution. The hatched area in multiplicity one bin in (b) and (c) represents negatively charged tracks and hadrons. . . . 139

5.14 The fraction of the sequential decays for different Peterson parameter values and structure functions. . . . . . . . . . . . . . . . . 140

5.15 The difference between the numbers of OS and LS events as a function of $P_{T}^{\text {rel }}$ (a) for muons and (b) for electrons. The points are observed data. The bashed line represents $b \bar{b}$ Monte Carlo data, the dotted line $c \bar{c}$ Monte Carlo data, the dot-dashed line fake lepton events, the solid line the sum of these three. The Monte Carlo data are generated using MRSD0-' structure function set. The contributions from $c \vec{c}$ and fake lepton events are not clear by visible for the electron case. . . . . . . . . . . . 14

5.16 The difference between the numbers of OS and LS events as a function of $P_{T}^{\text {rel }}$ (a) for muons and (b) for electrons. The points are observed data. The bashed line represents $b \bar{b}$ Monte Carlo data, the dotted line $c \bar{c}$ Monte Carlo data, the dot-dashed line fake lepton events, the solid line the sum of these three. The Monte Carlo data are generated using EHLQ structure function set. The contributions from $c \bar{c}$ and fake lepton events are not clear by visible for the electron case. . . . . . . . . . . . . 142 
5.17 The difference between the numbers of OS and LS events as a function of $P_{T}^{\text {rel }}$ (a) for muons and (b) for electrons. The points are observed data. The bashed line represents $b \bar{b}$ Monte Carlo data, the dotted line $c \bar{c}$ Monte Carlo data, the dot-dashed line fake lepton events, the solid line the sum of these three. The Monte Carlo data are generated using DFLM 260 structure function set. The contributions from $c \bar{c}$ and fake lepton events are not clear by visible for the electron case. . . . . . . . . . 143

5.18 The difference between the numbers of OS and LS events as a function of $P_{T}^{\text {rel }}$ (a) for muons and (b) for electrons. The points are observed data. The bashed line represents $b \bar{b}$ Monte Carlo data, the dotted line $c \bar{c}$ Monte Carlo data, the dot-dashed line fake lepton events, the solid line the sum of these three. The Monte Carlo data are generated using MRSD0-' structure function set. In this distributions, numbers of fake muons and electrons are normalized to $70+23$ and $29+77$, respectively. The contributions from $c \bar{c}$ and fake lepton events are not clear by visible for the electron case. . . . . . . . . . . . . . . . . . . . . 144

5.19 The difference between the numbers of OS and LS events as a function of $P_{T}^{\text {rel }}$ (a) for muons and (b) for electrons. The points are observed data. The bashed line represents $b \vec{b}$ Monte Carlo data, the dotted line $c \bar{c}$ Monte Carlo data, the dot-dashed line fake lepton events, the solid line the sum of these three. The Monte Carlo data are generated using MRSD0 -' structure function set. In this distributions, numbers of fake muons and electrons are normalized to $70-23$ and $29-77$, respectively. The contributions from $c \bar{c}$ and fake lepton events are not clear by visible

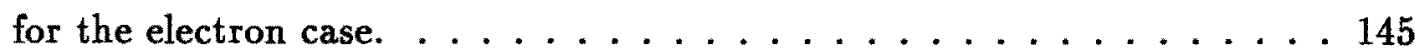

6.1 Comparison with other experiments. .............. 149 
6.2 The mixing parameter for $B_{d}^{0}$ versus that for $B_{s}^{0}$ assuming that $P_{d}$ and $P_{s}$ are 0.391 and 0.117 . The $\chi_{d}$ range is the ARGUS (1992\&1994) and CLEO (1993) combined result of $0.15 \pm 0.03$. The bands represent $\pm 1 \sigma$

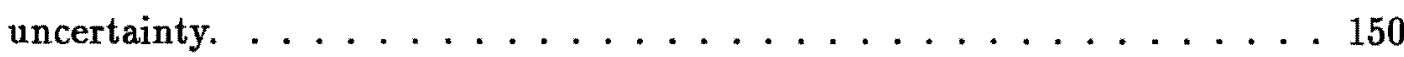

6.3 The mixing parameter for $B_{d}^{0}$ versus that for $B_{s}^{0}$ assuming $P_{d}$ and $P_{s}$ are 0.391 and 0.117 . The $\chi$ was calculated from our result and average value in the Particle Data Book. The $\chi_{d}$ is estimated by the ARGUS (1992\&1994) and CLEO (1993) combined result of $0.15 \pm 0.03$. The $68.3 \%$ (solid) and $95 \%$ (dashed) confidence areas are shown. . . . . . . . . 151

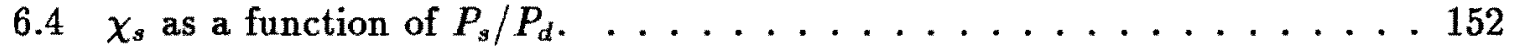

B.1 The probability distributions of $f_{s}$. The structure functions for the top, middle and bottom figures are MRSD0 -', EHLQ and DFLM, respectively. In each figure, the solid (dashed or dotted) line represents the probability with Peterson fragmentation parameter $\epsilon=0.003(0.006$ or 0.009$) \ldots \ldots 158$

B.2 The sum of probability distributions for $f_{s}$. The distribution is fitted by a Gaussian distribution. The fitted parameters of the Gaussian distribution are listed in the figure. . . . . . . . . . . . . 159 


\section{CDF Collaboration}

F. Abe, ${ }^{13}$ M. G. Albrow ${ }^{7}$ S. R. Amendolia, ${ }^{23}$ D. Amidei, ${ }^{16}$ J. Antos,${ }^{28}$ C. AnwayWiese, ${ }^{4}$ G. Apollinari, ${ }^{26}$ H. Areti,${ }^{7}$ M. Atac, ${ }^{7}$ P. Auchincloss,${ }^{25}$ F. Azfar,${ }^{21}$ P. Azzi,${ }^{20}$ N. Bacchetta, ${ }^{18}$ W. Badgett,${ }^{16}$ M. W. Bailey,${ }^{18}$ J. Bao,${ }^{35}$ P. de Barbaro, ${ }^{25}$ A. BarbaroGaltieri,${ }^{14}$ V. E. Barnes, ${ }^{24}$ B. A. Barnett,,${ }^{12}$ P. Bartalini, ${ }^{23}$ G. Bauer,${ }^{15}$ T. Baumann, ${ }^{9}$ F. Bedeschi, ${ }^{23}$ S. Behrends,${ }^{3}$ S. Belforte,${ }^{23}$ G. Bellettini, ${ }^{23}$ J. Bellinger,${ }^{34}$ D. Benjamin,${ }^{31}$

J. Benlloch, ${ }^{15}$ J. Bensinger,${ }^{3}$ D. Benton,${ }^{21}$ A. Beretvas, ${ }^{7}$ J. P. Berge,${ }^{7}$ S. Bertolucci ${ }^{8}$ A. Bhatti, ${ }^{26}$ K. Biery, ${ }^{11}$ M. Binkley, ${ }^{7}$ F. Bird, ${ }^{29}$ D. Bisello, ${ }^{20}$ R. E. Blair,${ }^{1}$ C. Blocker,${ }^{29}$ A. Bodek,${ }^{25}$ W. Bokhari, ${ }^{15}$ V. Bolognesi, ${ }^{23}$ D. Bortoletto,,${ }^{24}$ C. Boswell, ${ }^{12}$ T. Boulos, ${ }^{14}$ G. Brandenburg, ${ }^{9}$ C. Bromberg, ${ }^{17}$ E. Buckley-Geer ${ }^{7}$ H. S. Budd,${ }^{25}$ K. Burkett, ${ }^{16}$ G. Busetto, ${ }^{20}$ A. Byon-Wagner,${ }^{7}$ K. L. Byrum, ${ }^{1}$ J. Cammerata, ${ }^{12}$ C. Campagnari, ${ }^{7}$ M. Campbell,${ }^{16}$ A. Caner,${ }^{7}$ W. Carithers, ${ }^{14}$ D. Carlsmith,${ }^{34}$ A. Castro, ${ }^{20}$ Y. Cen, ${ }^{21}$ F. Cervelli, ${ }^{23}$ H. Y. Chao, ${ }^{28}$ J. Chapman, ${ }^{16}$ M.-T. Cheng, ${ }^{28}$ G. Chiarelli, ${ }^{8}$ T. Chikamatsu, ${ }^{32}$ C. N. Chiou, ${ }^{28}$ S. Cihangir,${ }^{7}$ A. G. Clark, ${ }^{23}$ M. Cobal, ${ }^{23}$ M. Contreras,${ }^{5}$ J. Conway,,${ }^{27}$ J. Cooper,${ }^{7}$ M. Cordelli, ${ }^{8}$ C. Couyoumtzelis, ${ }^{23}$ D. Crane,${ }^{1}$ J. D. Cunningham, ${ }^{3}$ T. Daniels, ${ }^{15}$ F. DeJongh, ${ }^{7}$ S. Delchamps, ${ }^{7}$ S. Dell'Agnello, ${ }^{23}$ M. Dell'Orso, ${ }^{23}$ L. Demortier, ${ }^{26}$ B. Denby, ${ }^{23}$ M. Deninno, ${ }^{2}$ P. F. Derwent, ${ }^{16}$ T. Devlin, ${ }^{27}$ M. Dickson, ${ }^{25}$ J. R. Dittmann, ${ }^{6}$ S. Donati, ${ }^{23}$ R. B. Drucker,${ }^{14}$ A. Dunn,${ }^{16}$ K. Einsweiler,${ }^{14}$ J. E. Elias, ${ }^{7}$ R. Ely, ${ }^{14}$ E. Engels, Jr. ${ }^{22}$ S. Eno, ${ }^{5}$ D. Errede,${ }^{10}$ S. Errede, ${ }^{10}$ Q. Fan,${ }^{25}$ B. Farhat,${ }^{15}$ I. Fiori, ${ }^{2}$ B. Flaugher,${ }^{7}$ G. W. Foster,${ }^{7}$ M. Franklin, ${ }^{9}$ M. Frautschi, ${ }^{18}$ J. Freeman, ${ }^{7}$ J. Friedman, ${ }^{15}$ H. Frisch, ${ }^{5}$ A. Fry ${ }^{29}$ T. A. Fuess, ${ }^{1}$ Y. Fukui, ${ }^{13}$ S. Funaki, ${ }^{32}$ G. Gagliardi, ${ }^{23}$ S. Galeotti, ${ }^{23}$ M. Gallinaro, ${ }^{20}$ A. F. Garfinkel,${ }^{24}$ S. Geer, ${ }^{7}$ D. W. Gerdes,${ }^{16}$ P. Giannetti, ${ }^{23}$ N. Giokaris,${ }^{26}$ P. Giromini, ${ }^{8}$ L. Gladney, ${ }^{21}$ D. Glenzinski, ${ }^{12}$ M. Gold ${ }^{18}$ J. Gonzalez, ${ }^{21}$ A. Gordon, ${ }^{9}$ A. T. Goshaw,${ }^{6} \mathrm{~K}$. Goulianos, ${ }^{26}$ H. Grassmann,${ }^{6}$ A. Grewal, ${ }^{21}$ G. Grieco, ${ }^{23}$ L. Groer,${ }^{27}$ C. Grosso-Pilcher, ${ }^{5}$ C. Haber, ${ }^{14}$ S. R. Hahn, ${ }^{7}$ R. Hamilton, ${ }^{9}$ R. Handler, ${ }^{34}$ R. M. Hans,${ }^{35}$ K. Hara,${ }^{32}$ B. Harral,${ }^{21}$ R. M. Harris, ${ }^{7}$ S. A. Hauger,${ }^{6}$ J. Hauser, ${ }^{4}$ C. Hawk, ${ }^{27}$ J. Heinrich, ${ }^{21}$ D. Cronin-Hennessy, ${ }^{6}$ R. Hollebeek, ${ }^{21}$ 
L. Holloway, ${ }^{10}$ A. Hölscher, ${ }^{11}$ S. Hong, ${ }^{16}$ G. Houk, ${ }^{21}$ P. Hu, ${ }^{22}$ B. T. Huffman, ${ }^{22}$ R. Hughes, ${ }^{25}$ P. Hurst, ${ }^{9}$ J. Huston, ${ }^{17}$ J. Huth, ${ }^{9}$ J. Hylen,${ }^{7}$ M. Incagli, ${ }^{23}$ J. Incandela, ${ }^{7}$ H. Iso, ${ }^{32}$ H. Jensen, ${ }^{7}$ C. P. Jessop, ${ }^{9}$ U. Joshi, ${ }^{7}$ R. W. Kadel, ${ }^{14}$ E. Kajfasz,${ }^{7 a}$ T. Kamon, ${ }^{30}$ T. Kaneko, ${ }^{32}$ D. A. Kardelis, ${ }^{10}$ H. Kasha, ${ }^{35}$ Y. Kato, ${ }^{19}$ L. Keeble,${ }^{8}$ R. D. Kennedy, ${ }^{27}$ R. Kephart, ${ }^{7}$ P. Kesten, ${ }^{14}$ D. Kestenbaum, ${ }^{9}$ R. M. Keup, ${ }^{10}$ H. Keutelian, ${ }^{7}$ F. Keyvan,${ }^{4}$ D. H. Kim, ${ }^{7}$ H. S. Kim, ${ }^{11}$ S. B. Kim, ${ }^{16}$ S. H. Kim, ${ }^{32}$ Y. K. Kim, ${ }^{14}$ L. Kirsch, ${ }^{3}$ P. Koehn, ${ }^{25}$ K. Kondo, ${ }^{32}$ J. Konigsberg, ${ }^{9}$ S. Kopp,${ }^{5}$ K. Kordas,${ }^{11}$ W. Koska,${ }^{7}$ E. Kovacs,${ }^{7 a}$ W. Kowald ${ }^{6}$ M. Krasberg, ${ }^{16}$ J. Kroll, ${ }^{7}$ M. Kruse, ${ }^{24}$ S. E. Kuhlmann, ${ }^{1}$ E. Kuns ${ }^{27}$ A. T. Laasanen, ${ }^{24}$ N. Labanca, ${ }^{23}$ S. Lammel, ${ }^{4}$ J. I. Lamoureux, ${ }^{3}$ T. LeCompte, ${ }^{10}$ S. Leone ${ }^{23}$ J. D. Lewis, ${ }^{7}$ P. Limon, ${ }^{7}$ M. Lindgren, ${ }^{4}$ T. M. Liss, ${ }^{10}$ N. Lockyer,${ }^{21}$ C. Loomis, ${ }^{27}$ O. Long, ${ }^{21}$ M. Loreti, ${ }^{20}$ E. H. Low,${ }^{21}$ J. Lu, ${ }^{30}$ D. Lucchesi, ${ }^{23}$ C. B. Luchini, ${ }^{10}$ P. Lukens, ${ }^{7}$ P. Maas, ${ }^{34}$ K. Maeshima, ${ }^{7}$ A. Maghakian, ${ }^{26}$ P. Maksimovic, ${ }^{15}$ M. Mangano, ${ }^{23}$ J. Mansour, ${ }^{17}$ M. Mariotti, ${ }^{23}$ J. P. Marriner, ${ }^{7}$ A. Martin, ${ }^{10}$ J. A. J. Matthews, ${ }^{18}$ R. Mattingly, ${ }^{15}$ P. McIntyre, ${ }^{30}$ P. Melese, ${ }^{26}$ A. Menzione, ${ }^{23}$ E. Meschi, ${ }^{23}$ G. Michail, ${ }^{9}$ S. Mikamo, ${ }^{13}$ M. Miller, ${ }^{5}$ R. Miller, ${ }^{17}$ T. Mimashi, ${ }^{32}$ S. Miscetti, ${ }^{8}$ M. Mishina, ${ }^{13}$ H. Mitsushio, ${ }^{32}$ S. Miyashita, ${ }^{32}$ Y. Morita, ${ }^{13}$ S. Moulding, ${ }^{26}$ J. Mueller,${ }^{27}$ A. Mukherjee,${ }^{7}$ T. Muller, ${ }^{4}$ P. Musgrave, ${ }^{11}$ L. F. Nakae, ${ }^{29}$ I. Nakano, ${ }^{32}$ C. Nelson, ${ }^{7}$ D. Neuberger,${ }^{4}$ C. Newman-Holmes, ${ }^{7}$ L. Nodulman, ${ }^{1}$ S. Ogawa, ${ }^{32}$ S. H. Oh, ${ }^{6}$ K. E. Ohl, ${ }^{35}$ R. Oishi, ${ }^{32}$ T. Okusawa, ${ }^{19}$ C. Pagliarone, ${ }^{23}$ R. Paoletti, ${ }^{23}$ V. Papadimitriou, ${ }^{31}$ S. Park, ${ }^{7}$ J. Patrick, ${ }^{7}$ G. Pauletta, ${ }^{23}$ M. Paulini, ${ }^{14}$ L. Pescara, ${ }^{20}$ M. D. Peters, ${ }^{14}$ T. J. Phillips, ${ }^{6}$ G. Piacentino, ${ }^{2}$ M. Pillai, ${ }^{25}$ R. Plunkett, ${ }^{7}$ L. Pondrom, ${ }^{34}$ N. Produit, ${ }^{14}$ J. Proudfoot, ${ }^{1}$ F. Ptohos, ${ }^{9}$ G. Punzi, ${ }^{23}$ K. Ragan, ${ }^{11}$ F. Rimondi, ${ }^{2}$ L. Ristori, ${ }^{23}$ M. Roach-Bellino, ${ }^{33}$ W. J. Robertson, ${ }^{6}$ T. Rodrigo, ${ }^{7}$ J. Romano, ${ }^{5}$ L. Rosenson, ${ }^{15}$ W. K. Sakumoto, ${ }^{25}$ D. Saltzberg, ${ }^{5}$ A. Sansoni, ${ }^{8}$ V. Scarpine, ${ }^{30}$ A. Schindler, ${ }^{14}$ P. Schlabach, ${ }^{9}$ E. E. Schmidt,${ }^{7}$ M. P. Schmidt, ${ }^{35}$ O. Schneider, ${ }^{14}$ G. F. Sciacca, ${ }^{23}$ A. Scribano, ${ }^{23}$ S. Segler,${ }^{7}$ S. Seidel, ${ }^{18}$ Y. Seiya, ${ }^{32}$ G. Sganos, ${ }^{11}$ A. Sgolacchia, ${ }^{2}$ M. Shapiro, ${ }^{14}$ N. M. Shaw ${ }^{24}$ Q. Shen, ${ }^{24}$ P. F. Shepard, ${ }^{22}$ M. Shimojima, ${ }^{32}$ M. Shochet, ${ }^{5}$ J. Siegrist, ${ }^{29}$ A. Sill, ${ }^{31}$ P. Sinervo, ${ }^{11}$ P. Singh, ${ }^{22}$ J. Skarha, ${ }^{12}$ K. Sliwa, ${ }^{33}$ D. A. Smith, ${ }^{23}$ F. D. Snider, ${ }^{12}$ L. Song, ${ }^{7}$ T. Song, ${ }^{16}$ J. Spalding, ${ }^{7}$ L. Spiegel, ${ }^{7}$ 
P. Sphicas, ${ }^{15}$ A. Spies, ${ }^{12}$ L. Stanco, ${ }^{20}$ J. Steele, ${ }^{34}$ A. Stefanini,${ }^{23}$ K. Strahl,${ }^{11}$ J. Strait,${ }^{7}$ D. Stuart, ${ }^{7}$ G. Sullivan, ${ }^{5}$ K. Sumorok, ${ }^{15}$ R. L. Swartz, Jr.,${ }^{10}$ T. Takahashi, ${ }^{19}$ K. Takikawa, ${ }^{32}$ F. Tartarelli, ${ }^{23}$ W. Taylor, ${ }^{11}$ P. K. Teng, ${ }^{28}$ Y. Teramoto,,${ }^{19}$ S. Tether,${ }^{15}$ D. Theriot,${ }^{7}$ J. Thomas, ${ }^{29}$ T. L. Thomas, ${ }^{18}$ R. Thun, ${ }^{16}$ M. Timko, ${ }^{33}$ P. Tipton, ${ }^{25}$ A. Titov,${ }^{26}$ S. Tkaczyk,${ }^{7}$ K. Tollefson,${ }^{25}$ A. Tollestrup,${ }^{7}$ J. Tonnison,${ }^{24}$ J. F. de Troconiz,${ }^{9}$ J. Tseng,${ }^{12}$ M. Turcotte,${ }^{29}$ N. Turini, ${ }^{2}$ N. Uemura,${ }^{32}$ F. Ukegawa, ${ }^{21}$ G. Unal, ${ }^{21}$ S. van den Brink, ${ }^{22}$ S. Vejcik, III,${ }^{16}$ R. Vidal,${ }^{7}$ M. Vondracek,${ }^{10}$ R. G. Wagner,${ }^{1}$ R. L. Wagner,${ }^{7}$ N. Wainer,${ }^{7}$ R. C. Walker, ${ }^{25}$ C. H. Wang, ${ }^{28}$ G. Wang, ${ }^{23}$ J. Wang, ${ }^{5}$ M. J. Wang, ${ }^{28}$ Q. F. Wang, ${ }^{26}$ A. Warburton, ${ }^{11}$ G. Watts, ${ }^{25}$ T. Watts, ${ }^{27}$ R. Webb, ${ }^{30}$ C. Wendt, ${ }^{34}$ H. Wenzel,,${ }^{14}$ W. C. Wester, III, ${ }^{14}$ T. Westhusing, ${ }^{10}$ A. B. Wicklund, ${ }^{1}$ E. Wicklund, ${ }^{7}$ R. Wilkinson, ${ }^{21}$ H. H. Williams, ${ }^{21}$ P. Wilson, ${ }^{5}$ B. L. Winer, ${ }^{25}$ J. Wolinski, ${ }^{30}$ D. Y. Wu,${ }^{16} \mathrm{X}$. Wu,${ }^{23}$ J. Wyss, ${ }^{20}$ A. Yagil,${ }^{7}$ W. Yao, ${ }^{14}$ K. Yasuoka,${ }^{32}$ Y. Ye,${ }^{11}$ G. P. Yeh,${ }^{7}$ P. Yeh,${ }^{28}$ M. Yin, ${ }^{6}$ J. Yoh, ${ }^{7}$ T. Yoshida, ${ }^{19}$ D. Yovanovitch, ${ }^{7}$ I. Yu, ${ }^{35}$ J. C. Yun, ${ }^{7}$ A. Zanetti, ${ }^{23}$ F. Zetti, ${ }^{23}$ L. Zhang, ${ }^{34}$ S. Zhang, ${ }^{16}$ W. Zhang, ${ }^{21}$ and S. Zucchelli ${ }^{2}$

1 Argonne National Laboratory, Argonne, Illinois 60439

2 Istituto Nazionale di Fisica Nucleare, University of Bologna, I-40126 Bologna, Italy

3 Brandeis University, Waltham, Massachusetts 02254

4 University of California at Los Angeles, Los Angeles, California 90024

5 University of Chicago, Chicago, Illinois 60637

6 Duke University, Durham, North Carolina 27708

7 Fermi National Accelerator Laboratory, Batavia, Illinois 60510

8 Laboratori Nazionali di Frascati, Istituto Nazionale di Fisica Nucleare, I-00044 Frascati, Italy

9 Harvard University, Cambridge, Massachusetts 02138

10 University of Illinois, Urbana, Illinois 61801

11 Institute of Particle Physics, McGill University, Montreal HSA 2T8, and University of Toronto,

Toronto M5S 1A7, Canada

12 The Johns Hopkins University, Baltimore, Maryland 21218

13 National Laboratory for High Energy Physics (KEK), Tsukuba, Ibaraki 905, Japan 
14 Lawrence Berkeley Laboratory, Berkeley, California 94720

15 Massachusetts Institute of Technology, Cambridge, Massachusetts 021\$9

16 University of Michigan, Ann Arbor, Michigan $\$ 8109$

17 Michigan State University, East Lansing, Michigan 48824

18 University of New Mexico, Albuquerque, New Mexico 87131

19 Osaka City University, Osaka 588, Japan

20 Universita di Padova, Instituto Nazionale di Fisica Nucleare, Sezione di Padova, I-\$5191 Padova, Italy

21 University of Pennsylvania, Philadelphia, Pennsyluania 19104

22 University of Pittsburgh, Pittsburgh, Pennsylvania 15260

23 Istituto Nazionale di Fisica Nucleare, University and Scuola Normale Superiore of Pisa, I-56100 Pisa, Italy

24 Purdue University, West Lafayette, Indiana 47907

25 University of Rochester, Rochester, New York 14627

26 Rockefeller University, New York, New York 10021

27 Rutgers University, Piscataway, New Jersey 08854

28 Academia Sinica, Taiwan 11589, Republic of China

29

Superconducting Super Collider Laboratory, Dallas, Texas 75237

30 Texas A $9 M$ University, College Station, Texas 77849

31 Texas Tech Uniyersity, Lubbock, Texas 79409

32 University of Tsukuba, Tsukuba, Ibaraki 905, Japan

33 Tufts University, Medford, Massachusetts 02155

34 University of Wisconsin, Madison, Wisconsin 53706

35 Yale University, New Haven, Connecticut 06511 


\section{Chapter 1}

\section{Introduction}

\subsection{Standard Model}

The Standard model is a model which can explain well all the observed phenomena at high energies in the present time. In the model, matter interacts with each other through three force mediators. The matter consists of quarks and leptons which are spin $1 / 2$ particles and are called fermions. The mediators are spin 1 particles and are called gauge bosons. Correspondingly to the gauge bosons, there are three types of interactions: the strong (gluon), electromagnetic (photon) and weak ( $W$ 's and $Z$ ) interactions. The standard model does not include gravitational interaction, but the observed phenomena are explained well without it because the effect of the gravitational interaction is negligibly small.

The quarks and leptons form sequential doublets as shown in Table 1.1. The common sequential structure of leptons and quarks is often called the family structure. The quarks and leptons of the three families are directly and/or indirectly confirmed experimentally. The top quark was observed by CDF and D0 experiments in last spring [1,2]. The mass of the top quark is measured to be $176 \pm 8 \pm 10 \mathrm{GeV} / \mathrm{c}^{2}$ by the CDF experiment.

The weak isospin eigenstates of quarks $\left(q^{\prime}\right)$ are not identical to the mass eigenstates (q). They are related by a unitary mixing matrix called Cabibbo-Kobayashi-Maskawa 


$$
\begin{array}{ll}
\text { Leptons } & \left(\begin{array}{c}
\nu_{e} \\
e^{-}
\end{array}\right)\left(\begin{array}{c}
\nu_{\mu} \\
\mu^{-}
\end{array}\right)\left(\begin{array}{l}
\nu_{\tau} \\
\tau^{-}
\end{array}\right) \\
\text {Quarks } & \left(\begin{array}{l}
u \\
d
\end{array}\right) \quad\left(\begin{array}{l}
c \\
s
\end{array}\right) \quad\left(\begin{array}{l}
t \\
b
\end{array}\right)
\end{array}
$$

Table 1.1: Leptons and quarks of the Standard Model

(CKM) matrix $[3]$ by

$$
\left(\begin{array}{c}
d^{\prime} \\
s^{\prime} \\
b^{\prime}
\end{array}\right)=\left(\begin{array}{ccc}
V_{u d} & V_{u s} & V_{u b} \\
V_{c d} & V_{c s} & V_{c b} \\
V_{t d} & V_{t s} & V_{t b}
\end{array}\right)\left(\begin{array}{l}
d \\
s \\
b
\end{array}\right)
$$

The values of the matrix elements are not calculable in the standard model but must be determined experimentally. The $90 \%$ confidence limits on the magnitude of the matrix elements are listed below [4].

$$
\left|V_{i j}\right|=\left(\begin{array}{llllll}
0.9747 & \text { to } 0.9759 & 0.218 & \text { to } 0.224 & 0.002 & \text { to } 0.005 \\
0.218 & \text { to } 0.224 & 0.9738 \text { to } 0.9752 & 0.032 & \text { to } 0.048 \\
0.004 & \text { to } 0.015 & 0.030 & \text { to } 0.048 & 0.9988 & \text { to } 0.9995
\end{array}\right)
$$

Due to the unitarity, the elements can be parameterized $[3,5]$ in terms of three angles and one phase:

$$
\left(\begin{array}{ccc}
c_{1} & -s_{1} c_{3} & -s_{1} s_{3} \\
s_{1} c_{2} & c_{1} c_{2} c_{3}-s_{2} s_{3} e^{i \delta} & c_{1} c_{2} s_{3}+s_{2} c_{3} e^{i \delta} \\
s_{1} s_{2} & c_{1} s_{2} c_{3}+c_{2} s_{3} e^{i \delta} & c_{1} s_{2} s_{3}-c_{2} c_{3} e^{i \delta}
\end{array}\right)
$$

where $c_{i}=\cos \theta_{i}, s_{i}=\sin \theta_{i}$ for $i=1,2,3$. 


\subsection{Basic formalism of mixing mechanism}

In this section we describe basic formulas of neutral $B$ meson mixing [6]. The mixing proceeds by a second order weak interaction as described by the "Box-diagrams" (Figure 1.1). The $B^{0} \bar{B}^{0}$ system is given by the phenomenological Hamiltonian matrix $H$ :

$$
H\left(\begin{array}{c}
B^{0} \\
\bar{B}^{0}
\end{array}\right)=\left(\begin{array}{cc}
M-i \Gamma / 2 & M_{12}-i \Gamma_{12} / 2 \\
M_{12}^{*}-i \Gamma_{12}^{*} / 2 & M-i \Gamma / 2
\end{array}\right)\left(\begin{array}{c}
B^{0} \\
\bar{B}^{0}
\end{array}\right) .
$$

The diagonal terms describe the decay of the neutral $B$ mesons with $M$ being the mass of the flavor eigenstates $B^{0}$ and $\bar{B}^{0}$ and $\Gamma$ their decay width. The off-diagonal terms are responsible for the $B^{0} \bar{B}^{0}$ transition. $M_{12}$ and $\Gamma_{12}$ can be determined from theory by evaluating the box-diagrams. $M_{12}$ corresponds to virtual $B^{0} \bar{B}^{0}$ transitions while $\Gamma_{12}$

describes real transitions due to decay modes which are common to the $B^{0}$ and $\bar{B}^{0}$, such as $B^{0}, \bar{B}^{0} \rightarrow \pi^{+} \pi^{-}$or $D^{+} D^{-}$. In contrast to the case of the $K^{0} \bar{K}^{0}$ system, these common decay modes are Cabibbo suppressed and therefore represent only a very small fraction of the total $B$ decay rate. The term $\Gamma_{12}$ in the $B^{0} \bar{B}^{0}$ system can therefore be neglected in practical calculation. Diagonalization of the Hamiltonian matrix leads to the CP eigenstates $B_{1}$ and $B_{2}$ which are linear combinations of the flavor eigenstates. Neglecting $\mathrm{CP}$ violation, we obtain:

$$
\begin{aligned}
& \left|B_{1}\right\rangle=\frac{1}{\sqrt{2}}\left(\left|B^{0}\right\rangle+\left|\bar{B}^{0}\right\rangle\right) \\
& \left|B_{2}\right\rangle=\frac{1}{\sqrt{2}}\left(\left|B^{0}\right\rangle-\left|\bar{B}^{0}\right\rangle\right)
\end{aligned}
$$

with masses $M_{1,2}$ and $\Gamma_{1,2}$ :

$$
\begin{gathered}
M_{1,2}=M \pm \frac{\Delta M}{2} \\
\Gamma_{1,2}=\Gamma \pm \frac{\Delta \Gamma}{2}
\end{gathered}
$$


The mass difference $\Delta M$ and the decay rate difference $\Delta \Gamma$ are given by:

$$
\Delta M=2 R e \sqrt{\left(M_{12}-i \Gamma_{12} / 2\right)\left(M_{12}^{*}-i \Gamma_{12}^{*} / 2\right)}
$$

and

$$
\Delta \Gamma=-4 I m \sqrt{\left(M_{12}-i \Gamma_{12} / 2\right)\left(M_{12}^{*}-i \Gamma_{12}^{*} / 2\right)} .
$$

The mass difference $\Delta M$ is a measure of the oscillation frequency to change from a $B^{0}$ to a $\bar{B}^{0}$ and vice versa. This is easily seen from the time evolution of one particle which at time $t=0$ is a $B^{0}$. The probability to find a $B^{0}\left(\bar{B}^{0}\right)$ at a time $t$ is given by $W_{B^{0}}(t)$ $\left(W_{\bar{B}^{0}}(t)\right)$ :

$$
\begin{aligned}
W_{B^{\circ}}(t) & =\frac{1}{4}\left[\exp \left(-\Gamma_{1} t\right)+\exp \left(-\Gamma_{2} t\right)+2 \exp (-\Gamma t) \cos \Delta M t\right] \\
W_{\bar{B}^{\circ}}(t) & =\frac{1}{4}\left[\exp \left(-\Gamma_{1} t\right)+\exp \left(-\Gamma_{2} t\right)-2 \exp (-\Gamma t) \cos \Delta M t\right]
\end{aligned}
$$

The measurements of these time dependences allow us to extract the values of $\Delta M, \Gamma_{1}$ and $\Gamma_{2}$. From time integrated rates $N\left(B^{0}\right)$ and $N\left(\bar{B}^{0}\right)$

$$
\begin{aligned}
& N\left(B^{0}\right)=\int_{0}^{\infty} W_{B^{0}}(t) d t=\frac{1}{4}\left[\frac{1}{\Gamma_{1}}+\frac{1}{\Gamma_{2}}+\frac{2 \Gamma}{\Gamma^{2}+(\Delta M)^{2}}\right] \\
& N\left(\bar{B}^{0}\right)=\int_{0}^{\infty} W_{\bar{B}^{0}}(t) d t=\frac{1}{4}\left[\frac{1}{\Gamma_{1}}+\frac{1}{\Gamma_{2}}-\frac{2 \Gamma}{\Gamma^{2}+(\Delta M)^{2}}\right]
\end{aligned}
$$

we can determine the parameter $r$ :

$$
r=\frac{N\left(\bar{B}^{0}\right)}{N\left(B^{0}\right)}=\frac{(\Delta M)^{2}+\left(\frac{\Delta \Gamma}{2}\right)^{2}}{2 \Gamma^{2}+(\Delta M)^{2}-\left(\frac{\Delta \Gamma}{2}\right)^{2}}
$$

With $\Delta \Gamma \approx 0$ as a consequence of $\Gamma_{12} \approx 0$ for $B^{0} \bar{B}^{0}$ system, the determination of $r$ gives a measurement of the ratio of the oscillation frequency and the decay width:

$$
r=\frac{x^{2}}{2+x^{2}}
$$


with

$$
x=\frac{\Delta M}{\Gamma}
$$

The $p \bar{p}$ colliding experiments can measure the ratio $r$ and the time dependent probabilities. The ratio is measured by using events in which both bottom quarks decay to leptons without knowing secondary vertex points. The time dependent probabilities are able to be measured by measuring the secondary vertex in events in which both bottom quarks decay to leptons. 

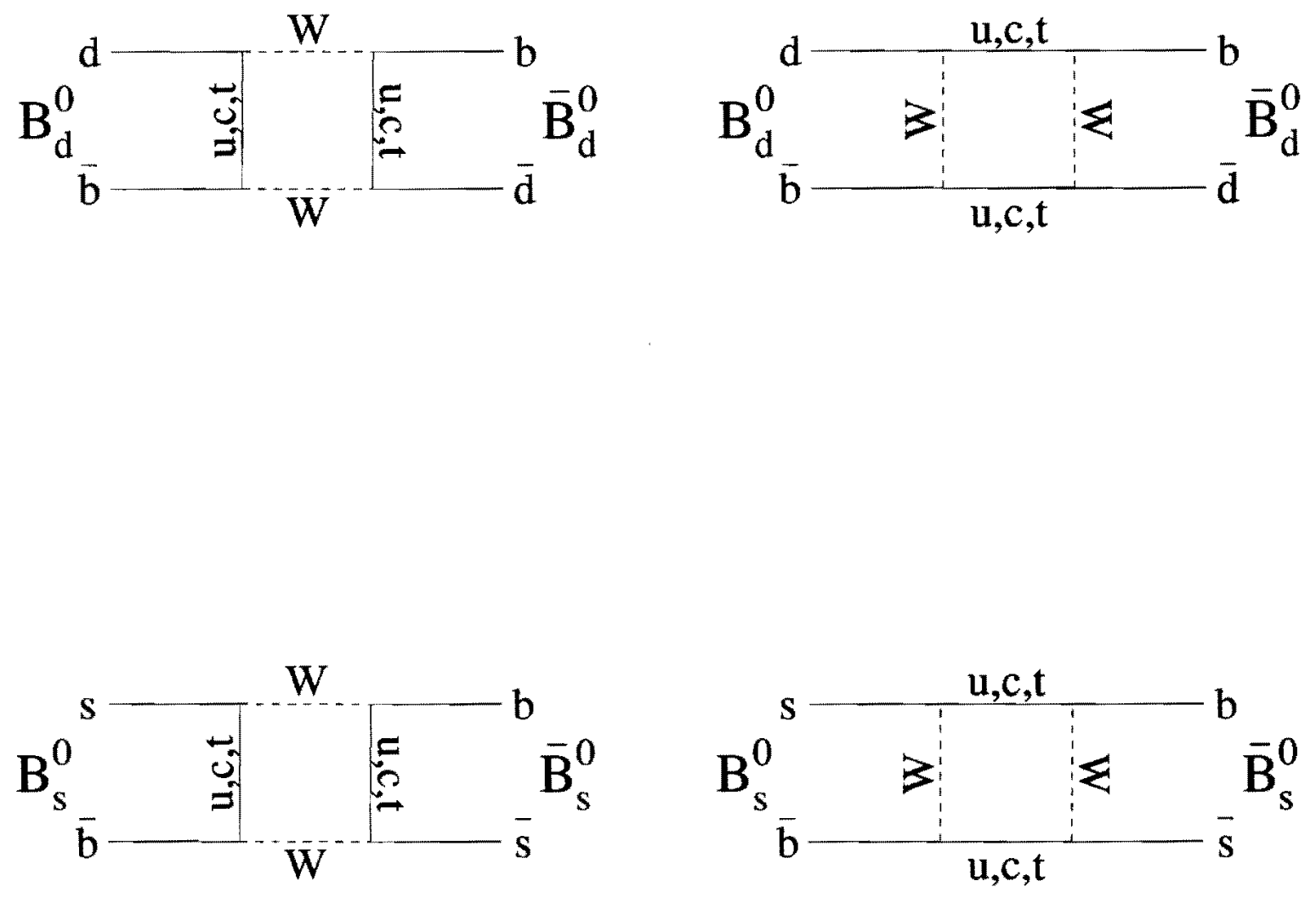

Figure 1.1: Feynman diagrams for the $B^{0} \bar{B}^{0}$ mixing process. 


\section{Chapter 2}

\section{Mixing in Neutral $B$ Mesons}

\section{$2.1 \quad B$ meson production}

\subsection{1 $b \bar{b}$ production}

In lowest order QCD, bottom quarks can be produced via gluon or a quark fusion mechanism, as shown in Fig. 2.1. Since the strong production process conserves flavor quantum number, $b$ and $\bar{b}$ quarks are always produced in pairs. The gluon fusion processes dominate at the Tevatron collider energy.

In the $c$ and $b$ quark productions, we have to take into consideration next-to-leading order diagrams, as shown in Fig. 2.2. The reason why higher order processes are as important as the lowest order ones is due to the large gluon-gluon scattering cross section. The process $g g \rightarrow g g$ has a cross section about 100 times that of $g g \rightarrow b \vec{b}$. Even though the $g \rightarrow b \bar{b}$ branching fraction is only $1 \%$, the $b \bar{b}$ production from the gluon splitting process is competitively as large as the lowest order contribution. 


\subsection{2 $b$ quark fragmentation}

When heavy quark $Q$ hadronizes, the heavy quark fragmentation function can be written as

$$
D_{Q}^{H}(z)=\frac{N_{H}}{z\left(1-\frac{1}{z}-\frac{\epsilon}{1-z}\right)^{2}}
$$

where $E^{H}$ and $E^{Q}$ are the energies of the hadron $H$ and the quark $Q, P_{\|}^{H}$ is the momentum component of the hadron $H$ parallel to the quark momentum $\left(P^{Q}\right)$ direction and $z=\left(E^{H}+P_{\|}^{H}\right) /\left(E^{Q}+P^{Q}\right)$. The $D_{Q}^{H}(z)$ is the probability that the heavy quark $Q$ fragments to hadron $H$ with value $z$, where $\epsilon$ is a constant proportional to $1 / M_{Q}^{2}$. The normalization constant $N_{H}$ is determined by

$$
\sum_{H} \int D_{Q}^{H}(z) d z=1
$$

where the summation is over all hadrons containing the heavy quark $Q$. This formula agrees well with experimental measurements for $b$ and $c$ quarks. The measured value of $\epsilon$ for $b$ quarks $[7]$ is

$$
\epsilon_{b}=0.006 \pm 0.001 \pm 0.002
$$

where the first is a statistical uncertainty and the second is systematic. The fragmentation function $D_{Q}^{H}(z)$ for $b$ and $c$ quarks are shown in Fig. 2.3. We can see that the bottom quark fragmentation is much harder than that of the charm quark. The hard fragmentation for $b$ quark clearly favors the detection of its daughter leptons at high $P_{T}$.

\section{$2.2 \quad B^{0} \quad \bar{B}^{0}$ mixing}

Especially in the case of $B_{q}$ mesons where $q$ stands for either an $s$ or $d$ quark, Eq. (1.17) is written by

$$
r_{q}=\frac{x_{q}^{2}}{2+x_{q}^{2}}
$$


Another parameter describing time-integrated mixing is $\chi_{q}$. This parameter describes the probability that a produced $B_{q}^{0}$ will have mixed into a $\bar{B}_{q}^{0}$ by the time it decays.

$$
\begin{aligned}
\chi_{q} & =\frac{N\left(\bar{B}^{0}\right)}{N\left(\bar{B}^{0}\right)+N\left(B^{0}\right)} \\
& =\frac{N\left(B_{q}^{0} \rightarrow \bar{B}_{q}^{0}\right)}{N\left(B_{q}^{0} \rightarrow \bar{B}_{q}^{0}\right)+N\left(B_{q}^{0} \rightarrow B_{q}^{0}\right)} \\
& =\frac{r_{q}}{1+r_{q}} \\
& =\frac{x_{q}^{2}}{2\left(1+x_{q}^{2}\right)}
\end{aligned}
$$

The possible range of these parameters are

$$
\begin{aligned}
& 0 \leq r_{q} \leq 1 \\
& 0 \leq \chi_{q} \leq \frac{1}{2}
\end{aligned}
$$

The time dependence of the $\left(B^{0} \rightarrow \bar{B}^{0}\right)$ probabilities is rewritten as

$$
\begin{aligned}
& W_{B^{0}}(t)=\frac{1}{4}\left[\exp \left(-\Gamma_{1} t\right)+\exp \left(-\Gamma_{2} t\right)+2 \exp (-\Gamma t) \cos \Delta M t\right] \\
& W_{\bar{B}^{0}}(t)=\frac{1}{4}\left[\exp \left(-\Gamma_{1} t\right)+\exp \left(-\Gamma_{2} t\right)-2 \exp (-\Gamma t) \cos \Delta M t\right]
\end{aligned}
$$

Figure 2.4 shows the probabilities of finding $B^{0}$ and $\bar{B}^{\circ}$ in an initially pure $B^{0}$ state for $B_{d}^{0}$ and $B_{s}^{0}$ mesons.

When $B$ meson mixing occurs by the second order $W$ exchange, as shown in Fig. 1.1, any of the quarks $t, c, u$ can participate in the box diagram but the $t$ quark is expected to be dominant due to its high mass. When the matrix elements are evaluated [8], one find

$$
x_{q} \equiv(\Delta M / \Gamma)_{B_{q}}=\frac{G_{F}^{2}}{6 \pi^{2}} m_{t}^{2} \tau_{B_{q}} B_{B_{q}} f_{B_{q}}^{2} M_{B_{q}}\left|V_{t q} V_{t b}^{*}\right|^{2} \frac{A\left(z_{t}\right)}{z_{t}} \eta_{Q C D}
$$

where $q$ stands for either an $s$ or $d$ quark, $G_{F}$ is the Fermi constant, $\tau_{B}$ is the $B^{0}$ lifetime and $\eta_{Q C D}$ is a correction from the mass scale dependence of the strong coupling constant. 
Taking $B_{d}^{0}$ as an example, we review the current knowledge of these parameters:

- $\tau_{B_{d}}$

$$
\tau_{B_{d}}=1.29 \pm 0.5 \mathrm{psec}[4]
$$

- $M_{B_{d}}$

$$
M_{B_{d}}=5.278 \mathrm{GeV}[4]
$$

- $A\left(z_{t}\right) / z_{t}$ is a slowly decreasing monotonic function varying from 1 at $m_{t o p}=0$ to about 0.6 at $m_{\text {top }}=150 \mathrm{GeV}$, where $z_{t}=m_{t}^{2} / m_{W}^{2}$.

- $\eta_{Q C D}$ is a QCD correction and is estimated to be about 0.85 .

- $B_{B} \cdot f_{B}^{2}$ describes the uncertainty about the hadronic matrix elements. The QCD coupling becomes large for low momentum transfer and thus perturbation theory is not applicable. This means that the value cannot be directly calculated. However, calculations using lattice QCD can estimate an approximate number. The standard value, combining the available theoretical and experimental information [8] is;

$$
B_{B_{d}} f_{B_{d}}^{2}=140 \pm 40 \mathrm{MeV}
$$

The $\left|V_{t d}\right|$ is estimated to be $\sim 0.004$ by putting the above values, the top mass measured by CDF and the $x_{d}$ measured by ARGUS and CLEO $[9,10]$.

There are many uncertainties in the calculation of $\chi_{q}$ but they all cancel when we look at the ratio of $B_{d}^{0}$ mixing and $B_{s}^{0}$ mixing. If we assume that the above five parameters are the same for $B_{d}^{0}$ and $B_{s}^{0}$ mesons, we find

$$
\beta \equiv \frac{x_{d}}{x_{s}}=\left|\frac{V_{t d}}{V_{t s}}\right|^{2}
$$

The only factor that remains is the ratio between the CKM matrix elements. From the 
unitarity of the CKM matrix, the ratio is estimated to be [8]

$$
\beta \leq 0.17
$$

If we use this parameter to make a relationship between $\chi_{d}$ and $\chi_{s}$ we find

$$
\chi_{d}<\beta^{2} \chi_{s} /\left[1-2 \chi_{s}\left(1-\beta^{2}\right)\right] \leq 0.0289 /\left(1-1.9422 \chi_{s}\right)
$$

Since $B_{d}^{0}$ and $B_{s}^{0}$ mesons are produced in $p \bar{p}$ collisions and since flavor tagging is made by looking at the charge of leptons, we define the mixing parameter, $\chi$, by

$$
\chi=\frac{N\left(b \rightarrow \bar{B}^{0} \rightarrow B^{0} \rightarrow l^{+}\right)}{N\left(b \rightarrow l^{ \pm}\right)}
$$

where $N\left(b \rightarrow l^{ \pm}\right)$is the number of $b$ quarks directly decaying to leptons and $N(b \rightarrow$ $\left.\bar{B}^{0} \rightarrow B^{0} \rightarrow l^{+}\right)$is the number of $b$ quarks directly decaying to leptons via $B^{0} \bar{B}^{0}$ mixing process. The denominator includes all possible hadrons formed with the $b$ quark, i.e, $\bar{B}_{d}^{0}, \bar{B}_{u}^{-}, \bar{B}_{s}^{0}$, etc. Since we do not distinguish between $B_{d}^{0}$ and $B_{s}^{0}$, the mixing parameter is written as

$$
\begin{aligned}
& \chi=\frac{N\left(b \rightarrow \bar{B}^{0} \rightarrow B^{0} \rightarrow l^{+}\right)}{N\left(b \rightarrow l^{ \pm}\right)} \\
& =\frac{N(b) \operatorname{prob}\left(b \rightarrow \bar{B}_{u}^{-}\right) \operatorname{prob}\left(\bar{B}_{u}^{-} \rightarrow B_{u}^{+}\right) \operatorname{Br}\left(B_{u}^{+} \rightarrow l^{+}\right)}{N(b) \operatorname{prob}\left(b \rightarrow \bar{B}_{u}^{-}\right)\left[\operatorname{prob}\left(\bar{B}_{u}^{-} \rightarrow B_{u}^{+}\right) \operatorname{Br}\left(B_{u}^{+} \rightarrow l^{+}\right)+\operatorname{prob}\left(\bar{B}_{u}^{-} \rightarrow \bar{B}_{u}^{-}\right) \operatorname{Br}\left(\bar{B}_{u}^{-} \rightarrow l^{-}\right)\right]} \\
& +N(b) \operatorname{prob}\left(b \rightarrow \bar{B}_{d}^{0}\right) \operatorname{prob}\left(\bar{B}_{d}^{0} \rightarrow B_{d}^{0}\right) B r\left(B_{d}^{0} \rightarrow l^{+}\right) \\
& \left.\overline{+N(b) \operatorname{prob}(b} \rightarrow \bar{B}_{d}^{0}\right)\left[\operatorname{prob}\left(\bar{B}_{d}^{0} \rightarrow B_{d}^{0}\right) B r\left(B_{d}^{0} \rightarrow \overline{l^{+}}\right)+\operatorname{prob}\left(\bar{B}_{d}^{0} \rightarrow \bar{B}_{d}^{0}\right) B r\left(\overline{B_{d}^{0} \rightarrow l^{-}}\right)\right] \\
& \frac{+N(b) \operatorname{prob}\left(b \rightarrow \bar{B}_{s}^{0}\right) \operatorname{prob}\left(\bar{B}_{s}^{0} \rightarrow B_{s}^{0}\right) B r\left(B_{s}^{0} \rightarrow l^{+}\right)}{+N(b) \operatorname{prob}\left(b \rightarrow \bar{B}_{s}^{0}\right)\left[\operatorname{prob}\left(\bar{B}_{s}^{0} \rightarrow B_{s}^{0}\right) B r\left(B_{s}^{0} \rightarrow l^{+}\right)+\operatorname{prob}\left(\bar{B}_{s}^{0} \rightarrow \bar{B}_{s}^{0}\right) B r\left(\bar{B}_{s}^{0} \rightarrow l^{-}\right)\right]} \\
& \frac{+N(b) \text { prob }\left(b \rightarrow \text { Baryon }_{b}\right) \text { prob }\left(\text { Baryon }_{b} \rightarrow \text { Baryon }_{\bar{b}}\right) \text { Br }\left(\text { Baryon }_{\vec{b}} \rightarrow l^{+}\right)}{+N\left(\text { b)prob }\left(b \rightarrow \text { Baryon }_{b}\right)\left[\text { prob }_{\left(\text {Baryon }_{b}\right.} \rightarrow \text { Baryon }_{\bar{b}}\right) \text { Br }\left(\text { Baryon }_{\bar{b}} \rightarrow l^{+}\right)\right.} \\
& {\overline{+p r o b}\left(\text { Baryon }_{b} \rightarrow \text { Baryon }_{b}\right) B r\left(\text { Baryon }_{b} \rightarrow l^{-}\right)}^{\text {. }}
\end{aligned}
$$

where $N(b)$ is the number of $b$ quarks, $\operatorname{prob}\left(b \rightarrow \bar{B}_{q}\right)$ is the production probability for 
$\bar{B}_{q}, \operatorname{prob}\left(\bar{B}_{q} \rightarrow B_{q}\right)$ is the probability that a produced $\vec{B}_{q}$ will have mixed into a $B_{q}$ and $\operatorname{Br}\left(B_{q} \rightarrow l\right)$ is a branching ratio for $B_{q} \rightarrow l$ decays. Since

$$
\begin{gathered}
\operatorname{prob}\left(\bar{B}_{u}^{-} \rightarrow B_{u}^{+}\right)=\operatorname{prob}\left(\text { Baryon }_{b} \rightarrow \text { Baryon }_{\bar{b}}\right)=0, \\
\operatorname{prob}\left(\bar{B}_{d}^{0} \rightarrow B_{d}^{0}\right)=\chi_{d}, \\
\operatorname{prob}\left(\bar{B}_{s}^{0} \rightarrow B_{s}^{0}\right)=\chi_{s}
\end{gathered}
$$

and

$$
\operatorname{prob}\left(\ddot{B}_{q} \rightarrow B_{q}\right)+\operatorname{prob}\left(\bar{B}_{q} \rightarrow \ddot{B}_{q}\right)=1 \quad \text { for } b \text { hadrons }
$$

we obtain

$$
\begin{aligned}
& \chi=\frac{N(b) p r o b\left(b \rightarrow \bar{B}_{d}^{0}\right) \chi_{d} B r\left(B_{d}^{0} \rightarrow l^{+}\right)+N(b) \operatorname{prob}\left(b \rightarrow \bar{B}_{s}^{0}\right) \chi_{s} B r\left(B_{s}^{0} \rightarrow l^{+}\right)}{N(b) B r\left(b \rightarrow l^{ \pm}\right)} \\
& =\frac{N(b) \operatorname{prob}\left(b \rightarrow \bar{B}_{d}^{0}\right) \chi_{d} B r\left(B_{d}^{0} \rightarrow l^{+}\right)}{N(b) B r\left(b \rightarrow l^{ \pm}\right)}+\frac{N(b) \operatorname{prob}\left(b \rightarrow \bar{B}_{s}^{0}\right) \chi_{s} B r\left(B_{s}^{0} \rightarrow l^{+}\right)}{N(b) B r\left(b \rightarrow l^{ \pm}\right)} \\
& =\operatorname{prob}\left(b \rightarrow \bar{B}_{d}^{0}\right) \frac{\operatorname{Br}\left(B_{d}^{0} \rightarrow l^{+}\right)}{B r\left(b \rightarrow l^{ \pm}\right)} \chi_{d}+\operatorname{prob}\left(b \rightarrow \bar{B}_{s}^{0}\right) \frac{B r\left(B_{s}^{0} \rightarrow l^{+}\right)}{B r\left(b \rightarrow l^{ \pm}\right)} \chi_{s} \\
& =P_{d} \chi_{d}+P_{s} \chi_{s}
\end{aligned}
$$

where

$$
\begin{aligned}
& \operatorname{Br}\left(b \rightarrow l^{ \pm}\right)= \\
& \operatorname{prob}\left(b \rightarrow \bar{B}_{u}^{-}\right) \operatorname{Br}\left(\bar{B}_{u}^{-} \rightarrow l^{-}\right)+ \\
& \operatorname{prob}\left(b \rightarrow \bar{B}_{d}^{0}\right)\left[\operatorname{prob}\left(\bar{B}_{d}^{0} \rightarrow B_{d}^{0}\right) B r\left(B_{d}^{0} \rightarrow l^{+}\right)+\operatorname{prob}\left(\bar{B}_{d}^{0} \rightarrow \bar{B}_{d}^{0}\right) B r\left(\bar{B}_{d}^{0} \rightarrow l^{-}\right)\right]+ \\
& \operatorname{prob}\left(b \rightarrow \bar{B}_{s}^{0}\right)\left[\operatorname{prob}\left(\bar{B}_{s}^{0} \rightarrow B_{s}^{0}\right) B r\left(B_{s}^{0} \rightarrow l^{+}\right)+\operatorname{prob}\left(\bar{B}_{s}^{0} \rightarrow \bar{B}_{s}^{0}\right) B r\left(\bar{B}_{s}^{0} \rightarrow l^{-}\right)\right]+ \\
& \operatorname{prob}\left(b \rightarrow \operatorname{Baryon} n_{b}\right) \operatorname{Br}\left(\text { Baryon }_{b} \rightarrow l^{-}\right), \\
& \chi_{d(s)} \equiv \frac{N\left(\bar{B}_{d(s)}^{0} \rightarrow B_{d(s)}^{0}\right)}{N\left(\bar{B}_{d(s)}^{0} \rightarrow \bar{B}_{d(s)}^{0}\right)+N\left(\bar{B}_{d(s)}^{0} \rightarrow B_{d(s)}^{0}\right)}
\end{aligned}
$$




$$
=\frac{N\left(B_{d(s)}^{0} \rightarrow \bar{B}_{d(s)}^{0}\right)}{N\left(\bar{B}_{d(s)}^{0} \rightarrow B_{d(s)}^{0}\right)+N\left(B_{d(s)}^{0} \rightarrow \bar{B}_{d(s)}^{0}\right)}
$$

and

$$
P_{d(s)}=\operatorname{prob}\left(b \rightarrow \bar{B}_{d(s)}^{0}\right) \frac{B r\left(B_{d(s)}^{0} \rightarrow l^{+} X\right)}{B r\left(b \rightarrow l^{ \pm} X\right)} .
$$

When a pair of $b \vec{b}$ quarks is generated and both directly decay to leptons $(b \rightarrow l+X)$, there are three different cases: (1) neither $b$ nor $\bar{b}$ quark undergoes mixing, (2) either $b$ or $\bar{b}$ quark undergoes mixing, and (3) both $b$ and $\bar{b}$ quarks undergo mixing. The fraction of case (1), (2) and (3) are $(1-\chi)^{2}, 2 \chi(1-\chi)$ and $\chi^{2}$, respectively. Taking into account the fact that the lepton charge flipps if the mixing occurs, one can easily find that a ratio of the number of like-sign (LS) dilepton event to that of opposite-sign (OS) dilepton events is expressed by the mixing parameter $\chi$ as

$$
\frac{\text { the number of LS dilepton events }}{\text { the number of OS dilepton events }}=\frac{2 \chi(1-\chi)}{\chi^{2}+(1-\chi)^{2}} \text {. }
$$

Therefore, the mixing parameter can be derived by measuring the ratio of LS to OS dilepton events.

The mixing parameter, $\chi$, can be estimated from Eq. (2.35) in principle, but some complications arise in actual analysis, due to sequential decay processes and background contributions.

\subsubsection{Previous measurements of mixing in the $B_{d}^{0}$ and $B_{s}^{0}$ sys- tem}

\section{Observation of $B_{d}^{0} \bar{B}_{d}^{0}$ mixing at $\Upsilon(4 S)[6]$}

The study of the $B_{d}^{0} \bar{B}_{d}^{0}$ mixing phenomenon is particularly simple at the $\Upsilon(4 S)$ which decays about a half of the time into a $B^{0} \bar{B}^{0}$ pair.

Since no other particles are produced besides this pair, the $\Upsilon(4 S)$ is a clean source of $B_{d}^{0}$ mesons which can be reconstructed or efficiently tagged. Background from $e^{+} e^{-}$ 
continuum contributions under the $\Upsilon(4 S)$ can be studied experimentally at the center-ofmass energies below the $\Upsilon(4 S)$ mass. The investigation of the $B_{d} \bar{B}_{d}$ mixing phenomenon on the $\Upsilon(4 S)$ has led to its observation by ARGUS and CLEO.

On the $\Upsilon(4 S)$ the straightforward strategy to study $B \bar{B}$ mixing is to reconstruct completely the two neutral $B_{d}$ mesons in the $\Upsilon(4 S)$ decay. This, however, is not trivial because of the small reconstruction efficiency. Nevertheless, the approach of fully reconstructing $\Upsilon(4 S)$ decays into $B_{d}^{0} B_{d}^{0}$ or $\bar{B}_{d}^{0} \bar{B}_{d}^{0}$ pairs led to the discovery of $B_{d} \bar{B}_{d}$ mixing. ARGUS succeeded in reconstructing a decay $\Upsilon(4 S) \rightarrow B_{d}^{0} B_{d}^{0}$ completely, where both $B_{d}^{0}$ mesons decayed into $D^{*-l^{+}} \nu$ with the following decay chains:

$$
\begin{aligned}
& B^{0} \rightarrow \quad D_{1}^{*-} \quad \mu_{1}^{+} \quad \nu_{1} \quad \text { and } \quad B^{0} \rightarrow \quad D_{2}^{*-} \quad \mu_{2}^{+} \quad \nu_{2} \\
& \hookrightarrow \quad \bar{D}^{0} \pi_{1 s}^{-} \quad \hookrightarrow \quad D^{-} \pi^{0} \\
& \hookrightarrow K_{1}^{+} \pi_{1}^{-} \quad \hookrightarrow \quad K_{2}^{+} \pi_{2}^{-} \pi_{2}^{-}
\end{aligned}
$$

In this simple event (Fig. 2.5) all particles were well identified and the masses of the intermediate states agreed well with the particle data table values. Kinematic considerations showed that the event is complete. The background for this event was considered to be completely negligible.

This one event demonstrated that the phenomenon of $B_{d}^{0} \bar{B}_{d}^{0}$ mixing must exist. However, a determination of the $B_{d}^{0} \bar{B}_{d}^{0}$ mixing strength needed other methods which provide much better statistics. One of the most efficient methods is the flavor tagging of $B$ mesons through their semileptonic decays. A $B$ meson will decay into an $e^{+}$or $\mu^{+}$plus a neutrino and hadrons with a branching ratio of about $10 \%$. Likewise a $\bar{B}_{d}$ meson will yield an $e^{-}$or $\mu^{-}$in its semileptonic decays. A search for mixing can thus be made by looking for events of: $\Upsilon(4 S) \rightarrow l^{ \pm} l^{ \pm}+X$. Besides the tagging of $B$ mesons through leptons, other particles which originate frequently in the weak decay of the $b$ quark can be used to tag the $b$ flavour of the decaying $B$ meson. For example, $D^{*+}, \Lambda_{c}$ and $D_{s}^{-}$ from $b$ quark decay products can be used as the tagging particles since they can be fully reconstructed.

ARGUS and CLEO have investigated events containing lepton pairs originating from 
$\Upsilon(4 S)$ decays. Due to the fact that the $B_{d}$ mesons were nearly at rest, two leptons from different mesons were uncorrelated. The dilepton events still included backgrounds from $J / \psi$ decays.

$B^{0} \bar{B}^{0}$ mixing is studied by tagging the flavor of the other $B^{0}$ mesons by a fast lepton. The $B^{0}$ mesons were most efficiently reconstructed in the channel $B^{0} \rightarrow D^{*-} l^{+} \nu$ where the undetectable neutrino was inferred from the missing mass squared against the $D^{*-} l^{+}$system, $M M^{2}$, which had to be consistent with zero for $B^{0}$ mesons originating from $\Upsilon(4 S)$ decays. The quantity $M M^{2}$ was given by the beam energy and the known energies and momenta of the $D^{*-}$ mesons and the $l^{+}$lepton:

$$
M M^{2}=\left(E_{\text {beam }}-\left(E_{D^{*-}}+E_{l^{+}}\right)\right)^{2}-\left(\vec{p}_{D^{*-}}+\vec{p}_{l^{+}}\right)^{2}
$$

As signature for the decay $B^{0} \rightarrow D^{*-} l^{+} \nu$ they obtained a prominent peak at $M M^{2}=0$. The momenta of $D^{*-}$ meson can be estimated by the pion momentum, in the decay $D^{*-} \rightarrow \bar{D}^{0} \pi^{-}$. When $B^{0} \rightarrow D^{*-} l^{+} \nu \rightarrow \bar{D}^{0} \pi^{-} l^{+} \nu$, right-sign combination of lepton and pion is $l^{+} \pi^{-}$.

The $M M^{2}$ spectra for events with $\left(l^{-} \pi^{+}\right) l^{-}$and $\left(l^{-} \pi^{+}\right) l^{+}$are shown in Fig. 2.6 and the momentum spectra of the additional leptons are shown in Fig. 2.7. The mixing parameter $\chi$ is given by:

$$
\chi_{d}=\frac{N\left(\bar{B}_{d}^{0} l^{-}\right)+N\left(B_{d}^{0} l^{+}\right)}{\left(N\left(\bar{B}_{d}^{0} l^{-}\right)+N\left(\bar{B}_{d}^{0} l^{+}\right)\right)+\left(N\left(\bar{B}_{d}^{0} l^{+}\right)+N\left(B_{d}^{0} l^{-}\right)\right)}
$$

where $N\left(\bar{B}_{d}^{0} l^{-}\right)+N\left(B_{d}^{0} l^{+}\right)$are given by the contents in the peak at $M M^{2}=0$ in Fig. 2.6 a) and $N\left(\bar{B}_{d}^{0} l^{+}\right)+N\left(B_{d}^{0} l^{-}\right)$by the corresponding one in Fig. $\left.2.6 \mathrm{~b}\right)$.

From these measurements, they estimated $\chi_{d}=0.162 \pm 0.043 \pm 0.039$ (ARGUS) [9] and $\chi_{d}=0.149 \pm 0.023 \pm 0.021$ (CLEO) [10]. 

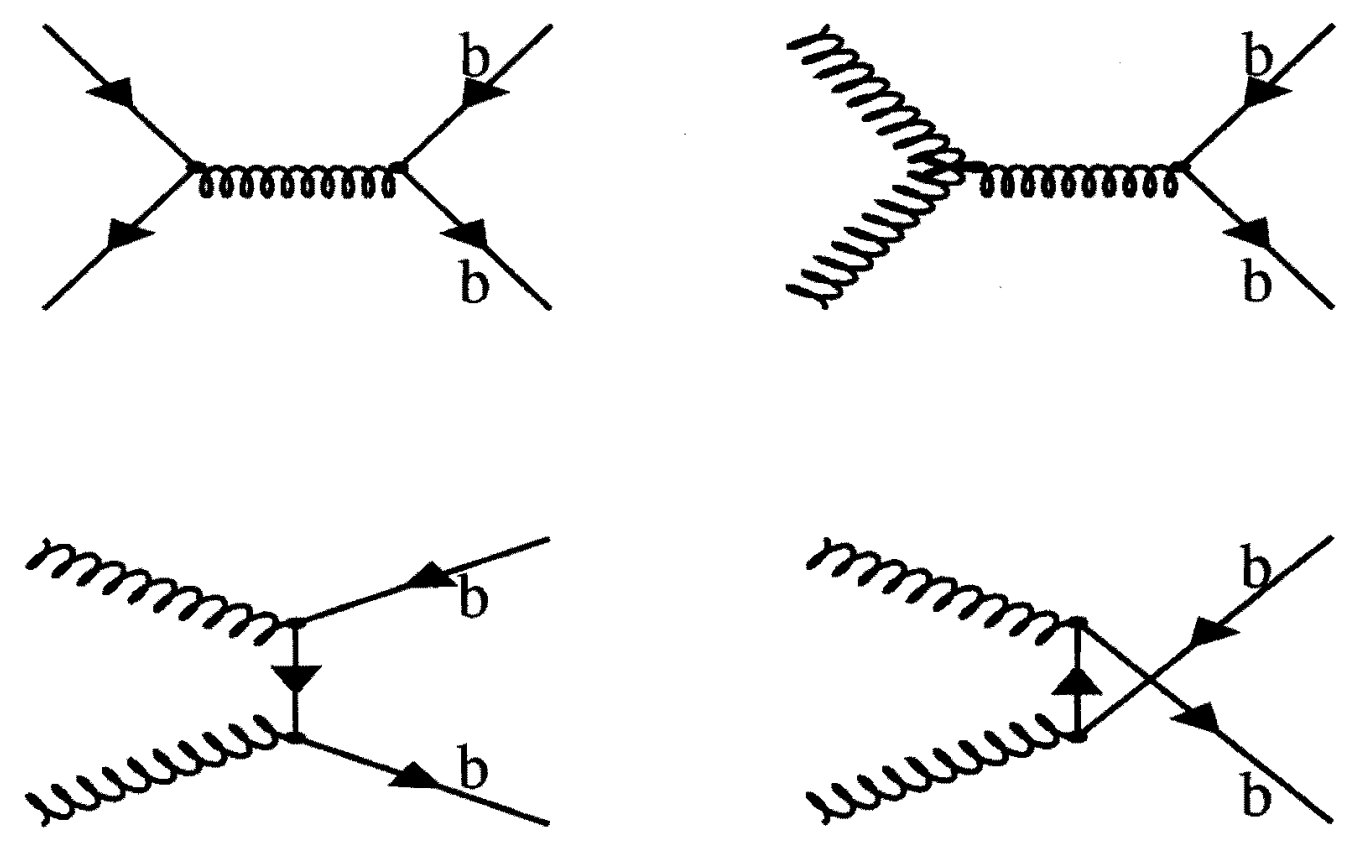

Figure 2.1: Feynman diagrams for lowest order $b \bar{b}$ production 
$\Upsilon(4 S)$ decays. Due to the fact that the $B_{d}$ mesons were nearly at rest, two leptons from different mesons were uncorrelated. The dilepton events still included backgrounds from $J / \psi$ decays.

$B^{0} \bar{B}^{0}$ mixing is studied by tagging the flavor of the other $B^{0}$ mesons by a fast lepton. The $B^{0}$ mesons were most efficiently reconstructed in the channel $B^{0} \rightarrow D^{*-} l^{+} \nu$ where the undetectable neutrino was inferred from the missing mass squared against the $D^{*-} l^{+}$system, $M M^{2}$, which had to be consistent with zero for $B^{0}$ mesons originating from $\Upsilon(4 S)$ decays. The quantity $M M^{2}$ was given by the beam energy and the known energies and momenta of the $D^{*-}$ mesons and the $l^{+}$lepton:

$$
M M^{2}=\left(E_{b e a m}-\left(E_{D^{*-}}+E_{l^{+}}\right)\right)^{2}-\left(\vec{p}_{D^{*-}}+\vec{p}_{l^{+}}\right)^{2}
$$

As signature for the decay $B^{0} \rightarrow D^{*-} l^{+} \nu$ they obtained a prominent peak at $M M^{2}=0$. The momenta of $D^{*-}$ meson can be estimated by the pion momentum, in the decay $D^{*-} \rightarrow \bar{D}^{0} \pi^{-}$. When $B^{0} \rightarrow D^{*-} l^{+} \nu \rightarrow \bar{D}^{0} \pi^{-} l^{+} \nu$, right-sign combination of lepton and pion is $l^{+} \pi^{-}$.

The $M M^{2}$ spectra for events with $\left(l^{-} \pi^{+}\right) l^{-}$and $\left(l^{-} \pi^{+}\right) l^{+}$are shown in Fig. 2.6 and the momentum spectra of the additional leptons are shown in Fig. 2.7. The mixing parameter $\chi$ is given by:

$$
\chi_{d}=\frac{N\left(\bar{B}_{d}^{0} l^{-}\right)+N\left(B_{d}^{0} l^{+}\right)}{\left(N\left(\bar{B}_{d}^{0} l^{-}\right)+N\left(B_{d}^{0} l^{+}\right)\right)+\left(N\left(\bar{B}_{d}^{0} l^{+}\right)+N\left(B_{d}^{0} l^{-}\right)\right)}
$$

where $N\left(\bar{B}_{d}^{0} l^{-}\right)+N\left(B_{d}^{0} l^{+}\right)$are given by the contents in the peak at $M M^{2}=0$ in Fig. 2.6 a) and $N\left(\bar{B}_{d}^{0} l^{+}\right)+N\left(B_{d}^{0} l^{-}\right)$by the corresponding one in Fig. $\left.2.6 \mathrm{~b}\right)$.

From these measurements, they estimated $\chi_{d}=0.162 \pm 0.043 \pm 0.039$ (ARGUS) [9] and $\chi_{d}=0.149 \pm 0.023 \pm 0.021$ (CLEO) [10]. 


\section{Observation of $B \bar{B}$ mixing on $Z^{0}$ and in $p \tilde{p}$ colliders [6].}

In $p \bar{p}$ colliders, the $B \bar{B}$ mixing were observed with the like-sign dimuon events by UA1. At LEP, the $B \bar{B}$ mixing has been studied using the $Z^{0} \rightarrow b \bar{b}$ process. In this case $b$ quarks fragment into a mixture of $B$ hadrons containing $B_{d}^{0}$ and $B_{s}^{0}$ mesons for which mixing can occur, which is a different point from the $B_{d} \bar{B}_{d}$ mixing at the $\Upsilon(4 S)$.

The fractions $P_{d}$ and $P_{s}$ of these incoherently produced $B_{d}^{0}$ and $B_{s}^{0}$ mesons are estimated from measurements of $B_{s}^{0}$ and $\Lambda_{b}^{0}$ production in $Z^{0}$ decays and were found to be: $P_{s}=0.152 \pm 0.042$ and $P_{\text {baryon }}=0.146 \pm 0.033[6]$. Assuming $P_{d}=P_{u}$, we gets $P_{d}=0.351 \pm 0.027$.

In time integrated measurements the $B$ mesons are tagged by their semileptonic decays and experimental signature for $B_{d}^{0} \bar{B}_{d}^{0}$ or $B_{s}^{0} \bar{B}_{s}^{0}$ mixing is given by an excess of like sign lepton pairs over the predicted number of like sign lepton pairs from background processes.

The leptons which are used to tag the $b$ flavor are again subject to backgrounds from other lepton sources like charm decays, converted photons or hadrons faking leptons. These backgrounds can be suppressed by requiring large momenta and large transverse momenta $p_{T}$ for both leptons with respect to the jet axis. The cut on large $p_{T}$ exploits the heaviness of the $B$ mesons compared to charm particles.

The amount of $B_{d}^{0} \bar{B}_{d}^{0}$ and $B_{s}^{0} \bar{B}_{s}^{0}$ mixing is determined from the like-sign and oppositesign lepton pairs by

$$
\frac{N\left(l^{ \pm} l^{ \pm}\right)}{N\left(l^{+} l^{-}\right)+N\left(l^{ \pm} l^{ \pm}\right)}=\frac{2 \chi(1-\chi) N_{o}+\left[(1-\chi)^{2}+\chi^{2}\right] N_{l}}{N_{o}+N_{l}}
$$

where $N_{o}\left(N_{l}\right)$ is the predicted opposite-sign (like-sign) dilepton rate in the case of no mixing. $N_{o}$ is given by the number of primary lepton pairs originating from $b \rightarrow l^{-} X$, $\bar{b} \rightarrow l^{+} X$ decays, while $N_{l}$ is given by the like-sign lepton pairs from $b \rightarrow l^{-} X, \bar{b} \rightarrow \bar{c} X$, $\bar{c} \rightarrow \boldsymbol{l}^{-} X$ decays.

The ALEPH and DELPHI used the jet charge technique for tagging $b$ flavor. The 


\begin{tabular}{l|l|l}
\hline \hline Experiment & Year & Value of mixing parameter $(\chi)$ \\
\hline ALEPH $[11]$ & 1994 & $0.114 \pm 0.014 \pm 0.008$ \\
L3 $[12]$ & 1994 & $0.123 \pm 0.012 \pm 0.008$ \\
D0 $[13]$ & 1993 & $0.13 \pm 0.02 \pm 0.05$ \\
OPAL $[14]$ & 1993 & $0.143_{-0.021}^{+0.022} \pm 0.007$ \\
DELPHI $[15]$ & 1993 & $0.121 \pm 0.016 \pm 0.004 \pm 0.004$ \\
UA1 [16] & 1991 & $0.148 \pm 0.029 \pm 0.017$ \\
CDF $[17]$ & 1991 & $0.176 \pm 0.027($ stat. $) \pm 0.022($ syst. $) \pm 0.032($ model $)$ \\
\hline \hline
\end{tabular}

Table 2.1: Mixing parameter, $\chi$.

jet charge $Q_{j e t}$ is defined by

$$
Q_{j e t}=\frac{\sum_{i} q_{i}\left|\vec{p}_{i} \vec{e}_{j}\right|^{\kappa}}{\sum_{i}\left|\vec{p}_{i} \vec{e}_{j}\right|^{\kappa}}
$$

where $\vec{p}$ is the particle momentum, $q_{i}$ its charge and $\vec{e}_{j}$ the unit vector in the direction of the jet. $\kappa$ is a calibration constant of the order of unity and was obtained from Monte Carlo studies. The resulting mixing parameter $\chi$ using the jet charge technique are consistent with the ones using dilepton events but have larger uncertainties.

Results of the mixing parameter, $\chi$, from different experiments are summarized in Table 2.1.

The previous CDF result [17] is based on $e \mu$ events in $2.7 \mathrm{pb}^{-1}$ of data collected during 1988-89 run. The major improvement of the present analysis from the previous one are:

- Almost three times $e-\mu$ events are collected in this experiment. This reduces the statistical uncertainty by $1 / \sqrt{3}$.

- Background $e-\mu$ events from direct $c \bar{c}$ production and also sequential semileptonical decays are reduced by requiring large transverse momenta for leptons with respect to the jet axis.

- By using data from the new muon detector, the uncertainty in estimating the fake lepton event fraction is reduced. 

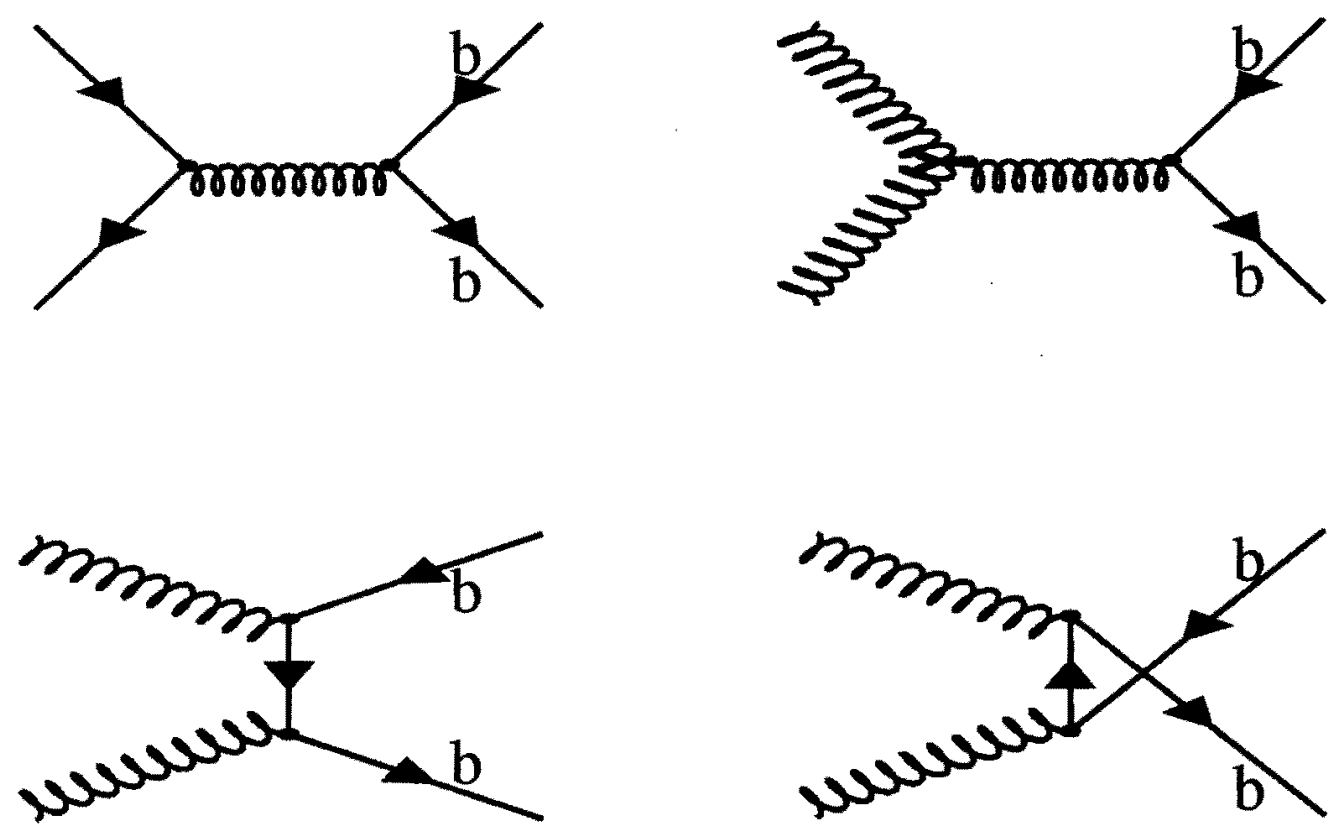

Figure 2.1: Feynman diagrams for lowest order $b \bar{b}$ production 

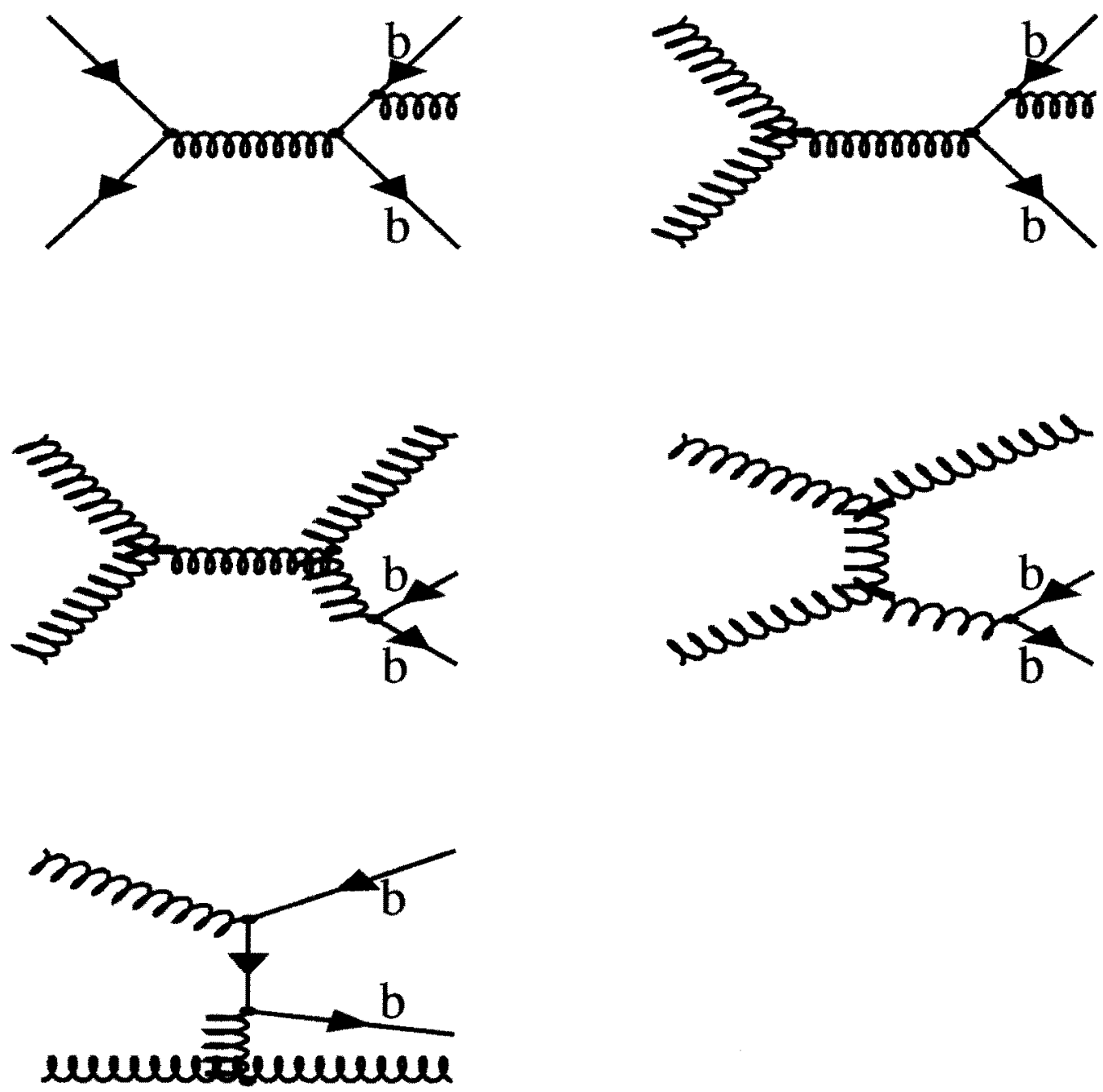

Figure 2.2: The example of Feynman diagrams for next leading order $b \bar{b}$ production 


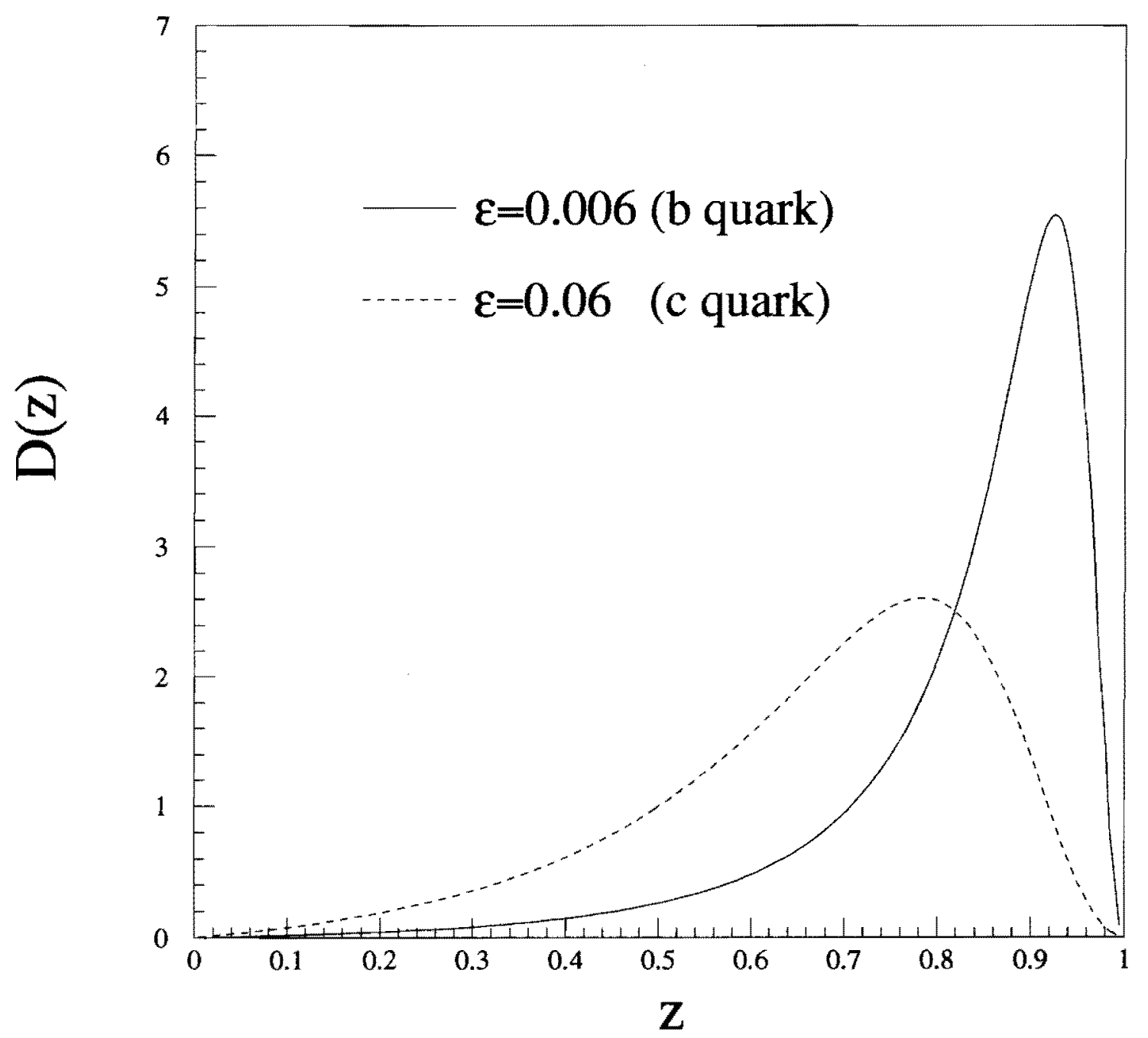

Figure 2.3: Fragmentation functions for $b$ and $c$ quarks. 


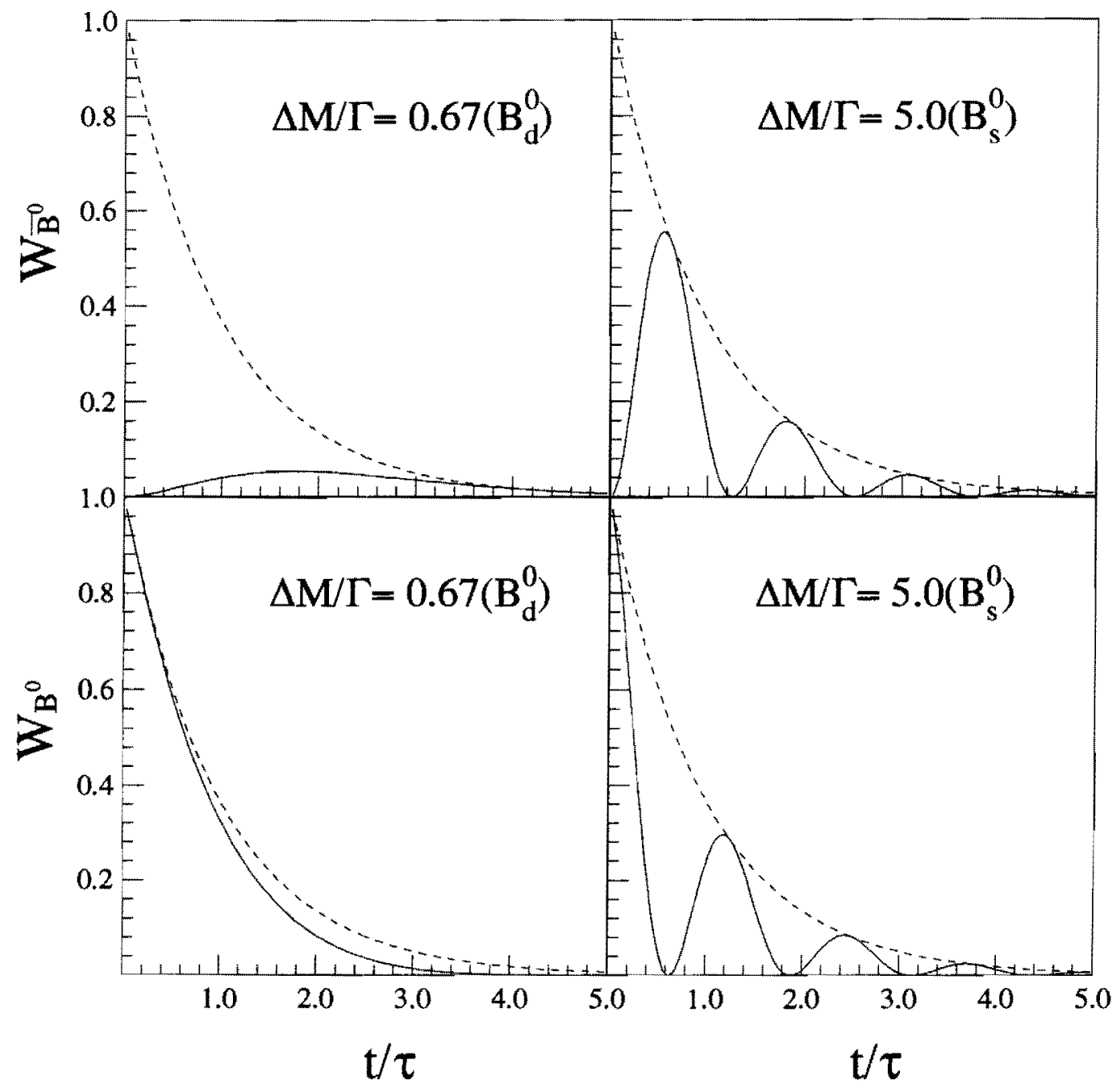

Figure 2.4: The solid lines show time dependent probabilities of finding $B^{0}$ and $\bar{B}^{0}$ in an initially pure $B^{0}$ state for $B_{d}$ and $B_{s}$ mesons. The dotted lines show the total decay probabilities. We assume $\Delta M / \Gamma=0.67(5.0)$ for the $B_{d}^{0}\left(B_{s}^{0}\right)$ meson. 


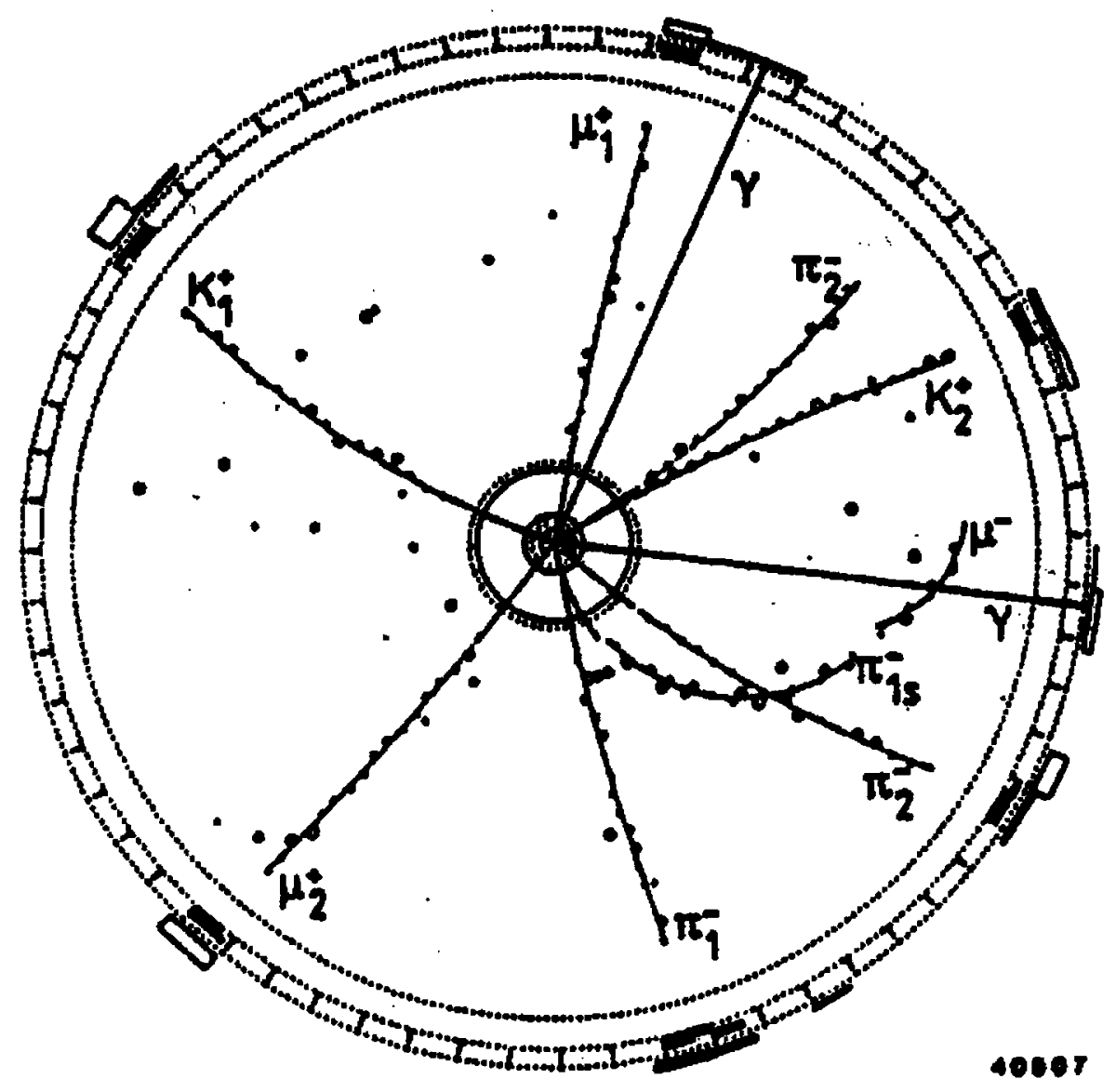

Figure 2.5: The completely reconstructed event consisting of the decay $\Upsilon(4 S) \rightarrow B^{0} B^{0}$. 


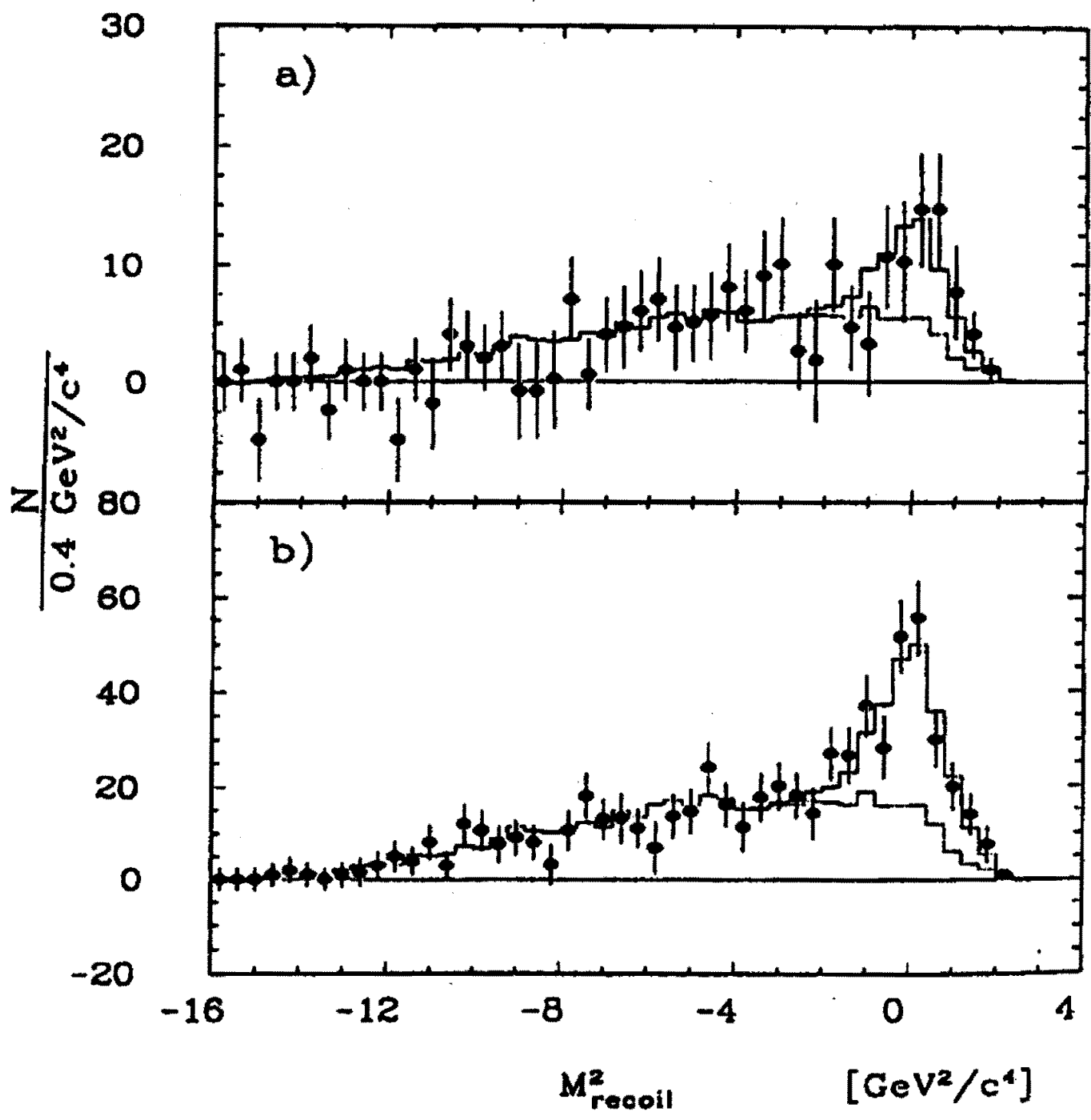

Figure 2.6: $M M^{2}$ spectra for $l^{+} \pi^{-}$(points with error) for events with an additional lepton with momentum $1.4<p_{l}<2.5$ : background (dotted histogram) and the result of the fit (full histogram) a) for like-sign leptons; $b$ ) for opposite-sign leptons. 


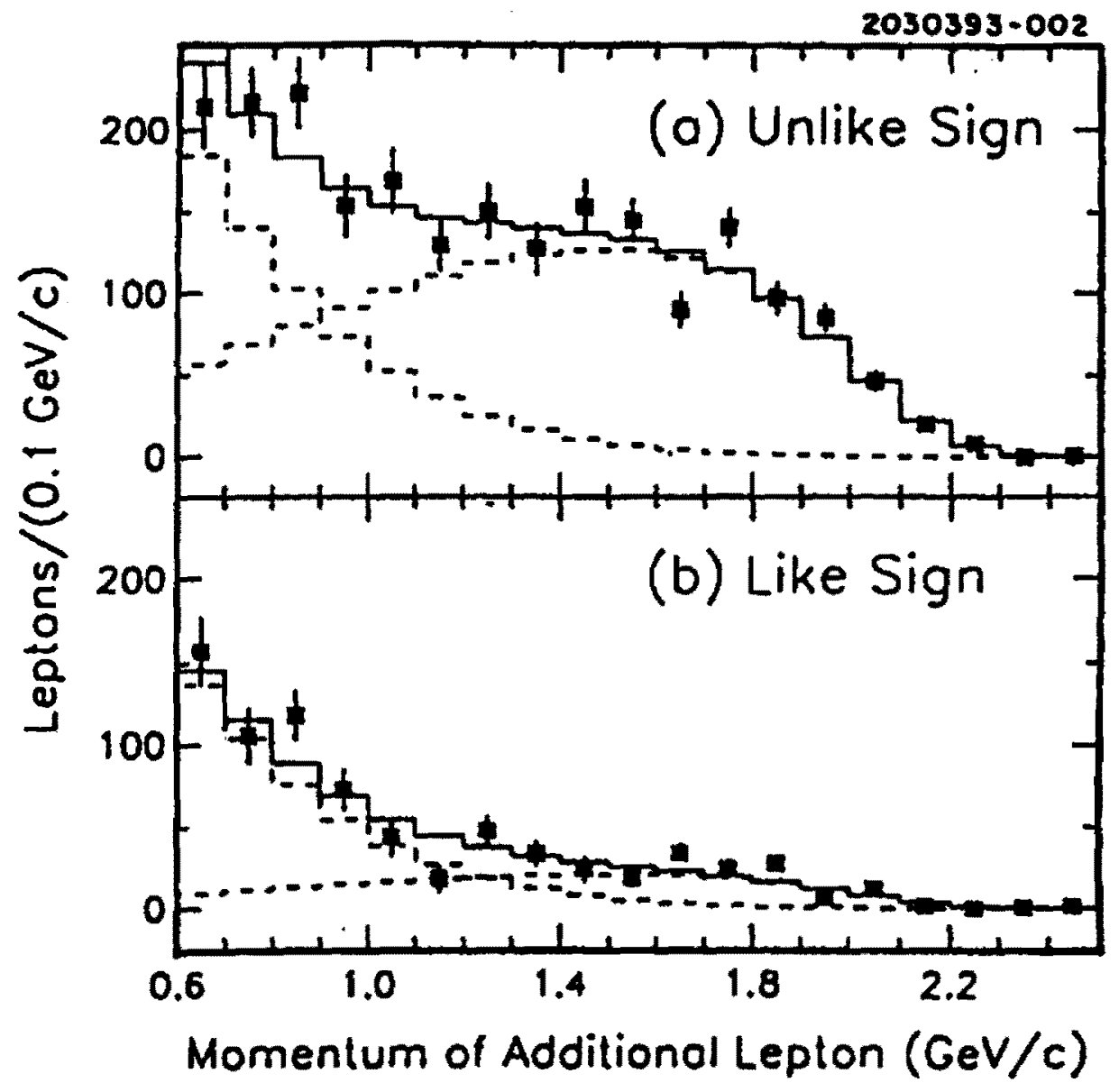

Figure 2.7: Efficiency corrected spectrum of additional leptons in tag events, for a) opposite-sign and $b$ ) same-sign leptons. Superimposed are fits (solid) to a primary lepton spectrum (dashed) plus a secondary spectrum (dash-dot). 


\section{Chapter 3}

\section{Apparatus}

This experiment was performed at the Fermi National Accelerator Laboratory (Fermilab) in Batavia, Illinois, U.S.A. using the Tevatron accelerator which gave proton-antiproton collisions at a center-of-mass energy of $1.8 \mathrm{TeV}$. Particles which were produced by the collisions were detected by the Collider Detector at Fermilab (CDF). The Tevatron accelerator and CDF detector are described below emphasizing what we used in our analysis.

\subsection{Tevatron}

The Tevatron accelerator produces the world highest energy proton-antiproton collisions at a center-of-mass energy of $1.8 \mathrm{TeV}$. Six accelerators of which the Tevatron accelerator consists are illustrated in Fig. 3.1. First, negatively charged hydrogen ions are injected to a DC voltage accelerator, Cockcroft-Walton electrostatic accelerator (C-W), and are accelerated to $750 \mathrm{keV}$. These particles are accelerated to $200 \mathrm{MeV}$ in 500 foot-length linear accelerator (LINAC). The two electrons are then stripped off the hydrogen ions, leaving bare protons, which are injected into the booster ring, a synchrotron with a diameter of 500 feet. The protons are accelerated up to $8 \mathrm{GeV}$ in the booster ring and are injected to the main ring. The main ring, a synchrotron with a diameter of two 
kilometers, is composed of water-cooled magnets. After being accelerated to $150 \mathrm{GeV}$ in the main ring, protons are injected to the Tevatron ring which is the same diameter with the main ring. The Tevatron ring is composed of superconducting magnets and can accelerate protons to $900 \mathrm{GeV}$.

Protons accelerated to $150 \mathrm{GeV}$ in the main ring are also used to initiate production of antiprotons. Every two seconds, approximately two trillion protons are accelerated to $120 \mathrm{GeV}$ in the main ring and then directed to an antiproton production target. Of the many particles and antiparticles of different types produced in collisions the protons make in the target, about ten million antiprotons are collected in a debuncher ring. The captured beam of antiprotons, circulating in the debuncher ring, is then made more dense by a process called stochastic cooling. The antiprotons are then transferred to the accumulator ring. The antiprotons merged into a single beam, cooled further, and stored in the accumulator ring. The debuncher and accumulator rings operate at $8 \mathrm{GeV}$. The antiprotons are accelerated by the main ring and the Tevatron ring in the opposite direction against the protons. Protons and antiprotons are collected into 6 bunches, so that a beam crossing occurs every $3.5 \mu \mathrm{sec}$.

The 1992-1993 run (RUN-Ia) started in April 1992 and ended in May 1993. The Tevatron provided about $25 \mathrm{pb}^{-1}$ and the CDF has collected an integrated luminosity of $\int L d t=20 \mathrm{pb}^{-1}$.

\subsection{Collider Detector at Fermilab}

The Collider Detector at Fermilab (CDF) is a general purpose detector located at the $B 0$ interaction region of the Tevatron ring, where collisions between protons and antiprotons occur. A perspective view of the CDF detector is shown in Fig. 3.2. The detector consists of 2 parts: the central detector and the forward and backward detectors. Figure 3.3 shows the location of each detector component. The tracking chambers consist of the silicon vertex detector (SVX), the vertex chamber (VTX) and the central tracking 
chamber (CTC). They are azimuthally symmetric around the beam axis. The solenoidal magnet which surrounds the tracking chambers generates $1.4 \mathrm{~T}$ magnetic field along the beam axis. The calorimeter used to measure energy of particles surrounds the solenoidal magnet. The calorimeter consists of the central electromagnetic calorimeter (CEM), the central hadronic calorimeter (CHA), the endwall hadron calorimeter (WHA), the plug electromagnetic calorimeter (PEM), the plug hadronic calorimeter (PHA), the forward electromagnetic calorimeter (FEM) and the forward hadron calorimeter (FHA). The muons which passed through the calorimeters reach muon chambers. The muon detectors consists of the central muon system (CMU), the central muon upgrade (CMP), the central muon extension (CMX) and the forward muon system (FMU). The beam-beam counters (BBC) are placed in front of the forward and backward calorimeters and are used for luminosity monitor and trigger. The detectors and detector components most relevant to this analysis are briefly reviewed in the following sections.

The CDF coordinate system is taken as follows. The origin is the center of detector, the nominal collision point, and the $\mathrm{z}$-axis is the proton beam direction. The $\mathrm{x}$-axis points to the outside of the Tevatron ring and the $y$-axis points toward vertically upward, so as to make a right-handed coordinate system. The azimuthal angle $\phi$ is an angle measured from the positive $\mathrm{x}$-axis toward the positive $\mathrm{y}$-axis. The pseudo-rapidity $\eta$ is defined by $\eta \equiv-\ln (\tan \theta / 2)$, where the polar angle $\theta$ is an angle from the proton beam direction ( the positive $z$-axis). The radius $r$ is a distance from the beam axis in $x-y$ plane. The central detector and the forward (backward) detector cover regions $10^{\circ}<\theta<170^{\circ}$ and $\theta<10^{\circ}\left(\theta>170^{\circ}\right)$, respectively.

\subsubsection{Beam-beam counters}

The beam-beam counters (BBC) are planes of scintillation counters which are located at the front face of each of the forward and the backward calorimeters. A beam's-eye view of the one of the BBC's is shown in Fig. 3.5. They provide a minimum-bias trigger for the detector in addition to a role of the luminosity monitor. The timing resolution of 
the counters is less than 200 psec. They cover the polar angle region from $0.32^{\circ}$ to $4.47^{\circ}$ along either the horizontal or vertical directions, corresponding to a pseudo-rapidity range of 3.24 to 5.90 .

\subsubsection{Tracking detectors}

\section{Silicon Vertex detector (SVX) [18]}

The SVX consists of two cylindrical modules placed end-to-end with their axes coincident with the beamline. The total longitudinal coverage is $51 \mathrm{~cm}(2 \times 25.5 \mathrm{~cm}$ with $2.15 \mathrm{~cm}$ gap). Because $p \bar{p}$ interactions are spread along the beamline with standard deviation $\sigma \sim 30 \mathrm{~cm}$, the geometrical acceptance of the SVX is about $60 \%$ for $p \bar{p}$ interactions. Each module consists of four radial layers (numbered 0-3 from beam pipe) of silicon strip detector. The inner and outer layers are at radii of $3.005 \mathrm{~cm}$ and $7.866 \mathrm{~cm}$, respectively. Figure 3.6 shows a view of one cylindrical module of the SVX. For layers 0,1 and 2 of the silicon strip detector, the strips are on a $60 \mu \mathrm{m}$ pitch along the beam axis. For layer 3 they are on a $55 \mu \mathrm{m}$ pitch. The resolution for high $p_{T}$ tracks is $\sim 10 \mu \mathrm{m}$ after correction of the alignment of the SVX geometry by using real track data.

\section{Vertex chamber (VTX)}

The VTX is designed primarily to determine the event vertex position in the longitudinal $z$ direction. The VTX provides tracking information up to a radius of $22 \mathrm{~cm}$ and $|\eta|<3.25$. The VTX consists of vertical planes of drift chambers along the $z$ direction, with each individual $z$ slice being radially divided into octants. The individual chamber octants have sense wires arranged tangentially to the beam, providing track hit information mainly in $(r, z)$ coordinates. The typical resolution of vertex point turned out to be about $1 \mathrm{~mm}$ in the $z$ direction. 
Central Tracking Chamber (CTC) [19]

The CTC is a $1.3 \mathrm{~m}$ radius and $3.2 \mathrm{~m}$ long cylindrical drift chamber which gives precise momentum measurements in the angular region $40^{\circ}<\theta<140^{\circ}(-1<\eta<1)$. In this region the momentum resolution is better than $\delta p_{T} / p_{T}^{2} \leq 0.002(\mathrm{GeV} / \mathrm{c})^{-1}$. The chamber contains 84 layers of the sense wires grouped into 9 "superlayers". Five of the superlayers consist of 12 axial sense wires; four stereo superlayers consist of 6 sense wires tilted by $\pm 3^{\circ}$ relative to the beam direction. Figure 3.7 shows an endplate of the chamber displaying the $45^{\circ}$ tilt of the superlayers to the radial direction to correct for the Lorentz angle of the electron drift in the magnetic field.

Leading electrons drift into the azimuthal direction with a maximum drift distance being less than $40 \mathrm{~mm}$, corresponding to a drift time $800 \mathrm{~ns}$. The axial superlayer signals give a picture of the event in $r-\phi$ plane. The stereo superlayer signals give a picture of the event in $r-z$ plane in cooperation with the axial superlayer signals.

\subsubsection{Calorimeters}

In order to contain showers produced by particles from $p \bar{p}$ collision at the nominal interaction point a "tower" geometry was chosen for all calorimeters as shown in Fig 3.4. The coverage of the calorimeter towers in $\eta-\phi$ space is shown in Fig 3.8. Each tower has an electromagnetic shower counter in front of a corresponding hadron calorimeter, so that one can make a detailed comparison of electromagnetic to hadronic energy on a tower-by-tower basis. The towers are projective, i.e., they point at the interaction region, and are 0.1 units of $\eta$ wide by $15^{\circ}$ (central region) or $5^{\circ}$ (plug and forward regions) in $\phi$. The physical size of a tower ranges from about $24.1 \mathrm{~cm}(\eta) \times 46.2 \mathrm{~cm}(\phi)$ in the central region to $1.8 \mathrm{~cm} \times 1.8 \mathrm{~cm}$ in the forward region. Geometric coverages in $\eta$ direction and absorption thickness for calorimeters are summarized in Table 3.1. The absorption thicknesses in Table 3.1 are given in radiation lengths, $X_{0}$, for the electromagnetic calorimeters or in pion absorption lengths, $\Lambda_{a b s}$, for the hadron calorimeters. 


\begin{tabular}{l|c|r}
\hline \hline $\begin{array}{c}\text { Calorimeter } \\
\text { component }\end{array}$ & $\begin{array}{c}\text { Geometric coverage } \\
\text { in } \eta \text { direction }\end{array}$ & $\begin{array}{c}\text { Absorption } \\
\text { thickness }\end{array}$ \\
\hline CEM & $0.0<|\eta|<1.1$ & $18 X_{0}$ \\
PEM & $1.2<|\eta|<2.4$ & $18-21 X_{0}$ \\
FEM & $2.4<|\eta|<4.2$ & $18 X_{0}$ \\
\hline CHA & $0.0<|\eta|<0.8$ & $4.7 \Lambda_{a b s}$ \\
WHA & $0.8<|\eta|<1.3$ & $4.5 \Lambda_{a b s}$ \\
PHA & $1.3<|\eta|<2.4$ & $5.7 \Lambda_{a b s}$ \\
CHA & $2.4<|\eta|<4.2$ & $7.7 \Lambda_{a b s}$ \\
\hline \hline
\end{tabular}

Table 3.1: Geometric coverage in $\eta$ direction and absorption thickness for calorimeter components.

Central calorimeters: Central electromagnetic calorimeter (CEM), Central hadron calorimeter (CHA) and Endwall hadron calorimeter (WHA)

The central calorimeters cover a polar angle region of $39^{\circ}<\theta<141^{\circ}(|\eta|<1.1)$. It is azimuthally segmented into $15^{\circ}$ wedges. There are 48 wedges in all, 24 on each side of the $z=0$ plane. A perspective view of one central calorimeter wedge is shown in Fig. 3.9. Each wedge has an electromagnetic part and a hadronic calorimeter part, and is subdivided along the $z$-axis into ten projective towers, numbered from 0 to 9 , where tower 0 is at $90^{\circ}$ polar angle. The size of the central calorimeter tower is approximately $15^{\circ}$ in $\phi$ and 0.11 units in $\eta$.

The CEM [20] consists of 31 layers of $5 \mathrm{~mm}$ polystyrene scintillator interleaved with 30 layers of $1 / 8$ inch lead. Total thickness of CEM module is approximately eighteen radiation lengths. Using the test beam electron with an energy range of $15-100 \mathrm{GeV}$, the energy resolution of the CEM was measured [21] as

$$
\frac{\Delta E}{E}=\frac{13.5 \%}{\sqrt{E \sin \theta}} \quad(E \text { in } \mathrm{GeV})
$$

where the $\sin \theta$ reflects the change of the sampling thickness seen by the electron emitted at a polar angle $\theta$. 
The CEM has a gas proportional wire chamber with cathode strip embedded approximately at the EM shower maximum, 5.9 radiation lengths including the solenoid. The central strip chamber (CES) consists of wires along the beam direction for $\phi$ measurements and cathode strips perpendicular to wires for $z$ measurements. The CES provides a precise determination of shower center and shower shape. The position resolution is about $2.2 \mathrm{~mm}$ in the wire $(\phi)$ view and $1.4 \mathrm{~mm}$ in the strip view for $50 \mathrm{GeV} / \mathrm{c}$ electrons.

The CHA [22] consists of 32 layers of $10 \mathrm{~mm}$ plastic scintillator interleaved with 32 layers of $25 \mathrm{~mm}$ iron. The WHA consists of 15 layers of $10 \mathrm{~mm}$ plastic scintillator interleaved with 15 layers of $50 \mathrm{~mm}$ iron. Total material thickness is 4.7 units of pion absorption lengths for $\mathrm{CHA}$ and 4.5 units for the WHA. The $\mathrm{CHA}$ has 9 projective towers numbered from 0 to 8 along $z$-axis. The WHA has 6 projective towers numbered from 6 to 11 along $z$-axis. The number $6(7,8)$ tower of the CHA occupies the same tower in $\eta-\phi$ plane as the number $6(7,8)$ of WHA in the same wedge. The energy resolution for the CHA is given by

$$
\frac{\Delta E}{E}=\frac{75 \%}{\sqrt{E \sin \theta}} \quad(E \text { in } \mathrm{GeV})
$$

from test beam pion data with an energy range of 10-150 GeV [23]. From the same beam test, the energy resolution for WHA is given by

$$
\frac{\Delta E}{E}=\frac{80 \%}{\sqrt{E}} \quad(E \text { in } \mathrm{GeV}) .
$$

Plug calorimeters: plug electromagnetic calorimeter (PEM) and plug hadronic calorimeter (PHA)

The PEM [24] is an annular shaped detector which covers a polar angles region of $10^{\circ}<\theta<32^{\circ}$. This polar angle region covers the end of the CTC. The PEM is a gas-based calorimeter using conductive plastic proportional tube arrays $(7 \times 7 \mathrm{~mm})$ interleaved with $0.27 \mathrm{~cm} \times 34$ lead absorber panels and read out by cathode pads. Shower profiles can be measured with a resolution of $2 \mathrm{~mm}$ by this calorimeter $[24,25]$. The 
tower segmentation in the plug calorimeter region is $\Delta \eta \sim 0.9$ and $\Delta \phi \sim 5^{\circ}$, finer than that in the central region. The energy resolution was measured in an electron test beam with an energy range of $20-200 \mathrm{GeV}$ to be [26]

$$
\frac{\Delta E}{E}=\frac{28 \%}{\sqrt{E}} \quad(E \text { in } \mathrm{GeV})
$$

The PHA is located behind the PEM, forming a partial conical shape. The PHA uses steel plates as absorber, with similar conductive plastic proportional tubes. Total material thickness is 5.7 units of pion absorption lengths. The energy resolution for the PHA was measured as

$$
\frac{\Delta E}{E}=\frac{132 \%}{\sqrt{E}}
$$

by the test beam pions with an energy range of $40-200 \mathrm{GeV}$ [27].

Forward calorimeters: Forward electromagnetic calorimeter (FEM) and forward hadron calorimeter (FHA)

The FEM is located behind the BBC's on both sides of the detector. The FEM's employ a gas-based calorimeter very similar to the $P E M$, with $0.48 \mathrm{~cm} \times 30$ lead absorber panels and tower segmentation $\Delta \phi \sim 5^{\circ}$ and $\Delta \eta \sim 0.1$. The coverage extends from 2.2 to 4.2 in $\eta$. The FEM position resolution ranges from $1 \mathrm{~mm}$ to $4 \mathrm{~mm}$, depending on the location.

The FHA covers the forward region from 2.3 to 4.2 in $\eta$ with an additional 7.7 pion absorption lengths behind the FEM. The FHA preserves the tower geometry of the FEM.

\subsubsection{Muon detectors}

The central muon detectors are located behind the calorimeters because electrons, photons and hadrons are absorbed inside calorimeters. The central muon detectors are divided into several components, each covering a different section. The central muon system (CMU) and central muon upgrade (CMP) cover a pseudo-rapidity region of $|\eta|<0.6$. The central muon extension (CMX) covers a pseudo-rapidity region of $0.65<|\eta|<1.0$. 
The coverage of the muon chambers is shown in the Fig. 3.10. The CMP and CMX were added just before RUN-Ia. The CMP is used for confirmation of muon track stubs in the CMU and it also fills up crack in $\eta=0$ and wedge gaps of the CMU. The CMX extends the coverage of the CMU to about $\eta \sim 1.0$.

\section{Central muon system (CMU) [28]}

The CMU is divided into 24 wedges $\left(\Delta \phi=15^{\circ}\right)$ forming an approximate cylinder around the beamline. The CMU is divided into 2 parts at $\eta=0$. Each CMU wedge contains a total of 48 drift chambers, each $2.3 \mathrm{~m}$ long, arranged axially along the $z$ direction. Figure 3.11 shows the layout of a CMU wedge; note there are significant cracks in acceptance between the wedges. The diagram in Fig. 3.12 shows a cross sectional view of a $5^{\circ}$ section of the $\mathrm{CMU}$, with a typical muon track passing through the four layers of drift cells.

The individual drift cells shown in Fig. 3.12 measure $63.5 \mathrm{~mm} \times 26.8 \mathrm{~mm}$ in cross section, with a sense wire in the center, and are operated with a $50 \% / 50 \% \mathrm{Ar}-\mathrm{C}_{2} \mathrm{H}_{6}$ gas mixture. A muon track stub is formed by measuring the drift time for each of the four sense wires along the muon path. The left-right ambiguity of the track is resolved by staggering the wires along the radial line. The chambers were operated in limited stream mode, allowing a measurement of the $z$ position by measuring the relative charge deposited at both ends of the sense wire.

\section{Central muon upgrade (CMP) [29]}

The CMP is mounted on four flat planes around the central barrel detector, with a layer of steel ( $\sim 3$ absorption lengths) beyond the CMU. On the top and bottom of the detector, the CMP is attached to the steel of the solenoid return yoke. On both side of the detector, the CMP planes are attached to walls of steel. The tubes are continuous across the CMU $\eta=0$ crack. The chambers are of fixed length in $z$ and form the box around the central detector, therefore the actual pseudo-rapidity coverage varies with 
azimuth as shown in Fig. 3.10.

The CMP consists of $864 \mathrm{drift}$ tubes with the size of $2.5 \mathrm{~cm} \times 15 \mathrm{~cm} \times 640 \mathrm{~cm}$. The CMP drift tubes are half cell staggered and use the same argon/ethane gas mixture as used in the CMU. Figure 3.13 shows a schematic view of a CMP tube. Like the CMU, there are four layers of drift cells, and muon track stubs can be reconstructed in the $r-\phi$ plane by measuring the drift times in the four layers. However, no $z$ information on the muon track is available from the CMP unlike CMU. The position resolution of the tube is about $300 \mu \mathrm{m}$.

\section{Central muon extension (CMX) [29]}

The CMX occupies the surface of a cone (see Fig 3.2). The CMX are free standing, just beyond the central barrel region. The CMX extends pseudo-rapidity coverage from 0.65 to 1.0 .

The CMX consists of $864 \times 2 \mathrm{drift}$ tubes, which are of the same structure as the CMP tube. The tubes are sandwiched between two layers of scintillation counters which are used for triggering.

\subsubsection{Trigger}

The CDF trigger system consists of three levels [30, 31, 32]. The level 1-3 triggers reduce the high rate of $\sim 280 \mathrm{kHz}$ in the 1992-1993 CDF run of $p \bar{p}$ interactions to a rate of $\sim 6 \mathrm{~Hz}$ at which events could be recorded on tape. The level 1-3 triggers consist of a logical OR of several requirements of electrons, photons, muons, missing energy, jets, taus, and select events based on physics interests. The level 3 trigger is made up of 48 Silicon Graphics computers, each containing two event buffers, plus an array of service hardware to push the data into and out of 96 buffers. Each event is sent to a single buffer, and so that level 3 triggers can be processed up to 48 separate events in parallel, with another 48 events meanwhile being loaded to the secondary buffers. Here we describe a low $P_{T}$ dilepton trigger, which is called "JPSI STREAM 1". We used 
events which passed this trigger in this analysis.

\section{Level 1 trigger}

All level 1 triggers require a coincidence between hits in the forward and backward BBC scintillators to select physical reactions.

The level 1 trigger for low $P_{T}$ dilepton events consists of level 1 dielectron trigger, level 1 dimuon trigger and level 1 electron-muon trigger. The level 1 dielectron (dimuon) trigger is made by a pair of level 1 electron (muon) triggers. The level 1 triggers for electron-muon events are generated by a pair of a level 1 low- $E_{T}$ calorimeter trigger and a level 1 muon trigger.

The level 1 low- $E_{T}$ calorimeter trigger is produced from analog signals coming from phototubes in the central calorimeters. The trigger is segmented in detector $\eta-\phi$ plane by $\Delta \phi=15^{\circ}$ and $\Delta \eta=0.2$. The actual segmentation provided by the calorimeters is reduced by summing fast analog signals in order to reduce the large quantity of signals to a manageable level, resulting in a total of 2048 trigger towers, half electromagnetic and half hadronic. The analog calorimeter signals are corrected for pedestal offsets and gain variations. The level 1 low- $E_{T}$ calorimeter trigger required that a trigger tower have the transverse EM energy above $4 \mathrm{GeV}$. No correction is made for possible variations in the $z$ vertex position at this stage.

The level 1 muon trigger is produced from a fast analog measurement of momentum of muons in the CMU and a coincidence signal between chambers in alternate layers in the CMP. The momentum of muons is measured by a difference of drift times between layer 2 and layer 4 of CMU chambers (See Fig. 3.12). The difference of the drift time is a function of momentum of muons. The level 1 muon trigger requires the momentum to be greater than $3.3 \mathrm{GeV} / \mathrm{c}$. 


\section{Level 2 trigger}

The level 2 trigger for low $P_{T}$ dilepton events consists of a level 2 dielectron trigger, a level 2 dimuon trigger and a level 2 electron-muon trigger. The level 2 dielectron (dimuon) trigger is made by a pair of level 2 electron (muon) triggers. The level 2 trigger for electron-muon events is generated by a pair of a level 2 low- $E_{T}$ calorimeter trigger and a level 2 muon trigger.

Electromagnetic energy clusters are formed from trigger towers by hardware processors at this stage. The clustering algorithm used is that, first, a trigger tower with transverse EM energy greater than $4 \mathrm{GeV}$ is searched, then four adjacent towers are examined one by one to be added to the seed tower if its transverse EM energy is greater than $3.6 \mathrm{GeV}$, and each attached trigger tower is taken as a seed tower and the same procedure is repeated until no more trigger tower is found to be added. A ratio of total (EM + HAD) transverse energy to transverse EM energy is calculated from electromagnetic and hadronic energies in the clusters. The level 2 electron trigger requires the ratio be less than 1.125 .

The CTC tracks are reconstructed in two dimensions by a hardware processor called Central Fast Tracker (CFT) [33]. The CFT processes fast timing information from the CTC to identify a high $P_{T}$ track in the $r-\phi$ plane by comparing the CTC hits

with predetermined patterns. The CFT has a momentum resolution of $\Delta P_{T} / P_{T}^{2} \sim$ $0.035(\mathrm{GeV} / \mathrm{c})^{-1}$, with a high efficiency [33]. The level 2 electron trigger requires the momentum of an associated track with the EM cluster to be greater than $4.8 \mathrm{GeV} / \mathrm{c}$.

The level 2 muon trigger requires matching between the CFT track and a stub in the muon detectors. The momentum of the CFT track for the level 2 muon trigger is required to be greater than $3 \mathrm{GeV} / \mathrm{c}$.

\section{Level 3 trigger}

The level 3 trigger for electron and muon requires the following.

- Level 3 electron 
- Transverse energy: $E_{T}>5 \mathrm{GeV}$

- Transverse momentum: $P_{T}>3 \mathrm{GeV} / \mathrm{c}$

- Lateral shower sharing: $L_{\mathrm{shr}}>0.3$

- Lateral shower shape: $\chi_{\text {strip }}^{2}<15$

- Difference between a measured shower position in the CES and an extrapolated CTC track at the CES: $\Delta X<6 \mathrm{~cm}$ and $\Delta Z<10 \mathrm{~cm}$

- Level 3 muon

- Transverse momentum: $P_{T}>3 \mathrm{GeV} / \mathrm{c}$

- Difference between a CMU stub position and an extrapolated CTC track at the entrance of the CMU chambers: $\Delta X<16 \mathrm{~cm}$ and $\Delta Z<16 \mathrm{~cm}$

Because the level 3 trigger uses same code as offline analysis, the lateral shower sharing, lateral shower shape, position matching will be defined and described later.

The level 3 trigger for low $P_{T}$ dilepton events consists of a level 3 dielectron, a level 3 dimuon trigger and a level 3 electron-muon trigger. The level 3 dielectron (muon) trigger is generated by a pair of the electron (muon) triggers. The level 3 electron-muon trigger is generated by a pair of a level 3 electron and a level 3 muon trigger.

\subsubsection{Data acquisition system}

The CDF data aquisition system (DAQ) [34] employs a FASTBUS-based multi-level network. The CDF DAQ has multiple partitions each of which covers one or more independent detector sections so that the DAQ for different detector components can proceed in parallel. At the lowest level of the DAQ system, there are two major types of front end system. When an event is accepted by both level 1 and level 2 , the data from front end crates are digitized and read by scanner modules. All the calorimeters and the central muon system use the redundant analog bus-based information transfer (RABBIT) which are read by MX scanner [35]. Most tracking detectors use FASTBUS 
TDCs which are read by SSP scanners [36]. A few CAMAC modules are included in the event data stream and are read by an SSP scanner using a FASTBUS to CAMAC interface. Each scanner can buffer four events. This step in the DAQ pipeline is managed by the trigger supervisor (TS) FASTBUS module. The TS uses a combination of FASTBUS messages and dedicated control lines to provide flexible and efficient control of front end systems. Each partition is allocated a unique set of scanners and a trigger supervisor.

When all MX and SSP scanners have finished reading and buffering data for one event, the TS module sends a FASTBUS message to buffer manager (BFM) indicating that an event is available in a specified buffer. The BFM supervises dataflow from scanner modules to host VAX computers. The BFM initiates this dataflow by sending a FASTBUS message to event builder (EVB) instructing it to "Pull" an event from

the same buffer in all scanners of a specified detector partition. The EVB is a group of FASTBUS modules which can read, buffer and reformat complete events from any allowed partition of detector components. When the EVB has finished reading data from scanner buffer $N$, it sends a "Pull OK" message to the BFM which in turn notifies the TS that buffer $N$ is available for a new level 1 and level 2 trigger.

Under direction of the BFM, the EVB writes a complete event into a specified node in the level 3 processor farm (Silicon Graphics computers). Events accepted by the level 3 trigger are read by the buffer multiplexer executing on one or more computers in the VAX cluster. Event data can be logged to disk or tape.

\subsubsection{Luminosity measurement}

The luminosity can be obtained by counting the rate of a certain process of which cross section is known. We use inelastic $p \bar{p}$ events, and the BBC's to detect them. That is, we have

$$
L=\frac{R_{B B C}}{\sigma_{B B C}},
$$


where $L$ is the luminosity, $R_{B B C}$ is the BBC event rate, and $\sigma_{B B C}$ is the effective cross section visible to the BBC's. We estimate $\sigma_{B B C}$ at $1800 \mathrm{GeV}$ as follows.

We first calculate the corresponding quantity for the UA4 experiment at $546 \mathrm{GeV}$ $[37,38]$, using their absolute measurements of the total and elastic $p \bar{p}$ cross sections, to be $38.9 \pm 1.8 \mathrm{mb}$. A small correction due to the difference in the geometry of the trigger counters at CDF and UA4 is made in order to convert the above quantity into the corresponding CDF quantity at $546 \mathrm{GeV}, \sigma_{B B C}^{546}$. We then relate it to the value at $1800 \mathrm{GeV}, \sigma_{B B C}^{1800}$, by using the measurements of the Tevatron luminosities $\left(L_{a c c}\right)$ based on accelerator parameters, and the observed $\mathrm{BBC}$ rates $R_{B B C}$ at the two energies. They have a relation

$$
\frac{\sigma_{B B C}^{1800}}{\sigma_{B B C}^{546}}=\frac{R_{B B C}(1800)}{R_{B B C}(546)} \cdot \frac{L_{a c c}(546)}{L_{a c c}(1800)} .
$$

We obtain [39]

$$
\sigma_{B B C}^{1800}=46.8 \pm 3.2 \mathrm{mb} .
$$

With recent direct measurements of elastic and total cross sections by CDF collaboration [40], we are able to measure a direct measurement of the BBC cross section of

$$
\sigma_{B B C}=51.2 \pm 1.7 \mathrm{mb} .
$$

After accounting for possible backgrounds in the BBC's, we have a total uncertainty of $3.6 \%$ on the integrated luminosity. 


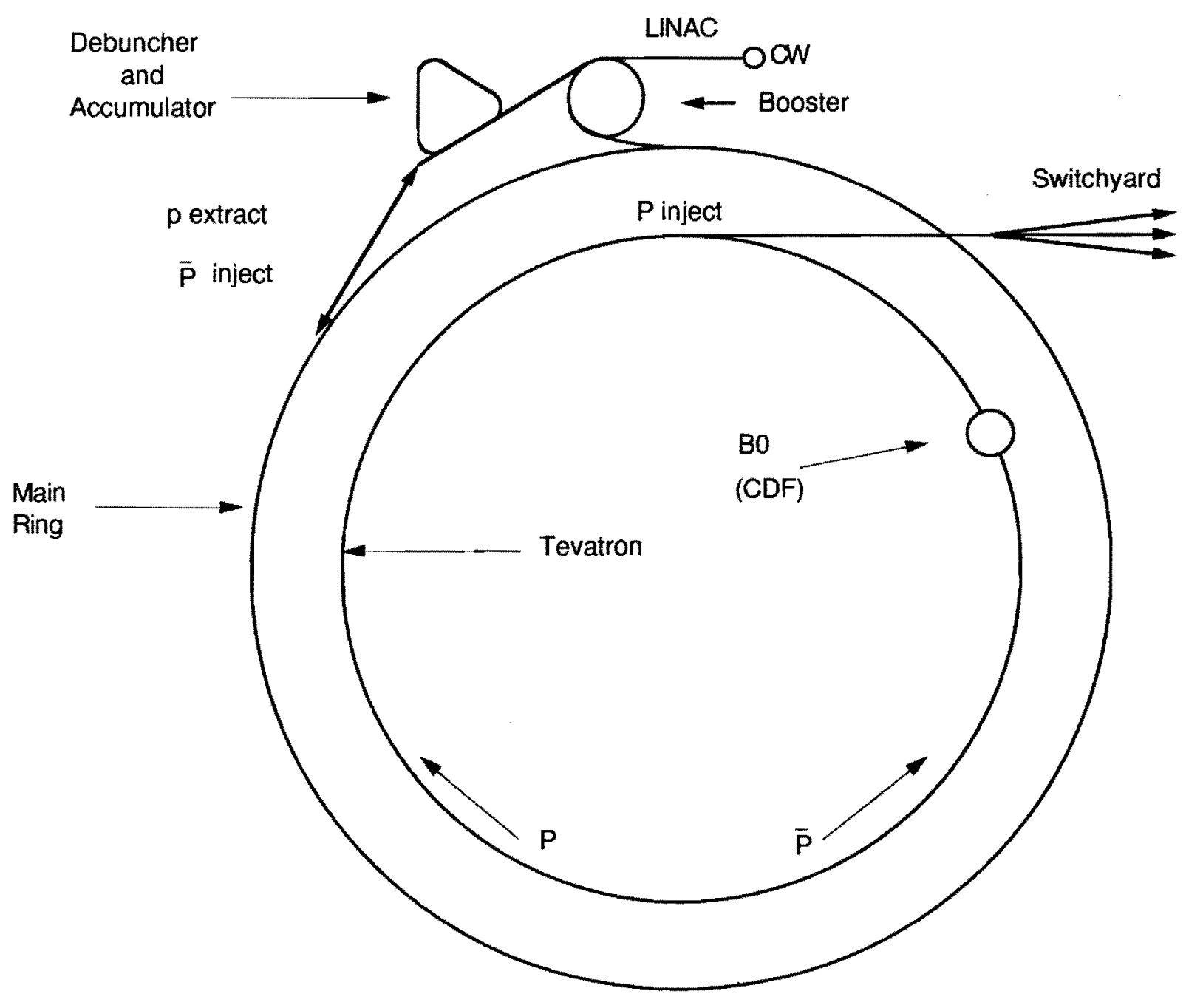

Figure 3.1: Diagram of the Tevatron accelerator complex. 


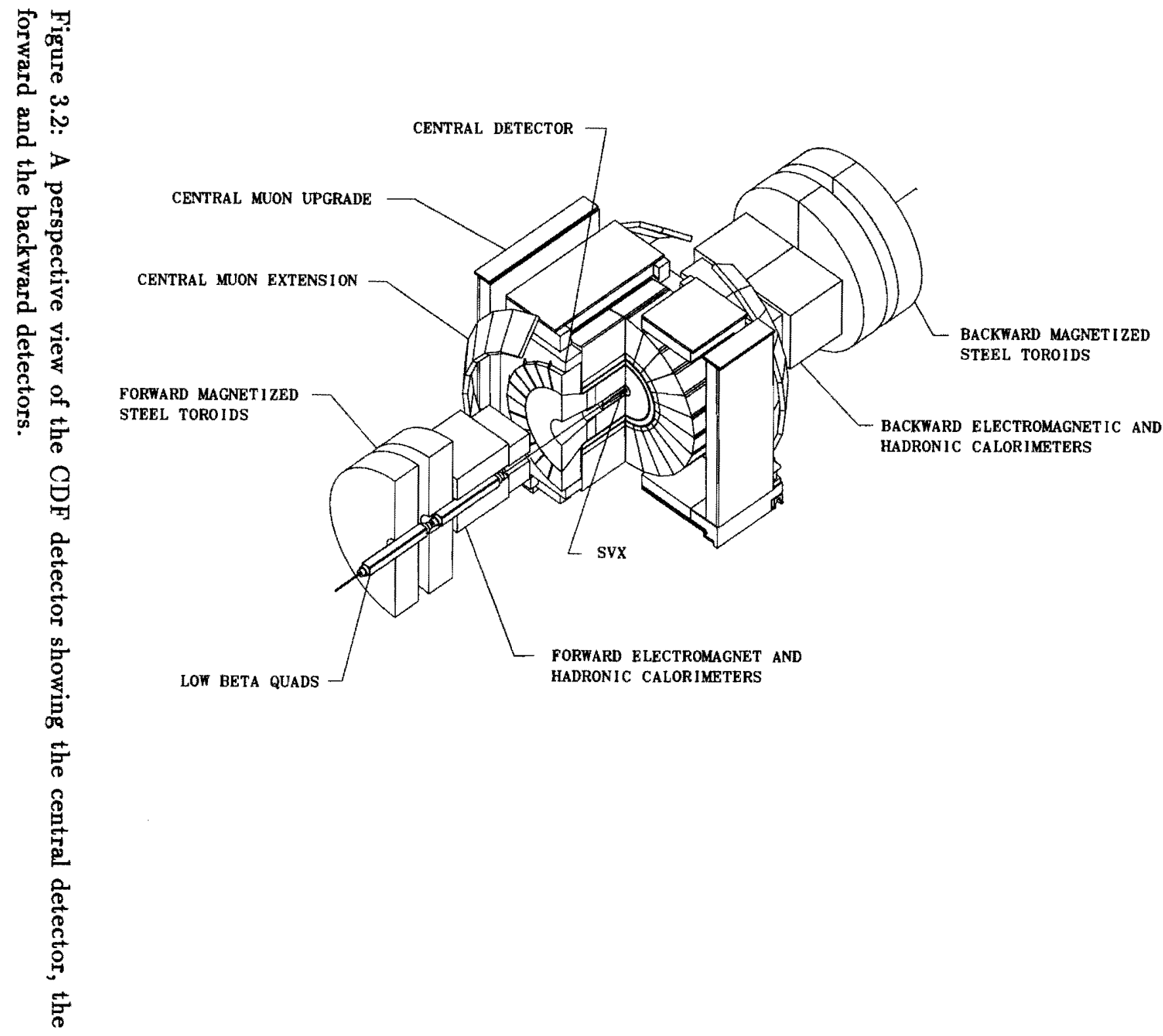




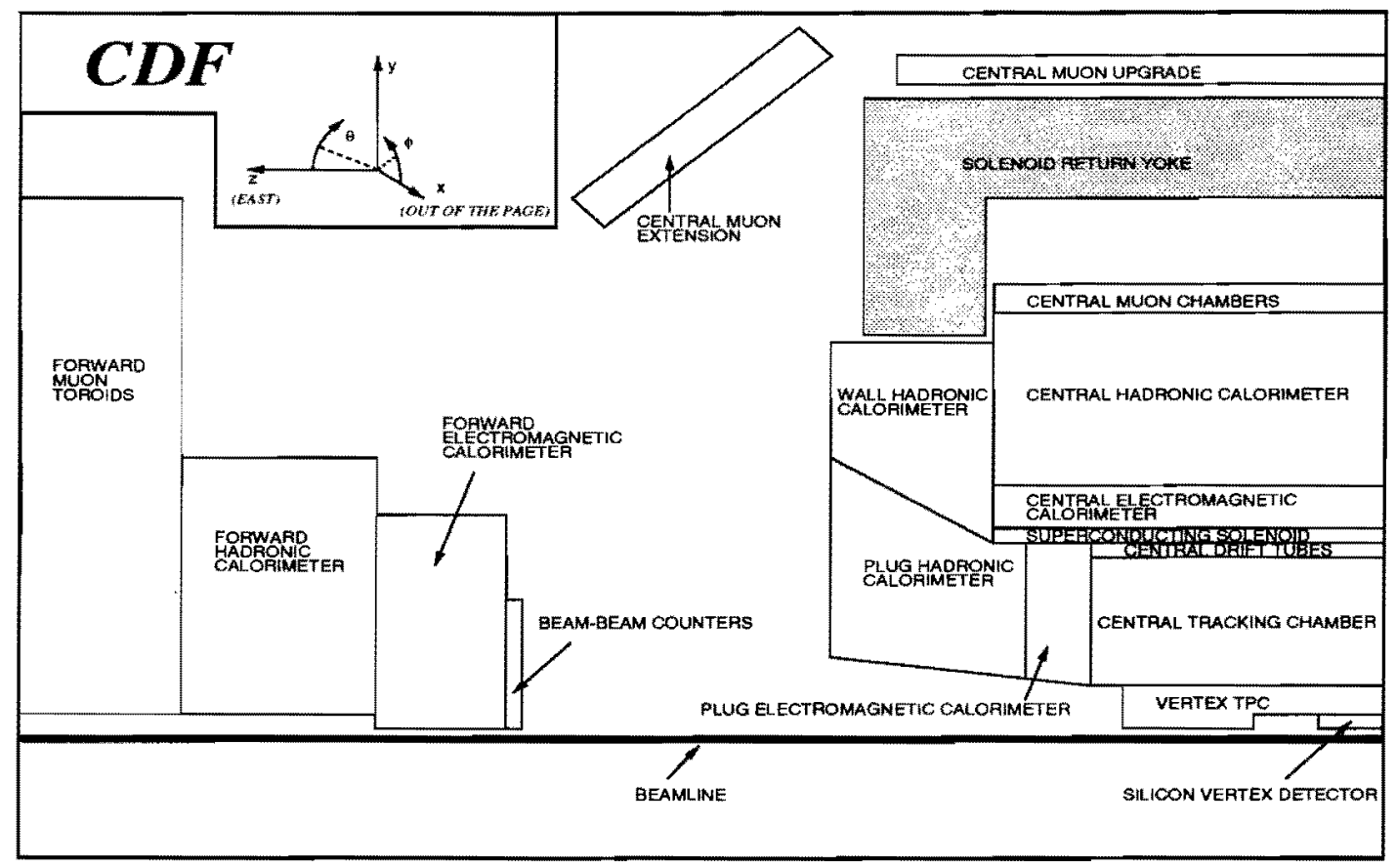

Figure 3.3: A side-view cross section of the CDF detector. The detector is forwardbackward symmetric about the interaction point. 


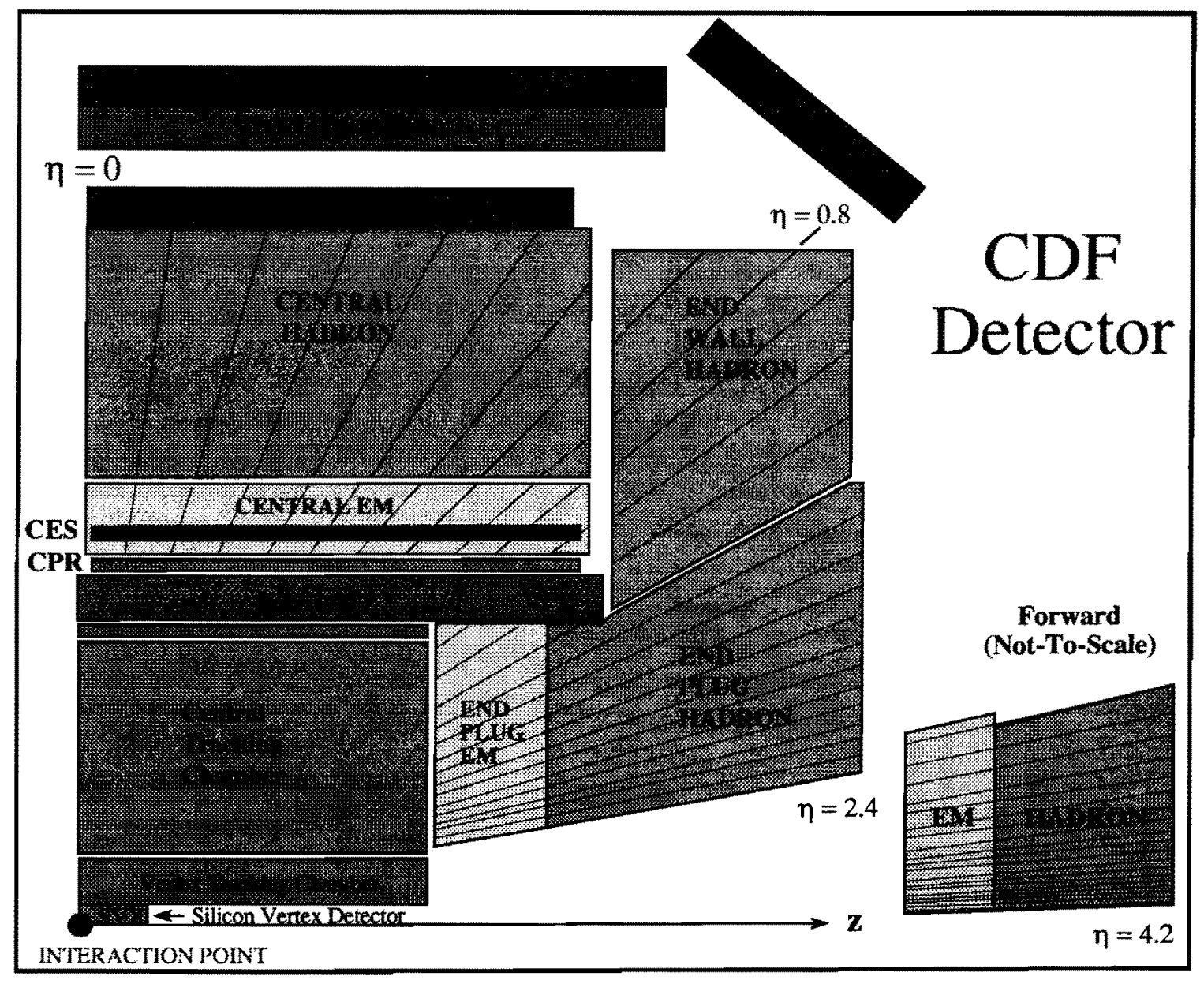

Figure 3.4: Cross-sectional view of the central detectors. 


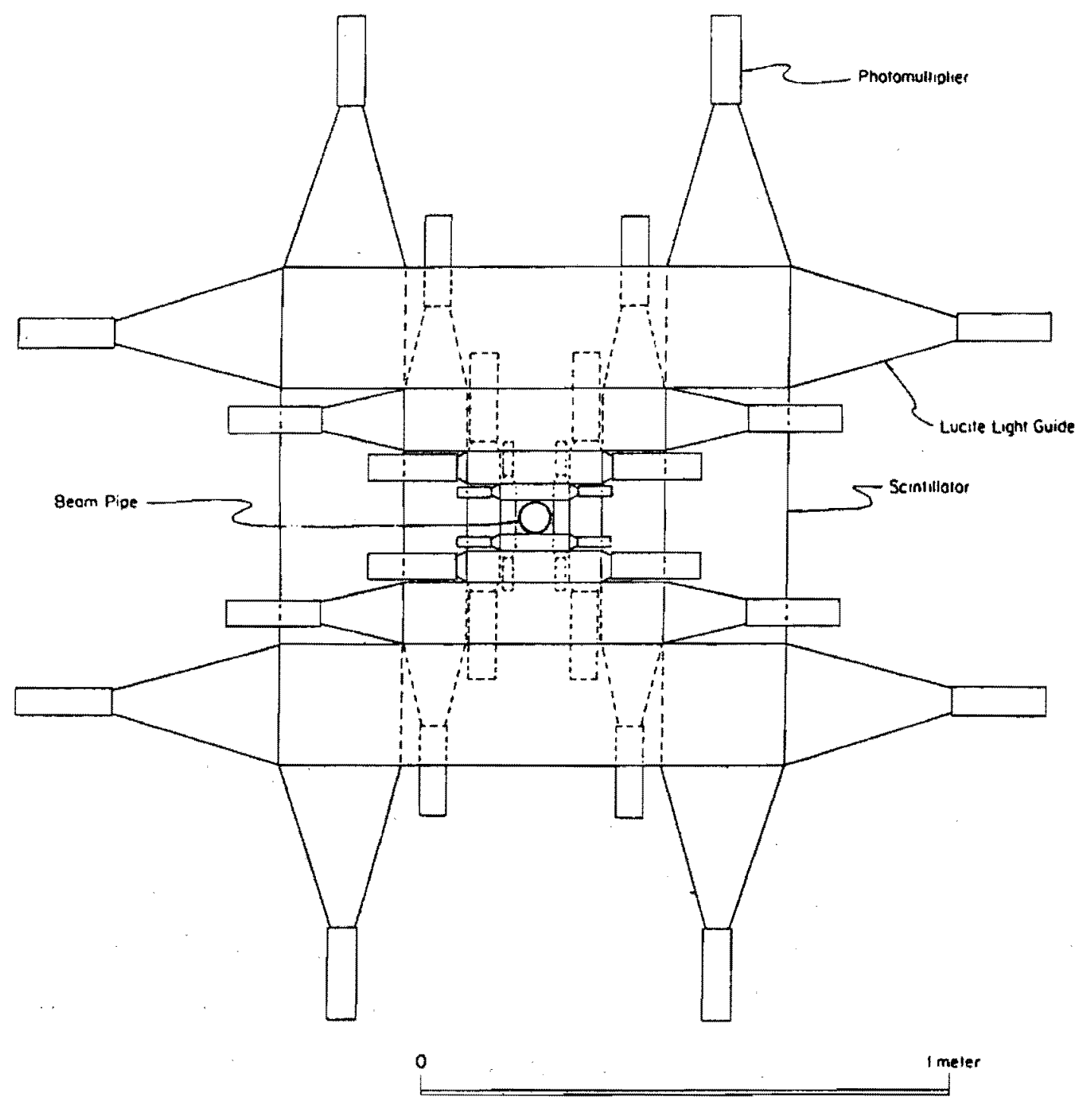

Figure 3.5: A beam's eye view of one of the beam-beam counter planes. 


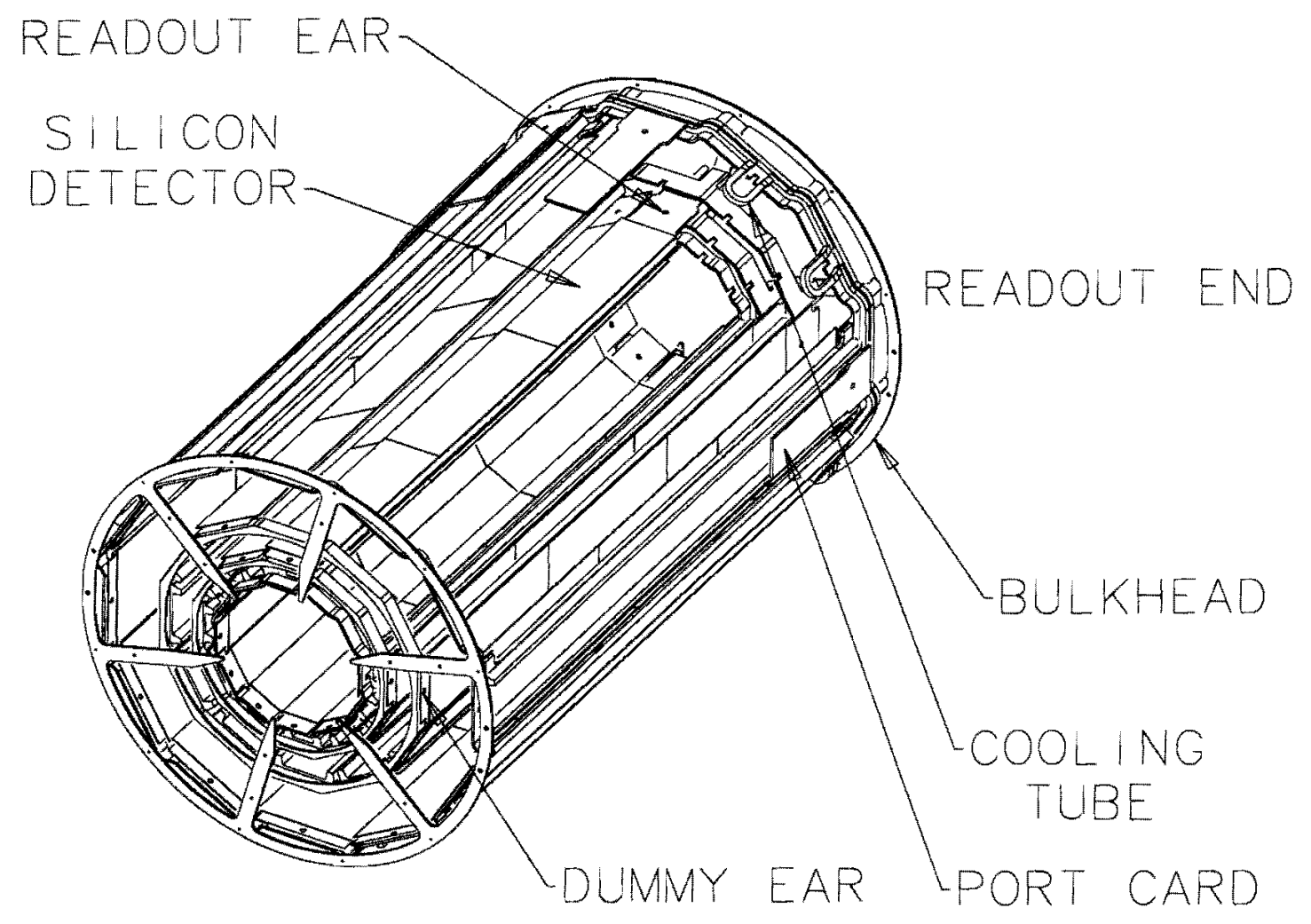

Figure 3.6: A perspective view of the SVX barrel. 


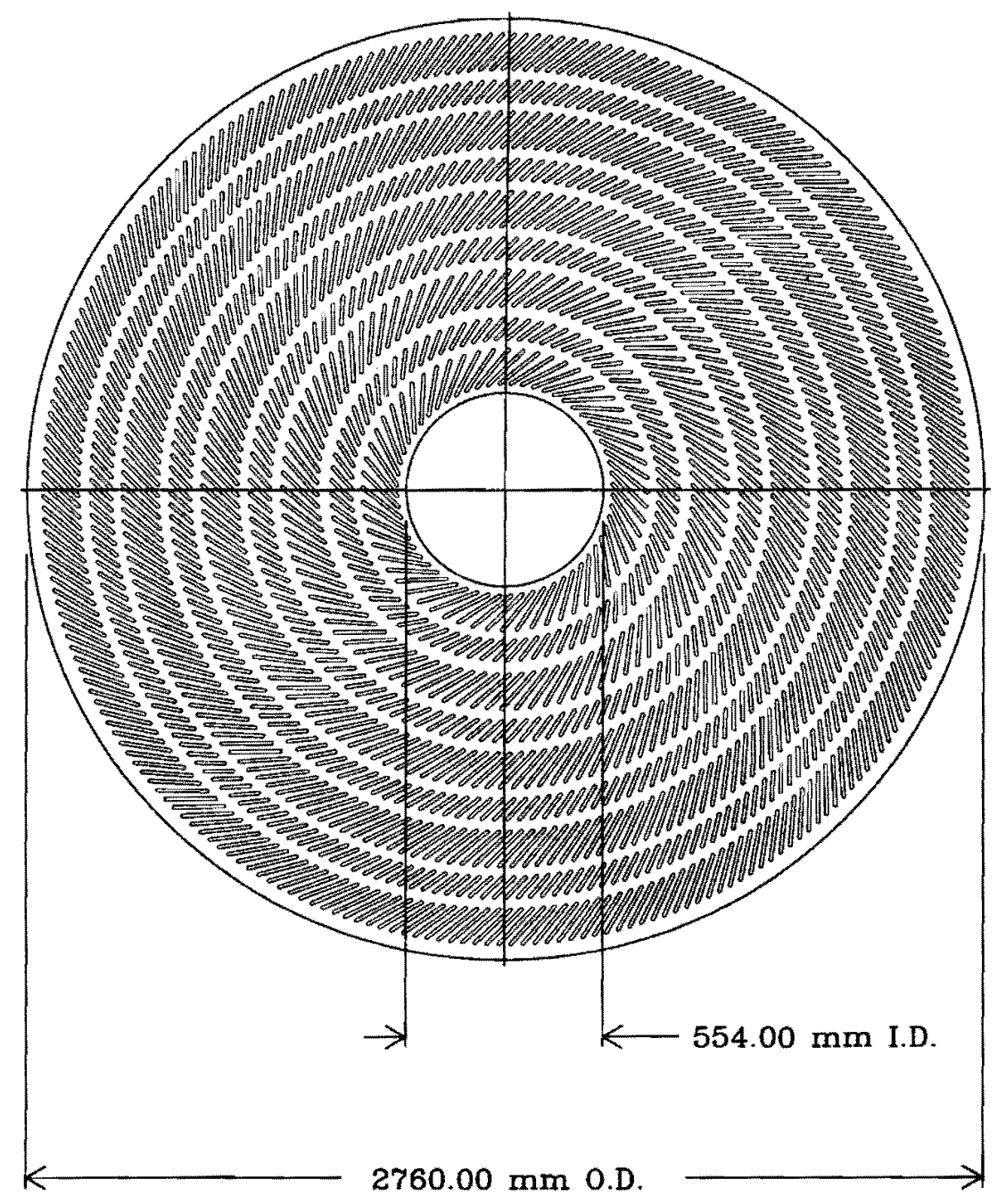

Figure 3.7: An endplate of the CTC showing the arrangement of the blocks which hold the 84 layers of sense wires. 


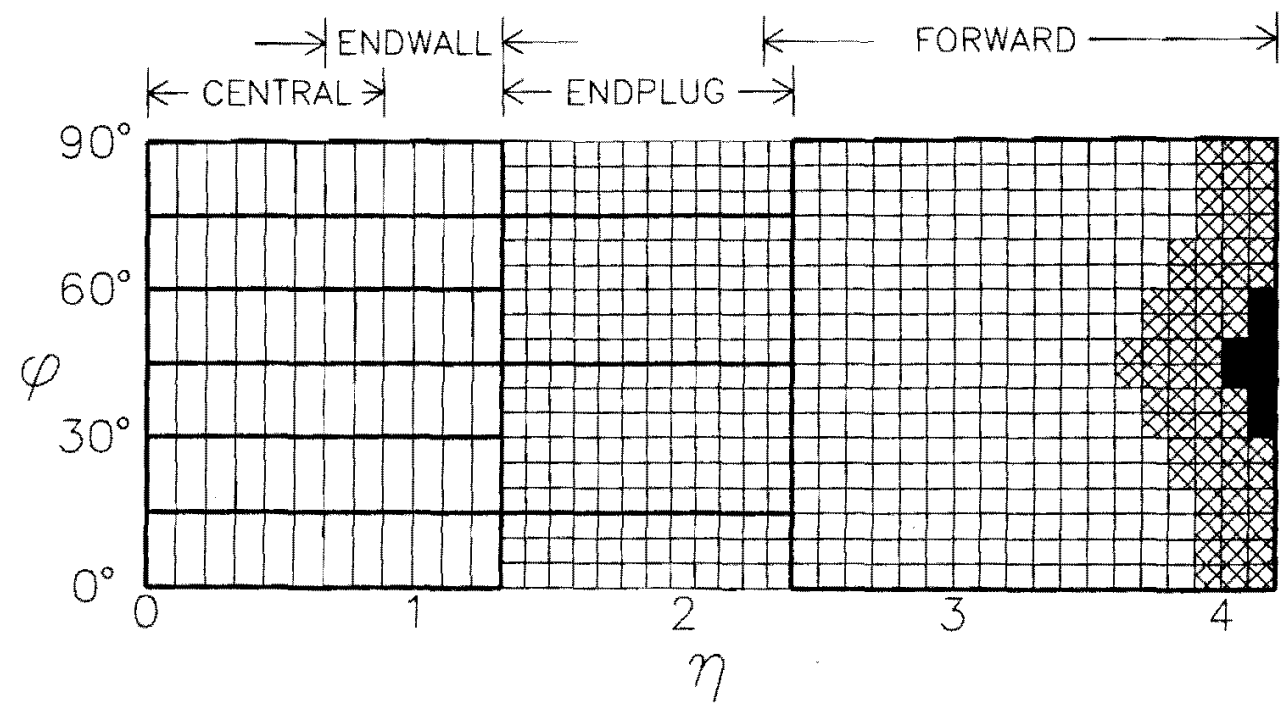

Ex Partial depth coverage only due to cut for low beta quadrupoles No coveroge

Figure 3.8: Hadron calorimeter tower in one of eight identical $\eta-\phi$ quadrants $(\Delta \phi=$ $\left.90^{\circ}, \eta>0\right)$. The heavy lines indicate module or chamber boundaries. The EM calorimeters have complete $\phi$ coverage out to $\eta=4.2$. 


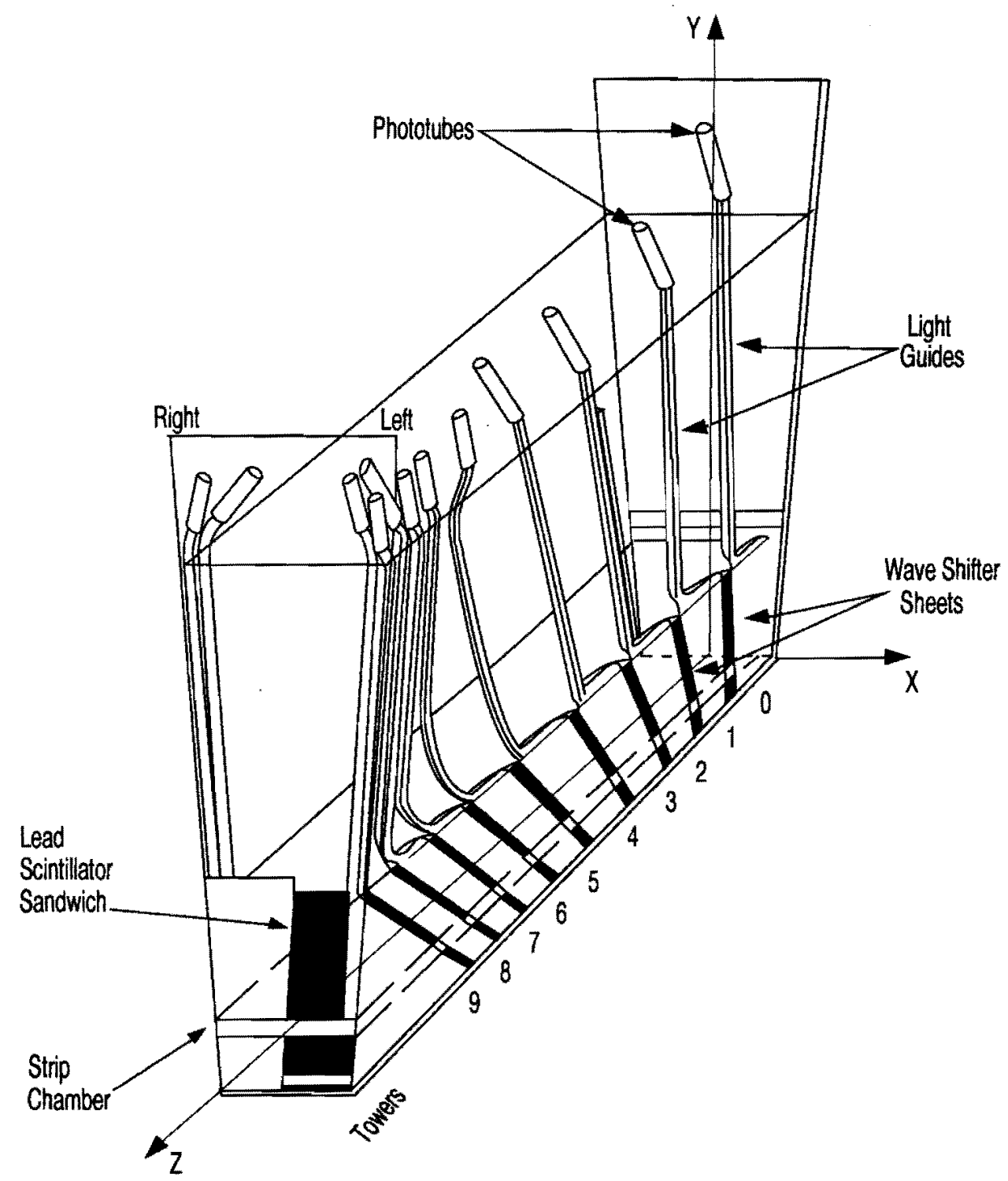

Figure 3.9: Schematic of a wedge module of the CDF central calorimeter showing the coordinate system as measured by the strip chamber used for test beam energy and mapping measurements. The $45^{\circ}$ end plate is in the $+z$ direction; the "left" of the module was defined as being in the $+x$ direction. 


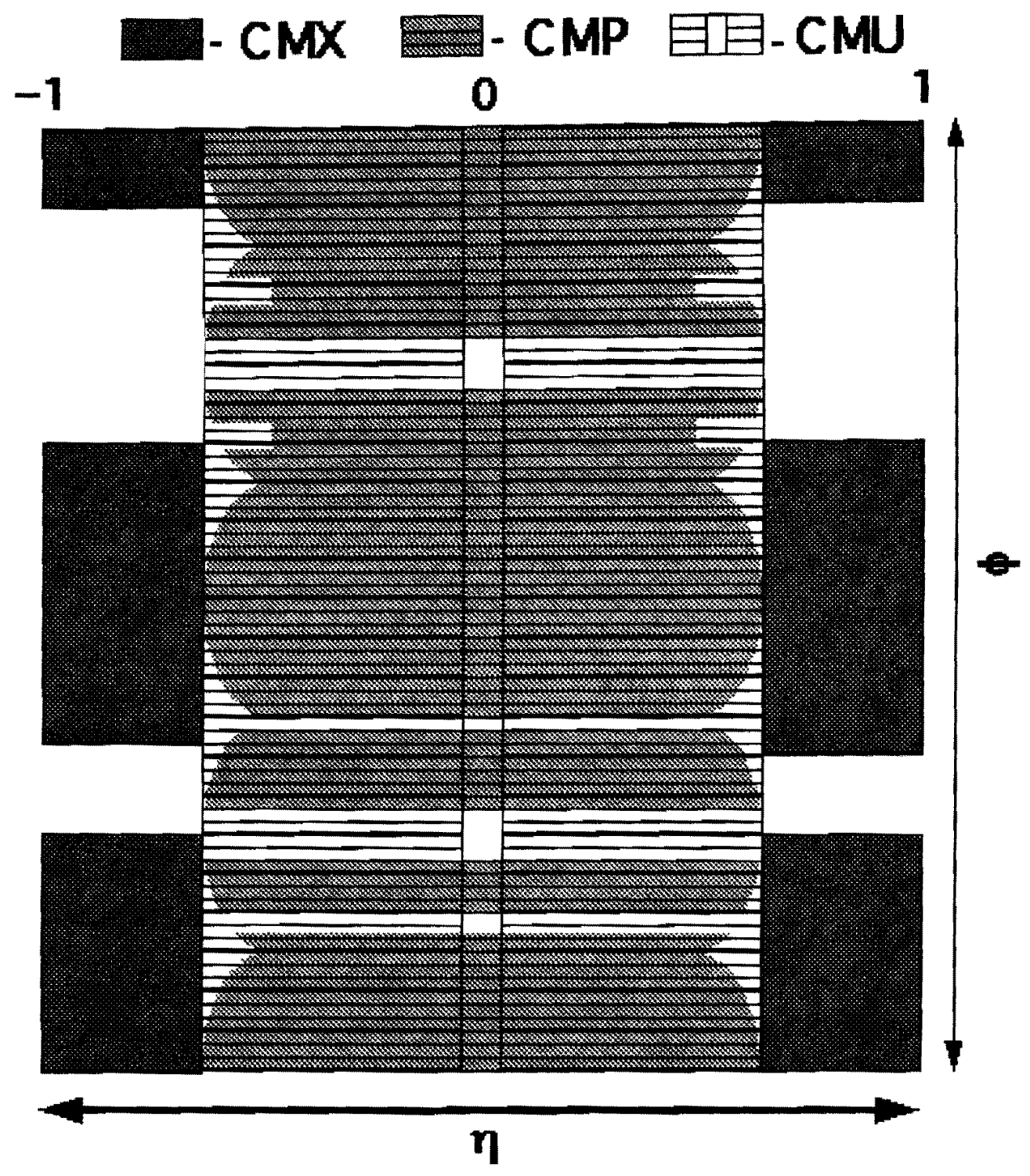

Figure 3.10: Map of muon eta-phi coverage. 


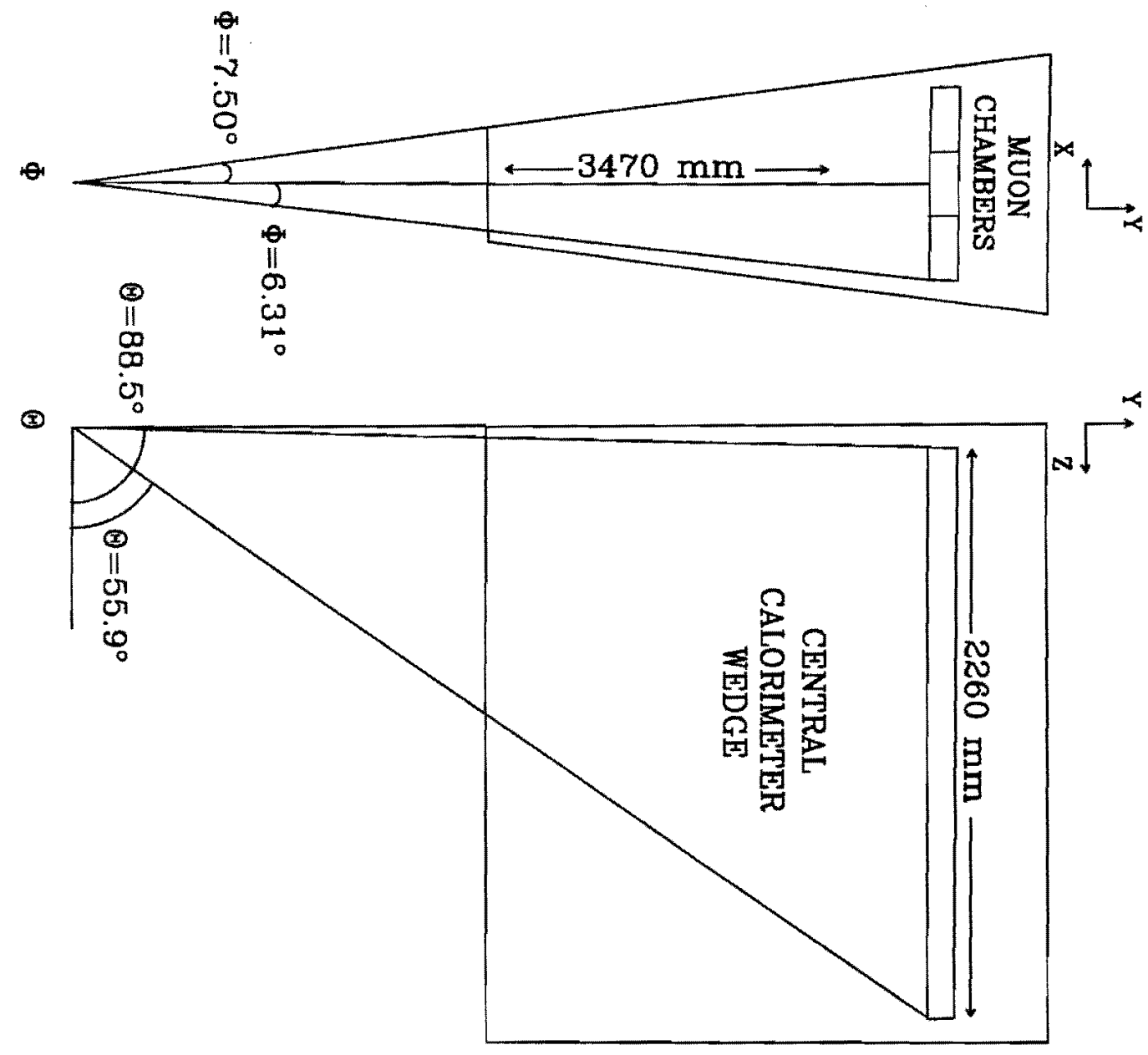

Figure 3.11: The layout of the central muon chambers in one of the central wedges. 


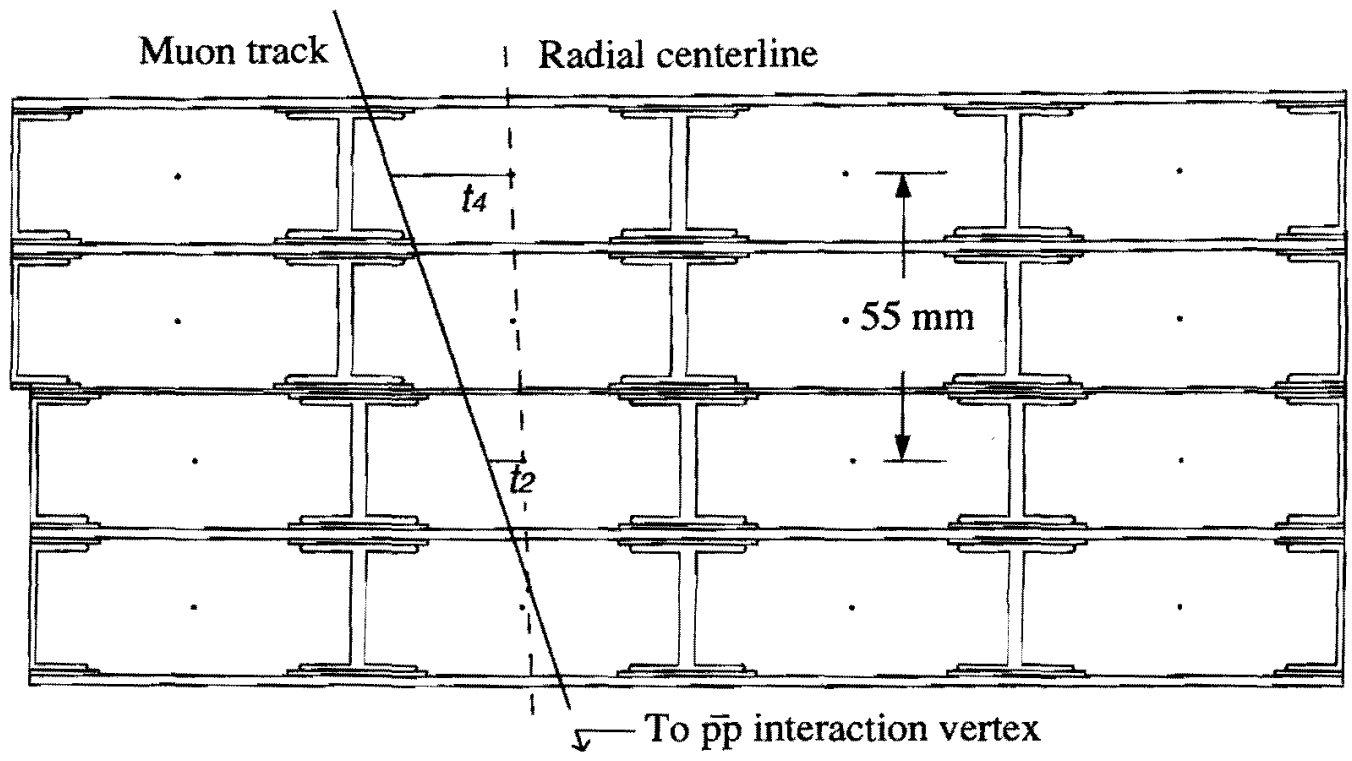

Figure 3.12: The arrangement of the four planes of central muon chambers in a view along the beam direction. The drift times $t_{2}$ and $t_{4}$ are used at the trigger level to determine a muon momentum cut off. 


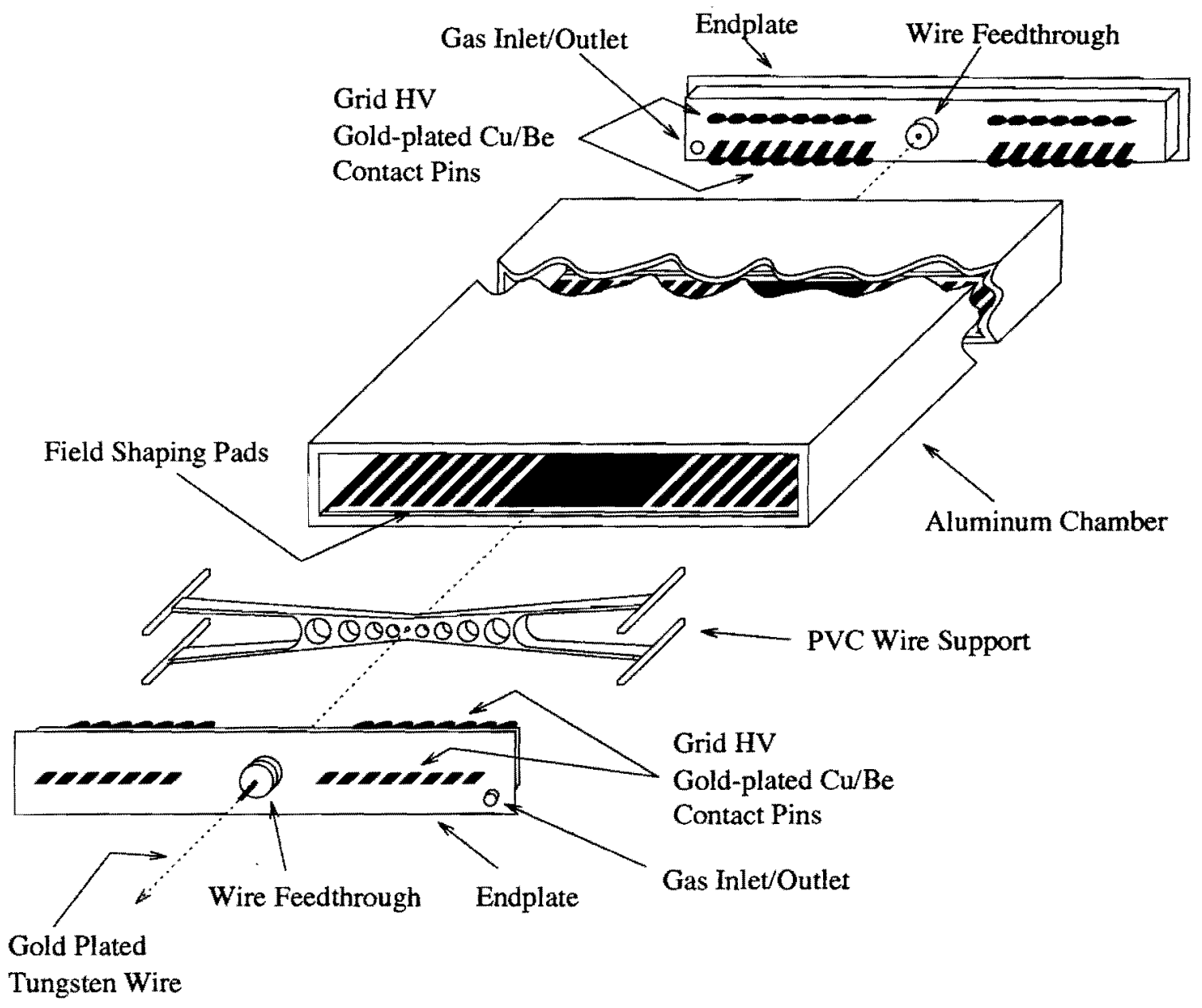

Figure 3.13: Schematic view of a CMP-CMX tube 


\section{Chapter 4}

\section{Analysis Outline and Data}

\section{Reduction}

\subsection{Outline of the analysis}

The mixing parameter, $\chi$, can be estimated from Eq. (2.35) in Chapter 1 in principle, but several complications arise in actual analysis including consideration of sequential decays and backgrounds.

The important decay processes of $b \bar{b}$ events in this analysis are listed in Table 4.1. In Process 1 , both $b$ and $\bar{b}$ quarks decay to leptons directly, which is mentioned in Chapter 1 . In Process $2(3)$, the $b(\bar{b})$ quark decays to a lepton directly and the other $\bar{b}(b)$ quark to

\begin{tabular}{|c|c|c|}
\hline$\left\{\begin{array}{l}b \\
\vec{b} \rightarrow l+X \\
\rightarrow l+X\end{array}\right.$ & & (Process 1 ) \\
\hline $\begin{cases}b & \rightarrow l+X \\
\bar{b} & \rightarrow \bar{c}+X\end{cases}$ & $\rightarrow \quad l+X$ & (Process 2) \\
\hline$\left\{\begin{array}{l}b \\
\bar{b} \rightarrow c+X\end{array}\right.$ & $\rightarrow l+X$ & (Process 3) \\
\hline
\end{tabular}

Table 4.1: Semileptonic decay processes. 


\begin{tabular}{|l||c|c|c||c|}
\cline { 2 - 5 } & $\begin{array}{c}\text { Process 1 } \\
\left\{\begin{array}{l}b \rightarrow l \\
\bar{b} \rightarrow l\end{array}\right.\end{array}$ & $\begin{array}{c}\text { Process 2 } \\
\left\{\begin{array}{l}b \rightarrow l \\
\bar{b} \rightarrow \bar{c} \rightarrow l\end{array}\right.\end{array}$ & $\begin{array}{c}\text { Process 3 } \\
\left\{\begin{array}{l}b \rightarrow c \rightarrow l \\
\bar{b} \rightarrow l\end{array}\right.\end{array}$ & $\begin{array}{c}\text { Process 4 } \\
\begin{array}{l}c \rightarrow l \\
\bar{c} \rightarrow l\end{array}\end{array}$ \\
\hline \hline $\begin{array}{l}\text { Neither } Q \text { nor } \bar{Q} \text { quark un- } \\
\text { dergoes mixing. }\end{array}$ & OS & LS & LS & OS \\
\hline $\begin{array}{l}\text { One of } Q \bar{Q} \text { quarks under- } \\
\text { goes mixing. }\end{array}$ & LS & OS & OS & - \\
\hline $\begin{array}{l}\text { Both } Q \text { and } \bar{Q} \text { quarks un- } \\
\text { dergo mixing. }\end{array}$ & OS & LS & LS & - \\
\hline
\end{tabular}

Table 4.2: Charge combinations of $e$ and $\mu$ for each Process. OS (LS) means oppositesign (like-sign).

a lepton via $\bar{c}(c)$ quark.

The production of $c \bar{c}$ can contribute to $e \mu$ events through their semileptonic decay because we do not distinguish between leptons from $b \bar{b}$ and those from $c \bar{c}$. This process is taken into account in our analysis.

If there is no $B^{0} \bar{B}^{0}$ mixing, the charge signs of leptons are opposite in Process 1 , as mentioned before. The charge signs of leptons are like in Process 2 and 3 , because the charge of a sequentially produced lepton reverses.

Charge combinations of $e$ and $\mu$ for each Process are summarized in Table 4.2. The process of $c \bar{c}$ production is included in the table as Process 4 . If $e \mu$ events consist of Processes $1 \sim 4$, the ratio of the number of LS events $\left(N_{L S}\right)$ to that of OS events $\left(N_{O S}\right)$ is written by

$$
\begin{aligned}
R=\frac{N_{L S}}{N_{O S}} & =\frac{2 \chi(1-\chi) N_{D}+\left[(1-\chi)^{2}+\chi^{2}\right] N_{S}}{\left[(1-\chi)^{2}+\chi^{2}\right] N_{D}+2 \chi(1-\chi) N_{S}+N_{C}} \\
& =\frac{2 \chi(1-\chi)+\left[(1-\chi)^{2}+\chi^{2}\right] f_{s}}{(1-\chi)^{2}+\chi^{2}+2 \chi(1-\chi) f_{s}+f_{c}}
\end{aligned}
$$

where $f_{s}=N_{S} / N_{D}$ and $f_{c}=N_{C} / N_{D}$. The $N_{D}$ and $N_{C}$ are the numbers of events for Process 1 and 4, respectively. The $N_{S}$ is the sum of the number of events for Processes 2 and 3. The probability for each mixing process is summarized in Table 4.3. 


\begin{tabular}{|c|c|}
\hline & Probability \\
\hline $\begin{array}{l}\text { Neither } Q \text { nor } \bar{Q} \text { quark un- } \\
\text { dergoes mixing. }\end{array}$ & $(1-\chi)^{2}$ \\
\hline $\begin{array}{l}\text { One of } Q \bar{Q} \text { quarks under- } \\
\text { goes mixing. }\end{array}$ & $2 \chi(1-\chi)$ \\
\hline $\begin{array}{l}\text { Both } Q \text { and } \bar{Q} \text { quarks un- } \\
\text { dergo mixing. }\end{array}$ & $\chi^{2}$ \\
\hline
\end{tabular}

Table 4.3: Probability for each mixing process.

\begin{tabular}{|c|c|c|c|c|c|c|c|c|}
\hline$\left\{\frac{b}{b}\right.$ & $\begin{array}{l}\rightarrow \\
\rightarrow\end{array}$ & $\begin{array}{l}l+X \\
J / \psi+X\end{array}$ & $\rightarrow$ & $l+$ & $X$ & & & (Process 5) \\
\hline$\left\{\begin{array}{l}b \\
b\end{array}\right.$ & $\begin{array}{l}\rightarrow \\
\rightarrow\end{array}$ & $\begin{array}{l}J / \psi+X \\
l+X\end{array}$ & $\rightarrow$ & $l+$ & $X$ & & & (Process 6) \\
\hline$\left\{\begin{array}{l}b \\
b\end{array}\right.$ & $\begin{array}{l}\rightarrow \\
\rightarrow\end{array}$ & $\begin{array}{l}l+X \\
\bar{c}+W\end{array}$ & $W$ & $\rightarrow$ & $c+\bar{s}$ & $c$ & $\rightarrow \quad l+X$ & (Process 7) \\
\hline$\left\{\frac{b}{b}\right.$ & $\begin{array}{l}\rightarrow \\
\rightarrow\end{array}$ & $\begin{array}{l}c+W \\
l+X\end{array}$ & $W$ & $\rightarrow$ & $\bar{c}+s$ & $\bar{c}$ & $\rightarrow \quad l+X$ & (Process 8$)$ \\
\hline
\end{tabular}

Table 4.4: Other semileptonic decay processes.

In addition to the above four processes, we have considered other processes listed in Table 4.4. In Process $5(6)$, the $b(\vec{b})$ quark decays to a lepton directly and the other $\bar{b}(b)$ quark decays to a $J / \psi$ and then one of the leptons from the $J / \psi \rightarrow l^{+} l^{-}$decay is detected, while the other lepton misses detection. In Process $7(8)$, the $b(\bar{b})$ quark decays to a lepton directly and the other $\bar{b}(b)$ quark to $\bar{c}+c+\bar{s}(c+\bar{c}+s)$ quarks and then the $c(\bar{c})$ quark decays to a lepton. The charge combinations of $e$ and $\mu$ for the other processes are summarized in Tables 4.5 and 4.6. By looking at Eq. (4.2), it is not difficult to understand that inclusion of other $b$-decay processes does not change the shape of this equation. Since the most relevant definition of $f_{s}$ is the ratio of all LS contributions to all OS contributions from $b \bar{b}$ events in the case of no mixing, the numbers of events for Process 5,6,7, and 8 are added to the $N_{D}$, and that for Process 


\begin{tabular}{|l||c|c|}
\cline { 2 - 3 } & $\begin{array}{l}\text { Process 5 } \\
\left\{\begin{array}{l}b \rightarrow l \\
\bar{b} \rightarrow J / \psi \rightarrow l\end{array}\right.\end{array}$ & $\begin{array}{l}\text { Process 6 } \\
\left\{\begin{array}{l}b \rightarrow J / \psi \rightarrow l \\
\bar{b} \rightarrow l\end{array}\right.\end{array}$ \\
\hline \hline $\begin{array}{l}\text { Neither } Q \text { nor } \bar{Q} \text { quark un- } \\
\text { dergoes mixing. }\end{array}$ & OS/LS & OS/LS \\
\hline $\begin{array}{l}\text { One of } Q \bar{Q} \text { quarks under- } \\
\text { goes mixing. }\end{array}$ & LS/OS & LS/OS \\
\hline $\begin{array}{l}\text { Both } Q \text { and } \bar{Q} \text { quarks un- } \\
\text { dergo mixing. }\end{array}$ & OS/LS & OS/LS \\
\hline
\end{tabular}

Table 4.5: Charge combinations of $e$ and $\mu$ for Process 5 and 6. OS (LS) means oppositesign (like-sign).

\begin{tabular}{|l|c|c|}
\cline { 2 - 3 } & $\begin{array}{c}\text { Process } 7 \\
b \rightarrow l \\
\bar{b} \rightarrow c \rightarrow l\end{array}$ & $\begin{array}{c}\text { Process } 8 \\
\left\{\begin{array}{c}b \rightarrow \bar{c} \rightarrow l \\
\bar{b} \rightarrow l\end{array}\right.\end{array}$ \\
\hline \hline $\begin{array}{l}\text { Neither } Q \text { nor } \bar{Q} \text { quark un- } \\
\text { dergoes mixing. }\end{array}$ & OS & OS \\
\hline $\begin{array}{l}\text { One of } Q \bar{Q} \text { quarks under- } \\
\text { goes mixing. }\end{array}$ & LS & LS \\
\hline $\begin{array}{l}\text { Both } Q \text { and } \bar{Q} \text { quarks un- } \\
\text { dergo mixing. }\end{array}$ & OS & OS \\
\hline
\end{tabular}

Table 4.6: Charge combinations of $e$ and $\mu$ for Process 7 and 8. OS (LS) means oppositesign (like-sign). 
5 and 6 are added to the $N_{S}$. Contributions from the other decay processes, therefore, only change the value of $f_{s}$. The $b$-decay processes listed in Tables 4.1 and 4.4 account for most of the $e \mu$ events from $b \bar{b}$ production considered in this analysis. The effect of the other decay processes is relatively small.

Since the observed $e \mu$ events contain fake lepton events, the $R$ is written in terms of the observed numbers of LS and OS e $\mu$ events, $N^{e x p}(L S)$ and $N^{e x p}(O S)$, by

$$
R=\frac{N^{e x p}(L S)\left(1-F_{e \mu}(L S)\right)}{N^{e x p}(O S)\left(1-F_{e \mu}(O S)\right)}
$$

where $F_{e \mu}(L S)$ and $F_{e \mu}(O S)$ are the fake lepton fractions in the LS and OS samples. Fake leptons are caused by lepton mis-identification, photon conversion electrons, and muons from hadron decays in flight.

To determine $\chi$, we need to know $F_{e \mu}(O S), F_{e \mu}(L S), f_{s}$ and $f_{c}$ in addition to $N^{e x p}(O S)$ and $N^{\exp }(L S)$. The studies of these quantities were carried out by using real data as much as possible, with minimum use of Monte Carlo's. The $F_{\varepsilon \mu}$ 's were obtained by studying the fake fraction for each lepton. For muons it was done by using $J / \psi \rightarrow \mu^{+} \mu^{-}$decays, minimum bias data, $K_{s}^{0} \rightarrow \pi^{+} \pi^{-}$decays, and $\phi \rightarrow K^{+} K^{-}$decays. Similarly, the study for electrons was done by using $J / \psi \rightarrow e^{+} e^{-}, \gamma$-hadron overlapped events, and Monte Carlo generated charged pions.

We used Monte Carlo programs to estimate the $f_{s}$. Direct quark pair productions were studied with ISAJET [41] + CLEOMC [42, 43]. Gluon splitting process was also taken into account by using ISALEP [44]+ CLEOMC. The ISAJET is a Monte Carlo program which simulates $p-p$ and $\bar{p}-p$ interactions at high energies. It is based on perturbative QCD plus phenomenological models for parton and beam jet fragmentation. The QFL is a Monte Carlo program which simulates bottom and charm particles decays. The ISALEP is a special version of ISAJET which is modified to generate higher order heavy quark events with high- $P_{T}$ leptons effectively. Both generated events were passed through the QFL detector simulation package [45]. The CLEOMC is a detector simulation program for CDF detector. Since the philosophy of the package is to param- 
eterize detector response rather than derive the response from first principles, the QFL Simulation package can provide a fast and reliable detector simulation for high statistics physics studies and acceptance calculations. We first considered processes listed in Table 4.1, and took into account of other processes separately as a correction.

A semileptonic decay of $b$ and $c$ quarks produces a jet associated with a lepton. The transverse momentum of the lepton to the jet axis, $P_{T}^{\text {rel }}$, defined in Fig. 4.1 is useful to estimate the fraction of the leptons from $c$ quarks to those from $b$ quarks because the mass difference between the quarks causes different $P_{T}^{\text {rel }}$-distributions. We derived the $f_{c}$ by fitting a sum of the $P_{T}^{r e l}$-distributions for different processes to an observed $P_{T}^{r e l}$ distribution.

\subsection{Event selection}

\subsubsection{Muon selection}

Muon candidates were selected by requiring the presence of a CTC track associated with a stub in muon chambers. The muon stub was reconstructed with the central muon chambers (CMU and CMP) and the muon momentum was derived from curvature of the CTC track. The energy deposit in calorimeter towers which a muon candidate traverses was required to correspond to a minimum ionizing particle. Muon identification was performed by applying the following cuts.

\section{Track Quality Cuts}

In order to make sure that the track of a muon candidate comes from a hard collision interaction and is not misreconstructed in the CTC, a set of track quality cuts is imposed as follows:

- A track must be reconstructed in 3 dimension.

- $\left|D_{0}\right|<0.5 \mathrm{~cm}$. 
- $\left|Z_{\text {event }}-Z_{0}\right|<5.0 \mathrm{~cm}$

- number of hits in CTC chamber $>50$

where $D_{0}$ is a radial distance of the track at the closest point to the beam axis, or the impact parameter. $Z_{\text {event }}$ is the $z$ position of the event vertex and $Z_{0}$ is the $z$ position of the track at the closest point to the beam axis. The efficiency of these requirements for charged tracks should be quite high when the charged tracks are produced from $\bar{p} p$ collisions. Fig. 4.2, Fig. 4.3 and Fig. 4.4 show distributions of these parameters for muon candidates in $J / \psi$ events and for non-interacting-punchthrough candidates in a jet sample. The $J / \psi$ events are required to have two muon candidates and the invariant mass of them in a region $3<M_{\mu \mu}<3.15 \mathrm{GeV}$. The jet sample consists of the events triggered by the pre-scaled jet trigger with an $E_{T}$ threshold of $20 \mathrm{GeV}$. Since this sample is dominated by ordinary QCD jet events, most of the muon candidates in the sample can be considered as fake muons. To select non-interacting-punchthrough candidates, they are required to have a stub only in the CMU chamber even in the fiducial area of CMU and CMP chambers. We note that the position matching cuts described below are already imposed on the events shown in Fig. 4.2, Fig. 4.3 and Fig. 4.4. The cut values employed are indicated by arrows in the figures.

\section{Position matching cuts}

Muon selection requires that both CMU and CMP chambers have stubs. The $\triangle X(C M U)$ and $\triangle X(C M P)$ are defined position differences in $(r-\phi)$ space between the reconstructed CTC track and the reconstructed stub in CMU and CMP chamber. Fiducial cuts are applied to avoid cracks between the CMU chambers and regions uncovered with the CMP chambers. This fiducial area is well covered by the calorimeters. Figures 4.5 and 4.6 show $\triangle X(C M U)$ and $\Delta X(C M P)$ distributions for muon candidates in the $J / \psi$ events and for non-interacting-punchthrough candidates in the jet sample.

The $\Delta X(C M U)$ and $\Delta X(C M P)$ distributions for muons can be explained by effects of multiple scattering in the material which muons traverse before reaching in the each 
muon chamber. Until muons reach the CMU chamber, they pass through material of 66 radiation lengths. The rms spread of $\phi$ will be

$$
\Delta \phi_{\text {multiple scattering }}(C M U)[\mathrm{rad}]=\frac{0.128}{P_{T}[\mathrm{GeV} / \mathrm{c}]} .
$$

Since there is $60 \mathrm{~cm}$ steel ( 34 radiation lengths) between CMU and CMP chambers, the rms spread of $\phi$ in the CMP will be

$$
\Delta \phi_{\text {multiple scattering }}(C M P)[\mathrm{rad}]=\frac{0.158}{P_{T}[\mathrm{GeV} / \mathrm{c}]} .
$$

Since the distance from the solenoid coil to the CMU is $203 \mathrm{~cm}$, one standard deviation of $\Delta X$ at $\mathrm{CMU}$ is $5 \mathrm{~cm}$ at $P_{T}=3 \mathrm{GeV} / \mathrm{c}$. For a typical distance of the CMP chambers from the coil of $400 \mathrm{~cm}$ ( the distance change by the location of the CMP), one standard deviation of $\triangle X$ at the CMP is $12 \mathrm{~cm}$ at $P_{T}=3 \mathrm{GeV} / \mathrm{c}$.

The $\chi^{2}(C M U)$ and $\chi^{2}(C M P)$ are a fit $\chi^{2}$ of CTC and chambers track. The $\chi^{2}(C M U)$ and $\chi^{2}(C M P)$ distribution for muon candidates in the $J / \psi$ events and the jet sample are shown in Figs. 4.7 and 4.8.

The position matching cuts used are:

- $\Delta X(C M U)<7 \mathrm{~cm}$

- $\Delta X(C M P)<30 \mathrm{~cm}$

- $\chi^{2}(C M U)<10$

- $\chi^{2}(C M P)<30$

\section{Minimum ionization cuts}

The $E M_{m u o n}$ and $H A D_{m u o n}$ are the electromagnetic and hadronic energy of the calorimeter tower which a muon traverses. Figures 4.9 and 4.10 show the $E M_{\text {muon }}$ and $H A D_{\text {muon }}$ distribution for muons in the $J / \psi$ events and the jet sample. The energy deposit by 
muons in the EM and HAD calorimeters was measured in a test beam to be EMmuon $0.3 \mathrm{GeV}$ and $\mathrm{HAD}$ muon $\sim 2 \mathrm{GeV}$, respectively. The distribution of the sum of the $E M_{m u o n}$ and $H A D_{m u o n}$ is shown in Fig. 4.11. We use the following minimum ionizing particle cuts:

- $E M_{\text {muon }}<2 \mathrm{GeV}$

- $H A D_{m u o n}<4 \mathrm{GeV}$

- $E M_{\text {muon }}+H A D_{\text {muon }}>0.1 \mathrm{GeV}$

\section{Summary of the central muon selection}

The central muon selection is summarized as follows:

1. Track quality cuts.

2. Position matching cuts.

3. Minimum ionization cuts.

4. $P_{T}>3 \mathrm{GeV} / \mathrm{c}$

The cut values used in the selection are listed in Table 4.7. However, the muons selected by the above cuts, still include fake muons, which are mainly due to non-interactingpunchthrough of charged hadrons and decay-in-flight muons from hadrons. We will estimate the fraction of fake muons in Section 5.1.

\subsubsection{Electron selection}

Electron candidates are selected primarily by requiring the large energy deposit in the EM calorimeter and the presence of an associated track in the CTC. 


\section{Energy fraction cut}

A ratio of hadronic energy to electromagnetic energy in a calorimeter cluster, $H A D / E M$, is used to discriminate charged hadrons, which have more energy deposited in the hadronic calorimeter than electrons. Figure 4.12 shows the $H A D / E M$ distributions for $J / \psi \rightarrow e e$ events and the jet sample. The $J / \psi \rightarrow e e$ events are required to have two electron candidates and the invariant mass of them in a region $2.8<M_{e e}<3.2 \mathrm{GeV}$. The jet sample consists of the events triggered by the pre-scaled jet trigger with an $E_{T}$ threshold of $20 \mathrm{GeV}$. The EM clusters in the jet sample are used as fake electrons. We require that

- $H A D / E M<0.04$

$\mathbf{E} / \mathbf{P}$

One of the sources which mimic the electron signature in the detector is a charged hadron overlapping with photon. This overlap of a photon and a charged hadron looks like an EM cluster associated with the track of the charged hadron. A ratio of transverse energy to transverse momentum, $E / P$, is used to distinguish an electron from this overlap. The $E / P$ is not necessarily one in the overlap due to its accidental characteristics, while in the case of electrons it should be around one. Therefore, the $E / P$ is useful to reject this type of backgrounds. Figure 4.13 shows the $E / P$ distributions for electrons in $J / \psi \rightarrow e e$ events and for the EM clusters in the jet sample. We require that

- $E / P<1.4$

\section{Lateral shower shape, $\chi_{\text {strip }}^{2}$, cut}

Comparing a shower shape along the beam $(Z)$ direction measured with the CES strip to that obtained from test beam electrons, one can check the consistency of the measured shower shape with the expected electron shower shape. We minimize the following function by varying two parameters, $Z$, the $Z$ position of the center of a shower, and $E$, 
the electron energy.

$$
\chi^{2}(Z, E)=\sum_{i-5}^{i+5} \frac{\left(E_{i}^{\text {meas }}-E \times q_{i}^{\text {pred }}(Z)\right)^{2}}{\sigma_{i}^{2}(Z)}
$$

where $E_{i}^{\text {meas }}$ is the measured energy on the strip channel $i, q_{i}^{\text {pred }}$ is a normalized energy distribution on channel $i$ predicted from $50 \mathrm{GeV} / \mathrm{c}$ test beam electrons, and $\sigma_{i}$ denotes an energy fluctuation of a single-channel response. In the summation of $i$, neighboring eleven channels are examined corresponding to about $15 \mathrm{~cm}$ which is sufficient to contain a full electron shower. Based on $10 \mathrm{GeV} / \mathrm{c}$ electron test beam data [46], the response fluctuation $\sigma_{i}$ for each channel is parameterized as

$$
\sigma_{i}^{2}(Z)=0.026^{2}+0.096^{2} q_{i}^{p r e d}(Z)
$$

Using the shower center position obtained above, $\chi_{\text {strip }}^{2}$ is defined as

$$
\chi_{\text {strip }}^{2}=\frac{1}{4}\left(\frac{E_{C E M}}{10}\right)^{0.747} \sum_{i=5}^{i+5} \frac{\left(q_{i}^{\text {meas }}-q_{i}^{\text {pred }}\left(Z_{C E S}\right)\right)^{2}}{\sigma_{i}^{2}\left(Z_{C E S}\right)}
$$

where $E_{C E M}$ is the energy measured by the central EM calorimeter, $q_{i}^{\text {meas }}$ is the measured value of the normalized energy distribution on channel $i$. The energy obtained by minimizing function (4.6) is not used because the energy resolution of the strip chamber measurement is worse (20\% and $30 \%$ for $50 \mathrm{GeV} / \mathrm{c}$ and $10 \mathrm{GeV} / \mathrm{c}$ electrons, respectively) than the one measured by the CEM. An energy dependence factor of $E^{0.747}$ is introduced to compensate for the energy dependence of the $\chi_{\text {strip }}^{2}$, which comes from the fact that the CES is located at a fixed depth in the CEM and it therefore see a different age of the longitudinal shower development depending on the electron energy. The parameter was determined from test beam electrons of various energies from $10 \mathrm{GeV}$ to $200 \mathrm{GeV}$. Figure 4.14 shows $\chi_{\text {strip }}^{2}$ distributions for the electrons from $J / \psi \rightarrow$ ee and for the EM cluster in the jet sample.

The $\chi_{\text {strip }}^{2}$ is useful to discriminate an EM cluster of an electron against an EM 
cluster in which more than one particle are contained. Photons from $\pi^{0} \rightarrow \gamma \gamma$ decays have a minimum opening distance $d(\mathrm{~cm})$ at a radius $R(\mathrm{~cm})$ from the beam line that is related to the $\pi^{0}$ momentum as

$$
d=\frac{2 R M_{\pi}}{P_{T}(\pi)} \quad\left(P_{T} \text { in } \mathrm{GeV} / \mathrm{c}\right)
$$

where $P_{T}(\pi)(\mathrm{GeV} / \mathrm{c})$ and $\left.M_{\pi}=0.135 \mathrm{GeV} / \mathrm{c}^{2}\right)$ are the transverse momentum and the mass of $\pi^{0}$. At the CES $(R=184 \mathrm{~cm})$, we have $d \sim 50 / P_{T}$. Since number of strips for calculation of $\chi_{s}^{2}$ trip is eleven $(\sim 15 \mathrm{~cm})$, two photons with $d<7.5 \mathrm{~cm}$ and two photons from $\pi^{0}$ of $P_{T}(\pi)$ above $\sim 7 \mathrm{GeV} / \mathrm{c}$ will be contained in a same window for calculation of $\chi_{s}^{2}$ trip. The resulting $\chi_{\text {strip }}^{2}$ will have a large value due to the existence of an additional shower peak in the cluster. A typical CES shower has $\sim 99 \%$ of the total CES energy in $\pm 2.5 \mathrm{~cm}$ around the shower center. Therefore the presence of two photons will be removed by a large value of $\chi_{\text {strip }}^{2}$ up to $P_{T}\left(\pi^{0}\right) \sim 20 \mathrm{GeV} / \mathrm{c}$. Above this momentum, the two photons get closer to make it difficult to recognize two showers in the CES.

This variable is also used to discriminate electrons against charged pions which interact and deposit mast of the energy in the CEM, since their shower shapes are different from those for electrons. We require that

- $\chi_{\text {strip }}^{2}<10$

\section{Position matching cut}

The position difference in $(r-\phi)$ plane between the position measured by the CES and that of a CTC track extrapolated to the CES, $\triangle X(C E S)$, is defined by

$$
\Delta X(C E S)=R \Delta \phi \quad(R=184 \mathrm{~cm})
$$

where $\Delta \phi$ is the azimuthal difference between the $\phi$ position which is optimized in a similar way as in the $\chi_{\text {strip }}^{2}$ estimation and that of the extrapolated CTC-track at the CES, and $R$ is the radius of the CES from the beam line. Figure 4.15 shows $\triangle X(C E S)$ 
distributions for electrons from the $J / \psi \rightarrow e e$ events and for EM clusters of the jet sample.

The position difference along the beam direction, $\triangle Z(C E S)$, is estimated from a position measurement in the CES and the extrapolated CTC-track position at the CES. The distributions of $\triangle Z(C E S)$ for electrons from the $J / \psi \rightarrow e e$ events and for EM clusters of the jet sample are shown in Fig. 4.16. We require that

- $\Delta X(C E S)<1.5 \mathrm{~cm}$

- $\triangle Z(C E S)<2.5 \mathrm{~cm}$

This position matching is useful to remove the overlap of a photon with a charged hadron where an accidentally associated track of the charged hadron is expected to have a worse position matching than an electron track. In Section 5.1, we will use EM cluster events which have a worse position matching as overlap events of a photon with a charged hadron.

\section{Lateral shower sharing, $L_{\text {shr }}$, cut}

This variable, $L_{\mathrm{shr}}$, describes a lateral sharing of the EM shower energy among the CEM towers in a cluster and is defined by

$$
L_{\mathrm{shr}}=0.14 \sum_{i} \frac{E_{i}^{\text {meas }}-E_{i}^{\text {pred }}}{\sqrt{(\Delta E)^{2}+\left(\Delta E_{i}^{\text {pred }}\right)^{2}}}
$$

where $E_{i}^{\text {meas }}$ is the energy deposit in tower $i, E_{i}^{\text {pred }}$ is the energy expected in tower $i, \Delta E$ is the uncertainty in the energy measurement with the CEM, and $\Delta E_{i}^{\text {pred }}$ is the error associated with $E_{i}^{\text {pred }}$. The sum runs over the two towers in $\eta$ direction adjacent to the seed tower. The expected tower energy $E_{i}^{\text {pred }}$ was determined from test beam electron data as a function of the seed tower energy and the direction of the CES shower center relative to the event vertex. The error $\Delta E_{i}^{p r e d}$ was determined by propagating an error of the shower center measurement in the CES to the predicted energy $E_{i}^{\text {pred }}$. Figure 4.17 
shows $L_{\text {shr }}$ distributions for the electrons from $J / \psi \rightarrow e e$ events and for the EM clusters in the jet sample. We require that

$$
\text { - } L_{\mathrm{shr}}<0.2
$$

\section{Photon conversion removal}

Electrons from photon conversions have to be removed from the electron sample. A photon conversion can occur before entering the VTX, in the beam pipe material, in the layers of SVX and in the inner wall of the VTX. This type of photon conversions is called an inner photon conversion. Also a photon can convert after exiting the VTX, in the outer wall of the VTX and in the inner wall of the CTC. This is called an outer photon conversion. In both cases, the electron tracks can be detected in the CTC, but the outer photon conversions do not leave tracks in the VTX. A ratio of the number of VTX wire hits to the number of wires which are expected to be fired by a particle, VTX hit occupancy, can be used to discriminate charged particles from neutral particles in the VTX. Electrons from outer photon conversions have a low value of the VTX hit occupancy. In order to remove electrons from inner photon conversions, an additional oppositely charged CTC track is searched and the minimum invariant mass of them, $m_{e-t r a c k}$, is examined. Two electrons from inner photon conversions are expected to have a low mass. An electron which fails one of the following requirements is removed as a conversion electron,

- VTX hit occupancy $>0.5$

- $m_{\mathrm{e}-\text { track }}>0.2 \mathrm{GeV}$.

Figure 4.18 shows a distribution of conversion points for the conversion electron sample in terms of the radial distance from beam line, $R$. We see peaks around the SVX layers $R \sim 3-7 \mathrm{~cm}$, the VTX inner wall $R \sim 22 \mathrm{~cm}$ and the CTC inner wall $R \sim 28 \mathrm{~cm}$. 


\section{Summary of the central electron selection}

The requirements for the central electrons are summarized as follows:

1. Energy fraction cut.

2. E/P cut.

3. Lateral shower shape cut.

4. Position matching cut.

5. Lateral shower sharing cut.

6. Photon conversion removal.

7. $E_{T}>5 \mathrm{GeV}$

The cut values used in the selection are listed in Table 4.7. The selected electrons still have fake electrons which are mainly charged hadrons, overlapped events with photon, and photon conversion events. We will study the fraction of fake electrons using real data and Monte Carlo data in Section 5.1.

\subsection{3 $b \bar{b}$ event selection}

\section{Event vertex cut}

In order to ensure that events are well covered by the tracking and calorimeter detectors, the $Z$ position of event vertex, $Z_{\text {event }}$, required to be near the nominal event vertex. Figure 4.19 shows the $Z$ position of event vertex for the $J / \psi$ events as an example. We require that

$$
\text { - } Z_{\text {vertex }}<60 \mathrm{~cm}
$$

in order to accept $90 \%$ of hard collision events. 
$\ddot{H}_{T}$ cut

Missing transverse energy, $E_{T}$, is defined by the minus of the sum of transverse energies deposited in calorimeter towers over the range $|\eta|<3.6$,

$$
\ddot{E}_{T} \equiv\left|-\sum_{\text {tower }} \vec{E}_{T}{ }^{\text {tower }}\right|
$$

where $\vec{E}_{T}^{\text {tower }}$ is a two-dimensional vector pointing from the event vertex to the tower centroid. For a tower included in the sum, its energy must be above a given threshold. The threshold is $0.1 \mathrm{GeV}$ in the central electromagnetic and hadronic calorimeters, $0.3 \mathrm{GeV}$ in the plug electromagnetic calorimeters, $0.5 \mathrm{GeV}$ in the plug hadronic and forward electromagnetic calorimeters, and $0.8 \mathrm{GeV}$ in the forward hadronic calorimeters. If there is no large- $E_{T}$ neutrino nor jets escaping the detector, the distribution of the energy deposit in the transverse plane view in the calorimeters is expected to be balanced within jet energy resolutions and the $Z_{T}$ is small.

Since $W+$ fake lepton events followed by $W \rightarrow l \nu$ decays cause background to the $e \mu$ events, the $Z_{T}$ is required not to be large. The following $E_{T}$ cut is imposed:

- $Z_{T}<15 \mathrm{GeV}$

\section{$P_{T}^{\text {rel }}$ and jet axis distance cuts}

For the $e \mu$ events we required each lepton to accompany one jet. The jet axis was determined by an algorithm shown in Fig. 4.21. We first pick up a seed track closes to the lepton within a cone size of $\Delta R=0.8$. Tracks near the seed track are added to form a jet. The cone size in defining the jet is $\Delta R=0.8$. The jet axis is calculated from momenta of tracks in the jet without the lepton momentum. Since each jet must come from different quarks, a distance between the jets axes associated to an electron and a muon in $\eta$ - $\phi$ plane, $\Delta R(J e t(e)-J e t(\mu))$, was defined and was required to be greater than 0.1 .

The distributions of the transverse momentum of the lepton relative to the jet axis, 
$P_{T}^{\text {rel }}$, for $b \bar{b}$ and $c \bar{c}$ Monte Carlo events are shown in Figs. 4.22 and 4.23. The $P_{T}^{\text {rel }}$ is related to a mass of the parent particle. A requirement of the $P_{T}^{\text {rel }}$ to be greater than $1.5 \mathrm{GeV} / \mathrm{c}$ reduces the $c \bar{c}$ background. This $P_{T}^{\text {rel }}$ cut is also expected to be effective in removing Process 2 and 3 . The jet axis distance and $P_{T}^{\text {rel }}$ cut employed are:

- $\Delta R(\operatorname{Jet}(e)-J e t(\mu))>0.1$

- $P_{T}^{\text {rel }}(\mu)>1.5 \mathrm{GeV} / \mathrm{c}$

- $P_{T}^{r e l}(e)>1.5 \mathrm{GeV} / \mathrm{c}$

\section{Aximuthal opening angle cut}

A cut on an azimuthal opening angle $\Delta \phi(e \mu)$ between an electron and a muon removes cascade-decay events in which $b(\bar{b})$ quarks decay semileptonically to $c(\bar{c})$ quarks and successively the $c(\bar{c})$ quarks decay semileptonically,

$$
\begin{aligned}
& b(\bar{b}) \rightarrow c+\mu^{-}+X\left(\bar{c}+\mu^{+}+X\right) \quad \text { or } \quad b(\bar{b}) \rightarrow c+e^{-}+X\left(\bar{c}+e^{+}+X\right) \\
& \hookrightarrow e^{+}+X\left(e^{-}+X\right) \quad \hookrightarrow \mu^{+}+X\left(\mu^{-}+X\right)
\end{aligned}
$$

Figures 4.24 (a) and (b) show Monte Carlo $\Delta \phi(e \mu)$ distributions for these semileptonic cascade decays and for $b \bar{b}$ production followed by Process $1 \sim 3$. Semileptonic cascade decays events are distributed around $\Delta \phi(e \mu) \sim 0$. On the contrary, $b \bar{b}$ direct production events are distributed not at small $\Delta \phi(e \mu)$ but at large $\Delta \phi(e \mu)$. The $b \bar{b}$ production events from gluon splitting are distributed at small $\Delta \phi(e \mu)$ but their contribution to the $b \bar{b}$ events are small. The $\Delta \phi(e \mu)$ distribution for real data is shown in Fig. 4.24 (c). There is a bump at small $\Delta \phi(e \mu)$ for OS events but not for LS. This indicates the bump originates from the semileptonic cascade decays because they produce OS events. In order to remove the semileptonic cascade decay events, the following azimuthal opening angle cut is adopted:

- $\Delta \phi(e \mu)>45^{\circ}$ 


\begin{tabular}{|c|c|}
\hline Muon ID & $\begin{array}{l}\text { muon candidate has a CMU stub and a CMP stub } \\
\text { CMU and CMP fiducial cut } \\
E M<2.0 \mathrm{GeV} \\
H A D<4.0 \mathrm{GeV} \\
E M+H A D>0.1 \mathrm{GeV} \\
P_{T}>3 \mathrm{GeV} / \mathrm{c} \\
\text { number of hits in } \mathrm{CTC}>50 \\
\text { impact parameter }<0.5 \mathrm{~cm} \\
\left|Z_{\text {track }}-Z_{\text {vertex }}\right|<\mathrm{cm} \\
\Delta X(\mathrm{CMU})<10 \mathrm{~cm} \\
\chi^{2}(\mathrm{CTC} / \mathrm{CMU})<10 \\
\Delta X(\mathrm{CMP})<30 \mathrm{~cm} \\
\chi^{2}(\mathrm{CTC} / \mathrm{CMP})<30\end{array}$ \\
\hline Electron ID & $\begin{array}{c}E_{T}>5 \mathrm{GeV} \\
H A D / E M<0.04 \\
L_{\text {share }}<0.2 \\
\text { number of } 3 \mathrm{D} \text { tracks }=1 \\
P_{T}>3 \mathrm{GeV} / \mathrm{c} \\
E_{T} / P_{T}<1.4 \\
\chi^{2}(C E S)<10 \\
\Delta X(C E S)<1.5 \mathrm{~cm} \\
\Delta Z(C E S)<2.5 \mathrm{~cm} \\
\text { VTX hit fraction }>0.5 \\
\text { invariant mass of an } e \text { and each track }>0.2 \mathrm{GeV} / \mathrm{c}^{2}\end{array}$ \\
\hline & $\begin{array}{c}\left|Z_{\text {vertex }}\right|<60 \mathrm{~cm} \\
\mathbb{E}_{T}<15 \mathrm{GeV} \\
\text { each lepton accompanies one jet } \\
P_{T}^{\text {rel }}(e)>1.5 \mathrm{GeV} / \mathrm{c} \\
P_{T}^{\text {rel }}(\mu)>1.5 \mathrm{GeV} / \mathrm{c} \\
\Delta R(J e t(e)-J e t(\mu))>0.1 \\
\Delta \phi(e \mu)>45^{\circ}\end{array}$ \\
\hline
\end{tabular}

Table 4.7: Summary of the $e \mu$ event selection. 


\subsubsection{Results of event selection}

After all the selection cut, we found 1710 opposite-sign and 861 like-sign $e \mu$ events in RUN-Ia data corresponding to $20 \mathrm{pb}^{-1}$. In the like-sign events, $420 e^{+} \mu^{+}$and $441 e^{-} \mu^{-}$ events were found. In the opposite-sign events, $850 e^{+} \mu^{-}$and $860 e^{-} \mu^{+}$events were found. 


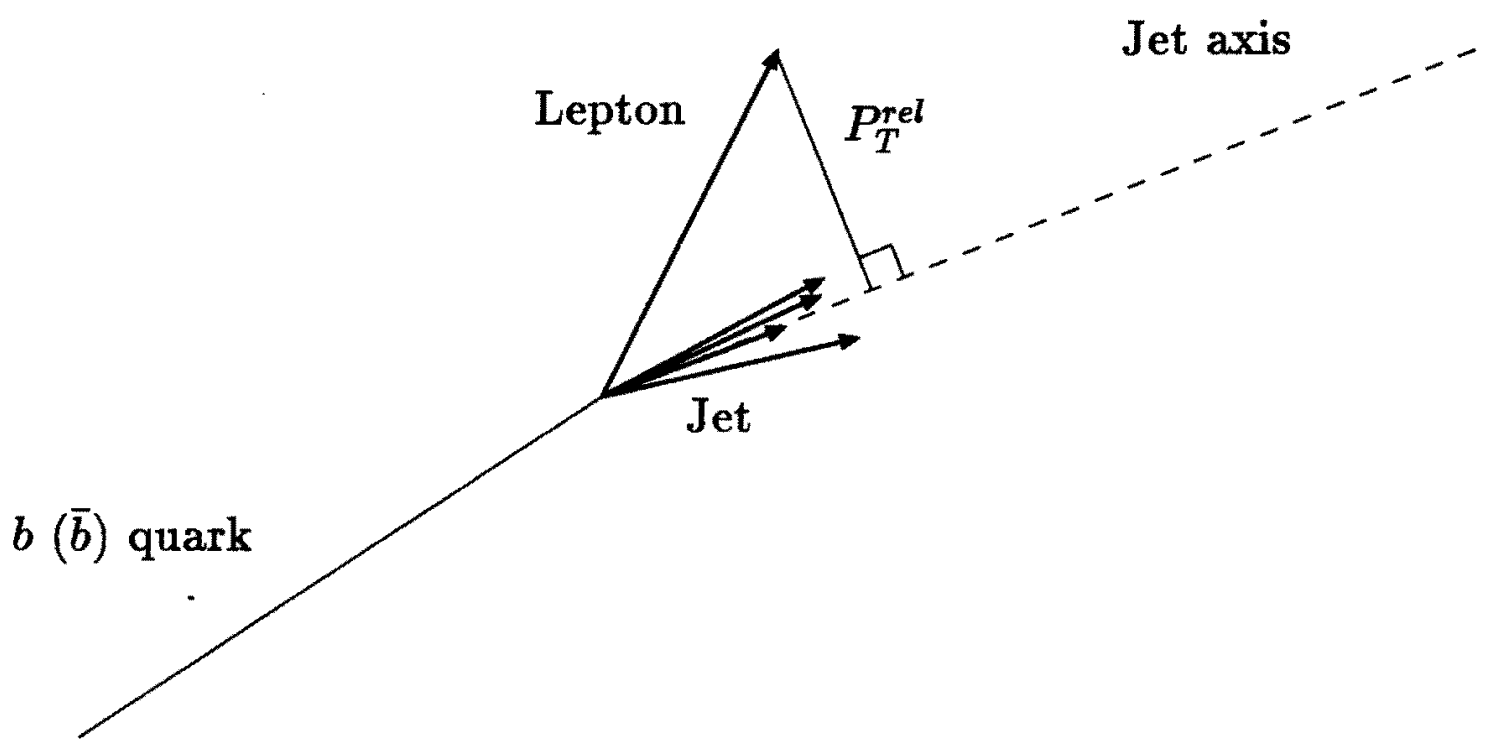

Figure 4.1: Definition of $P_{T}^{\text {rel }}$. 


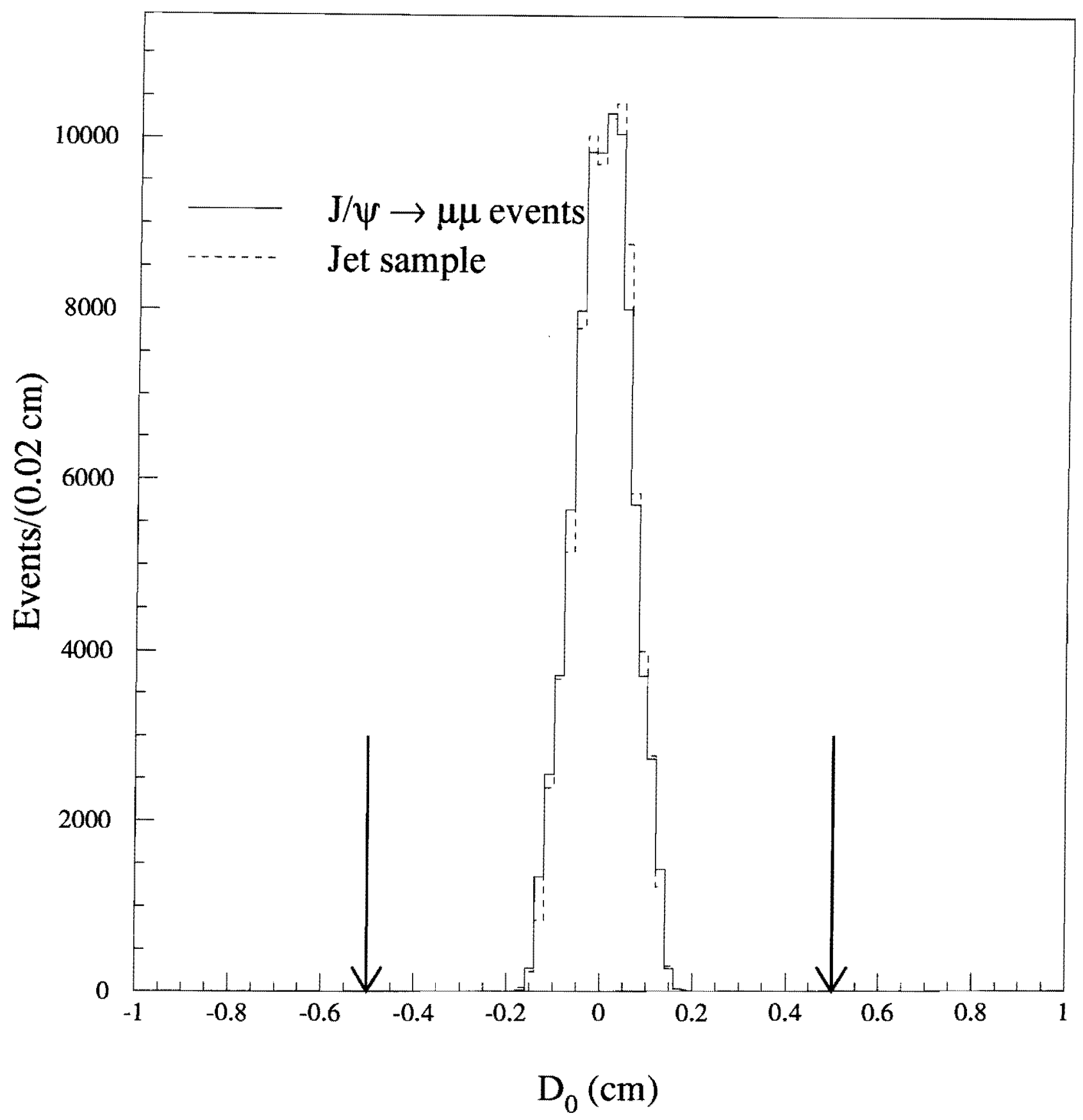

Figure 4.2: The distribution of the impact parameter of muon tracks; $D_{0}$. The distributions for muon candidates in the $J / \psi \rightarrow \mu \mu$ events (solid) and those for the jet sample (dotted) are shown. 


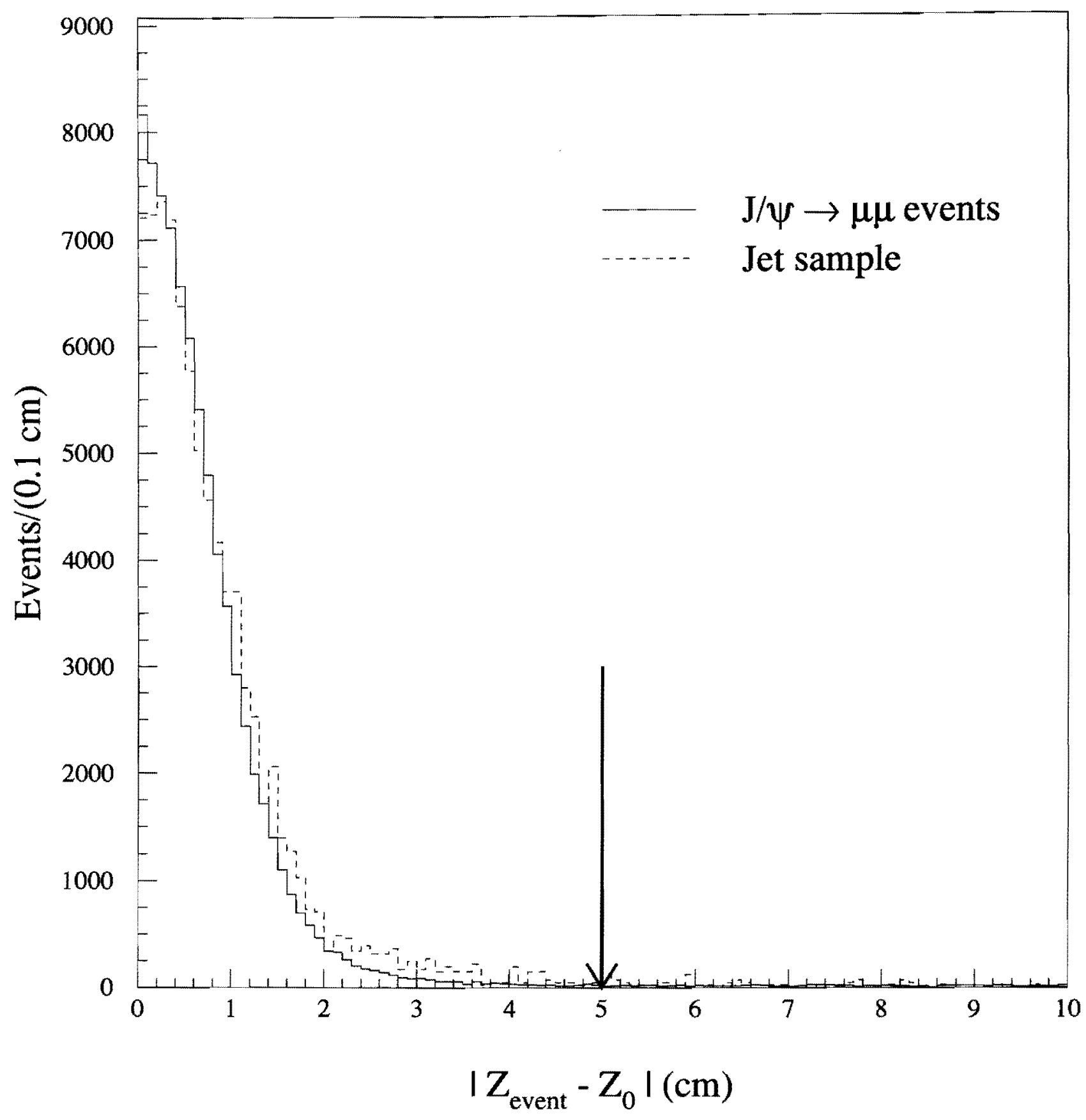

Figure 4.3: The distribution of the difference between the $z$ position of event vertex and the $z$ position of muon tracks; $\left|Z_{\text {event }}-Z_{0}\right|$. The distributions for muon candidates in the $J / \psi \rightarrow \mu \mu$ events (solid) and those for the jet sample (dotted) are shown. 


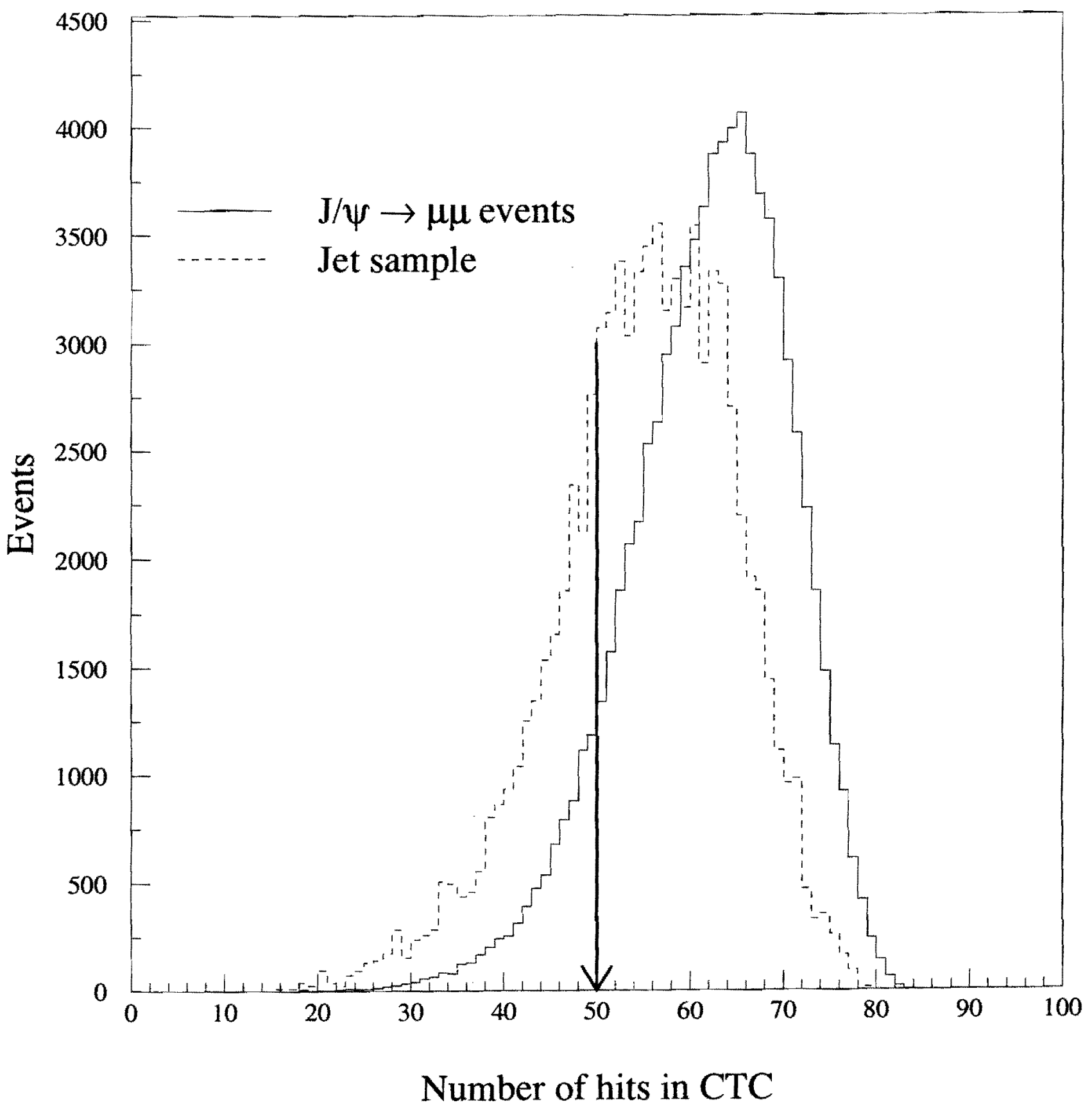

Figure 4.4: The distribution of the number of hits in CTC chamber. The distributions for muon candidates in the $J / \psi \rightarrow \mu \mu$ events (solid) and those for the jet sample (dotted) are shown. 


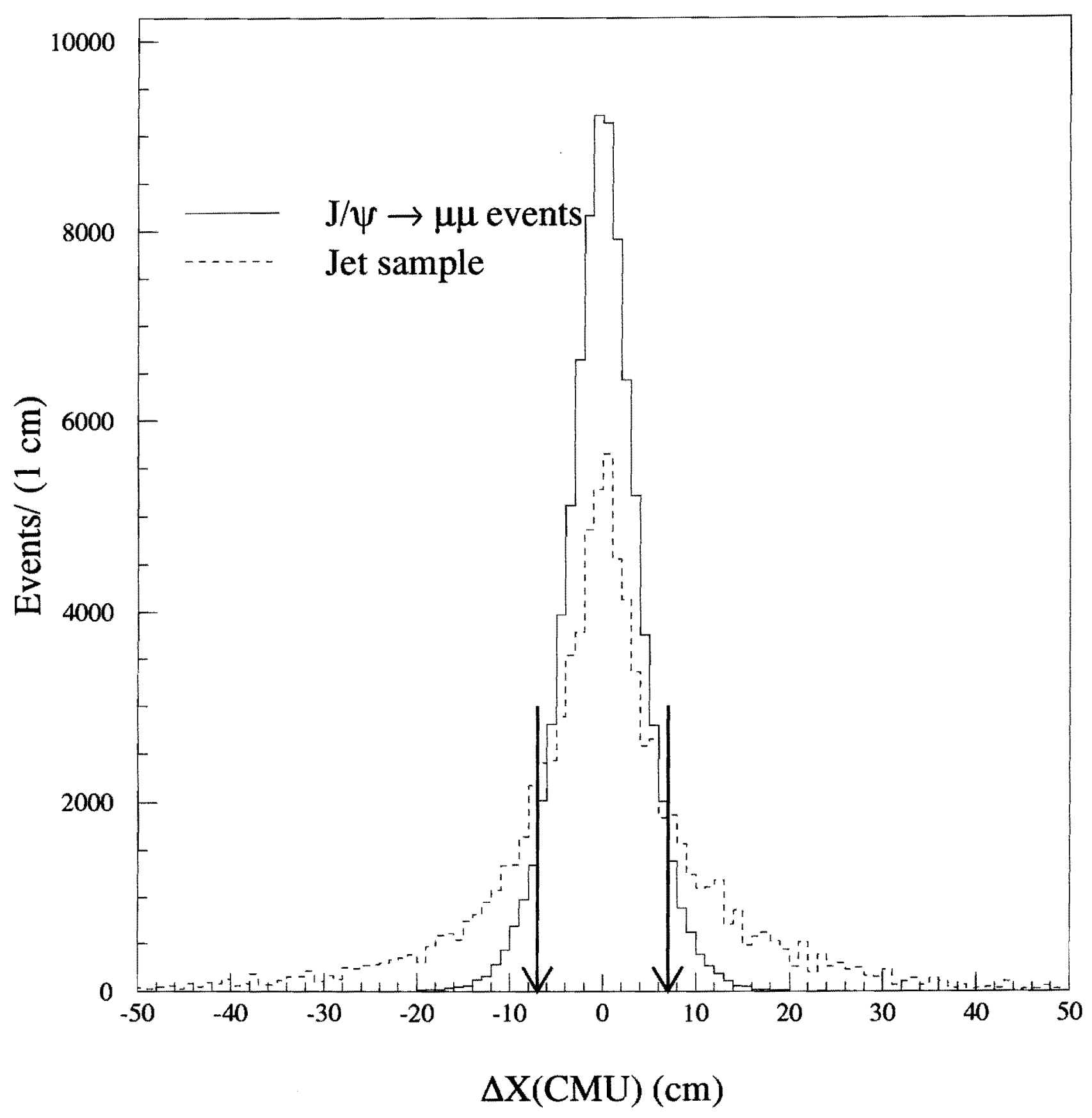

Figure 4.5: The distribution of position matching between a stub in the CMU chambers and an extrapolated CTC track to the CMU; $\triangle X(C M U)$. The distributions for muon candidates in the $J / \psi \rightarrow \mu \mu$ events (solid) and for the jet sample (dotted) are shown. 


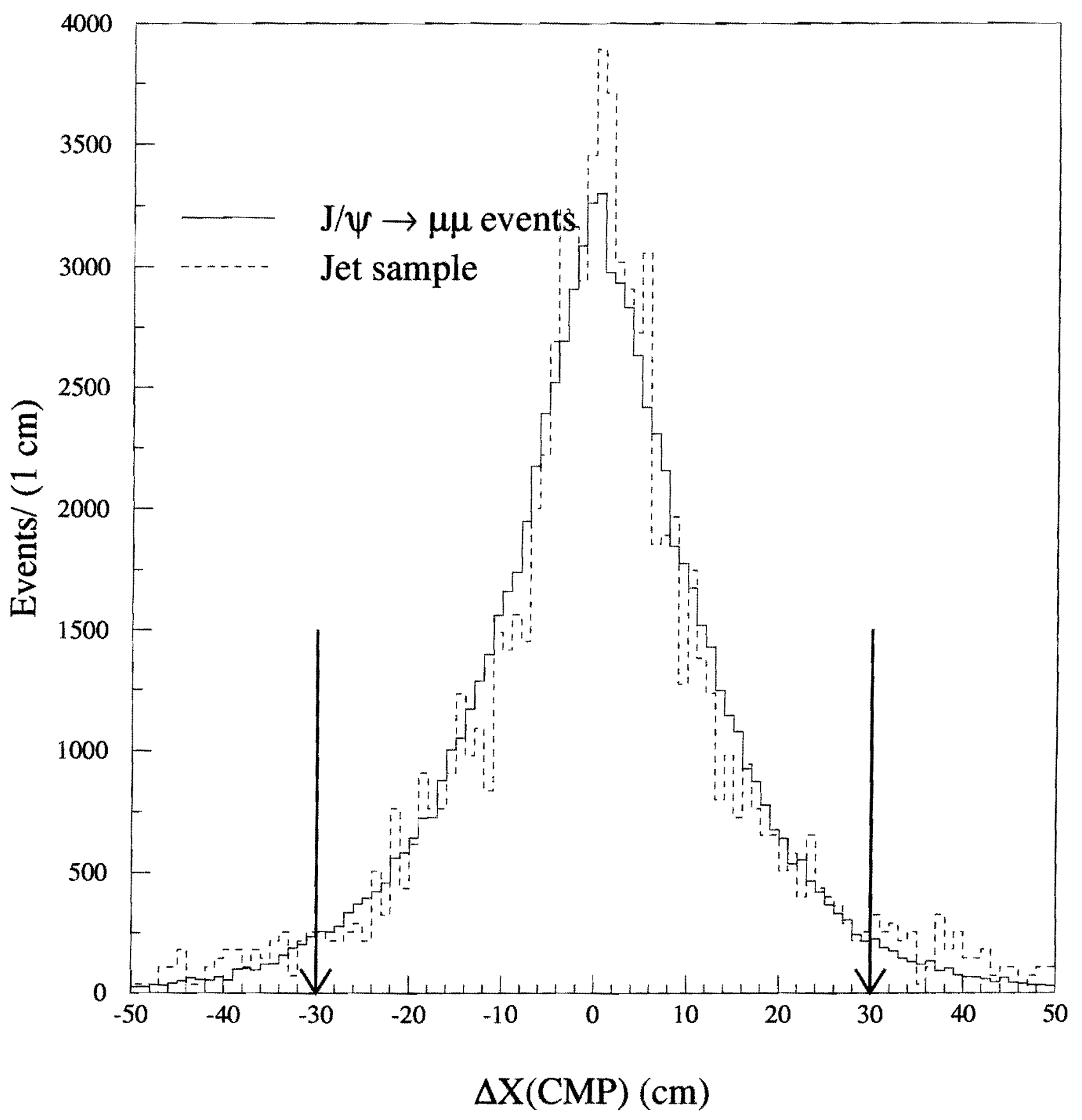

Figure 4.6: The distribution of position matching between a stub in the CMP chambers and an extrapolated CTC track to the CMP; $\triangle X(C M P)$. The distributions for muon candidates in the $J / \psi \rightarrow \mu \mu$ events (solid) and for the jet sample (dotted) are shown. 


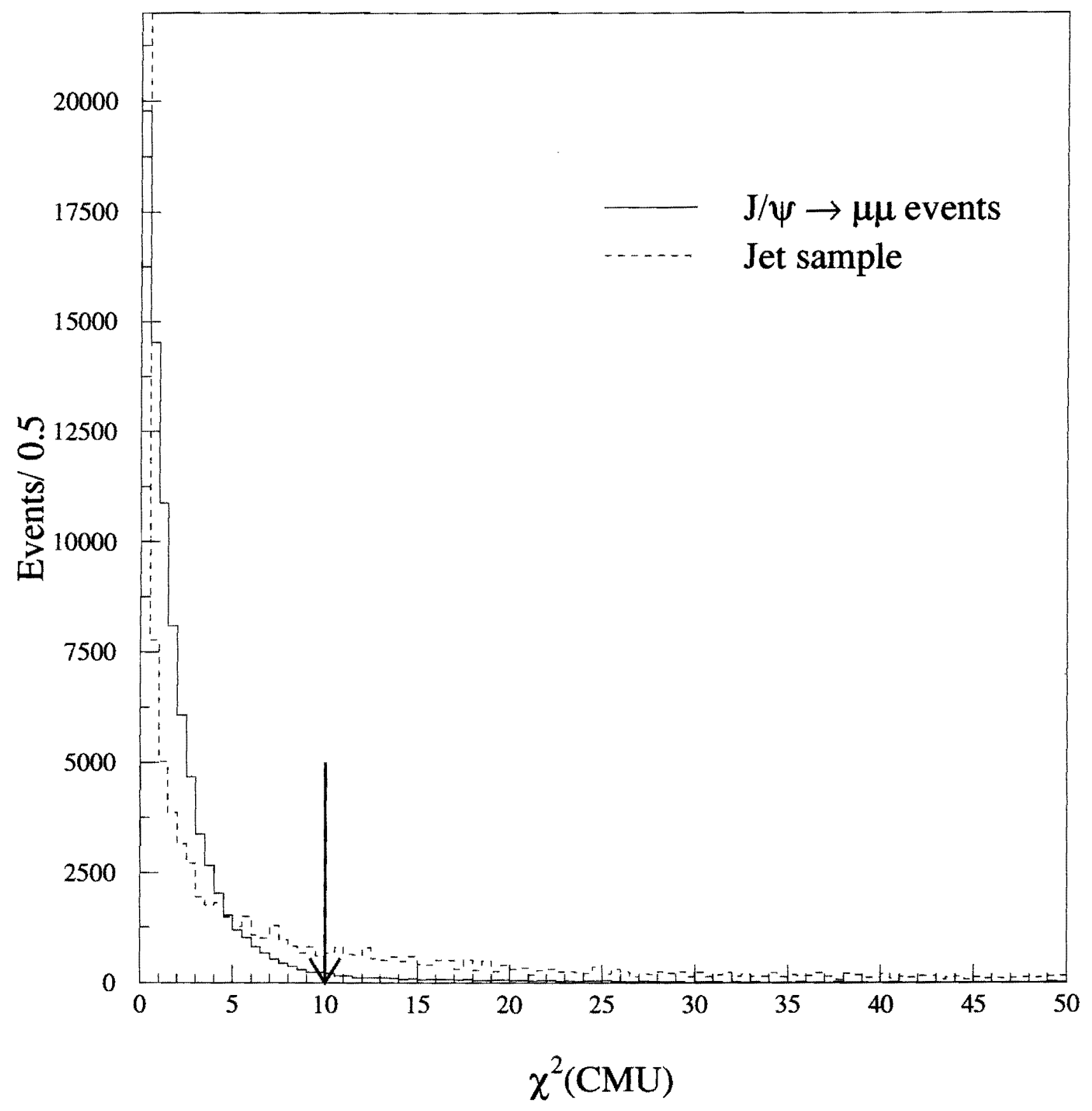

Figure 4.7: The distribution of $\chi^{2}$ for position matching between a stub in the CMU chambers and an extrapolated CTC track to the $\mathrm{CMU} ; \chi^{2}(C M U)$. The distributions for muon candidates in the $J / \psi \rightarrow \mu \mu$ events (solid) and for the jet sample (dotted) are shown. 


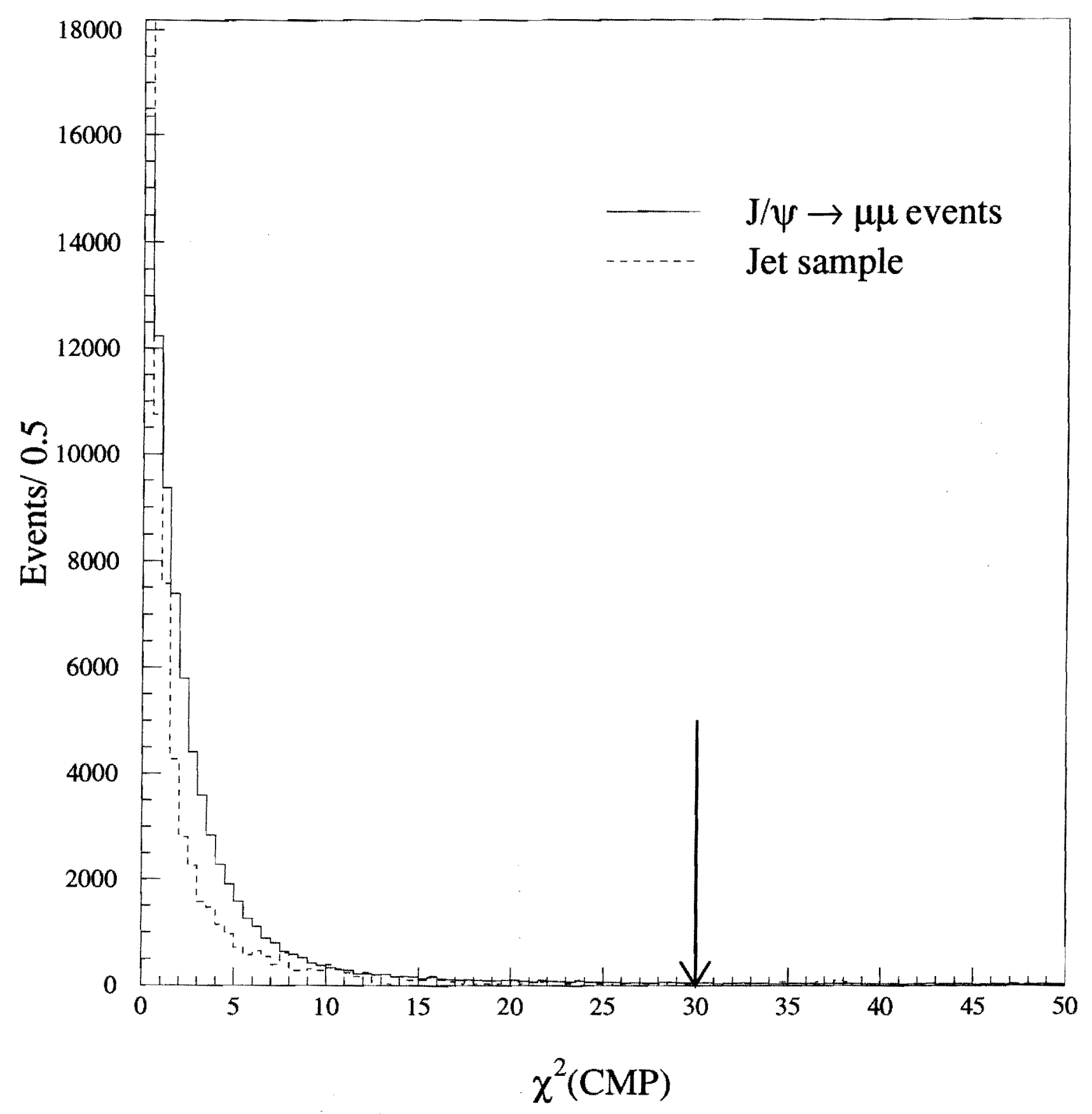

Figure 4.8: The distribution of $\chi^{2}$ for position matching between a stub in the CMP chambers and an extrapolated CTC track to the CMP; $\chi^{2}(C M P)$. The distributions for muon candidates in the $J / \psi \rightarrow \mu \mu$ events (solid) and for the jet sample (dotted) are shown. 


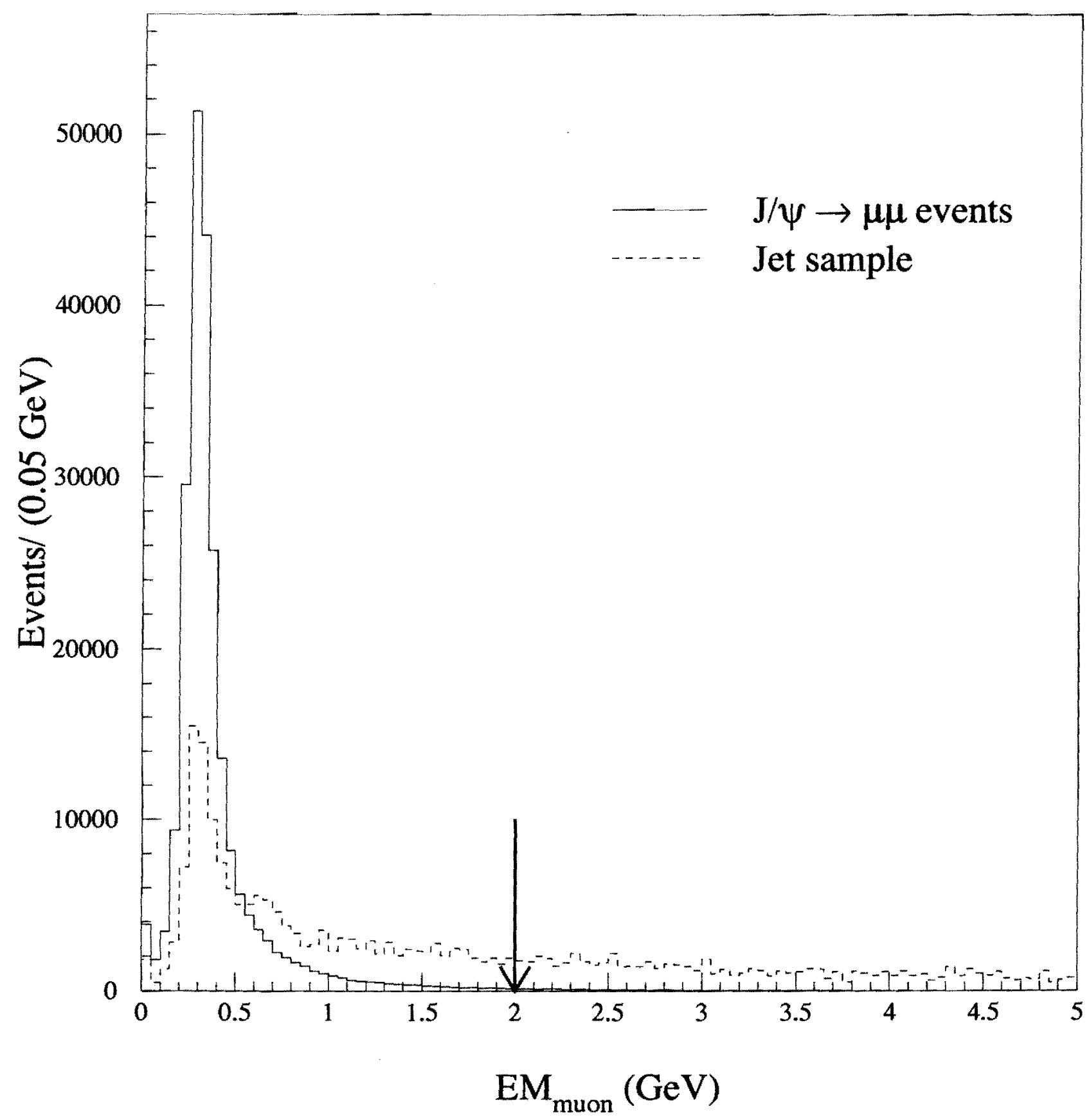

Figure 4.9: The energy of electromagnetic tower which a muon traverses. The distributions from the $J / \psi \rightarrow \mu \mu$ (solid) and the jet sample (dotted) are shown. 


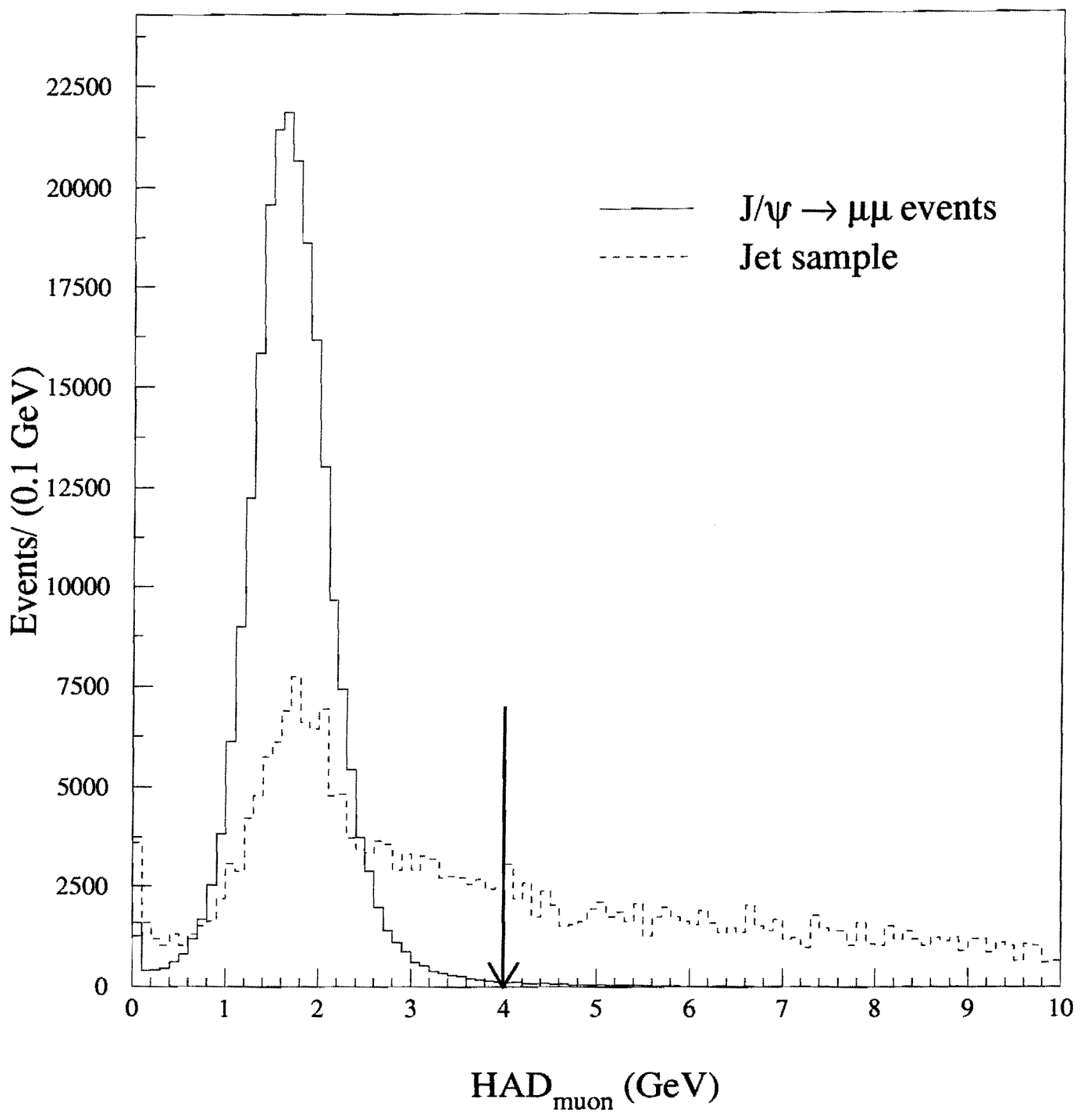

Figure 4.10: The energy of hadronic tower which a muon traverses. The distributions from the $J / \psi \rightarrow \mu \mu$ (solid) and the jet sample (dotted) are shown. 


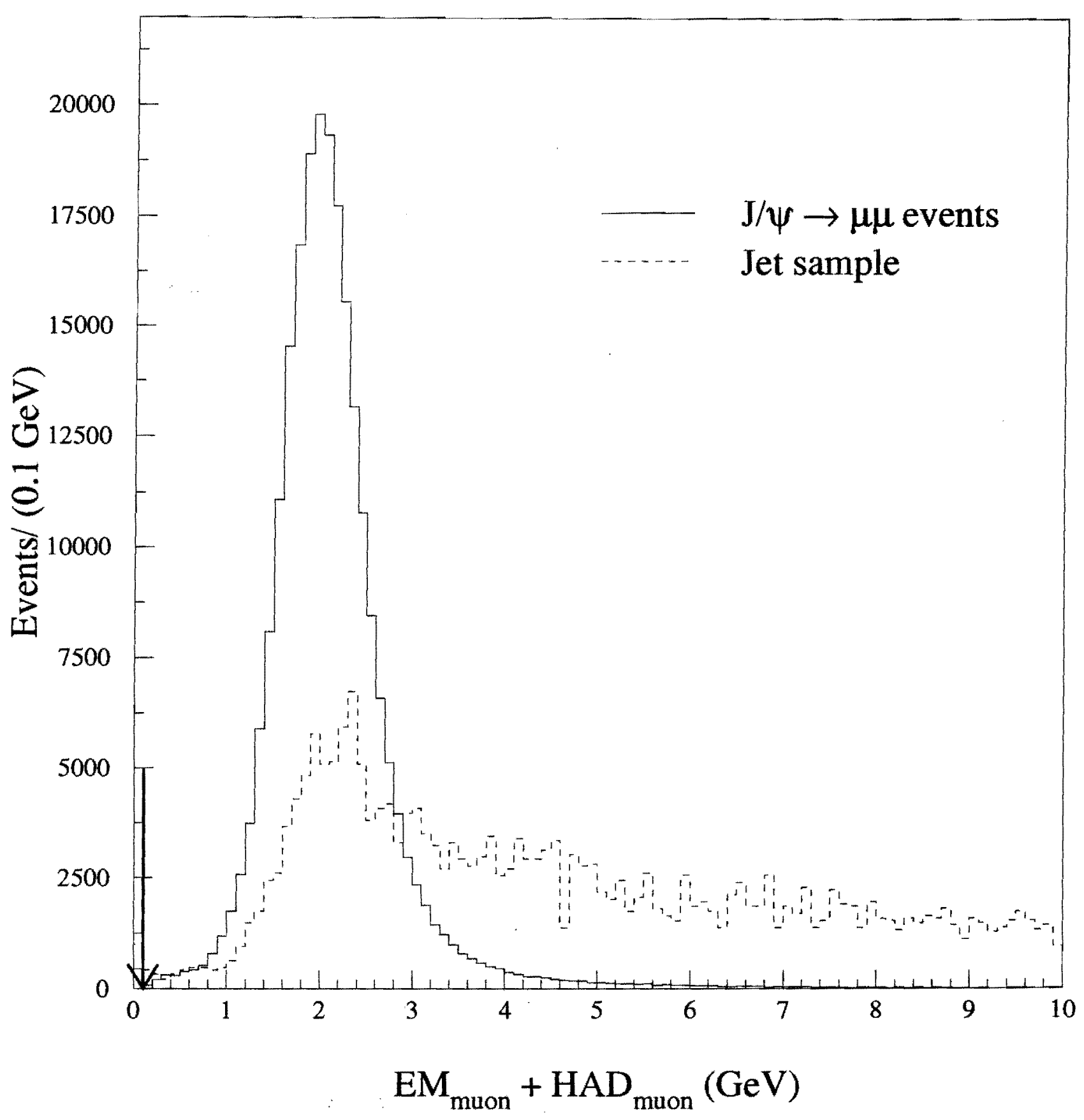

Figure 4.11: The energy sum of electromagnetic and hadronic tower which a muon traverses. The distributions from the $J / \psi \rightarrow \mu \mu$ (solid) and the jet sample (dotted) are shown. 


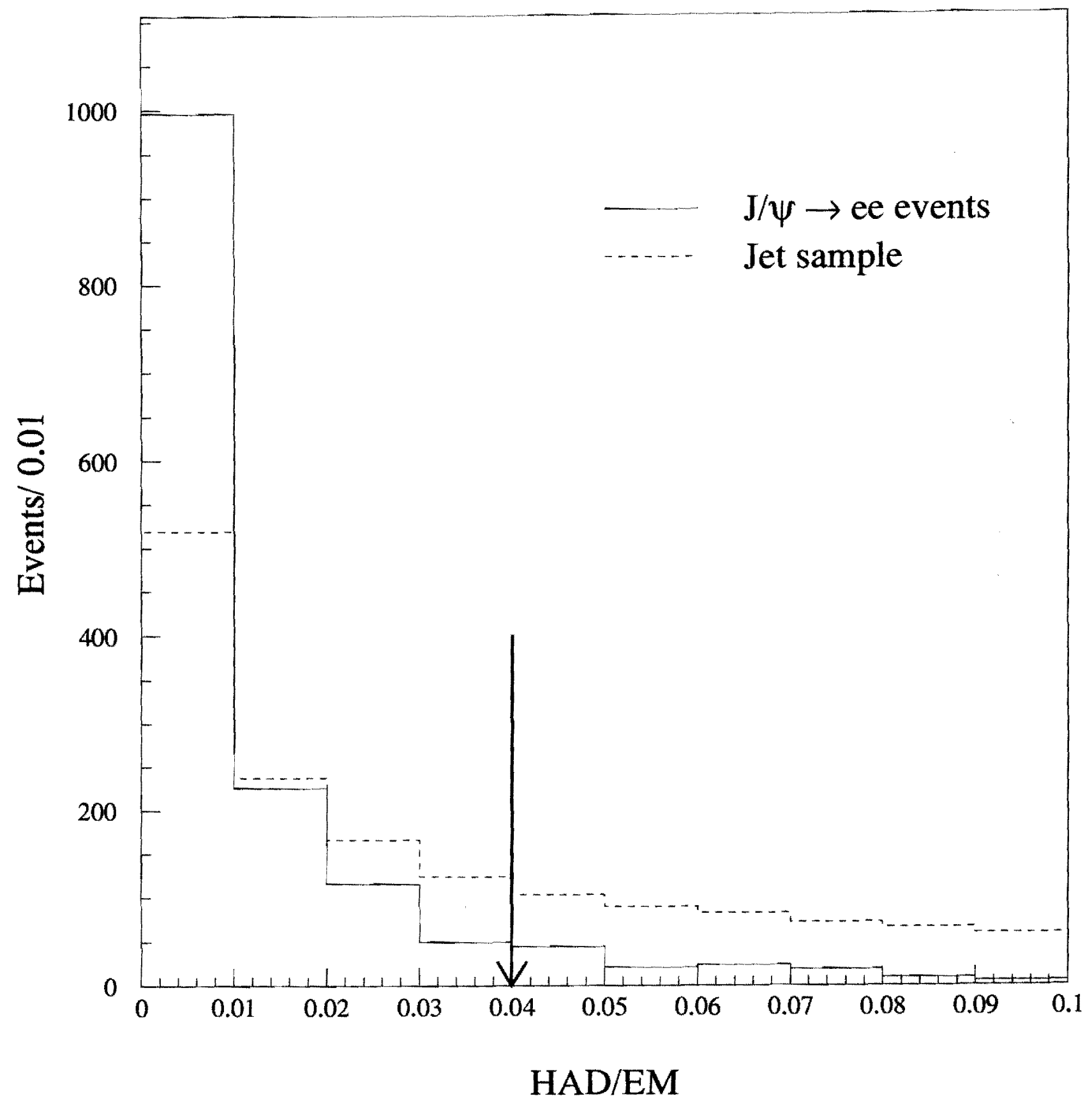

Figure 4.12: The HAD/EM distributions for central electrons in the $J / \psi \rightarrow e e$ (solid) and the jet sample (dotted). 


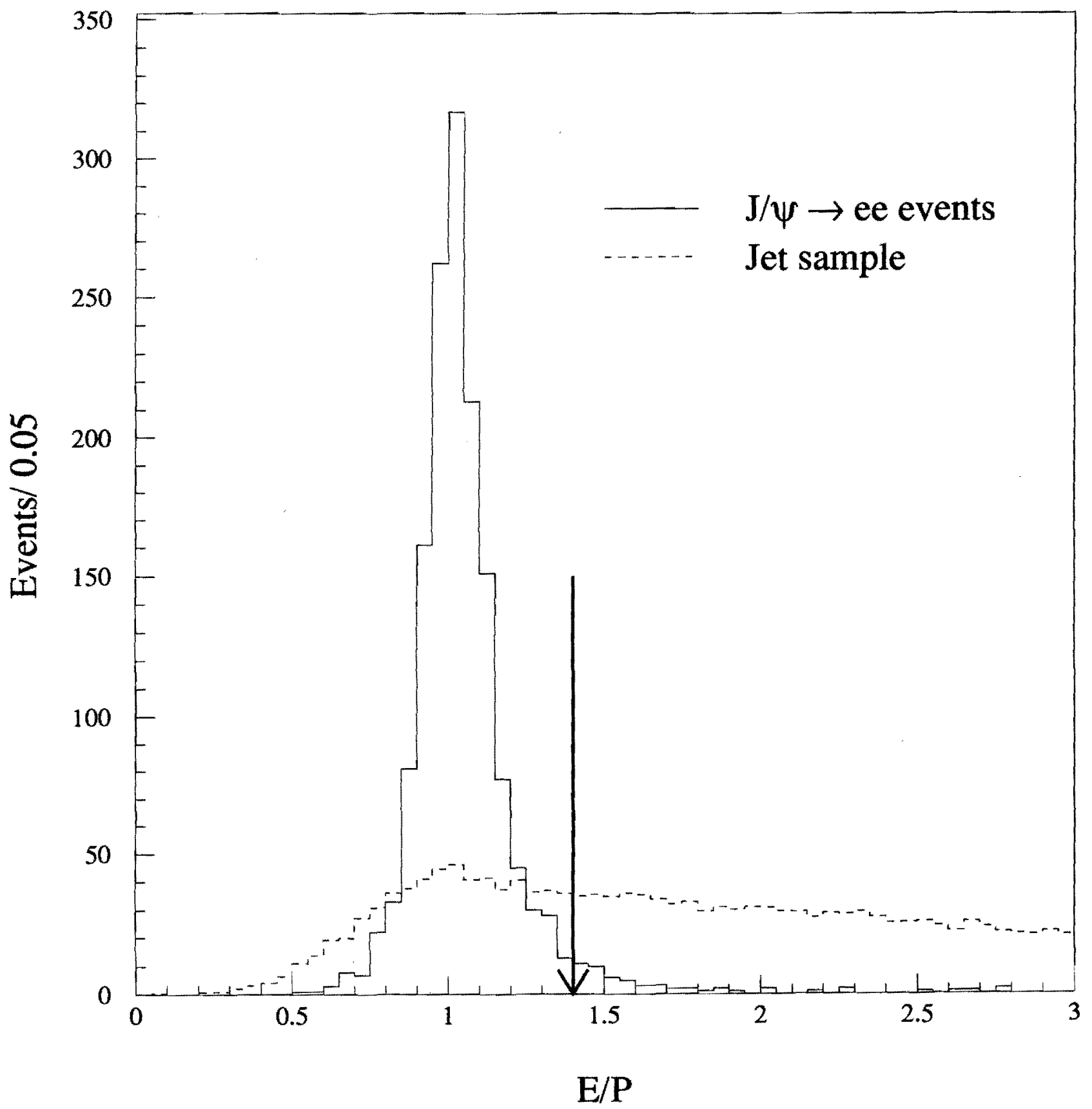

Figure 4.13: The E/P distributions for central electrons in the $J / \psi \rightarrow e e$ (solid) and the jet sample (dotted). 


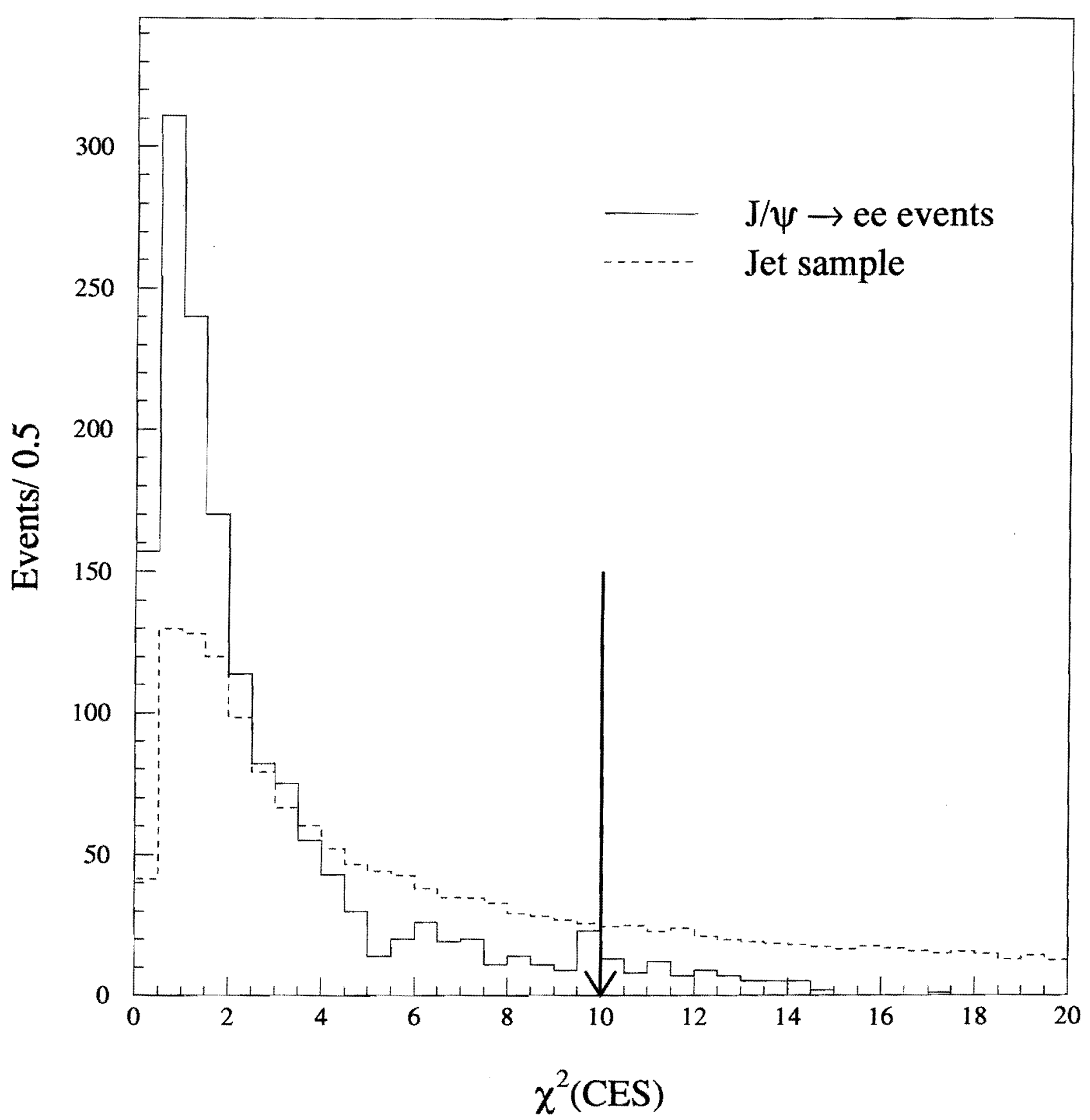

Figure 4.14: The lateral shower shape, $\chi_{\text {strip }}^{2}$, distributions for electrons in the $J / \psi \rightarrow e e$ events (solid) and for EM clusters of the jet sample (dotted). 


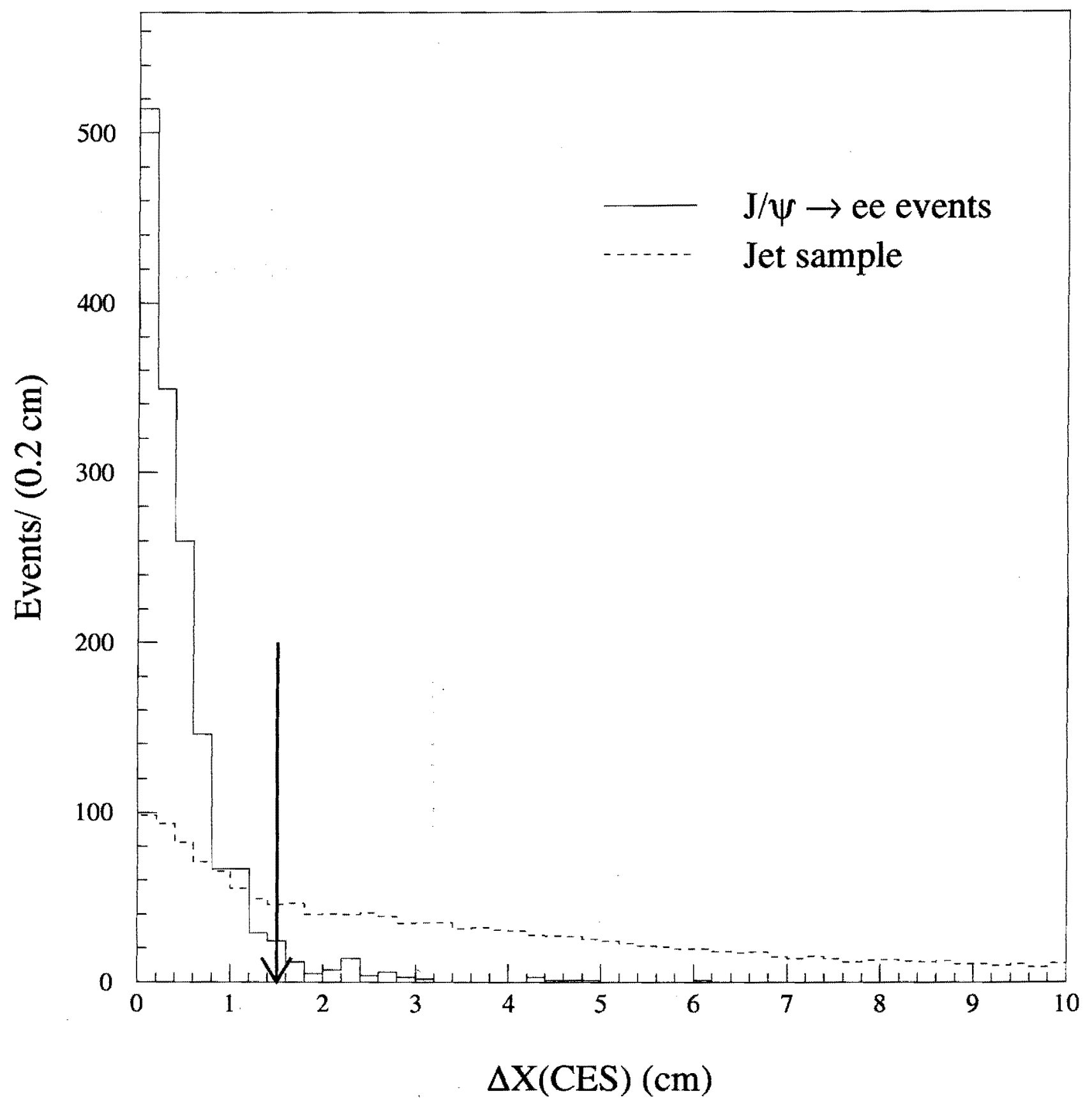

Figure 4.15: The position difference in $(r-\phi)$ plane between the position measured in the CES and that of a track extrapolated to the CES, $\triangle X(C E S)$. The distributions for electrons in the $J / \psi \rightarrow e e$ events (solid) and for EM clusters of the jet sample (dotted). 


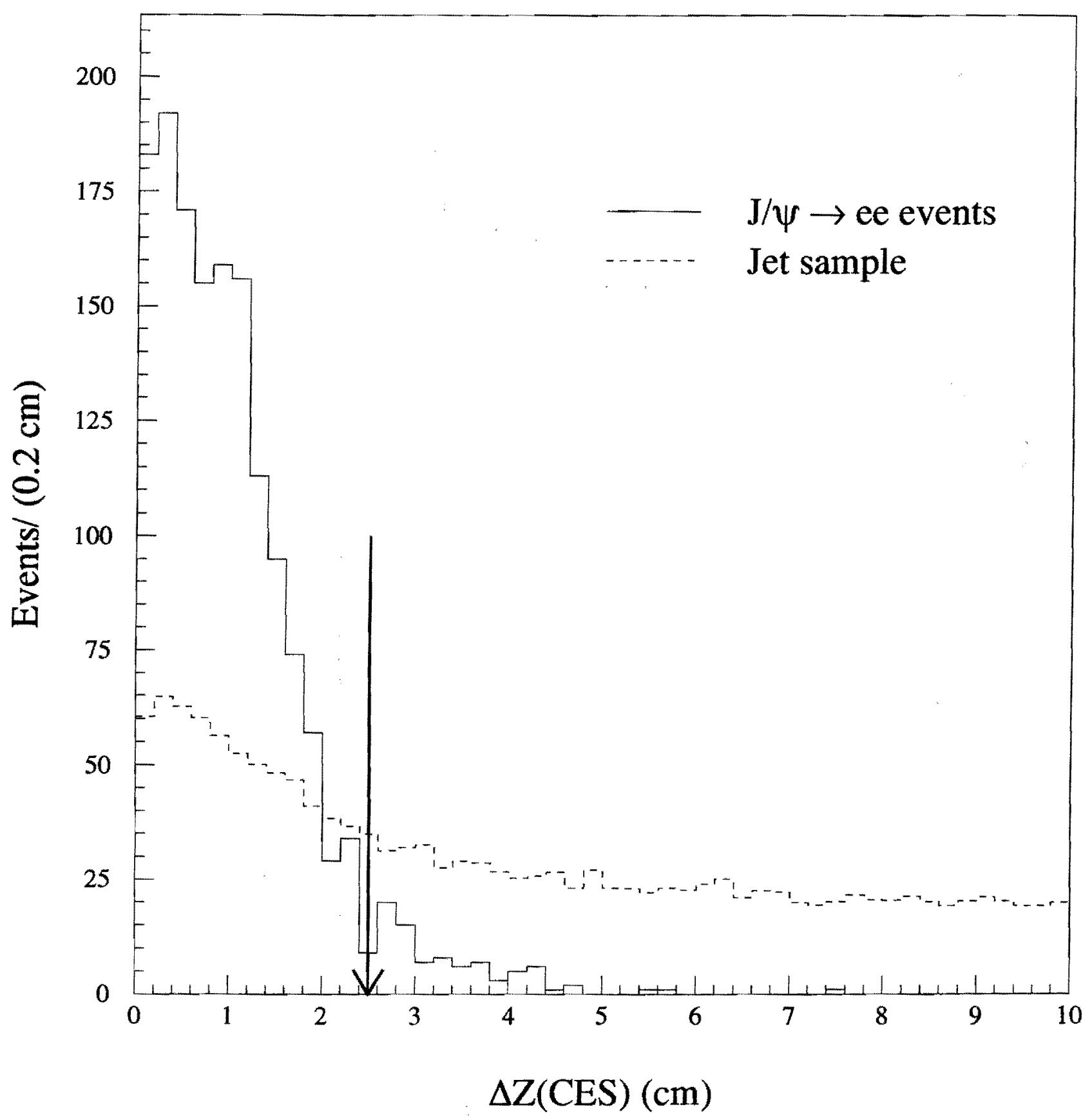

Figure 4.16: The position difference along the beam direction between the $Z$ position measured in the CES and that of a track extrapolated to the CES, $\triangle Z(C E S)$. The distributions for electrons in the $J / \psi \rightarrow e e$ events (solid) and for EM clusters in the jet sample (dotted). 


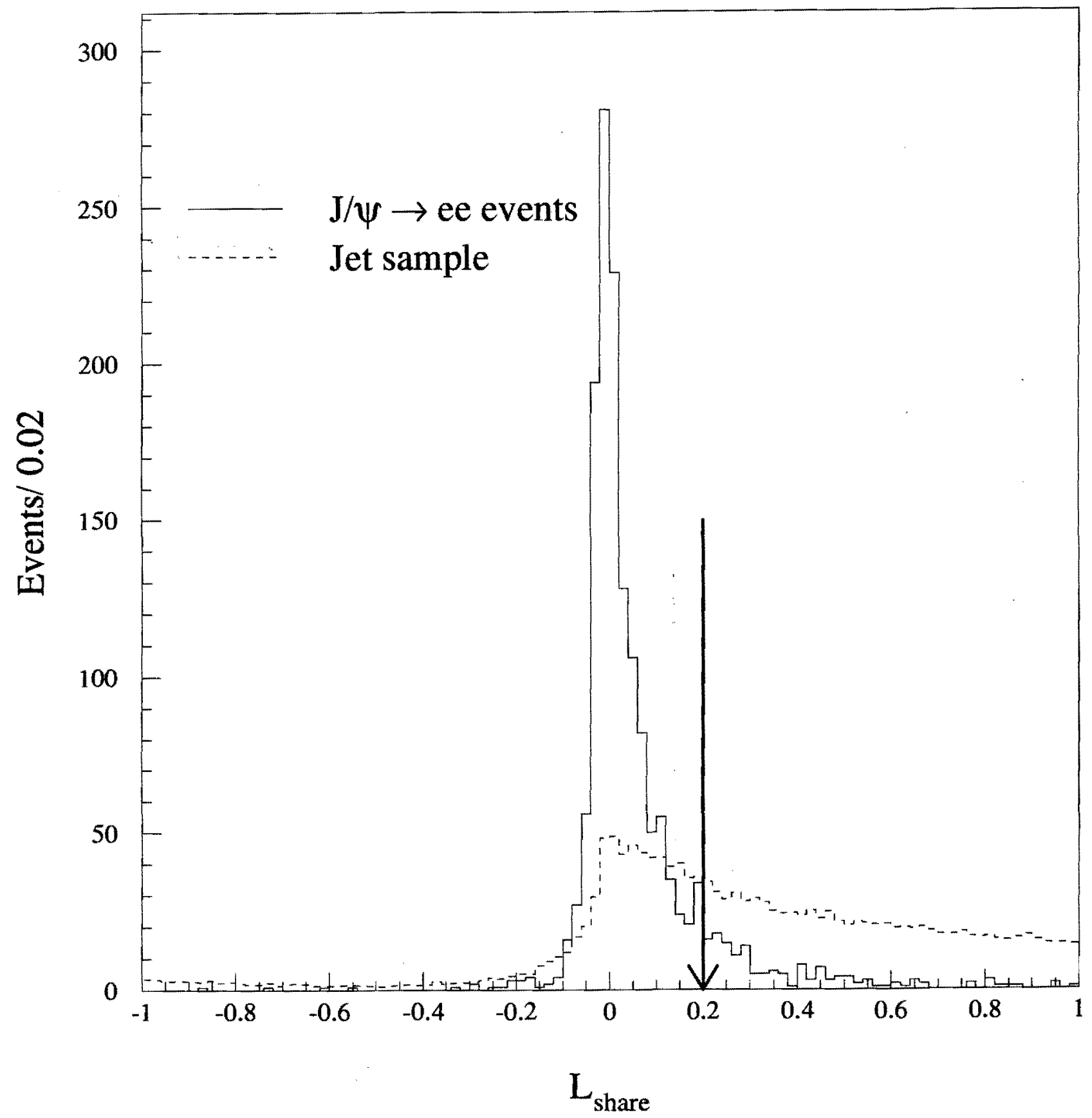

Figure 4.17: The lateral shower sharing, $L_{\mathrm{shr}}$, for electrons in the $J / \psi \rightarrow e e$ (solid) and for EM clusters in the jet sample (dotted). 


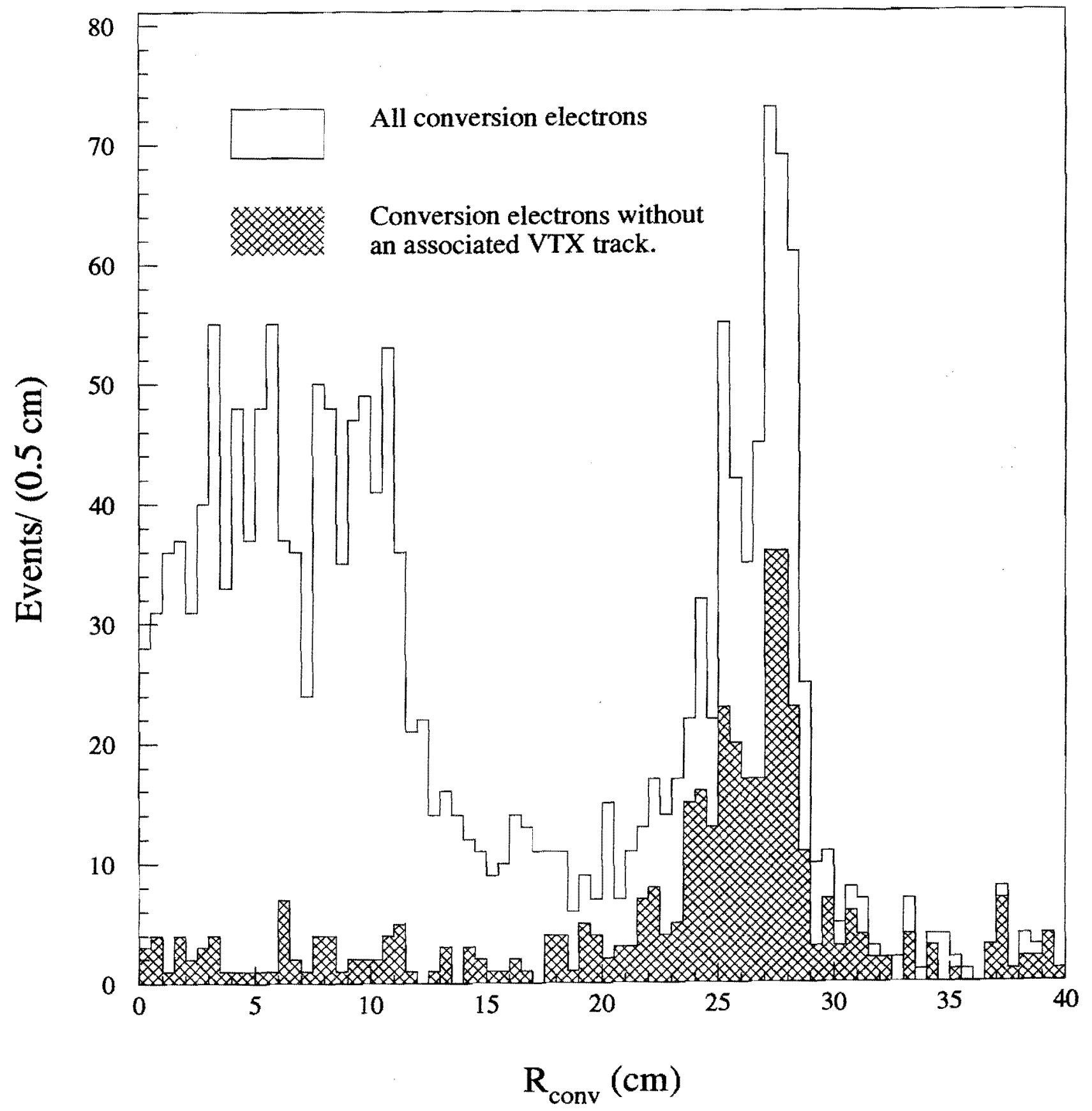

Figure 4.18: The radial distance of the conversion points from the central conversion electron sample. 


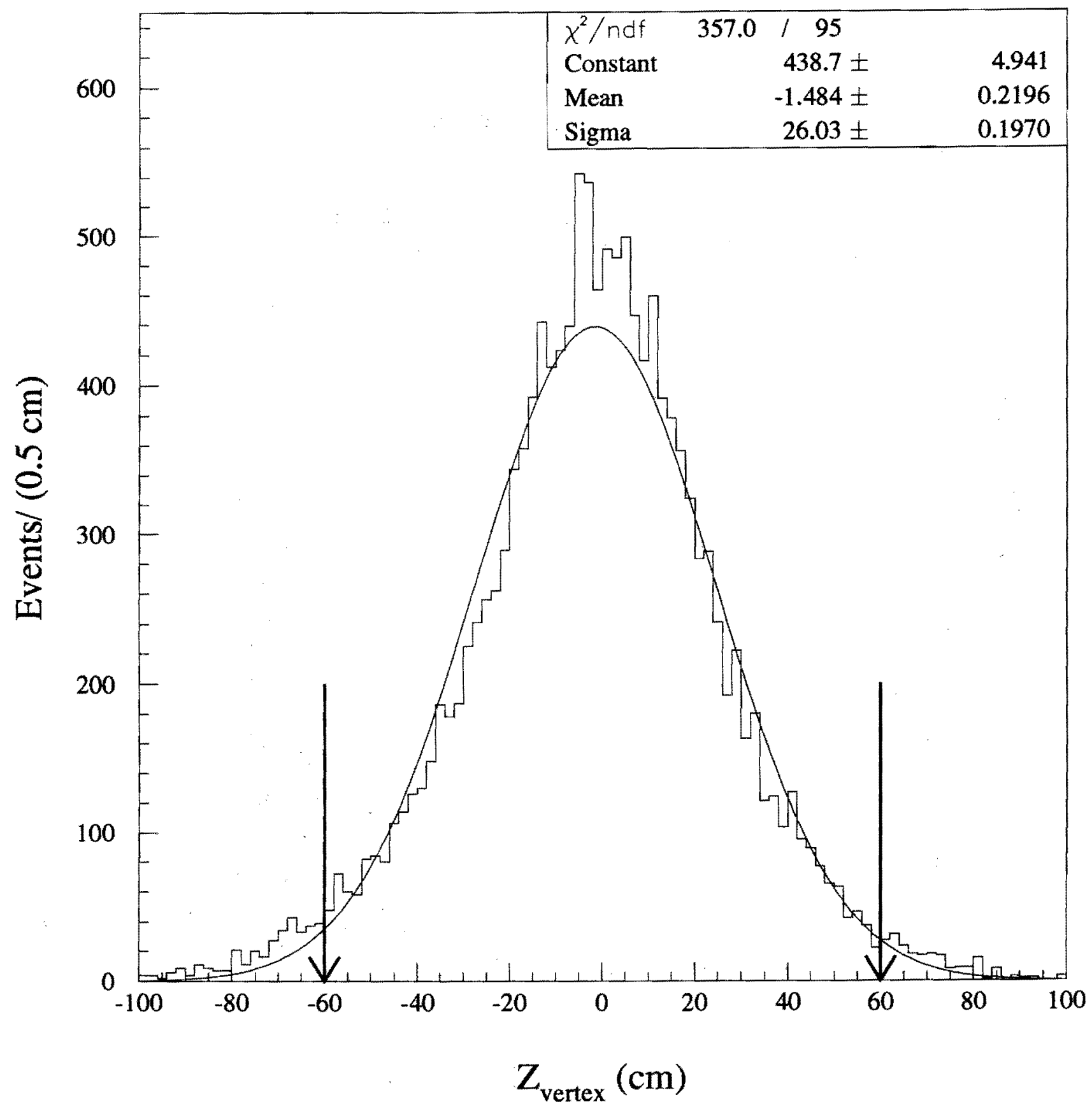

Figure 4.19: The $z$ position of event vertex for $J / \psi \rightarrow \mu \mu$ events. The measured distribution and the fitted gaussian function are shown. 


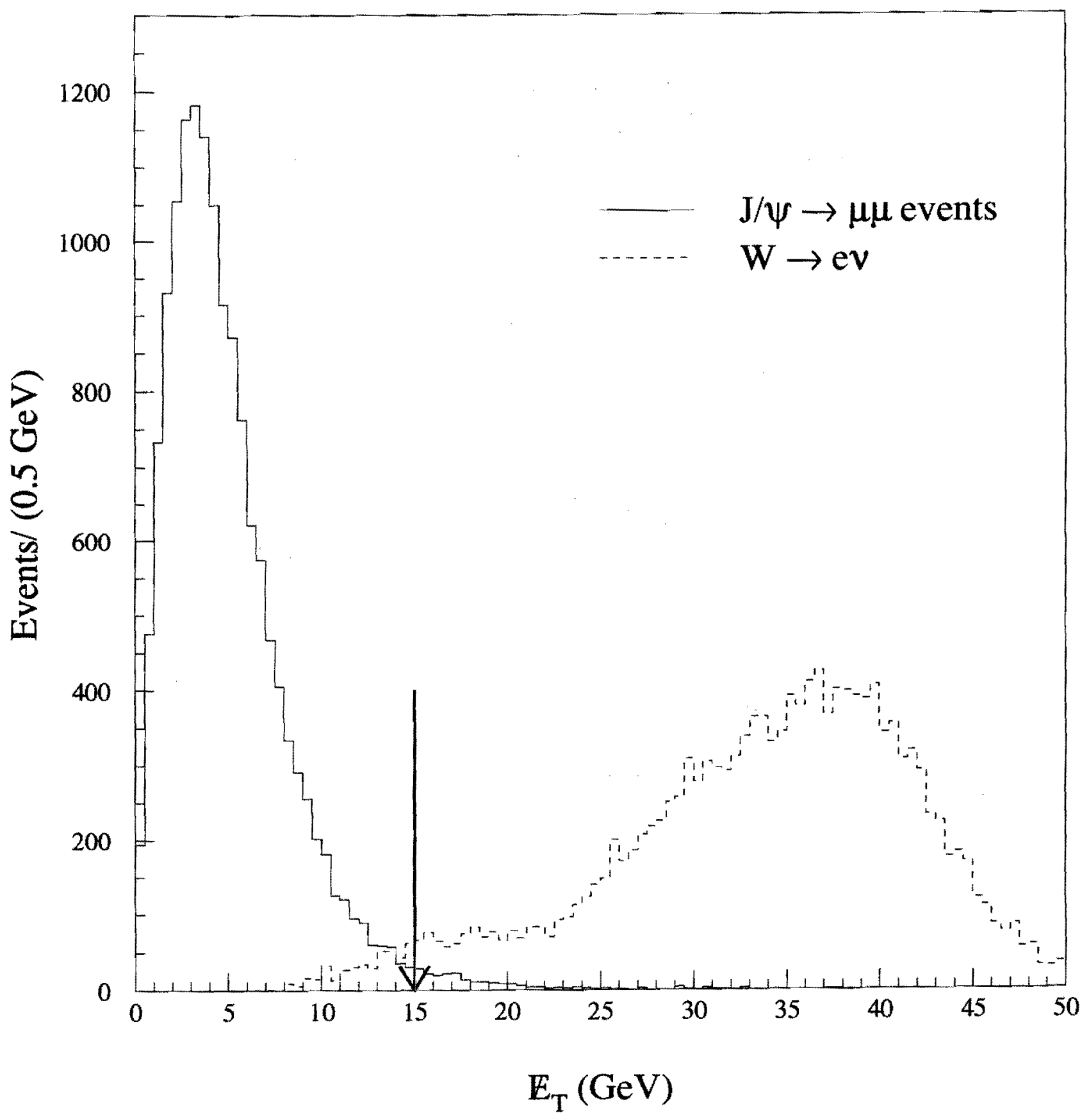

Figure 4.20: The missing transverse energy distributions for $J / \psi \rightarrow \mu \mu$ (solid) and $W \rightarrow e \nu$ events (dotted). 


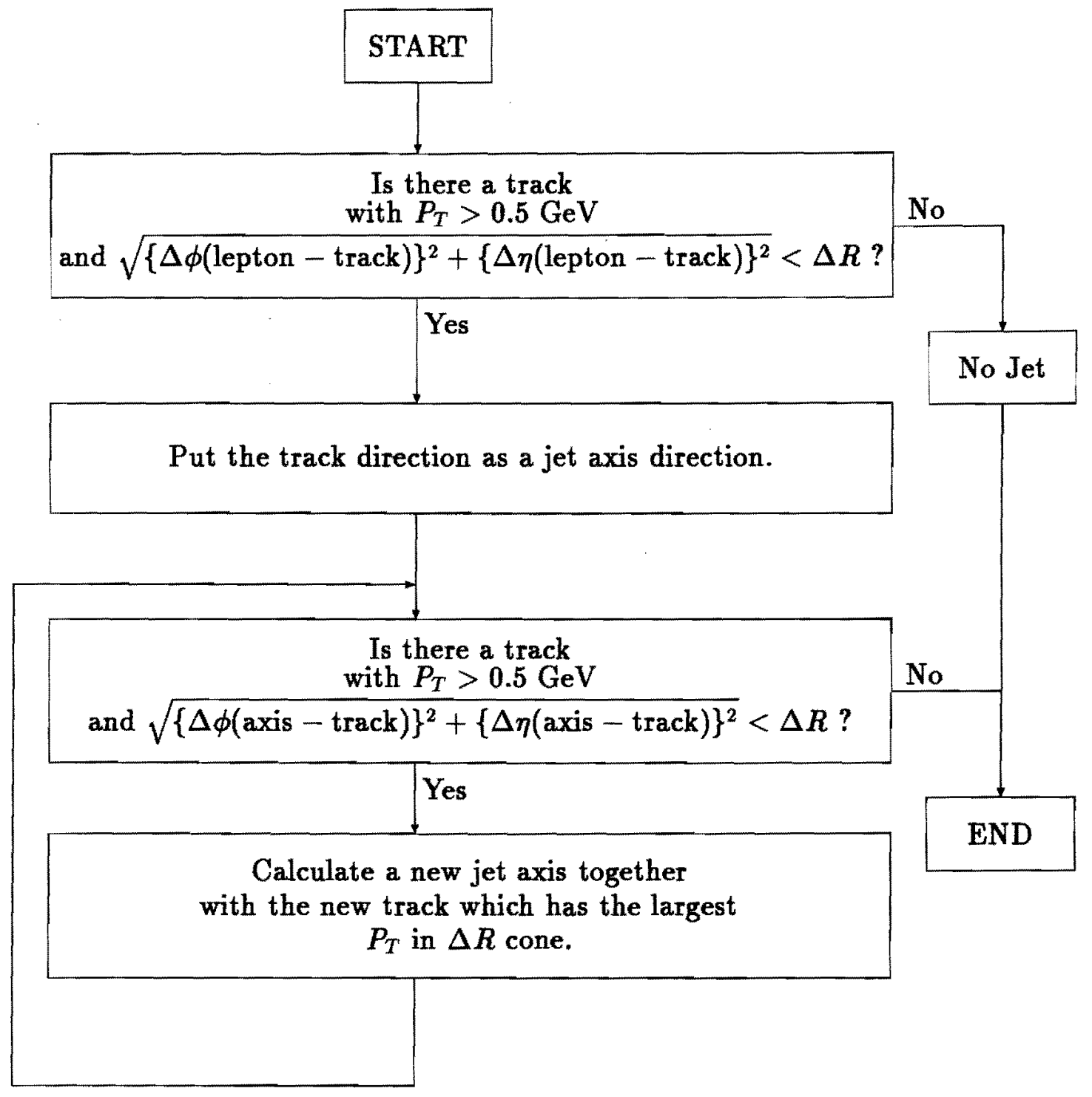

Figure 4.21: Jet axis finding algorithm. 

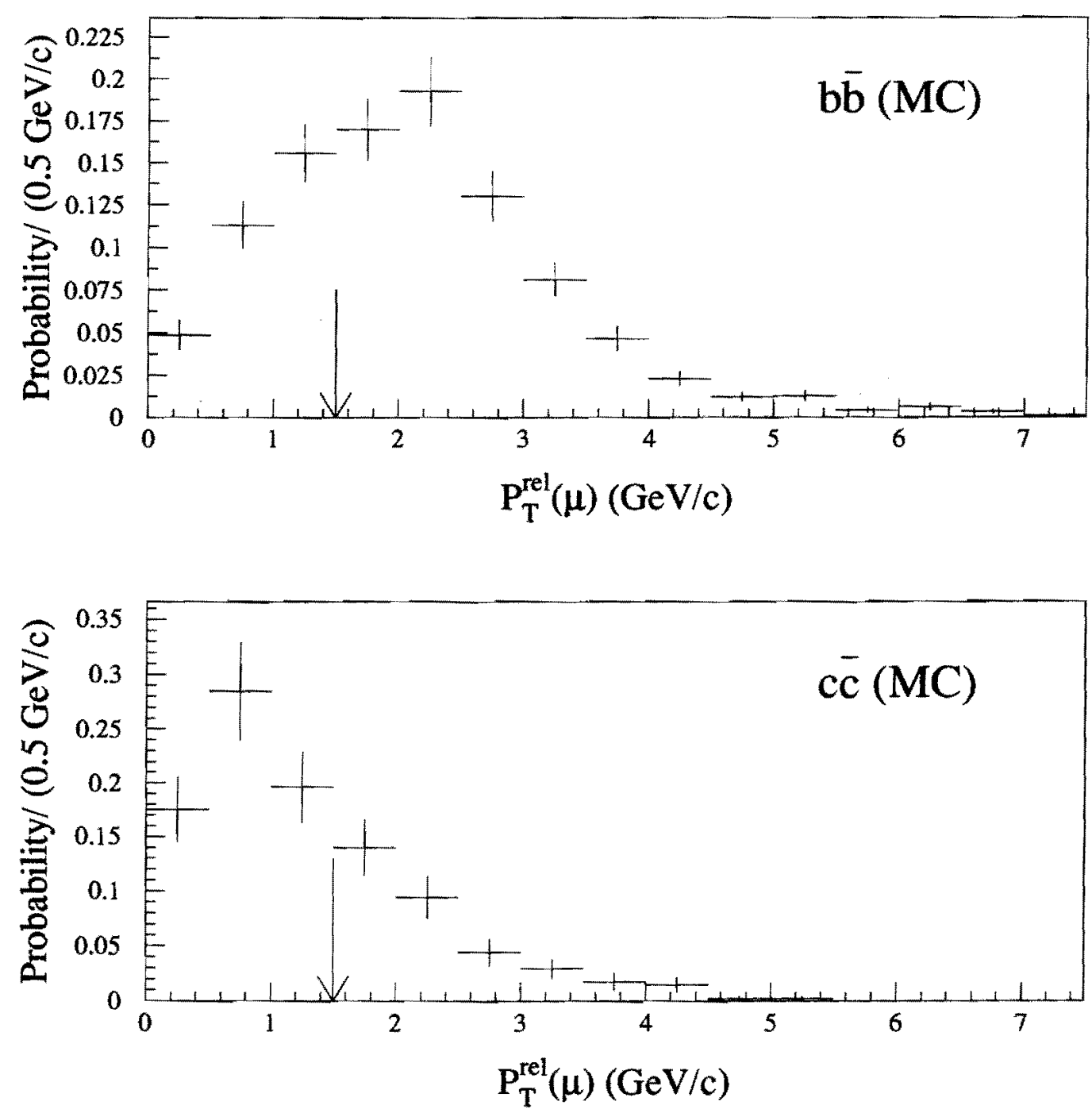

Figure 4.22: The muon $P_{T}^{\text {rel }}$ distributions for $b \bar{b}$ and $c \bar{c}$ Monte Carlo events. 

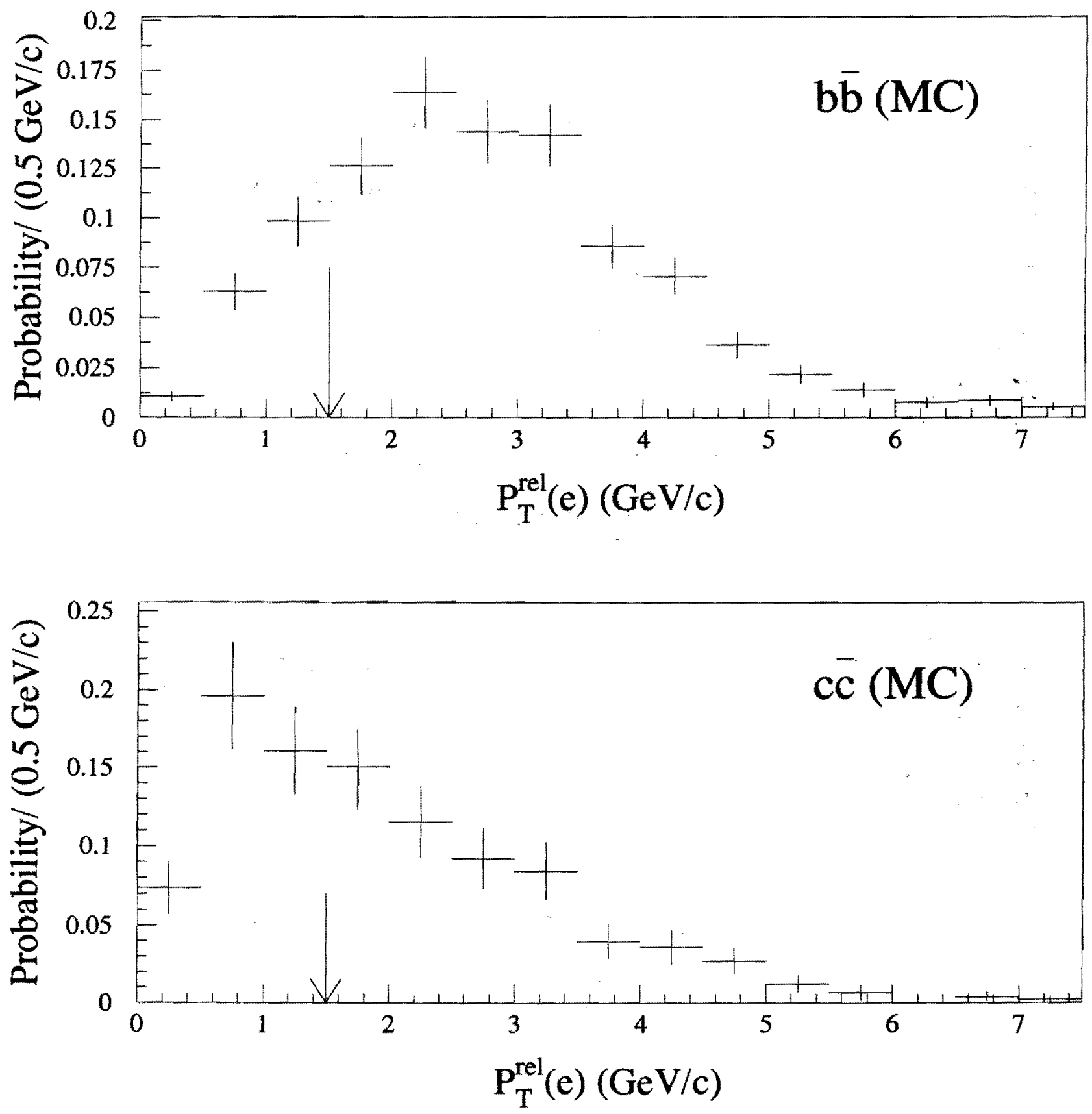

Figure 4.23: The electron $P_{T}^{\text {rel }}$ distributions for $b \bar{b}$ and $c \bar{c}$ Monte Carlo events. 

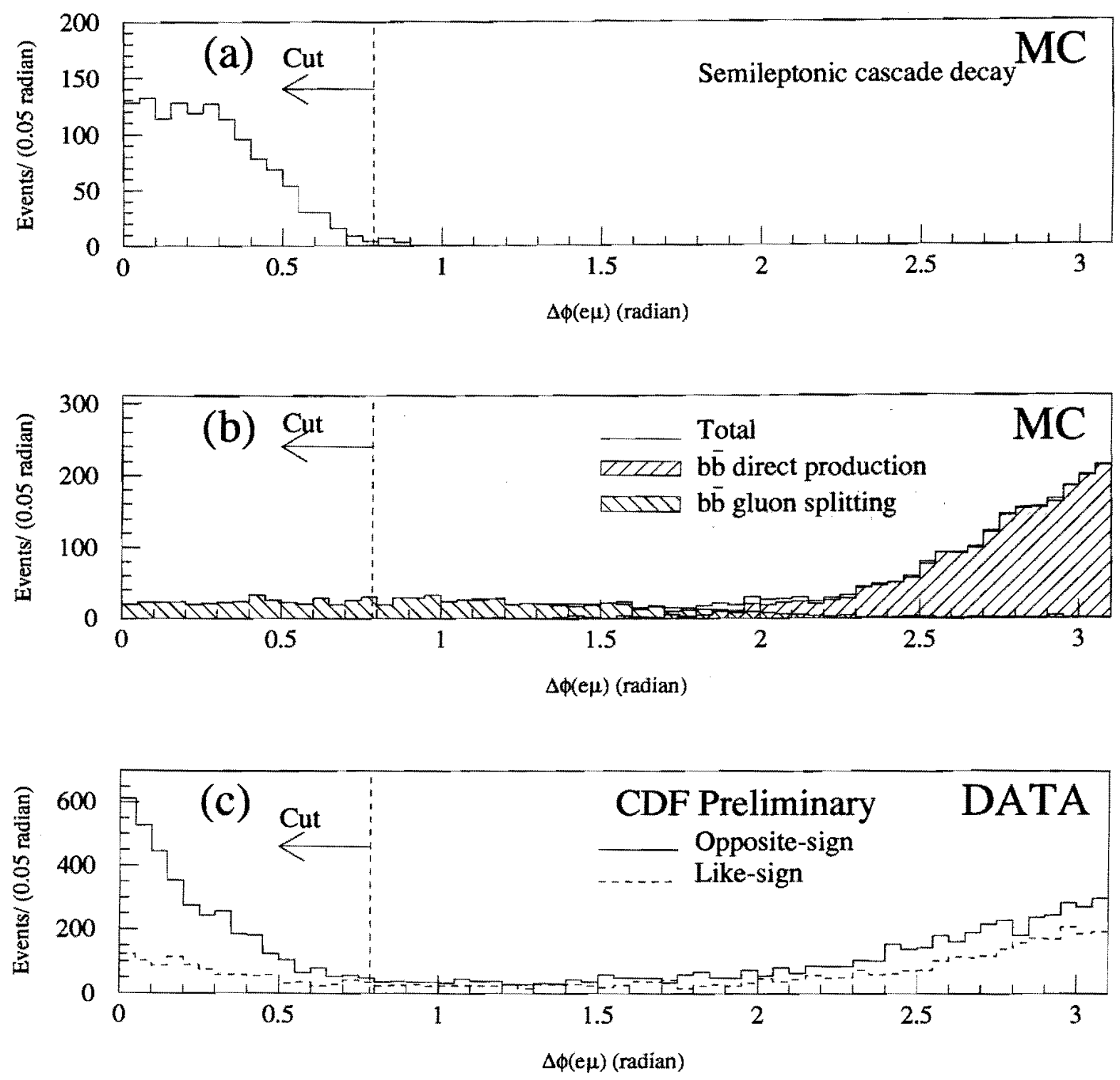

Figure 4.24: (a) Monte Carlo distribution of $\Delta \phi(e \mu)$ for semileptonic cascade decays. (b) Monte Carlo distribution of $\Delta \phi(e \mu)$ for $b \bar{b}$ direct production, $b \bar{b}$ production from gluon splitting and all (solid line). (c) The $\Delta \phi(e \mu)$ distribution for opposite-sign (solid line) and like-sign (dashed line) $e \mu$ events. 


\section{Chapter 5}

\section{Backgrounds and Systematic Uncertainties}

\subsection{Fraction of fake $e \mu$ events}

The observed $e \mu$ events can be classified into four categories as listed in Table 5.1. In terms of them, the fake muon and the fake electron fractions of the observed $e \mu$ events are written as

$$
F_{\mu}=\frac{(b)+(d)}{(a)+(b)+(c)+(d)}
$$

\begin{tabular}{ll}
\hline \hline Muon-Electron & \\
\hline Real - Real & (a) \\
Fake- Real & (b) \\
Real - Fake & (c) \\
Fake-Fake & (d) \\
\hline \hline
\end{tabular}

Table 5.1: Four categories of the observed $e \mu$ events. 
and

$$
F_{e}=\frac{(c)+(d)}{(a)+(b)+(c)+(d)}
$$

respectively. The fake muon fraction of the fake electron sample is given by

$$
F_{\mu}^{\prime}=\frac{(d)}{(c)+(d)}
$$

Using the above equations, the fake $e \mu$ fraction of the observed $e \mu$ events is expressed as

$$
\begin{aligned}
F_{e \mu} & \equiv \frac{(b)+(c)+(d)}{(a)+(b)+(c)+(d)} \\
& =F_{\mu}+F_{e}-F_{e} \cdot F_{\mu}^{\prime}
\end{aligned}
$$

The fake muon and the fake electron fractions, $F_{\mu}$ and $F_{e}$, are estimated by the same method. We explain the method for muons as an example. Let us put $N$ to be the number of observed $e \mu$ 's which is 1710 for OS and 861 for LS. In order to estimate $F_{\mu}$, we loosen the muon identification cut, and the new identification cut is called a loose muon identification cut. Then the event selection is performed again, resulting in a larger number of $e \mu$ events, $N^{\prime}$. The $N^{\prime}$ can be written as

$$
N^{\prime}=N_{f}+N_{r}
$$

where $N_{f}$ and $N_{r}$ are the numbers of fake and real muons which passed the loose identification cut. Putting $P_{f}$ and $P_{r}$ to be the probabilities that the fake and the real muons pass the original muon identification cut, $N$ is expressed as

$$
N=N_{f} \cdot P_{f}+N_{r} \cdot P_{r}
$$

Since $N^{\prime}$ and $N$ are observed numbers, the $N_{f}$ and $N_{r}$ are estimated if $P_{f}$ and $P_{r}$ are 
known. Then the fake muon fraction is obtained as

$$
F_{\mu}=\frac{N_{f} \cdot P_{f}}{N}
$$

Estimation of $F_{\mu}^{\prime}$ is done similarly using low quality electrons as the fake electron sample.

\subsubsection{Fake muon fraction}

As the loose muon identification selection, we removed the requirement that muon candidates have a CMP stub and the other cut parameters were the same. Applying the $e \mu$ event selection to the data with the loose muon identification, we obtained $N^{\prime}(O S)=2122$ and $N^{\prime}(L S)=1175$. Estimation of $P_{f}$ and $P_{r}$ is done by using data samples different from the $e \mu$ sample.

\section{Probability for fake muons $P_{f}$}

Fake muons are considered to originate from two sources: (1) Non-interacting punchthrough (NIP) and (2) decay in flight (DIF) of hadrons. First we roughly estimate $P_{f}$ before detailed analysis. We know from the previous CDF experiment [47] that the numbers of NIP and DIF particles are almost the same at the CMU chambers. The number of NIP particles at the CMP is almost zero due to hadron absorption in the steel walls between the CMU and the CMP chambers. And the efficiency of the CMP chambers for DIF

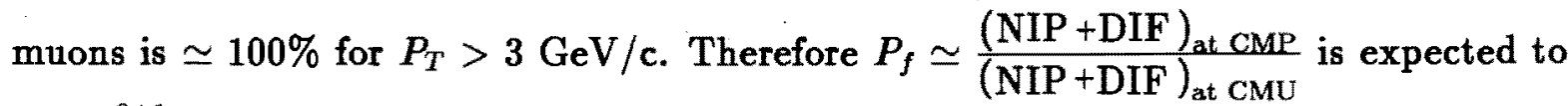
be $\simeq \frac{0+1}{1+1}=0.5$.

To be more quantitative, the probability, $P_{f}$, was estimated in two different ways. Firstly, it was estimated from minimum-bias data corresponding to $18 \mathrm{pb}^{-1}$ in RUN Ia. We assumed there was no real muon in the minimum bias data. We found 63 and 125 tracks which passed the muon identification cut and the loose one, respectively. Then we have $P_{f}=63 / 125=0.504 \pm 0.045$.

Secondly, $P_{f}$ was estimated by using $K_{s}^{0} \rightarrow \pi \pi$ and $\phi \rightarrow K K$ decays in the low $P_{T}$ 
muon inclusive sample in JPSI STREAM 1 . We reconstruct the $K_{s}^{0} \rightarrow \pi \pi$ decay using a muon candidate track and one of oppositely charged 3D tracks, assuming that both tracks are pions. The oppositely charged 3D track which gives the $\pi \pi$ invariant mass closest to the $K_{s}^{0}$ mass was chosen. The secondary vertex of the two tracks was required to be more than $5 \mathrm{~cm}$ away from the primary vertex on $r-\phi$ plane. Figure 5.1 shows the invariant mass distribution. The dashed (solid) line histogram corresponds to the case where the muon candidate track passed the muon identification cut (the loose one). The $P_{f}$ for pions is given by a ratio of the number of $K_{s}^{0}$ decays in the dashed histogram to that in the solid one. We fitted a Gaussian + cubic polynomial function to the invariant mass distribution in the region between 0.3 and $0.7 \mathrm{GeV} / \mathrm{c}^{2}$. Defining a region between 0.45 and $0.55 \mathrm{GeV} / \mathrm{c}^{2}$ as a $K_{s}^{0}$ signal region and subtracting background using the cubic polynomial function, we obtained $P_{f}=0.498 \pm 0.011$ for pions.

We did a similar analysis for the $\phi \rightarrow K K$ decay. In this case, two tracks were assumed to be kaons and the secondary vertex cut was not required. The invariant mass distribution is shown in Fig. 5.2. We obtained $P_{f}=0.498 \pm 0.016$ for kaons.

Assuming the $K / \pi$ ratio in the sample to be $0.240 \pm 0.120[48,49]$, we estimated $P_{f}=0.498 \pm 0.010$ for hadrons.

The two independent methods described above using the minimum bias data and the invariant mass peaks of $K_{s}^{0}$ and $\phi$ give consistent values. We took the weighted average of them and obtained

$$
P_{f}=0.498 \pm 0.009 \quad \text { for fake muons. }
$$

\section{Probability for real muons $P_{r}$}

We used $J / \psi \rightarrow \mu \mu$ decays in the low $P_{T}$ dimuon sample in order to estimate $P_{r}$. The invariant mass distribution for dimuons is shown in Figs. 5.3 (a) and (b). In Fig. 5.3 (a), one of the two muons passed the muon identification cut and the other muon passed the loose one. In Fig. 5.3 (b), both of the two muons passed the muon identification cut. 
A Gaussian + constant function was fitted to these histograms in a region of invariant mass between 2.8 and $3.4 \mathrm{GeV} / \mathrm{c}^{2}$. The probability for real muons, $P_{r}$, is given by

$$
P_{r}=\frac{2 N_{b}}{N_{a}+N_{b}}
$$

where $N_{a}$ and $N_{b}$ are the numbers of $J / \psi$ decays in Figs. 5.3 (a) and (b) after subtracting backgrounds estimated from the constant term (See Appendix A for derivation of Eq. (5.9)). Looking at a region between 3.0 and $3.2 \mathrm{GeV} / \mathrm{c}^{2}$ around the $J / \psi$ mass, we obtained

$$
P_{r}=0.967 \pm 0.002 \quad \text { for real muons. }
$$

\section{Fake muon fraction $F_{\mu}$}

Since we obtained $N, N^{\prime}, P_{r}$ and $P_{f}$, we could solve Eqs. (5.5) and (5.6) for $N_{f}$, which was found 313 for OS and 271 for LS. Using Eq. (5.7), we obtained

$$
F_{\mu}(O S)=0.212 \pm 0.014(\text { stat. }) \pm 0.008(\text { syst. })
$$

and

$$
F_{\mu}(L S)=0.339 \pm 0.025 \text { (stat.) } \pm 0.013 \text { (syst.) }
$$

where the statistical uncertainties come from $N$ and $N^{\prime}$, and the systematic uncertainties come from $P_{r}$ and $P_{f}$.

\subsubsection{Fake electron fraction}

We loosened the $H A D / E M$ cut value from 0.04 to 0.1 for loose electron identification cut. The other cut parameters were left the same as those in Table 4.7. We obtained $N^{\prime}(O S)=1845$ and $N^{\prime}(L S)=969$. 


\section{Probability for fake electrons $P_{f}$}

There are several sources of background which mimics electron signature in the detector. One of them is a charged pion interacting in the EM calorimeter. Most of charged pions are discriminated by the fact that the energy deposition in the hadronic calorimeter is large while that in the EM calorimeter is small. But some of charged pions interact and deposit a large part of their energy in the EM calorimeter. Since a charged pion leaves an associated track in the CTC, it looks like an electron. Another source of fake electrons is a photon with an overlapping charged track. The photon makes an energy deposition in the EM calorimeter, its track looks associated to that EM energy deposition. This also mimics the electron signature. On top of these fake electrons, there are electrons which are not related to hard collisions, such as electrons from photon conversions $(\gamma \rightarrow e e)$.

In this selection, we consider two sources of fake electrons: (1) photons overlapped by charged hadrons in the EM calorimeter and (2) charged hadrons which deposited a significant amount of energy in the EM calorimeter. Photon conversions will be dealt with in the next section.

We studied photon + charged hadron overlap by removing the cut on position difference at the CES, $\triangle X(C E S)$, from the electron identification, and estimated $P_{f}$ for electron candidates with large $\triangle X(C E S)$, most of which were considered to be the photon+ charged hadron overlap. Figure. 5.4 (a) shows the $H A D / E M$ distribution for electron candidates with $\Delta X(C E S)>6 \mathrm{~cm}$. The probability, $P_{f}$, for a photon with an overlapping charged track to pass the electron identification cut was estimated from a ratio of the number of events in $H A D / E M<0.04$ (hatched area) to that in $H A D / E M<0.1$. We plot $P_{f}^{-}$as a function of the lower cut value of $\triangle X(C E S)$ in Fig. 5.4 (b). We take the value of $0.606 \pm 0.025$ for $\Delta X(C E S)>6 \mathrm{~cm}$ as $P_{f}$ for photon + charged track overlap.

We used a Monte Carlo to study charged hadrons which deposited a significant amount of energy in the EM calorimeter. We generated charged pions with $P_{T}=$ 5 and $7 \mathrm{GeV} / \mathrm{c}$ and let them pass through the CDFSIM detector simulation package. 
Figures 5.5 (a) and (b) show the $H A D / E M$ distributions for pions passing the loose electron identification selection for $P_{T}$ of 5 and $7 \mathrm{GeV} / \mathrm{c}$, respectively. We obtained $P_{f}=0.482 \pm 0.036(0.394 \pm 0.018)$ for pions with $P_{T}=5(7) \mathrm{GeV} / \mathrm{c}$.

Since we do not know the mixture ratio between the two sources, we assume the true value of $P_{f}$ lies between the upper and lower extremes in the estimates, i.e., $0.606+0.025$ and $0.394-0.018$. That is, we take

$$
P_{f}=0.50 \pm 0.13 \quad \text { for fake electrons. }
$$

\section{Probability for real electrons $P_{r}$}

We estimated $P_{r}$ for real electrons by a very similar way for muons. We reconstructed $J / \psi \rightarrow e e$ decays from two tracks of electrons in the dilepton sample. The invariant mass distribution is different from that for muons due to the bremsstrahlung of electrons. Since we are interested in the number of $J / \psi$ 's and not in the shape of the distribution itself, we did not correct it for the bremsstrahlung. We fitted a constant + an asymmetric Gaussian which has two different $\sigma$ 's on the right and left side of the peak to the distribution in the region between 2.3 and $3.8 \mathrm{GeV} / \mathrm{c}^{2}$. We defined the signal region as the invariant mass region between 2.9 and $3.2 \mathrm{GeV} / \mathrm{c}^{2}$, and from the numbers of backgrounds subtracted $J / \psi \rightarrow$ ee events, we obtained

$$
P_{r}=0.983 \pm 0.004 \quad \text { for real electrons. }
$$

\section{Fake electron fraction}

Putting the numbers for $P_{f}, P_{r}, N$ and $N^{\prime}$ in Eqs. (5.5) and (5.6), $N_{f}$ was found to be 72 for OS and 78 for LS. Then, from Eq 5.7 we obtained $F_{e}(O S)=0.062 \pm 0.007(s t a t$. $) \pm$ 0.033 (syst.) and $F_{e}(L S)=0.110 \pm 0.013$ (stat.) \pm 0.059 (syst.). 


\subsubsection{Contribution of photon conversions}

We now consider photon conversion electrons as another fake electron source. Although most of photon conversion electrons were removed by the electron identification selection, some of them remain in the $e \mu$ sample when one of the two electron tracks is missed. Because one of the two conversion electrons have lower $P_{T}$ than threshold $P_{T}$ of track which is reconstructed by CTC data.

This contribution was estimated by using transverse momentum distributions for the photon conversion electron candidates in the $e \mu$ sample and a Monte Carlo [17, 50]. Photons in the Monte Carlo are generated with the transverse momentum spectra which is estimated by real photon conversion sample. The photons are converted to two electrons by a detector simulator.

Figure 5.7 shows the momentum distribution of an associated track with tagged electrons for the photon conversion in real data. The transverse momentum distribution of associated tracks for the Monte Carlo data in a region of $1<P_{T}($ track $)<5 \mathrm{GeV} / \mathrm{c}$ is normalized to that for photon conversion events. The number of photon conversion events where one of two electrons is missed is estimated by subtracting the number of events for photon conversion from the normalized Monte Carlo events.

The contribution of $\gamma$ conversion electrons to fake electrons is estimated as a fraction of the subtracted number to number of electron candidates and it turned out to be $(2.00 \pm 0.64) \%$. Adding this value to previous $F_{e}$ 's,

$$
F_{e}(O S)=0.082 \pm 0.010(\text { stat. }) \pm 0.034(\text { syst. })
$$

and

$$
F_{e}(L S)=0.111 \pm 0.015(\text { stat. }) \pm 0.059(\text { syst } .)
$$

are obtained. 


\subsubsection{Fake $e \mu$ fraction $F_{e \mu}$}

In order to estimate $F_{e \mu}$, we need to know the fake muon fraction in the fake electron sample, $F_{\mu}^{\prime}$, as can be seen in Eq. (5.4). We considered electron candidates with $H A D / E M$ between 0.04 and 0.1 as a fake electron sample and estimated $F_{\mu}^{\prime}$. We counted $N$ and $N^{\prime}$ in the fake electron sample and obtained $N=135$ and $N^{\prime}=202$ for OS events; $N=108$ and $N^{\prime}=171$ for LS events. Using $N, N^{\prime}$ and, $P_{r}=0.967 \pm 0.002$ and $P_{f}=0.498 \pm 0.009$ given in Eqs. 5.8 and 5.10, we obtained

$$
F_{\mu}^{\prime}(O S)=0.474 \pm 0.078
$$

and

$$
F_{\mu}^{\prime}(L S)=0.564 \pm 0.098
$$

Substituting $F_{\mu}, F_{e}$ and $F_{\mu}^{\prime}$ into Eq. (5.4), we obtained

$$
F_{e \mu}(O S)=0.255 \pm 0.016(\text { stat. }) \pm 0.021(\text { syst. })
$$

and

$$
F_{e \mu}(L S)=0.396 \pm 0.028(\text { stat. }) \pm 0.031(\text { syst. }) .
$$

We summarize the fake lepton fractions in Table 5.2.

\subsubsection{Asymmetry of fake $e \mu$ events}

The number of fake $e \mu$ events is 436 for OS and 340 for LS, and there seems to be an asymmetry, $436 / 340=1.28 \pm 0.19$. In the fake $e \mu$ events, events of fake muon + real electron are dominant. Since the real electron $P_{T}$ 's are high enough to ensure that most of the real electrons are decay products of $b$ hadrons, the main source of the fake $e \mu$ events is considered to be $b \bar{b}$ events. We study the origin of the asymmetry using $b \bar{b}$ Monte Carlo events. 


\begin{tabular}{|c|c|c|c|}
\cline { 2 - 4 } & \multicolumn{3}{c|}{ Opposite-sign } \\
\cline { 2 - 4 } & Muon & Electron & $\begin{array}{c}\text { Muon } \\
\text { (Fake electron sample) }\end{array}$ \\
\hline \hline$N$ & \multicolumn{2}{|c|}{1710} & 135 \\
\hline$N^{\prime}$ & 2122 & 1845 & 202 \\
\hline$N_{f} \times P_{f}$ & $362 \pm 17$ & $140 \pm 58$ & - \\
\hline$N_{r} \times P_{r}$ & $1348 \pm 45$ & $1570 \pm 71$ & - \\
\hline$F_{\mu}, F_{c}$ & $0.212 \pm 0.016$ & $0.082 \pm 0.035$ & - \\
\hline$F_{\mu}^{\prime}$ & - & - & $0.474 \pm 0.078$ \\
\hline$F_{e \mu}$ & \multicolumn{3}{|c|}{$0.255 \pm 0.026$} \\
\hline$N \times F_{e \mu}$ & \multicolumn{3}{|c|}{$436 \pm 44$} \\
\hline
\end{tabular}

\begin{tabular}{|c|c|c|c|}
\cline { 2 - 4 } & \multicolumn{3}{c|}{ Like-sign } \\
\cline { 2 - 4 } & Muon & Electron & $\begin{array}{c}\text { Muon } \\
\text { (Fake electron sample) }\end{array}$ \\
\hline \hline$N$ & \multicolumn{2}{|c|}{861} & 108 \\
\hline$N^{\prime}$ & 1175 & 969 & 171 \\
\hline$N_{f} \times P_{f}$ & $292 \pm 15$ & $111 \pm 51$ & - \\
\hline$N_{r} \times P_{r}$ & $569 \pm 33$ & $750 \pm 59$ & - \\
\hline$F_{\mu}, F_{e}$ & $0.339 \pm 0.028$ & $0.130 \pm 0.060$ & - \\
\hline$F_{\mu}^{\prime}$ & - & - & $0.564 \pm 0.098$ \\
\hline$F_{e \mu}$ & \multicolumn{3}{|c|}{$\mathbf{0 . 3 9 6 \pm 0 . 0 4 2}$} \\
\hline$N \times F_{e \mu}$ & \multicolumn{3}{|c|}{$340 \pm 37$} \\
\hline
\end{tabular}

Table 5.2: Summary of the fake lepton fraction estimation. The uncertainties include both the statistical and systematic uncertainties. 
We generated $b \bar{b}$ events using ISAJET, and the $b$ and $\bar{b}$ hadrons were decayed by CLEOMC. First, to simulate real lepton + fake lepton events, all $b$ hadrons were forced to decay to leptons and all $\bar{b}$ hadrons were forced to decay to hadrons only. The $P_{T} \mathrm{~s}$ of leptons and charged hadrons were required to be greater than $3 \mathrm{GeV} / \mathrm{c}$ since the $P_{T}$ threshold for leptons is $3 \mathrm{GeV} / \mathrm{c}$ in this analysis. The pseudo-rapidities of leptons and hadrons were required to be $|\eta|<0.6$, corresponding to the central muon detector region. Since our electron-muon events are required that the azimuthal opening angle between the leptons is greater than $45^{\circ}$, the azimuthal opening angle of a lepton and a hadron is required to be greater than $45^{\circ}$. After these kinematical cuts were applied, the number of OS lepton-hadron pairs was 749 and that of LS lepton-hadron pairs was 518. Then the asymmetry in the OS and LS real lepton + fake lepton pairs is calculated to be $749 / 518=1.446 \pm 0.083$. Second, to simulate fake lepton + fake lepton events, we generated $b \bar{b}$ events and forced both the $b$ and $\bar{b}$ quarks to decay to hadrons. After applying the same kinematical cuts for hadrons, the number of OS hadron-hadron pairs was 602 and that of LS hadron-hadron pairs was 561 . The asymmetry in this case was $602 / 561=1.073 \pm 0.063$

There is an asymmetry in the fake lepton + real lepton Monte Carlo $b \bar{b}$ events, but we can not say that there is an asymmetry in the fake lepton + fake lepton Monte Carlo $b \bar{b}$ events. Since the observed fake $e \mu$ events include fake lepton + real lepton events and fake lepton + fake lepton events in a certain ratio, we can expect the asymmetry will be in between 1 and 1.446. This result is consistent with the observed asymmetry of 1.28 .

In order to see where the Monte Carlo asymmetry comes from, we studied multiplicities of charged particles from $B$ and $\vec{B}$ mesons. For that end, $b \vec{b}$ events were generated and, $b$ and $\bar{b}$ hadrons were decayed by the default branching ratio of CLEOMC. Then the multiplicities of charged particles were counted for each $B$ meson. Figures 5.8 - 5.13 show the multiplicity distributions for $B_{u}^{-}, \bar{B}_{d}^{0}, \bar{B}_{s}^{0}, B_{u}^{+}, B_{d}^{0}$ and $B_{s}^{0}$ mesons, respectively. Histogram (a)'s in these figures show the multiplicity of charged particles before the 


\begin{tabular}{|c|c|c|}
\hline \hline Quark & Meson & Charge asymmetry (+/-) \\
\hline & $B_{u}^{-}$ & $0.35 \pm \mathbf{0 . 0 1}$ \\
$b$ & $\bar{B}_{d}^{0}$ & $0.74 \pm \mathbf{0 . 0 2}$ \\
& $\widetilde{B}_{s}^{0}$ & $1.17 \pm \mathbf{0 . 0 6}$ \\
\hline \multirow{3}{*}{$\bar{b}$} & $B_{u}^{+}$ & $2.92 \pm \mathbf{0 . 1 0}$ \\
& $B_{d}^{0}$ & $1.27 \pm 0.04$ \\
& $B_{s}^{0}$ & $\mathbf{0 . 8 3} \pm \mathbf{0 . 0 5}$ \\
\hline \hline
\end{tabular}

Table 5.3: The daughter hadron charge asymmetry for each $B$ meson. The uncertainties are due to the statistics of Monte Carlo events.

kinematical cut is applied. The number of charged particles produced from charged and neutral $B$ mesons are odd and even, as they should be. The numbers of tracks (hadrons or leptons) after requiring the cuts on $P_{T}$ and $|\eta|$ are almost 1 for any $B$ mesons, as shown in histogram (b)'s. Histogram (c)'s are the same plots for hadrons only, and the distributions are essentially the same as for tracks. Since the charged hadron multiplicity after the cut is one most of the time, we calculate the daughter hadron charge asymmetry for each $B$ meson using multiplicity-one event only. We summarize the asymmetries in Table 5.3. Assuming the production ratio of $B_{u}: B_{d}: B_{s}$ to be $1: 1: 0.3$ $[51,52,53]$, the ratios of positive hadrons to negative hadrons are 0.63 and 1.40 for $b$ and $\bar{b}$ quarks, respectively. When a $b$ quark in $b \bar{b}$ events decays semileptonically to a negatively charged lepton and the other $\vec{b}$ quark decays into hadrons, we are more likely to find a positively charged hadron from the $\bar{b}$ quark. This creates the asymmetry in real lepton + fake lepton events. These studies show the asymmetry in fake $e \mu$ events originates from physical processes and our event selection.

\subsection{Fraction of sequential decays}

The fraction of sequential decays, $f_{s}$, was estimated from Monte Carlo events. 


\subsubsection{Monte Carlo of $b \bar{b}$ events}

The $b \bar{b}$ events were generated by ISAJET (Version 7.06) with $P_{T}>2.9 \mathrm{GeV} / \mathrm{c}$ for both $b$ and $\bar{b}$. The gluon splitting events were generated by ISALEP (Version 7.06) with $P_{T}>9.8 \mathrm{GeV} / \mathrm{c}$ for gluons that produced $b \bar{b}$ pairs. Quarks and gluons were hadronized and decayed in the ISAJET and ISALEP generators, but the $B$ and $\bar{B}$ meson decays were replaced by those simulated using CLEOMC (Version 9.00). Finally the $b \bar{b}$ events were passed through the QFL (offline version 7.10) detector simulator.

\subsubsection{Estimation of the $f_{s}$}

To simulate Process 1 (Table 4.1), all $b$ and $\bar{b}$ hadrons were forced to decay to leptons. For Process 2, all $b$ hadrons were forced to decay to leptons and $\bar{b}$ hadrons were decayed following the CLEOMC decay table. In the decay products from $\bar{b}$ hadrons, $\bar{c}$ hadrons were forced to decay to leptons. Particles and anti-particles in Process 2 were exchanged for Process 3.

The numbers of Monte Carlo events that passed the $e \mu$ event selection are shown in Tables 5.4 to 5.12 for different structure functions and Peterson fragmentation parameters. The luminosities for generated events are also shown in the tables. The fraction of sequential decays is given by a ratio of the number of events for Processes 2 and 3 to that for Process 1 , where the number of events is normalized by the luminosity, taking into account the branching ratio, $\operatorname{Br}(b \rightarrow l+X)=0.110 \pm 0.005[4]$ and $\operatorname{Br}(c \rightarrow l+X)$. The branching ratio $B r(c \rightarrow l+X)$ for $D^{+}$and $D^{0}$ are used $0.172 \pm 0.019$ and $0.0885 \pm 0.0143[4]$, respectively. For $D_{s}^{+}$and $c$ baryon, the branching ratio are estimated from $\mathrm{Br}\left(D^{+} \rightarrow e+\right.$ anything $)=0.172 \pm 0.019$ and, the mean life, $\tau\left(D^{+}\right)=1.057 \pm 0.015, \tau\left(D_{s}^{+}\right)=0.467 \pm 0.017$ and $\tau\left(\Lambda_{c}^{+}\right)=0.200 \pm 0.010$ [4]. The estimated branching ratio for $D_{s}^{+}$and $c$ baryon are $0.076 \pm 0.019$ and $0.033 \pm 0.010$, respectively.

The structure functions used in the above calculation are Martin-Roberts-Stirling (MRS) D0-' structure function [54, 55], Eichten-Hinchliffe-Lane-Quigg (EHLQ) struc- 


\begin{tabular}{|c|c|c|c|c|c|}
\hline & & \multicolumn{2}{|c|}{ ISAJET } & \multicolumn{2}{|c|}{ ISALEP } \\
\hline & & $\begin{array}{c}\text { Number } \\
\text { of } \\
\text { events }\end{array}$ & $\begin{array}{l}\text { Luminosity } \\
\qquad\left(\mathrm{pb}^{-1}\right)\end{array}$ & $\begin{array}{c}\text { Number } \\
\text { of } \\
\text { events }\end{array}$ & $\begin{array}{l}\text { Luminosity } \\
\qquad\left(\mathrm{pb}^{-1}\right)\end{array}$ \\
\hline \multicolumn{2}{|l|}{ Process 1} & 1024 & 0.742 & 59 & 0.309 \\
\hline \multirow{4}{*}{ Process 2} & $\bar{b} \rightarrow \overline{D^{0}} \rightarrow l$ & 33 & \multirow{4}{*}{0.729} & 5 & \multirow{4}{*}{0.336} \\
\hline & $\bar{b} \rightarrow D^{-} \rightarrow l$ & 16 & & 0 & \\
\hline & $\bar{b} \rightarrow D_{s}^{-} \rightarrow l$ & 5 & & 1 & \\
\hline & $\bar{b} \rightarrow$ baryon $_{\bar{c}} \rightarrow l$ & 5 & & 0 & \\
\hline \multirow{4}{*}{ Process 3} & $b \rightarrow D^{0} \rightarrow l$ & 24 & \multirow{4}{*}{0.663} & 2 & \multirow{4}{*}{0.296} \\
\hline & $b \rightarrow D^{+} \rightarrow l$ & 10 & & 1 & \\
\hline & $b \rightarrow D_{s}^{+} \rightarrow l$ & 6 & & 1 & \\
\hline & $b \rightarrow$ baryon $_{c} \rightarrow l$ & 5 & & 0 & \\
\hline
\end{tabular}

Table 5.4: The numbers of $\varepsilon \mu$ events which passed the $e \mu$ event selection and the corresponding luminosities. The structure function is MRSD0 -' and the Peterson parameter is set to 0.003 . 


\begin{tabular}{|c|c|c|c|c|c|}
\hline & & \multicolumn{2}{|c|}{ ISAJET } & \multicolumn{2}{|c|}{ ISALEP } \\
\hline & & $\begin{array}{c}\text { Number } \\
\text { of } \\
\text { events }\end{array}$ & $\begin{array}{l}\text { Luminosity } \\
\qquad\left(\mathrm{pb}^{-1}\right)\end{array}$ & $\begin{array}{c}\text { Number } \\
\text { of } \\
\text { events }\end{array}$ & $\begin{array}{l}\text { Luminosity } \\
\qquad\left(\mathrm{pb}^{-1}\right)\end{array}$ \\
\hline \multicolumn{2}{|l|}{ Process 1} & 1053 & 0.790 & 46 & 0.308 \\
\hline \multirow{4}{*}{ Process 2} & $\bar{b} \rightarrow \bar{D}^{0} \rightarrow l$ & 21 & \multirow{4}{*}{0.624} & 2 & \multirow{4}{*}{0.305} \\
\hline & $\bar{b} \rightarrow D^{-} \rightarrow l$ & 11 & & 2 & \\
\hline & $\bar{b} \rightarrow D_{s}^{-} \rightarrow l$ & 2 & & 0 & \\
\hline & $\bar{b} \rightarrow$ baryon $_{\bar{c}} \rightarrow l$ & 5 & & 0 & \\
\hline \multirow{4}{*}{ Process 3} & $b \rightarrow D^{0} \rightarrow l$ & 30 & \multirow{4}{*}{0.660} & 2 & \multirow{4}{*}{0.319} \\
\hline & $b \rightarrow D^{+} \rightarrow l$ & 13 & & 2 & \\
\hline & $b \rightarrow D_{s}^{+} \rightarrow l$ & 6 & & 0 & \\
\hline & $b \rightarrow$ baryon $_{c} \rightarrow l$ & 2 & & 0 & \\
\hline
\end{tabular}

Table 5.5: The numbers of $e \mu$ events which passed the $e \mu$ event selection and the corresponding luminosities. The structure function is MRSDO-' and the Peterson parameter is set to 0.006 . 


\begin{tabular}{|c|c|c|c|c|c|}
\hline & & \multicolumn{2}{|c|}{ ISAJET } & \multicolumn{2}{|c|}{ ISALEP } \\
\hline & & $\begin{array}{c}\text { Number } \\
\text { of } \\
\text { events }\end{array}$ & $\begin{array}{l}\text { Luminosity } \\
\qquad\left(\mathrm{pb}^{-1}\right)\end{array}$ & $\begin{array}{c}\text { Number } \\
\text { of } \\
\text { events }\end{array}$ & $\begin{array}{l}\text { Luminosity } \\
\qquad\left(\mathrm{pb}^{-1}\right)\end{array}$ \\
\hline \multicolumn{2}{|l|}{ Process 1} & 926 & 0.789 & 49 & 0.323 \\
\hline \multirow{4}{*}{ Process 2} & $\bar{b} \rightarrow \bar{D}^{0} \rightarrow l$ & 24 & \multirow{4}{*}{0.601} & 2 & \multirow{4}{*}{0.325} \\
\hline & $\bar{b} \rightarrow D^{-} \rightarrow l$ & 9 & & 0 & \\
\hline & $\vec{b} \rightarrow D_{s}^{-} \rightarrow l$ & 2 & & 0 & \\
\hline & $\bar{b} \rightarrow$ baryon $_{\bar{c}} \rightarrow l$ & 3 & & 1 & \\
\hline \multirow{4}{*}{ Process 3} & $b \rightarrow D^{0} \rightarrow l$ & 25 & \multirow{4}{*}{0.641} & 2 & \multirow{4}{*}{0.225} \\
\hline & $b \rightarrow D^{+} \rightarrow l$ & 15 & & 3 & \\
\hline & $b \rightarrow D_{s}^{+} \rightarrow l$ & 3 & & 0 & \\
\hline & $b \rightarrow$ baryon $n_{c} \rightarrow l$ & 4 & & 0 & \\
\hline
\end{tabular}

Table 5.6: The numbers of $e \mu$ events which passed the $e \mu$ event selection and the corresponding luminosities. The structure function is MRSD0-' and the Peterson parameter is set to 0.009 . 


\begin{tabular}{|c|c|c|c|c|c|}
\hline & & \multicolumn{2}{|c|}{ ISAJET } & \multicolumn{2}{|c|}{ ISALEP } \\
\hline & & $\begin{array}{c}\text { Number } \\
\text { of } \\
\text { events }\end{array}$ & $\begin{array}{l}\text { Luminosity } \\
\qquad\left(\mathrm{pb}^{-1}\right)\end{array}$ & $\begin{array}{c}\text { Number } \\
\text { of } \\
\text { events }\end{array}$ & $\begin{array}{l}\text { Luminosity } \\
\qquad\left(\mathrm{pb}^{-1}\right)\end{array}$ \\
\hline \multicolumn{2}{|l|}{ Process 1} & 1169 & 0.589 & 203 & 0.586 \\
\hline \multirow{4}{*}{ Process 2} & $\bar{b} \rightarrow \bar{D}^{0} \rightarrow l$ & 34 & \multirow{4}{*}{0.627} & 14 & \multirow{4}{*}{0.586} \\
\hline & $\bar{b} \rightarrow D^{-} \rightarrow l$ & 21 & & 5 & \\
\hline & $\bar{b} \rightarrow D_{s}^{-} \rightarrow l$ & 9 & & 1 & \\
\hline & $\bar{b} \rightarrow$ baryon $_{\bar{c}} \rightarrow l$ & 3 & & 1 & \\
\hline \multirow{4}{*}{ Process 3} & $b \rightarrow D^{0} \rightarrow l$ & 34 & \multirow{4}{*}{0.512} & 9 & \multirow{4}{*}{0.586} \\
\hline & $b \rightarrow D^{+} \rightarrow l$ & 16 & & 1 & \\
\hline & $b \rightarrow D_{s}^{+} \rightarrow l$ & 2 & & 1 & \\
\hline & $b \rightarrow$ baryon $_{c} \rightarrow l$ & 11 & & 2 & \\
\hline
\end{tabular}

Table 5.7: The numbers of $e \mu$ events which passed the $e \mu$ event selection and the corresponding luminosities. The structure function is EHLQ and the Peterson parameter is set to 0.003 . 


\begin{tabular}{|c|c|c|c|c|c|}
\hline & & \multicolumn{2}{|c|}{ ISAJET } & \multicolumn{2}{|c|}{ ISALEP } \\
\hline & & $\begin{array}{c}\text { Number } \\
\text { of } \\
\text { events }\end{array}$ & $\begin{array}{l}\text { Luminosity } \\
\qquad\left(\mathrm{pb}^{-1}\right)\end{array}$ & $\begin{array}{c}\text { Number } \\
\text { of } \\
\text { events }\end{array}$ & $\begin{array}{l}\text { Luminosity } \\
\qquad\left(\mathrm{pb}^{-1}\right)\end{array}$ \\
\hline \multicolumn{2}{|l|}{ Process 1} & 1252 & 0.706 & 216 & 0.587 \\
\hline \multirow{4}{*}{ Process 2} & $\bar{b} \rightarrow \bar{D}^{0} \rightarrow l$ & 25 & \multirow{4}{*}{0.606} & 6 & \multirow{4}{*}{0.587} \\
\hline & $\bar{b} \rightarrow D^{-} \rightarrow l$ & 26 & & 5 & \\
\hline & $\bar{b} \rightarrow D_{s}^{-} \rightarrow l$ & 1 & & 0 & \\
\hline & $\bar{b} \rightarrow$ baryon $n_{\bar{c}} \rightarrow l$ & 5 & & 2 & \\
\hline \multirow{4}{*}{ Process 3} & $b \rightarrow D^{0} \rightarrow l$ & 45 & \multirow{4}{*}{0.563} & 6 & \multirow{4}{*}{0.587} \\
\hline & $b \rightarrow D^{+} \rightarrow \bar{l}$ & 25 & & $\overline{4}$ & \\
\hline & $b \rightarrow D_{s}^{+} \rightarrow l$ & 5 & & 1 & \\
\hline & $b \rightarrow$ baryon $_{c} \rightarrow l$ & 7 & & 1 & \\
\hline
\end{tabular}

Table 5.8: The numbers of $e \mu$ events which passed the $e \mu$ event selection and the corresponding luminosities. The structure function is EHLQ and the Peterson parameter is set to 0.006 . 


\begin{tabular}{|c|c|c|c|c|c|}
\hline & & \multicolumn{2}{|c|}{ ISAJET } & \multicolumn{2}{|c|}{ ISALEP } \\
\hline & & $\begin{array}{c}\text { Number } \\
\text { of } \\
\text { events }\end{array}$ & $\begin{array}{l}\text { Luminosity } \\
\qquad\left(\mathrm{pb}^{-1}\right)\end{array}$ & $\begin{array}{c}\text { Number } \\
\text { of } \\
\text { events }\end{array}$ & $\begin{array}{l}\text { Luminosity } \\
\qquad\left(\mathrm{pb}^{-1}\right)\end{array}$ \\
\hline \multicolumn{2}{|l|}{ Process 1} & 1129 & 0.705 & 204 & 0.585 \\
\hline \multirow{4}{*}{ Process 2} & $\bar{b} \rightarrow \bar{D}^{0} \rightarrow l$ & 39 & \multirow{4}{*}{0.705} & 8 & \multirow{4}{*}{0.585} \\
\hline & $\bar{b} \rightarrow D^{-} \rightarrow l$ & 34 & & 2 & \\
\hline & $\bar{b} \rightarrow D_{s}^{-} \rightarrow l$ & 7 & & 0 & \\
\hline & $\bar{b} \rightarrow$ baryon $_{\bar{c}} \rightarrow l$ & 3 & & 2 & \\
\hline \multirow{4}{*}{ Process 3} & $b \rightarrow D^{0} \rightarrow l$ & 39 & \multirow{4}{*}{0.705} & 5 & \multirow{4}{*}{0.557} \\
\hline & $b \rightarrow D^{+} \rightarrow l$ & 22 & & 5 & \\
\hline & $b \rightarrow D_{s}^{+} \rightarrow l$ & 4 & & 2 & \\
\hline & $b \rightarrow$ baryon $_{c} \rightarrow l$ & 6 & & 4 & \\
\hline
\end{tabular}

Table 5.9: The numbers of $e \mu$ events which passed the $e \mu$ event selection and the corresponding luminosities. The structure function is EHLQ and the Peterson parameter is set to 0.009 . 


\begin{tabular}{|c|c|c|c|c|c|}
\hline & & \multicolumn{2}{|c|}{ ISAJET } & \multicolumn{2}{|c|}{ ISALEP } \\
\hline & & $\begin{array}{c}\text { Number } \\
\text { of } \\
\text { events }\end{array}$ & $\begin{array}{l}\text { Luminosity } \\
\qquad\left(\mathrm{pb}^{-1}\right)\end{array}$ & $\begin{array}{c}\text { Number } \\
\text { of } \\
\text { events }\end{array}$ & $\begin{array}{l}\text { Luminosity } \\
\qquad\left(\mathrm{pb}^{-1}\right)\end{array}$ \\
\hline \multicolumn{2}{|l|}{ Process 1} & 1217 & 0.662 & 71 & 0.233 \\
\hline \multirow{4}{*}{ Process 2} & $\bar{b} \rightarrow \bar{D}^{0} \rightarrow l$ & 39 & \multirow{4}{*}{0.588} & 3 & \multirow{4}{*}{0.229} \\
\hline & $\bar{b} \rightarrow D^{-} \rightarrow l$ & 13 & & 2 & \\
\hline & $\bar{b} \rightarrow D_{s}^{-} \rightarrow l$ & 3 & & 0 & \\
\hline & $\bar{b} \rightarrow$ baryon $_{\bar{c}} \rightarrow l$ & 6 & & 1 & \\
\hline \multirow{4}{*}{ Process 3} & $b \rightarrow D^{0} \rightarrow l$ & 44 & \multirow{4}{*}{0.523} & 1 & \multirow{4}{*}{0.227} \\
\hline & $b \rightarrow D^{+} \rightarrow l$ & 18 & & 0 & \\
\hline & $b \rightarrow D_{s}^{+} \rightarrow l$ & 7 & & 0 & \\
\hline & $b \rightarrow$ baryon $_{c} \rightarrow l$ & 4 & & 0 & \\
\hline
\end{tabular}

Table 5.10: The numbers of $e \mu$ events which passed the $e \mu$ event selection and the corresponding luminosities. The structure function is DFLM and the Peterson parameter is set to 0.003 . 


\begin{tabular}{|c|c|c|c|c|c|}
\hline & & \multicolumn{2}{|c|}{ ISAJET } & \multicolumn{2}{|c|}{ ISALEP } \\
\hline & & $\begin{array}{c}\text { Number } \\
\text { of } \\
\text { events }\end{array}$ & $\begin{array}{l}\text { Luminosity } \\
\qquad\left(\mathrm{pb}^{-1}\right)\end{array}$ & $\begin{array}{c}\text { Number } \\
\text { of } \\
\text { events }\end{array}$ & $\begin{array}{l}\text { Luminosity } \\
\qquad\left(\mathrm{pb}^{-1}\right)\end{array}$ \\
\hline \multicolumn{2}{|l|}{ Process 1} & 995 & 0.586 & 57 & 0.233 \\
\hline \multirow{4}{*}{ Process 2} & $\bar{b} \rightarrow \bar{D}^{0} \rightarrow l$ & 27 & \multirow{4}{*}{0.485} & 2 & \multirow{4}{*}{0.218} \\
\hline & $\bar{b} \rightarrow D^{-} \rightarrow l$ & 14 & & 1 & \\
\hline & $\bar{b} \rightarrow D_{s}^{-} \rightarrow l$ & 2 & & 1 & \\
\hline & $\bar{b} \rightarrow$ baryon $_{\bar{c}} \rightarrow l$ & 3 & & 0 & \\
\hline \multirow{4}{*}{ Process 3} & $b \rightarrow D^{0} \rightarrow l$ & 39 & \multirow{4}{*}{0.596} & 2 & \multirow{4}{*}{0.215} \\
\hline & $b \rightarrow D^{+} \rightarrow l$ & $\overline{15}$ & & 0 & \\
\hline & $b \rightarrow D_{s}^{+} \rightarrow l$ & 2 & & 0 & \\
\hline & $b \rightarrow$ baryon $_{c} \rightarrow l$ & 9 & & 0 & \\
\hline
\end{tabular}

Table 5.11: The numbers of $e \mu$ events which passed the $e \mu$ event selection and the corresponding luminosities. The structure function is DFLM and the Peterson parameter is set to 0.006 . 


\begin{tabular}{|c|c|c|c|c|c|}
\hline & & \multicolumn{2}{|c|}{ ISAJET } & \multicolumn{2}{|c|}{ ISALEP } \\
\hline & & $\begin{array}{c}\text { Number } \\
\text { of } \\
\text { events }\end{array}$ & $\begin{array}{l}\text { Luminosity } \\
\qquad\left(\mathrm{pb}^{-1}\right)\end{array}$ & $\begin{array}{c}\text { Number } \\
\text { of } \\
\text { events }\end{array}$ & $\begin{array}{l}\text { Luminosity } \\
\qquad\left(\mathrm{pb}^{-1}\right)\end{array}$ \\
\hline \multicolumn{2}{|l|}{ Process 1} & 1071 & 0.663 & 40 & 0.199 \\
\hline \multirow{4}{*}{ Process 2} & $\bar{b} \rightarrow \bar{D}^{0} \rightarrow l$ & 30 & \multirow{4}{*}{0.663} & 1 & \multirow{4}{*}{0.198} \\
\hline & $\bar{b} \rightarrow D^{-} \rightarrow l$ & 21 & & 1 & \\
\hline & $\bar{b} \rightarrow D_{s}^{-} \rightarrow l$ & 6 & & 0 & \\
\hline & $\bar{b} \rightarrow$ baryon $_{\bar{c}} \rightarrow l$ & 7 & & 0 & \\
\hline \multirow{4}{*}{ Process 3} & $b \rightarrow D^{0} \rightarrow l$ & 34 & \multirow{4}{*}{0.663} & 2 & \multirow{4}{*}{0.199} \\
\hline & $b \rightarrow D^{+} \rightarrow l$ & 23 & & 1 & \\
\hline & $b \rightarrow D_{s}^{+} \rightarrow l$ & 5 & & 0 & \\
\hline & $b \rightarrow \overline{\text { baryon }} n_{c} \rightarrow l$ & 4 & & 0 & \\
\hline
\end{tabular}

Table 5.12: The numbers of $e \mu$ events which passed the $e \mu$ event selection and the corresponding luminosities. The structure function is DFLM and the Peterson parameter is set to 0.009 . 


\begin{tabular}{l||c|c}
\hline \hline Structure function & $f_{s}$ & Statistical uncertainty \\
\hline MRSD0-' & 0.1107 & 0.0084 \\
EHLQ & 0.1355 & 0.0050 \\
DFLM 260 & 0.1124 & 0.0084 \\
\hline \hline
\end{tabular}

Table 5.13: The $f_{s}$ for different structure functions where the Peterson parameter value is 0.006 .

ture function $[41,56]$, and Diemoz-Ferroni-Longo-Martinelli (DFLM) structure function $[54,57]$ with $\Lambda_{Q C D}=260 \mathrm{MeV}$. The $f_{s}$ values calculated for three different structure functions are shown in Table 5.13, where the default value of the Peterson parameter, 0.006 , was used. We take a center value of the minimum and the maximum values in Table 5.13: $f_{s}=0.123$.

\subsubsection{Correcting $f_{s}$ for $J / \psi$ and $b \rightarrow \bar{c} \rightarrow l$ events}

We correct $f_{s}$ for the contributions from other $b$-decay processes. Considering all of other possible decay processes, we have found that the following two processes have a sizable effect:

$$
\begin{array}{ll}
\text { Process } 5 & \text { Process } 6 \\
\left\{\begin{array}{lll}
b \rightarrow l^{-} \\
\bar{b} \rightarrow J / \psi \rightarrow l^{+}\left(\text {or } l^{-}\right)
\end{array}\right. & \begin{cases}b \rightarrow J / \psi \rightarrow l^{+}\left(\text {or } l^{-}\right) \\
\bar{b} \rightarrow l^{+}\end{cases}
\end{array}
$$

and

$$
\begin{aligned}
& \text { Process } 7 \quad \text { Process } 8 \\
& \left\{\begin{array} { l } 
{ b \rightarrow l ^ { - } } \\
{ \overline { b } \rightarrow \overline { c } W ; W \rightarrow c \overline { s } ; c \rightarrow l ^ { + } }
\end{array} \quad \left\{\begin{array}{l}
b \rightarrow c W ; W \rightarrow \bar{c} s ; \bar{c} \rightarrow l^{-} \\
\bar{b} \rightarrow l^{+}
\end{array}\right.\right.
\end{aligned}
$$

We call the first process $b \rightarrow J / \psi \rightarrow l$ events and the second $b \rightarrow \bar{c} \rightarrow l$ events. The $b \rightarrow J / \psi \rightarrow l$ events produce tri-leptons. Since we are rejecting events with three or more good leptons, the $b \rightarrow J / \psi \rightarrow l$ events come into our sample only when one of the two leptons from $J / \psi$ decays fails the selection cuts. Depending on which lepton 


\begin{tabular}{|l||c|c||c|c|}
\cline { 2 - 5 } \multicolumn{1}{c|}{} & \multicolumn{2}{|c||}{$\begin{array}{c}\text { Direct } b \bar{b} \text { production } \\
\text { (ISAJET) }\end{array}$} & \multicolumn{2}{c|}{$\begin{array}{c}\text { Gluon splitting } \\
\text { (ISALEP) }\end{array}$} \\
\cline { 2 - 5 } & $\begin{array}{c}\text { Number } \\
\text { of events }\end{array}$ & $\begin{array}{c}\text { Luminosity } \\
\left(\mathrm{pb}^{-1}\right)\end{array}$ & $\begin{array}{c}\text { Number } \\
\text { of events }\end{array}$ & $\begin{array}{c}\text { Luminosity } \\
\left(\mathrm{pb}^{-1}\right)\end{array}$ \\
\hline \hline$\left\{\begin{array}{l}b \rightarrow l^{-} \\
\bar{b} \rightarrow J / \psi \rightarrow l^{ \pm}\end{array}\right.$ & 195 & 0.720 & 12 & 0.295 \\
\hline$\left\{\begin{array}{l}b \rightarrow l^{-} \\
\bar{b} \rightarrow c \rightarrow l^{+}\end{array}\right.$ & 14 & 0.491 & 5 & 0.481 \\
\hline
\end{tabular}

Table 5.14: The numbers of $b \rightarrow J / \psi \rightarrow l$ and $b \rightarrow \bar{c} \rightarrow l$ events which passed the $e \mu$ event selection and the corresponding luminosities, where the structure function is MRSD0 ${ }^{\prime}$ and the Peterson parameter is set to 0.006 .

fails, $b \rightarrow J / \psi \rightarrow l$ events produce OS or LS pairs. On the other hand, $b \rightarrow \bar{c} \rightarrow l$ events contribute to OS. We generated these events in the same way as for the case of Processes 1 3. This time, $b$ mesons were forced to decay to leptons, and $\vec{b}$ mesons were forced to decay to $J / \psi$ for $b \rightarrow J / \psi \rightarrow l$ events, and to $c$ mesons for $b \rightarrow \bar{c} \rightarrow l$ events in the CLEOMC. The structure function was MRSD0 -' and the Peterson parameter was set to 0.006 . The number of Monte Carlo events that passed the $e \mu$ event selection, together with the integrated luminosities are summarized in Table 5.14. As in the case of Processes 1 3, the luminosities are not corrected for having forced $b$-decays to specific channels. The corresponding branching ratios used for the correction of the luminosities are $B r(b \rightarrow l+X)=0.110$ which is the same as before, and $B r(b \rightarrow J / \psi)=0.019$ and $B r(b \rightarrow \bar{c})=0.12$.

A summary of the numbers of events from Processes $1 \sim 3, b \rightarrow J / \psi \rightarrow l$ and $b \rightarrow \bar{c} \rightarrow$ $l$ events corresponding to the integrated luminosity of $20 \mathrm{pb}^{-1}$ is given in Table 5.15 . Note that $b \rightarrow J / \psi \rightarrow l$ events are considered to have an equal contribution to OS and LS. Processes $1 \sim 3$ gave $f_{s}=0.1107$ for MRSD0 ${ }^{\prime}$ as already shown in Table 5.13. 


\begin{tabular}{|c|c|c|c|}
\hline & & OS & LS \\
\hline$\left\{\begin{array}{l}b \rightarrow l^{-} \\
\bar{b} \rightarrow l^{+}\end{array}\right.$ & & $1630.0 \pm 124.3$ & - \\
\hline$\left\{\begin{array}{l}b \rightarrow l^{-} \\
\bar{b} \rightarrow \bar{c} \rightarrow l^{-}\end{array}\right.$ & $\left\{\begin{array}{l}b \rightarrow c \rightarrow l^{+} \\
\vec{b} \rightarrow l^{+}\end{array}\right.$ & - & $180.5 \pm 20.8$ \\
\hline$\left\{\begin{array}{l}b \rightarrow l^{-} \\
\bar{b} \rightarrow J / \psi \rightarrow l^{ \pm}\end{array}\right.$ & $\left\{\begin{array}{l}b \rightarrow J / \psi \rightarrow l^{ \pm} \\
\bar{b} \rightarrow l^{+}\end{array}\right.$ & $26.0 \pm 1.9$ & $26.0 \pm 1.9$ \\
\hline$\left\{\begin{array}{l}b \rightarrow l^{-} \\
\bar{b} \rightarrow c \rightarrow l^{+}\end{array}\right.$ & $\left\{\begin{array}{l}b \rightarrow \bar{c} \rightarrow l^{-} \\
\bar{b} \rightarrow l^{+}\end{array}\right.$ & $41.1 \pm 9.4$ & - \\
\hline
\end{tabular}

Table 5.15: The numbers of Monte Carlo events which passed the $e \mu$ selection for OS and LS at the integrated luminosity of $20 \mathrm{pb}^{-1}$. The structure function is MRSD0-' and Peterson parameter is set to 0.006 .

Inclusion of $b \rightarrow J / \psi \rightarrow l$ and $b \rightarrow \bar{c} \rightarrow l$ events changed $f_{s}$ to 0.1231 , which was a correction by a factor of $1.112 \pm 0.012$. The uncertainty is estimated from the statistics of $b \rightarrow J / \psi \rightarrow l$ and $b \rightarrow \bar{c} \rightarrow l$ Monte Carlo events. Multiplying 1.112 to 0.123 which was obtained only from Processes $1 \sim 3$ as the center value for the three different structure functions, we obtained $f_{s}=0.137$.

\subsubsection{Systematic uncertainties in $f_{s}$}

We discuss the uncertainties of $f_{s}$ in what follows. The relatively small contributions from $b \rightarrow J / \psi \rightarrow l$ and $b \rightarrow \bar{c} \rightarrow l$ events were not included in the Monte Carlo data used in estimation of this uncertainties.

First of all, our estimation of the uncertainty due to the structure function is already included in Table 5.13. The uncertainty due to $b$ quark fragmentation was estimated by changing the Peterson parameter, $\epsilon=0.006 \pm 0.003$ [7], by one sigma. The results for the MRSD0 ${ }^{\prime}$ structure function of are shown in Table 5.16. Figure 5.14 shows the $f_{s}$ 


\begin{tabular}{c||c|c}
\hline \hline Peterson parameter $(\epsilon)$ & $f_{s}$ & uncertainty \\
\hline 0.003 & 0.1055 & 0.0075 \\
0.006 & 0.1107 & 0.0084 \\
0.009 & 0.1190 & 0.0091 \\
\hline
\end{tabular}

Table 5.16: The $f_{s}$ for different values of the Peterson parameter $(\epsilon)$, where the structure function is MRSD0-'. The uncertainties are due to the statistics of the Monte Carlo events.

\begin{tabular}{l||c}
\hline \hline Fraction of gluon splitting & $f_{s}$ \\
\hline $0 \%$ & 0.1004 \\
$11.2 \%$ & 0.1107 \\
$22.4 \%$ & 0.1128 \\
\hline \hline
\end{tabular}

Table 5.17: The $f_{s}$ for different ratios of gluon splitting events to $b \bar{b}$ direct productions, where the structure function is $\mathrm{MRSD0}^{\prime}$ and the Peterson parameter is set to $\mathbf{0 . 0 0 6}$.

values for different structure functions and different values of the Peterson parameter. Due to the statistical uncertainty of the Monte Carlo events, we can not separate the uncertainties from the structure function and the fragmentation. Instead, we simply looked at the minimum and the maximum of center values of $f_{s}$ in Fig 5.14, which are $-14.3 \%$ and $12.9 \%$ of our $f_{s}$, respectively. We took $14.3 \%$ conservatively as the combined uncertainty of $f_{s}$ due to the statistics, the structure function and the fragmentation. The uncertainties due to the branching ratios, $\operatorname{Br}(b \rightarrow l+X)$ and $\operatorname{Br}(c \rightarrow l+X)$, were estimated to be $4.5 \%$ and $9.1 \%$, respectively, by propagating their uncertainties to $f_{s}$. We estimated a ratio of gluon splitting events to direct production events to be $11.2 \%$ from the Monte Carlo data where the structure function was MRSD0-' and the Peterson parameter was set to the default value of 0.006 . When we changed the ratio from $11.2 \%$ to $0 \%$ and $22.4 \%, f_{s}$ varied by $-9.3 \%$ and $+1.8 \%$, respectively, as shown in Table 5.17. We took $9.3 \%$ as the uncertainty due to the ratio of gluon splitting events to direct production events. The uncertainty due to the correction factor for $b \rightarrow J / \psi \rightarrow l$ 


\begin{tabular}{l|r}
\hline \hline Source & Uncertainties \\
\hline$\left\{\begin{array}{l}\text { Monte Carlo statistics } \\
\text { Fragmentation }\end{array}\right.$ & $14.3 \%$ \\
Structure function & \\
$B r(b \rightarrow l+X)$ & $4.5 \%$ \\
$B r(c \rightarrow l+X)$ & $9.1 \%$ \\
Fraction of gluon splitting & $9.3 \%$ \\
$J / \psi, b \rightarrow \bar{c} \rightarrow l$ & $1.1 \%$ \\
\hline Total & $19.9 \%$ \\
\hline \hline
\end{tabular}

Table 5.18: Uncertainties in $f_{s}$.

and $b \rightarrow \bar{c} \rightarrow l$ events was $1.1 \%$.

As a final result, we obtained

$$
f_{s}=0.137 \pm 0.027
$$

The uncertainties in $f_{s}$ are summarized in Table 5.18.

\subsection{Fraction of direct $c \bar{c}$ background}

\subsubsection{Method of $f_{c}$ estimation}

The fraction of direct $c \bar{c}$ background was estimated by fitting $P_{T}^{\text {rel }}$ distributions for $b \bar{b}$ Monte Carlo, $c \bar{c}$ Monte Carlo and fake leptons from the real data to the observed distribution. The $b \bar{b}$ Monte Carlo was already described in the previous section. The $c \bar{c}$ direct production events were generated by ISAJET with $P_{T}>2.9 \mathrm{GeV} / \mathrm{c}$ for both $c$ and $\bar{c}$, and $c \bar{c}$ events from gluon splitting were generated by ISALEP with $P_{T}>2.9 \mathrm{GeV} / \mathrm{c}$ for gluons. These events were processed by the QFL detector simulator.

We can rewrite $N^{e x p}(L S)$ and $N^{\exp }(O S)$ as

$$
N^{e x p}(L S)=2 \chi(1-\chi) N_{D}+\left[(1-\chi)^{2}+\chi^{2}\right] N_{S}+N_{f}(L S)
$$


and

$$
N^{e x p}(O S)=\left[(1-\chi)^{2}+\chi^{2}\right] N_{D}+2 \chi(1-\chi) N_{S}+N_{f}(O S)+N_{C}
$$

where $N_{D}, N_{S}, N_{C}$ and $N_{f}$ are the numbers of events for Process $1+5+6$, Process $2+3+5+6+7+8$, Process 4 in Tables 4.1 and 4.4 , and fake leptons. The difference between the numbers of OS and LS is written by

$$
N^{e x p}(O S)-N^{e x p}(L S)=(1-2 \chi)^{2}\left(N_{D}-N_{S}\right)+N_{C}+\Delta N_{f}
$$

where the last term, $\Delta N_{f} \equiv N_{f}(O S)-N_{f}(L S)$. There is a similar relation among $P_{T}^{\text {rel }}$ distributions,

$$
\begin{aligned}
& N^{e x p}(O S) H_{O S}^{e x p}\left(P_{T}^{\text {rel }}\right)-N^{e x p}(L S) H_{L S}^{\exp }\left(P_{T}^{\text {rel }}\right)= \\
& \quad(1-2 \chi)^{2}\left[N_{D} H_{d}\left(P_{T}^{\text {rel }}\right)-N_{S} H_{s}\left(P_{T}^{\text {rel }}\right)\right]+N_{C} H_{c}\left(P_{T}^{\text {rel }}\right)+\Delta N_{f} H_{f}\left(P_{T}^{\text {rel }}\right),
\end{aligned}
$$

where $H\left(P_{T}^{\text {rel }}\right)$ represents the $P_{T}^{\text {rel }}$ distribution function and is normalized to be one. This relation holds for muons and electrons separately. We fitted

$$
n_{b}\left(H_{d}\left(P_{T}^{r e l}\right)-\frac{N_{S}}{N_{D}} H_{s}\left(P_{T}^{r e l}\right)\right)+n_{c} H_{c}\left(P_{T}^{r e l}\right)+\Delta N_{f} H_{f}\left(P_{T}^{r e l}\right)
$$

to

$$
H_{O S}^{e x p}\left(P_{T}^{r e l}\right)-H_{L S}^{e x p}\left(P_{T}^{r e l}\right)
$$

taking $n_{b}$ and $n_{c}$ are fitting parameters and setting $\Delta N_{f}$ to the observed value. Since we defined Eq. (5.27) for muons and electrons separately, the $\Delta N_{f}$ can be defined to be a difference of number of fake leptons. Then the $\Delta N_{f}$ is calculated to be $70 \pm 23$ and $29 \pm 77$ for fake muons and fake electrons, respectively, from Table 5.2 in Section 5.1.

The $P_{T}^{\text {rel }}$ distributions for $b \bar{b}$ and $c \bar{c}$ were obtained from the Monte Carlo's. As fake muons, we used muon candidates which had a stub only in CMU chambers within the CMU and CMP fiducial region. As fake electrons, we used electron candidates with 
$\triangle X(C E S)>6 \mathrm{~cm}$ which were considered as photons overlapped by charged hadrons in the EM calorimeter. We here assume that the $P_{T}^{\text {rel }}$ distribution for photons overlapped by charged hadrons and that for other sources of fake electrons are the same. One should note that the magnitude of $\Delta N_{f}$ for fake electrons itself is small compared to the total number of the fitted distribution, that is $N^{\exp }(O S)-N^{\exp }(L S)=849$ derived from Table 5.2. The result, therefor, does not significantly depend on it. The number of direct $c \bar{c}$ background is given by $N_{C}=n_{c} \int H_{c} d P_{T}^{\text {rel }}$ in the region of $P_{T}^{\text {rel }}>1.5 \mathrm{GeV} / \mathrm{c}$. The fraction of direct $c \bar{c}$ background is obtained by

$$
f_{c}=\frac{N_{C}}{N_{D}}=\frac{N_{C}}{\left[N^{\exp }(O S)\left(1-F_{e \mu}(O S)\right)+N^{\exp }(L S)\left(1-F_{e \mu}(L S)\right)-N_{C}\right] \cdot \frac{1}{1+f_{\bullet}}} .
$$

\subsubsection{Estimation of $f_{c}$}

The fitted $P_{T}^{\text {rel }}$ distributions for muons and electrons are shown in Fig. 5.15, where the structure function is used MRSD0 ${ }^{\prime}$ In Fig. 5.15 (a) ((b)), we did not apply the $P_{T}^{\text {rel }}$ cut to muons (electrons) while $P_{T}^{\text {rel }}$ of electrons (muons) was required to be greater than $1.5 \mathrm{GeV} / \mathrm{c}$. The shape difference between the $P_{T}^{\text {rel }}$ distributions for muons and electrons comes from the different lepton $P_{T}$ thresholds, which was confirmed by raising up the $P_{T}$ threshold for muons. The number of direct $c \bar{c}$ background $N_{C}$ was estimated to be $-6 \pm 26$ from the fit of electron $P_{T}^{r e l}$ distribution and $34 \pm 18$ from muon $P_{T}^{\text {rel }}$. These numbers are consistent with each other within the statistical uncertainties. The weighted average of the numbers, $21 \pm 15$, was used in this analysis.

We estimated $f_{c}$ for two other structure functions as in the case of $f_{s}$. The fitted $P_{T}^{\text {rel }}$ distributions for EHLQ and DFLM structure functions are shown in Figs. 5.16 and 5.17, respectively. The results for three structure functions are shown in Table 5.19. We take as $f_{c}$ the center value of the minimum and the maximum values in Table 5.19, which is 0.007 . 


\begin{tabular}{l||c|c}
\hline \hline Structure function & $f_{c}$ & Statistical uncertainty \\
\hline MRSD0 ${ }^{\prime}$ & 0.013 & 0.010 \\
EHLQ & 0.009 & 0.010 \\
DFLM 260 & 0.001 & 0.012 \\
\hline \hline
\end{tabular}

Table 5.19: The $f_{c}$ for different structure functions.

\begin{tabular}{l|l|r}
\hline \hline \multicolumn{2}{|c|}{ Difference between the numbers of fake } & \multirow{2}{*}{$f_{c}$} \\
leptons in OS and LS & \\
\hline Fake muon & Fake electron & \\
\hline 70 & 29 & $\mathbf{0 . 0 1 3}$ \\
$70+23$ & $29+77$ & -0.002 \\
$70-23$ & $29-77$ & 0.031 \\
\hline \hline
\end{tabular}

Table 5.20: The $f_{c}$ for values of difference between the numbers of fake leptons in OS and LS.

\subsubsection{Systematic uncertainties in $f_{c}$}

The uncertainty due to the structure function was obtained in the same way as for $f_{s}$. The combined uncertainty in $f_{c}$ due to statistics and the structure function is taken to be $85.7 \%$ which is half of the difference between the minimum and the maximum values in Table 5.19.

The uncertainty in $f_{c}$ caused by the uncertainty in $n_{f}$ was estimated by changing the $n_{f}$ by one sigma around the center value of $n_{f}$ in the fitting. The default structure function, MRSD0-', was used for the Monte Carlo data. From Table 5.20 showing the results, we estimated the uncertainty to be $139.6 \%$, where we took the largest deviation from the center value divided by the center value.

Finally, we got

$$
f_{c}=0.007 \pm 0.011
$$

The uncertainties in $f_{c}$ are summarized in Table 5.21 


\begin{tabular}{l|r}
\hline \hline Source & Uncertainties \\
\hline Statistics & $85.7 \%$ \\
Structure function & $139.6 \%$ \\
Fake leptons & $163.4 \%$ \\
\hline Total & \\
\hline \hline
\end{tabular}

Table 5.21: Uncertainties in $f_{c}$. 


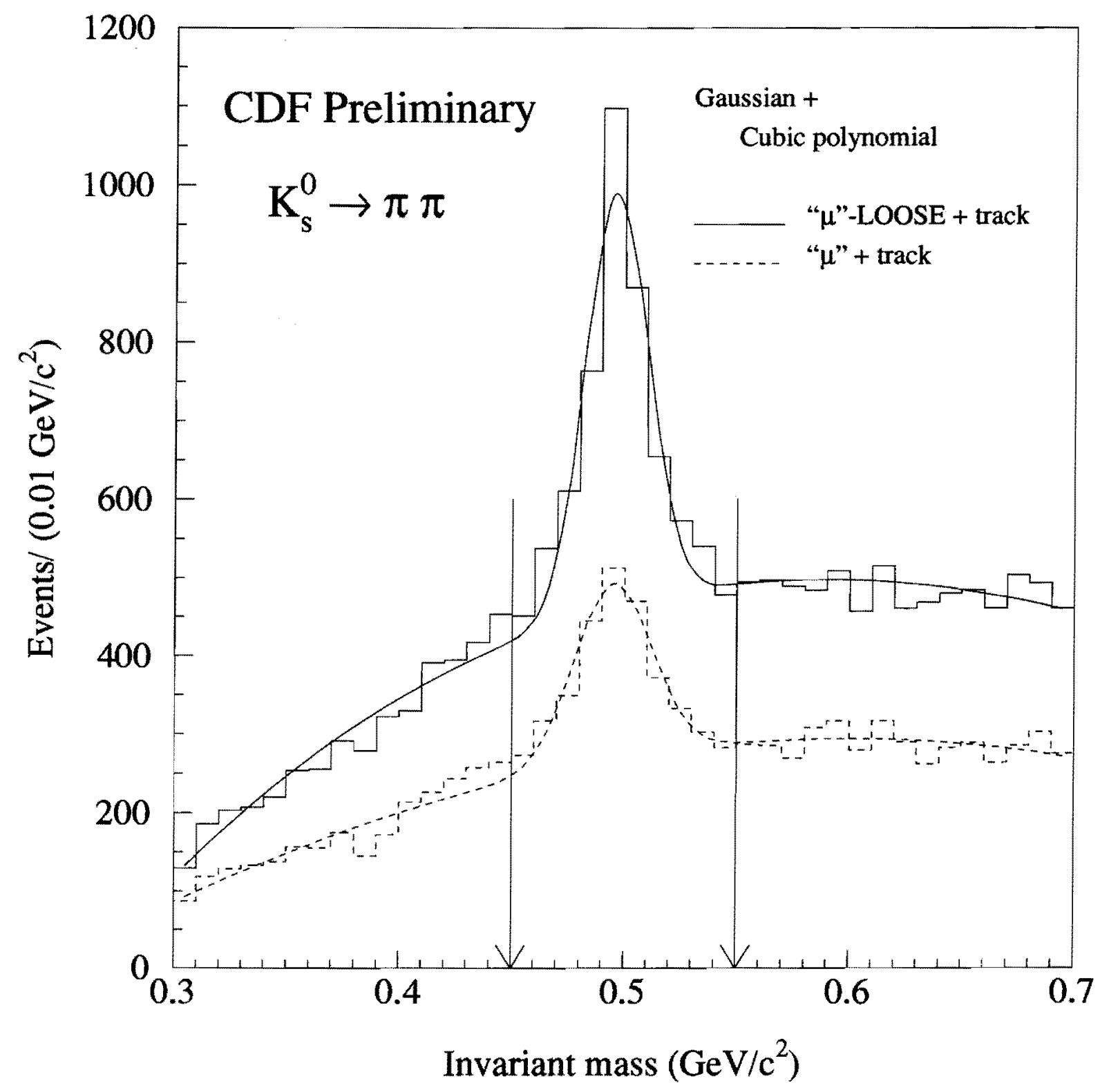

Figure 5.1: Invariant mass of a muon candidate track and an oppositely charged hadron track with a $\pi-\pi$ mass assignment. The dashed (solid) histogram is for the case where the muon candidate track passed the muon identification cut (the loose cut). 


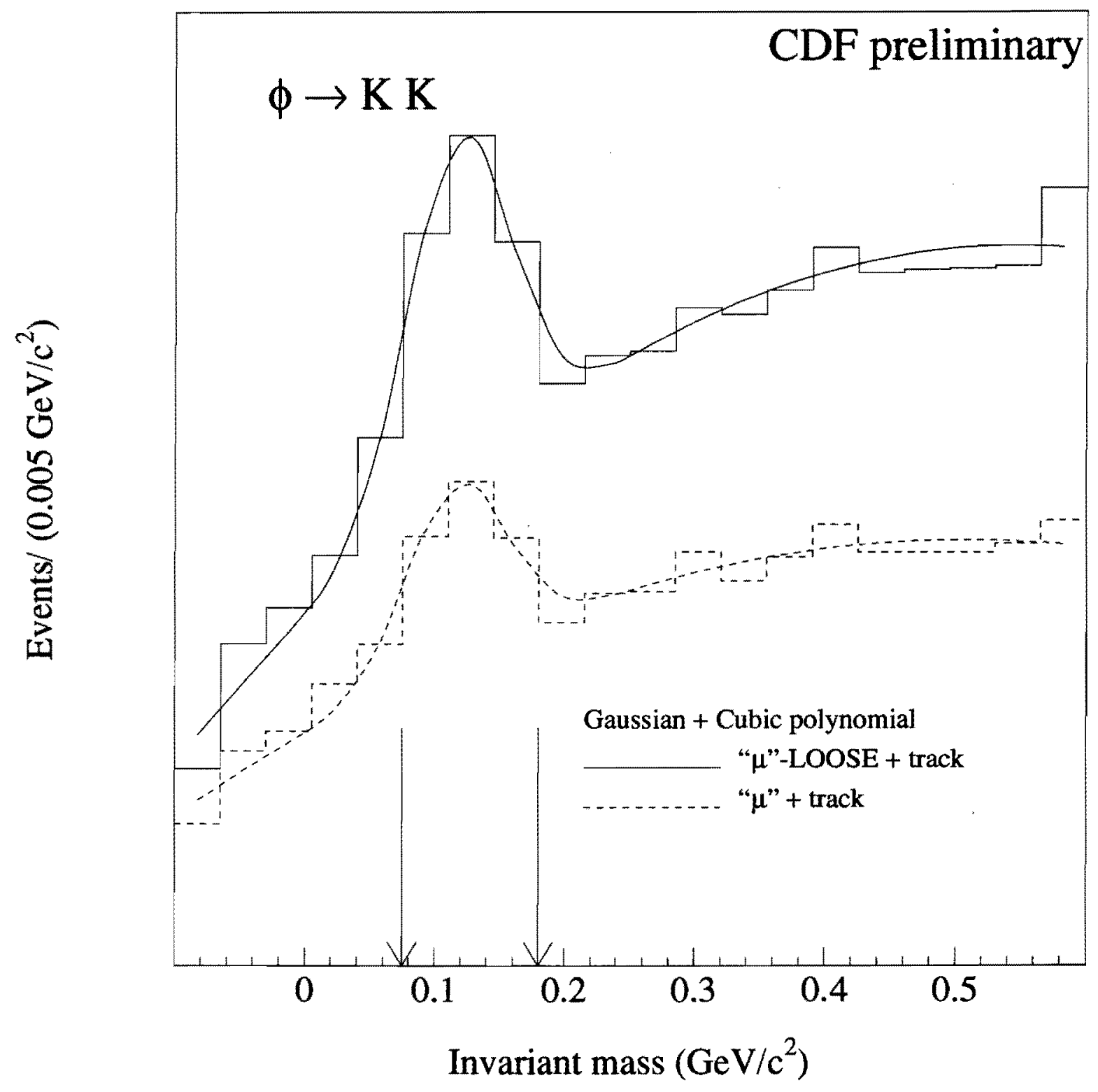

Figure 5.2: Invariant mass of a muon candidate track and an oppositely charged hadron track with a $K-K$ mass assignment. The dashed (solid) histogram is for the case where the muon candidate track passed the muon identification cut (the loose cut). 

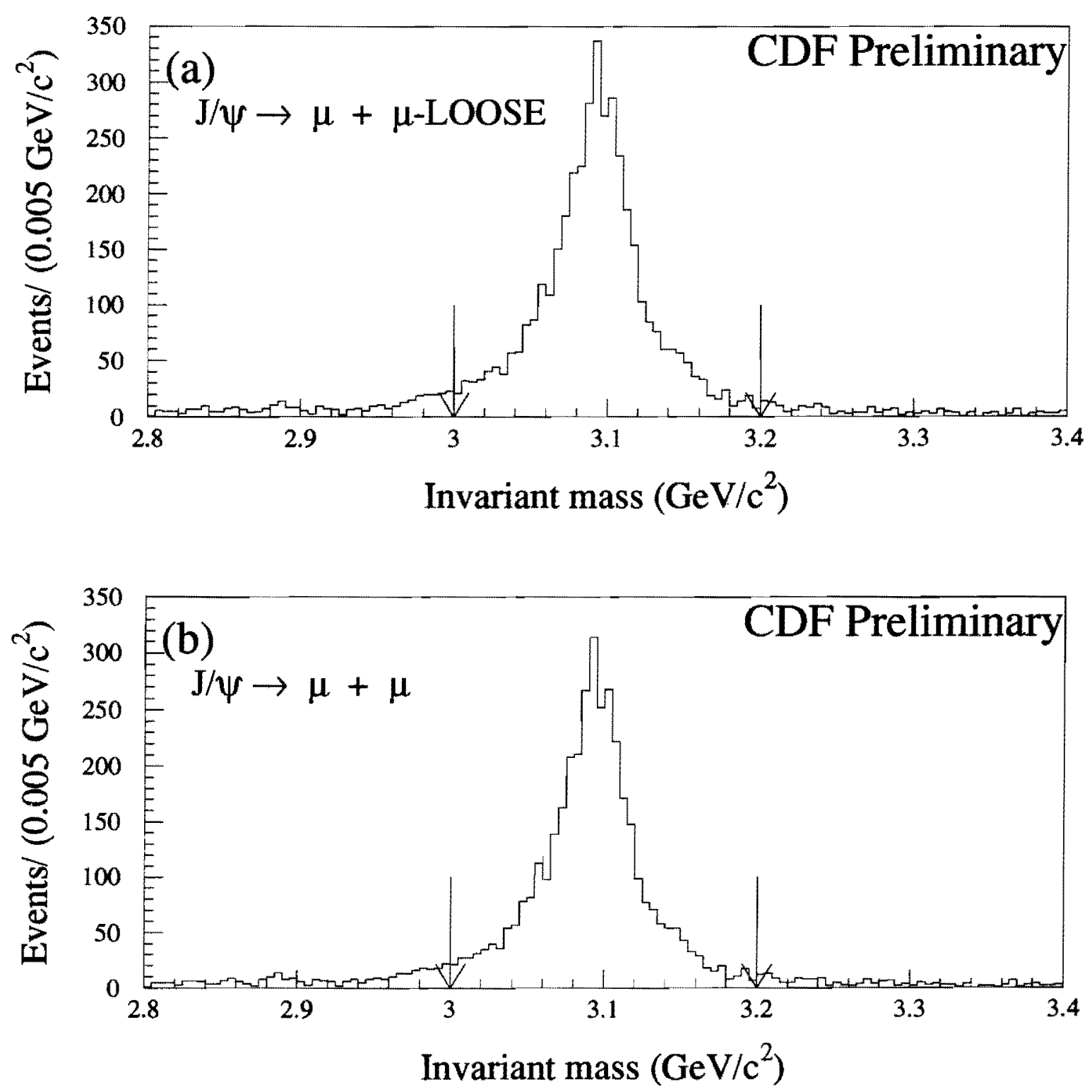

Figure 5.3: Invariant mass distributions for dimuons. (a) One of the two muons passed the muon identification cut and the other muon passed the loose one. (b) Both of the two muons passed the muon identification cut. 

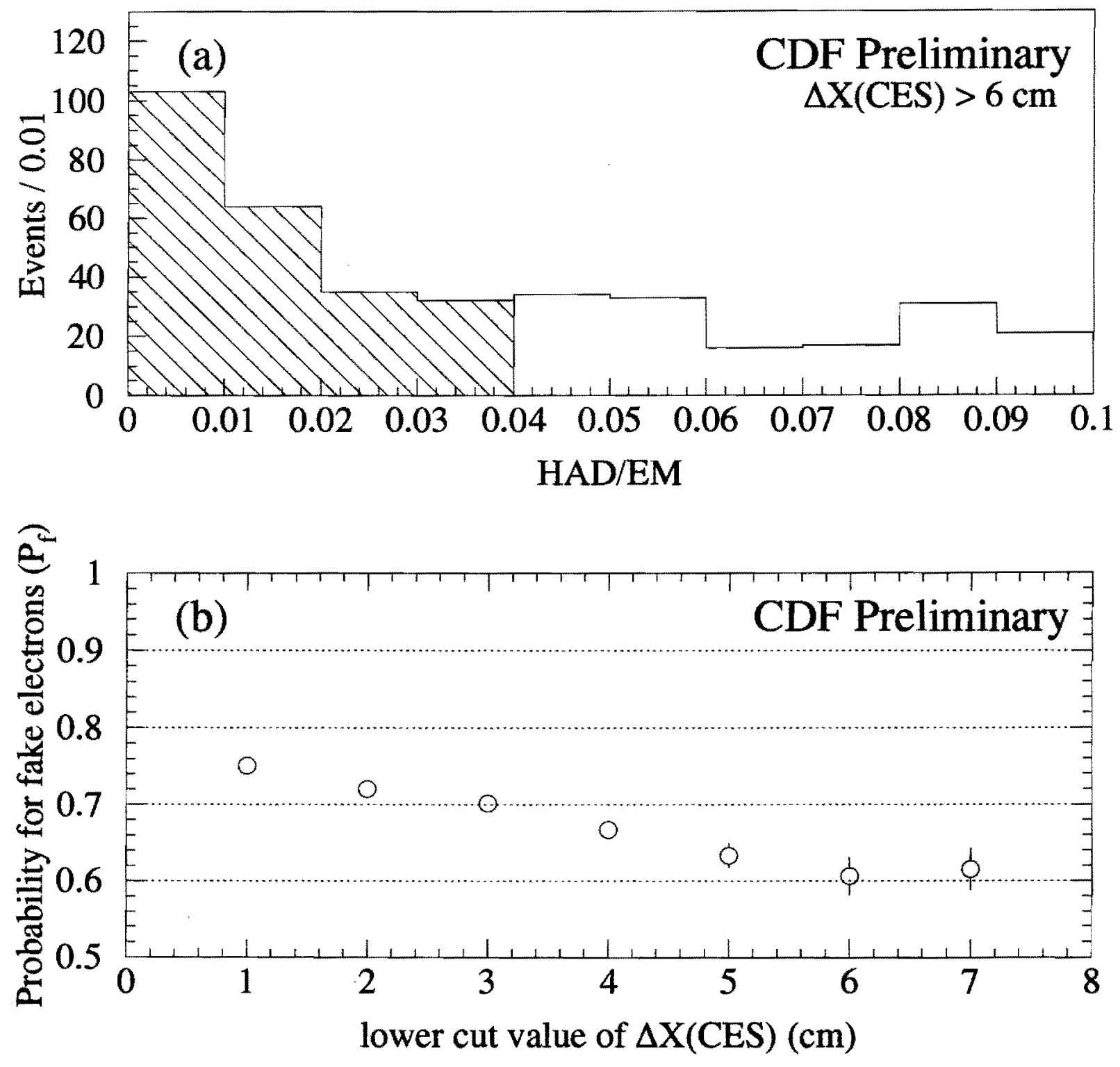

Figure 5.4: (a) $H A D / E M$ distribution for electron candidates with $\triangle X(C E S)>6$ $\mathrm{cm}$. Electron candidates in the hatched area pass the electron identification cut except $\triangle X(C E S)$ cut. (b) Probabilities for fake electrons (photon + charged hadron overlap) as a function of the lower cut value of $\triangle X(C E S)$. 

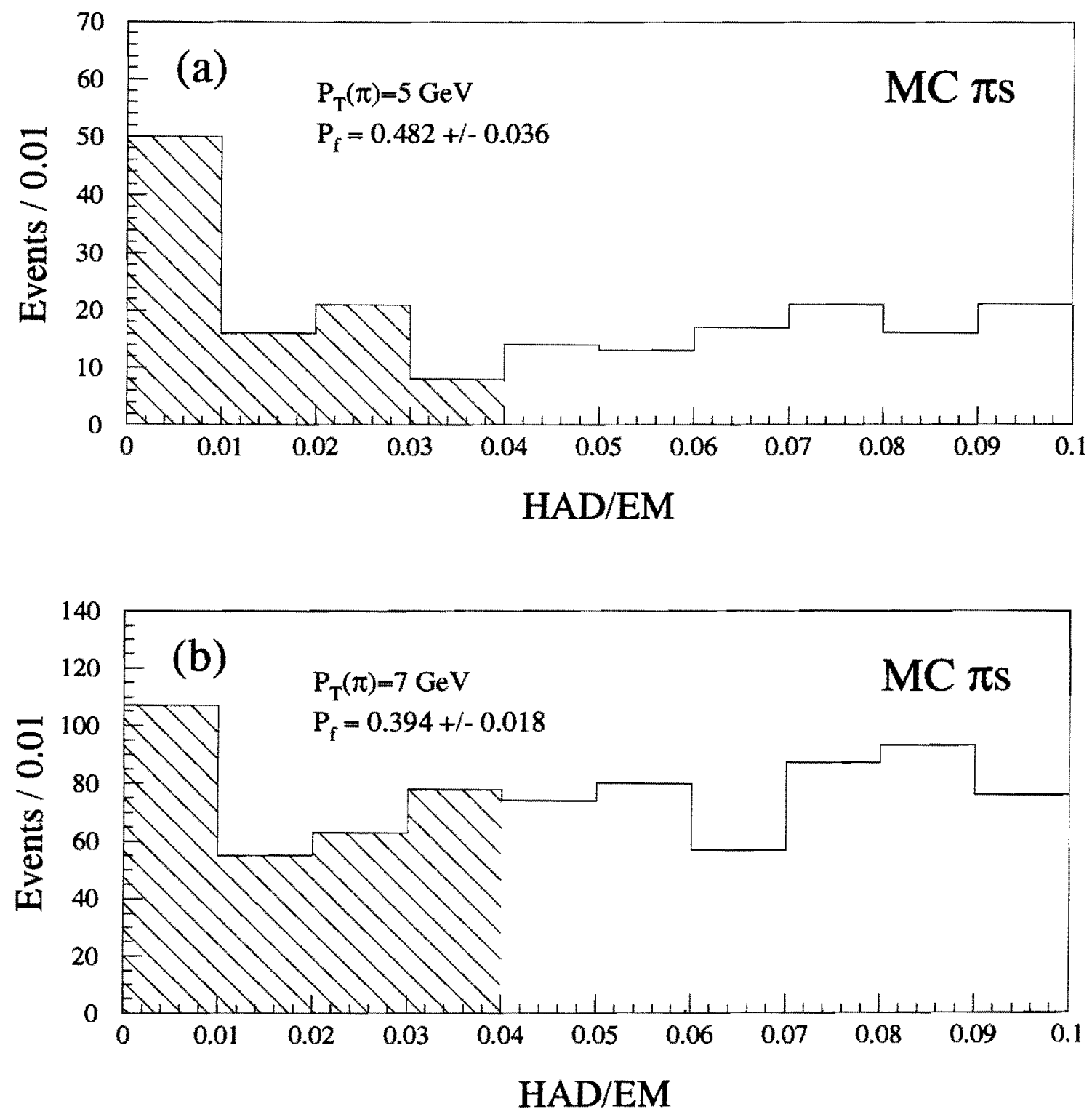

Figure 5.5: $H A D / E M$ distributions for generated pions passing the loose electron identification cut for (a) $P_{T}=5 \mathrm{GeV} / \mathrm{c}$ and (b) $P_{T}=7 \mathrm{GeV} / \mathrm{c}$. Pions in the hatched area pass the electron identification cut. 

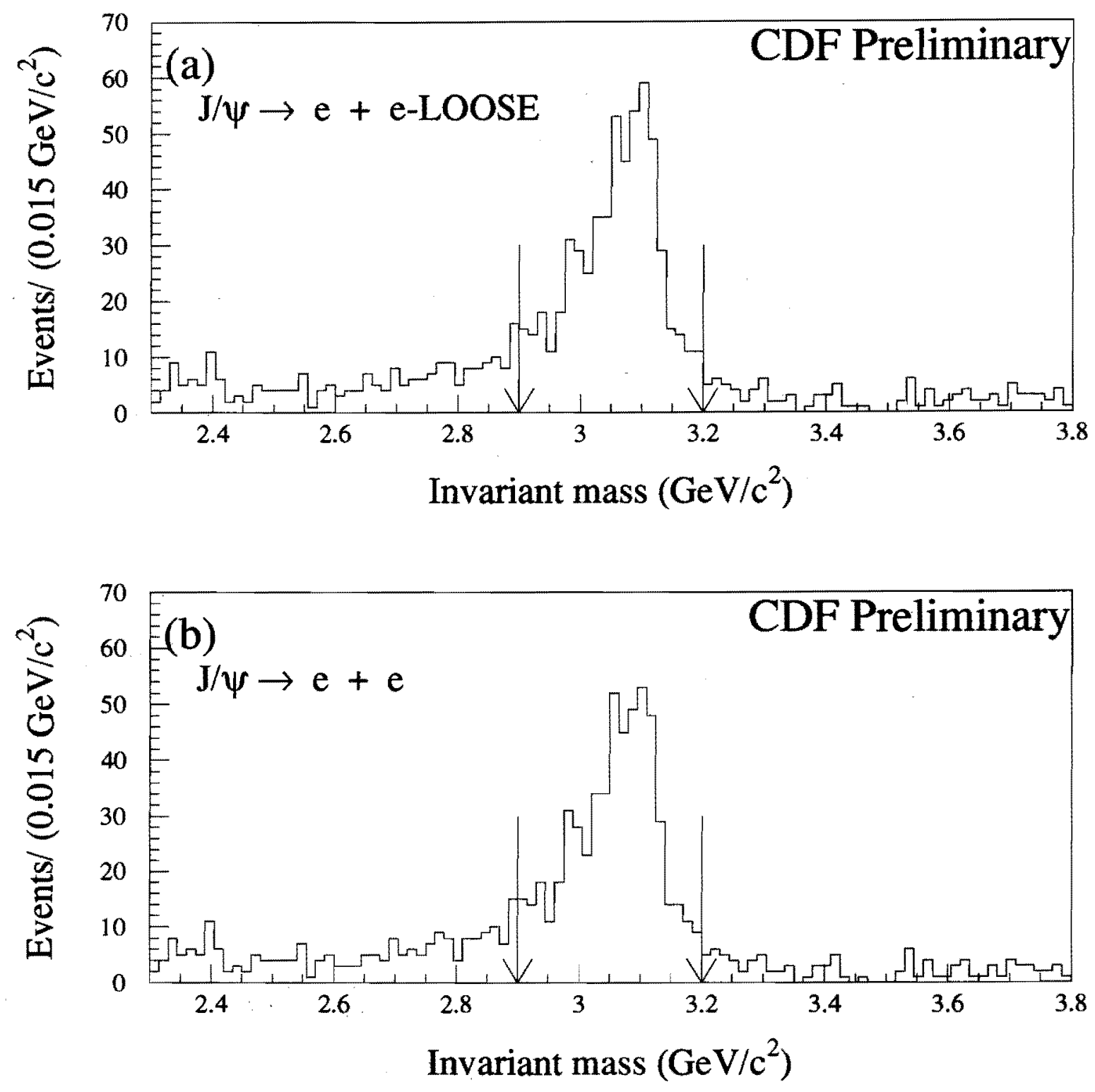

Figure 5.6: Invariant mass distributions for dielectrons. (a) One of the two electrons passed the electron identification selection and the other electron passed the loose one. (b) Both of the two electrons passed the electron identification selection. 


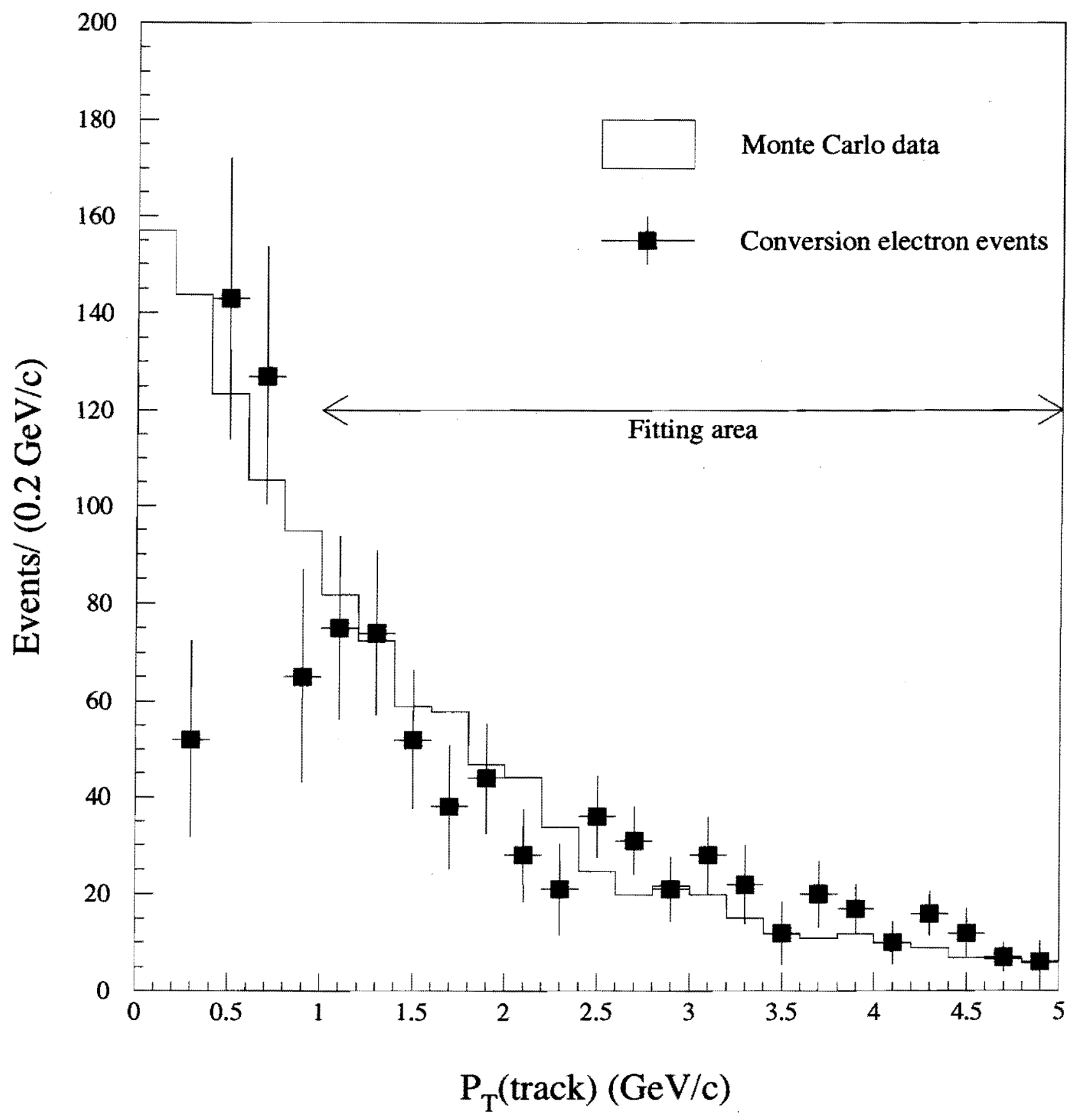

Figure 5.7: Transverse momentum distributions of electrons for conversion electron sample and the Monte Carlo data. The Monte Carlo data is normalized in a region of $1<P_{T}($ track $)<5 \mathrm{GeV} / \mathrm{c}$. 


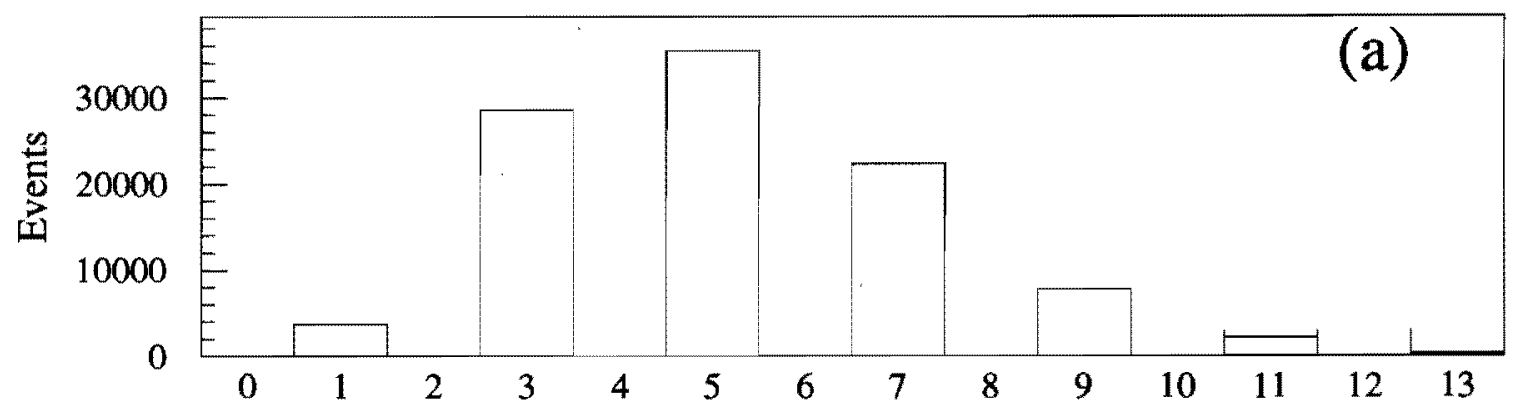

Number of charged particles produced from $\mathrm{B}_{\mathrm{u}}^{-}$

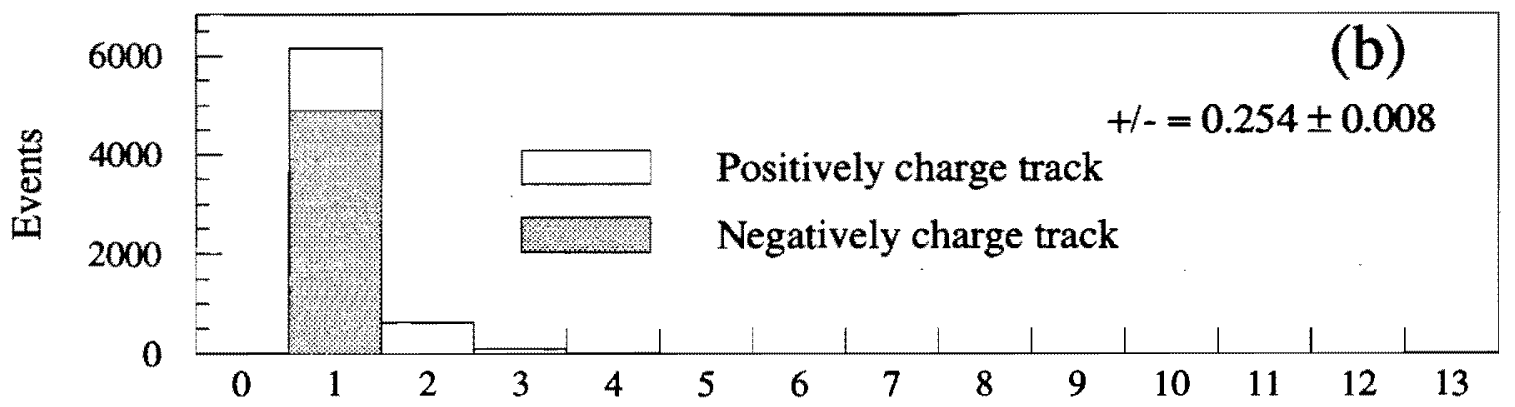

Number of charged tracks from $\mathrm{B}_{\mathrm{u}}^{-} ; \mathrm{P}_{\mathrm{T}}$ (track) $>3 \mathrm{GeV} / \mathrm{c}, \mid \eta$ (track) $) \mid<0.6$

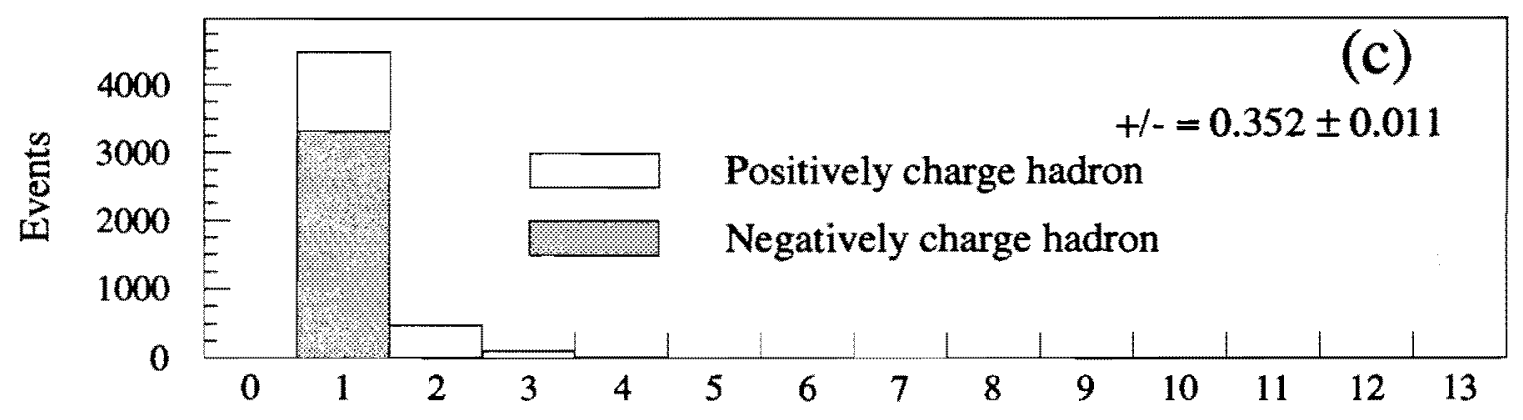

Number of charged hadrons from $\mathrm{B}_{\mathrm{u}}^{-} ; \mathrm{P}_{\mathrm{T}}($ hadron) $>3 \mathrm{GeV} / \mathrm{c}$, $\mid \eta$ (hadron) $\mid<0.6$

Figure 5.8: Charged particle multiplicities from the $B_{u}^{-}$meson. (a) Multiplicity of charged particles produced from $B_{u}^{-}$mesons. (b) Multiplicity of charged tracks (hadron or lepton) with $P_{T}>3 \mathrm{GeV} / \mathrm{c}$ and $|\eta|<0.6$. (c) Multiplicity of charged hadrons with $P_{T}>3 \mathrm{GeV} / \mathrm{c}$ and $|\eta|<0.6$. The leptons are removed in this distribution. The hatched area in multiplicity one bin in (b) and (c) represents negatively charged tracks and hadrons. 


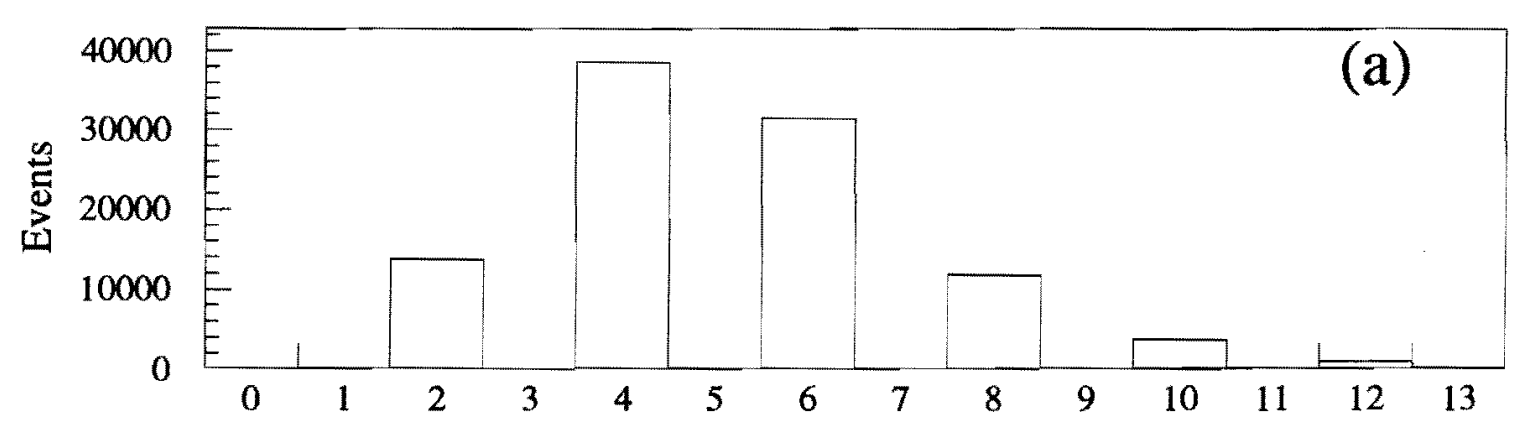

Number of charged particles produced from $\overline{\mathrm{B}}_{\mathrm{d}}^{0}$

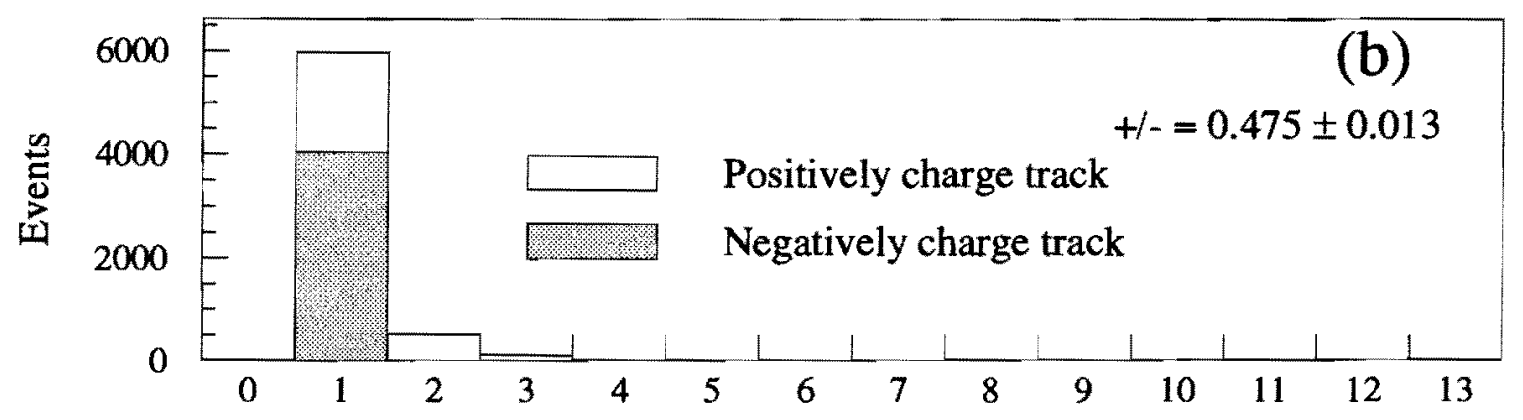

Number of charged tracks from $\overline{\mathrm{B}}_{\mathrm{d}}^{0} ; \mathrm{P}_{\mathrm{T}}$ (track) $>3 \mathrm{GeV} / \mathrm{c}, \mid$ (track) $\mid<0.6$

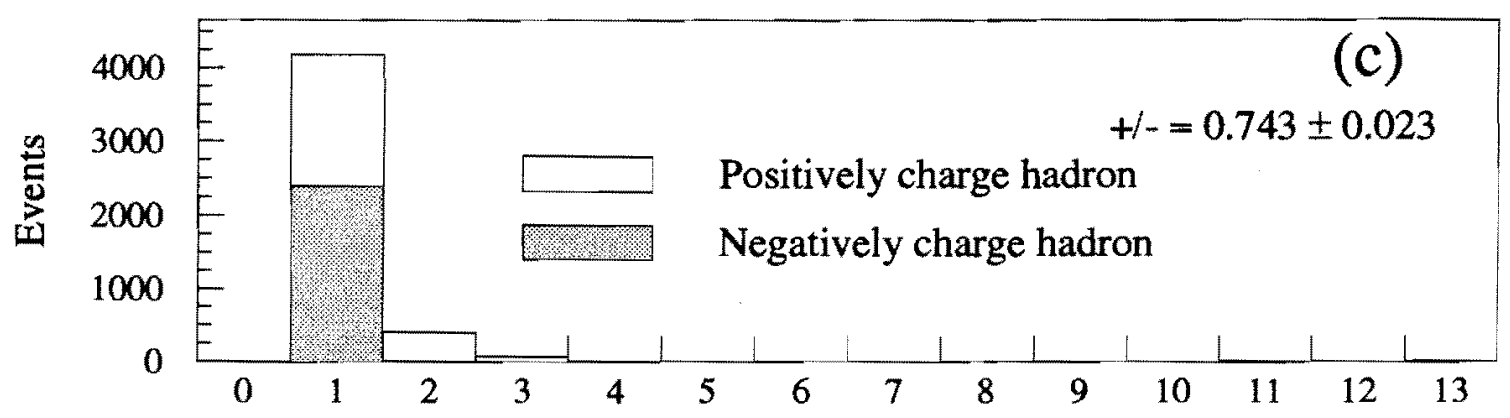

Number of charged hadrons from $\overline{\mathrm{B}}_{\mathrm{d}}^{0} ; \mathrm{P}_{\mathrm{T}}$ (hadron) $>3 \mathrm{GeV} / \mathrm{c}$, $\mid$ (hadron) $\mid<0.6$

Figure 5.9: Charged particle multiplicities from the $\bar{B}_{d}^{0}$ meson. (a) Multiplicity of charged particles produced from $\bar{B}_{d}^{0}$ mesons. (b) Multiplicity of charged tracks (hadron or lepton) with $P_{T}>3 \mathrm{GeV} / \mathrm{c}$ and $|\eta|<0.6$. (c) Multiplicity of charged hadrons with $P_{T}>3 \mathrm{GeV} / \mathrm{c}$ and $|\eta|<0.6$. The leptons are removed in this distribution. The hatched area in multiplicity one bin in (b) and (c) represents negatively charged tracks and hadrons. 


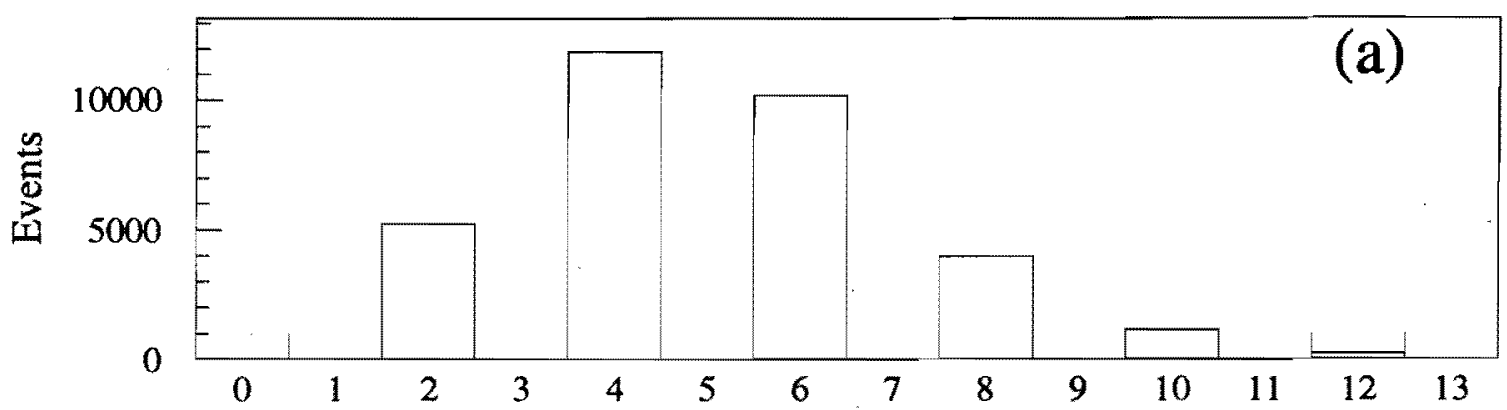

Number of charged particles produced from $\overline{\mathrm{B}}_{\mathrm{s}}^{0}$

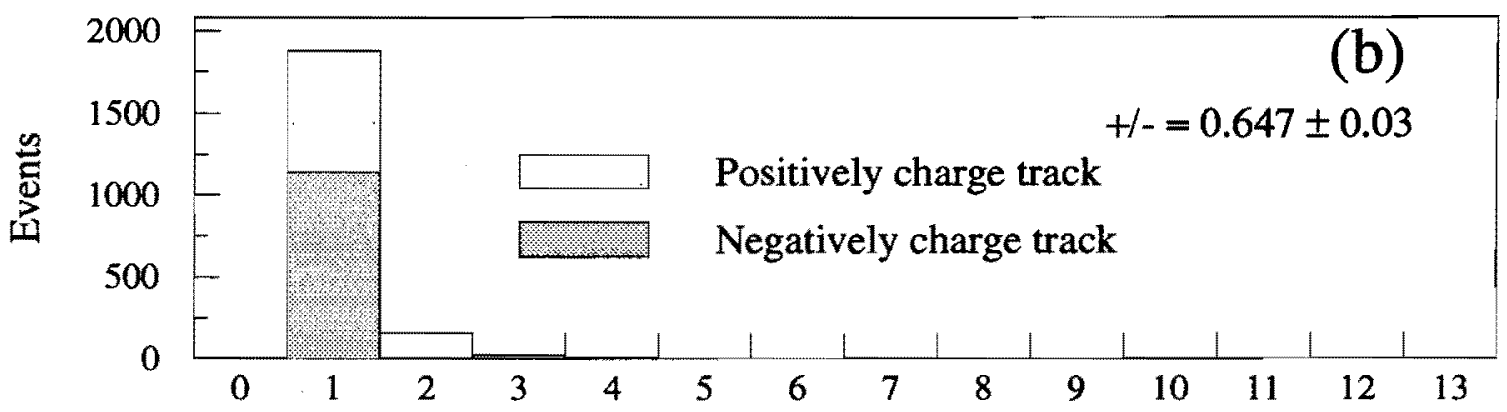

Number of charged tracks from $\overline{\mathrm{B}}_{\mathrm{s}}^{0} ; \mathrm{P}_{\mathrm{T}}$ (track) $>3 \mathrm{GeV} / \mathrm{c}$, $\mid$ (track) $\mid<0.6$

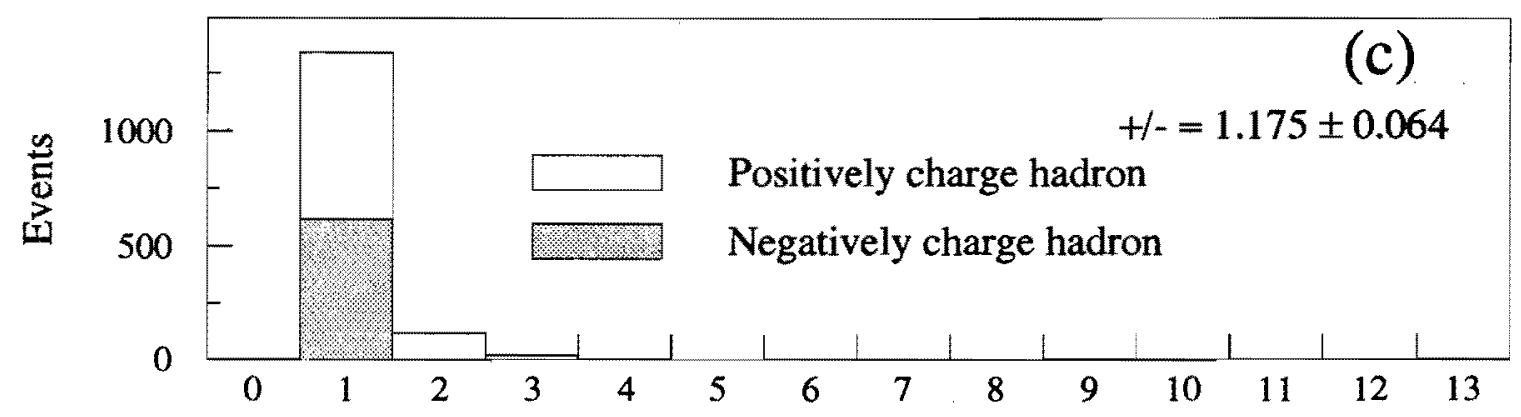

Number of charged hadrons from $\overline{\mathrm{B}}_{\mathrm{s}}^{0} ; \mathrm{P}_{\mathrm{T}}$ (hadron) $>3 \mathrm{GeV} / \mathrm{c}$, $\mid \eta$ (hadron) $\mid<0.6$

Figure 5.10: Charged particle multiplicities from the $\bar{B}_{s}^{0}$ meson. (a) Multiplicity of charged particles produced from $\bar{B}_{s}^{0}$ mesons. (b) Multiplicity of charged tracks (hadron or lepton) with $P_{T}>3 \mathrm{GeV} / \mathrm{c}$ and $|\eta|<0.6$. (c) Multiplicity of charged hadrons with $P_{T}>3 \mathrm{GeV} / \mathrm{c}$ and $|\eta|<0.6$. The leptons are removed in this distribution. The hatched area in multiplicity one bin in (b) and (c) represents negatively charged tracks and hadrons. 


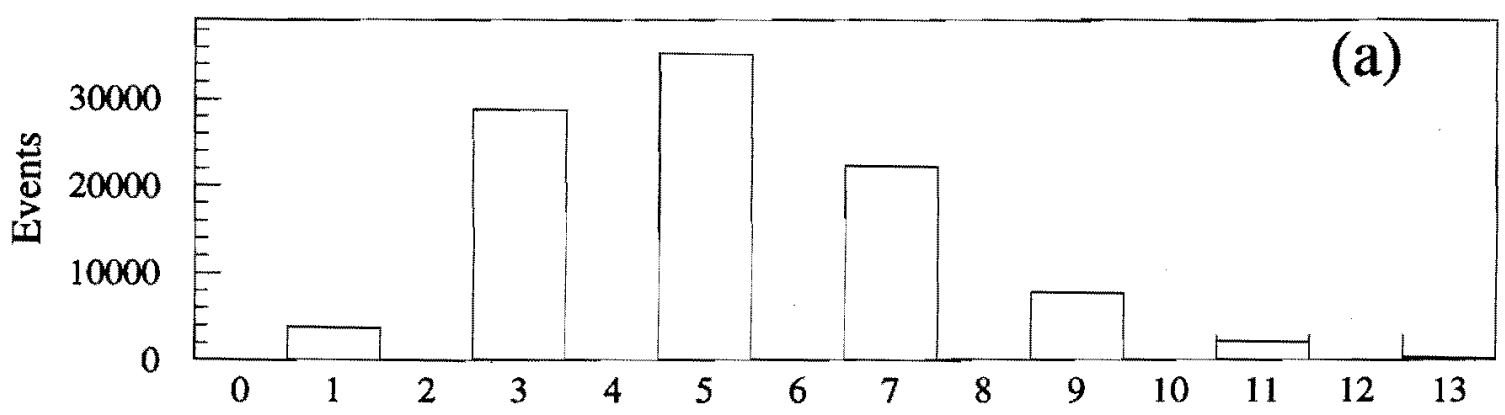

Number of charged particles produced from $\mathrm{B}_{\mathrm{u}}^{+}$

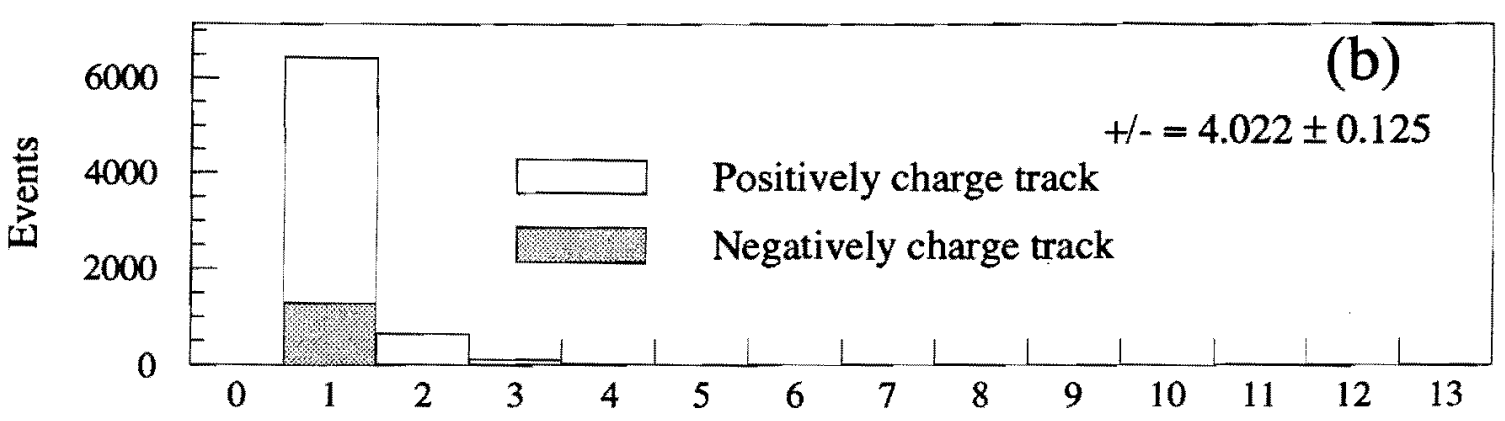

Number of charged tracks from $\mathrm{B}_{\mathrm{u}}^{+} ; \mathrm{P}_{\mathrm{T}}$ (track) $>3 \mathrm{GeV} / \mathrm{c}, \mid \eta$ (track) $\mid<0.6$

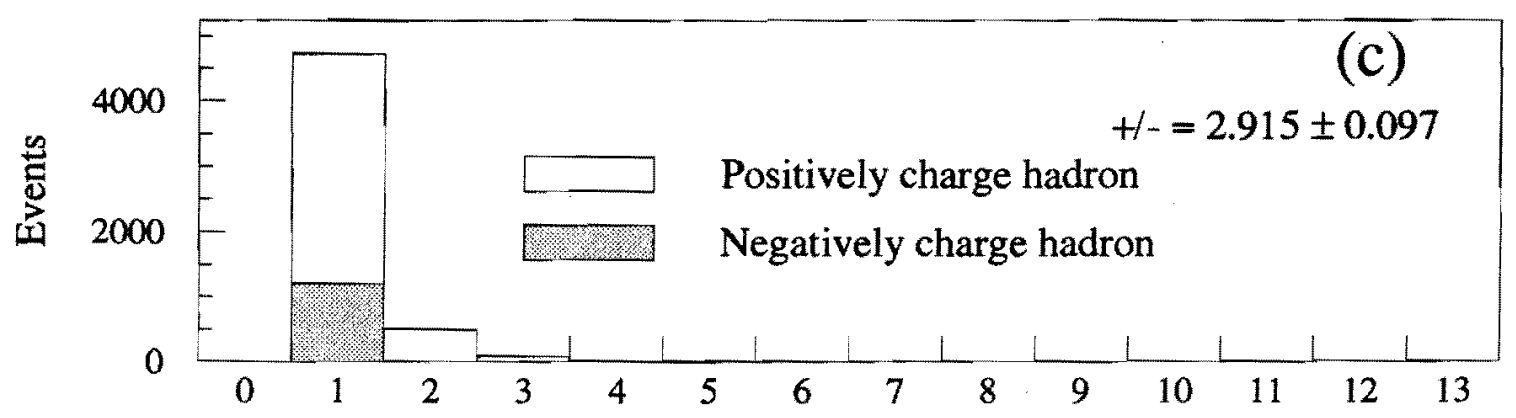

Number of charged hadrons from $\mathrm{B}_{\mathrm{u}}^{+} ; \mathrm{P}_{\mathrm{T}}$ (hadron) $>3 \mathrm{GeV} / \mathrm{c}$, $\mid$ (hadron) $\mid<0.6$

Figure 5.11: Charged particle multiplicities from the $B_{u}^{+}$meson. (a) Multiplicity of charged particles produced from $B_{u}^{+}$mesons. (b) Multiplicity of charged tracks (hadron or lepton) with $P_{T}>3 \mathrm{GeV} / \mathrm{c}$ and $|\eta|<0.6$. (c) Multiplicity of charged hadrons with $P_{T}>3 \mathrm{GeV} / \mathrm{c}$ and $|\eta|<0.6$. The leptons are removed in this distribution. The hatched area in multiplicity one bin in (b) and (c) represents negatively charged tracks and hadrons. 

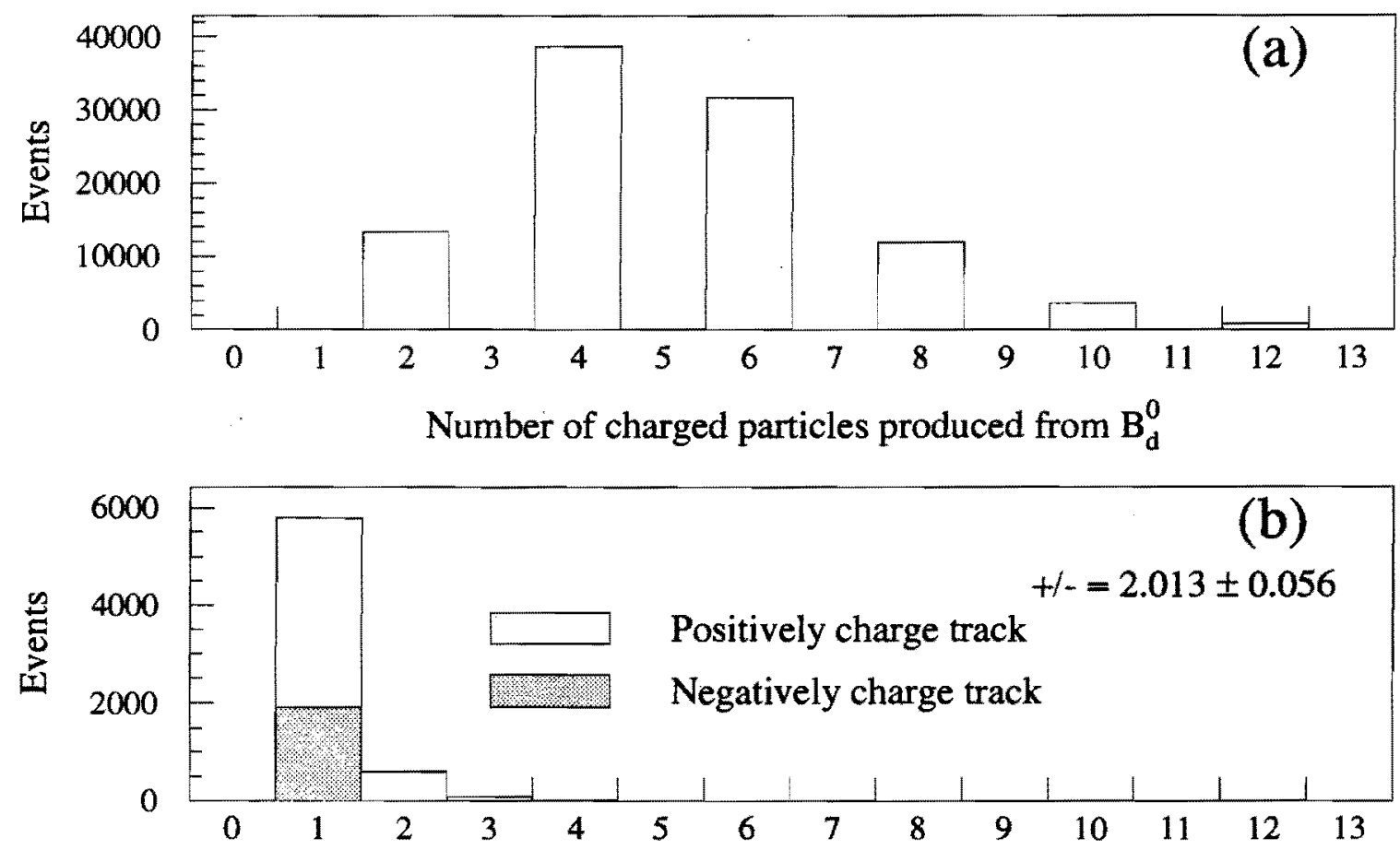

Number of charged tracks from $\mathrm{B}_{\mathrm{d}}^{0} ; \mathrm{P}_{\mathrm{T}}$ (track) $>3 \mathrm{GeV} / \mathrm{c}, \mid \eta($ track $) \mid<0.6$

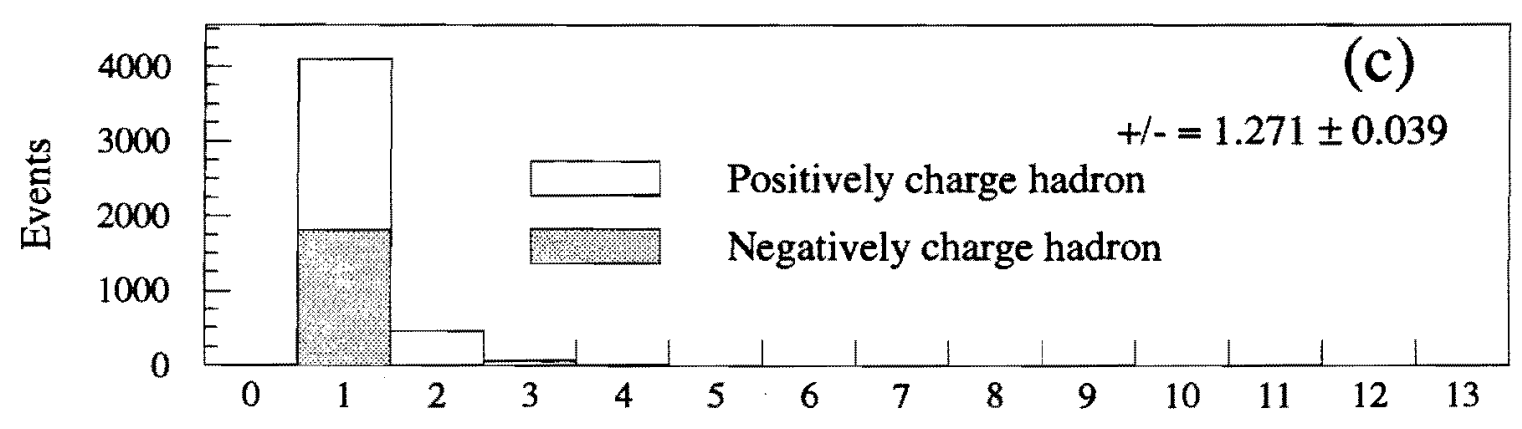

Number of charged hadrons from $\mathrm{B}_{\mathrm{d}}^{0} ; \mathrm{P}_{\mathrm{T}}$ (hadron) $>3 \mathrm{GeV} / \mathrm{c}$, $\mid \eta$ (hadron) $\mid<0.6$

Figure 5.12: Charged particle multiplicities from the $B_{d}^{0}$ meson. (a) Multiplicity of charged particles produced from $B_{d}^{0}$ mesons. (b) Multiplicity of charged tracks (hadron or lepton) with $P_{T}>3 \mathrm{GeV} / \mathrm{c}$ and $|\eta|<0.6$. (c) Multiplicity of charged hadrons with $P_{T}>3 \mathrm{GeV} / \mathrm{c}$ and $|\eta|<0.6$. The leptons are removed in this distribution. The hatched area in multiplicity one bin in (b) and (c) represents negatively charged tracks and hadrons. 

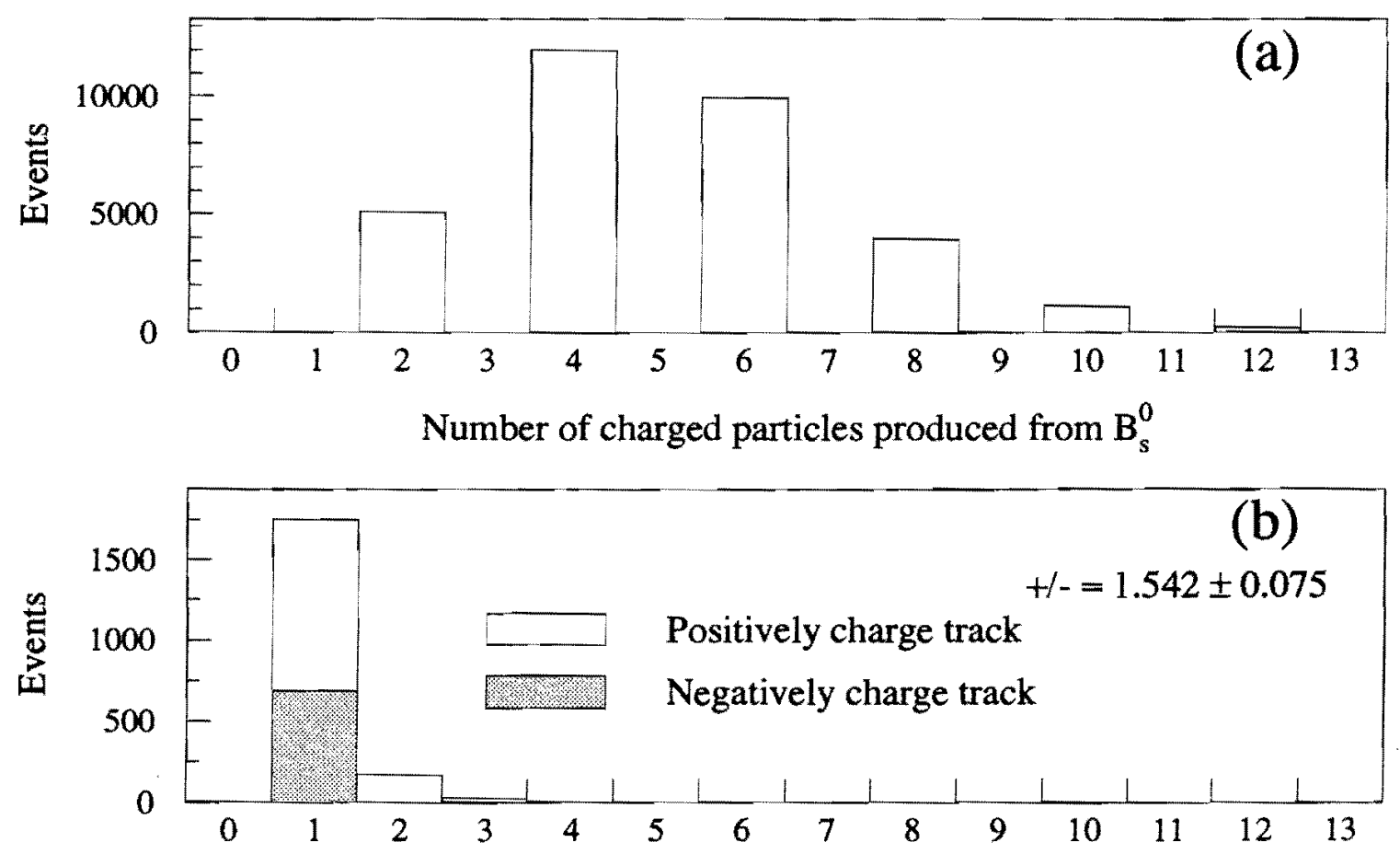

Number of charged tracks from $\mathrm{B}_{\mathrm{s}}^{0} ; \mathrm{P}_{\mathrm{T}}$ (track) $>3 \mathrm{GeV} / \mathrm{c}, \mid \eta$ (track) $\mid<0.6$

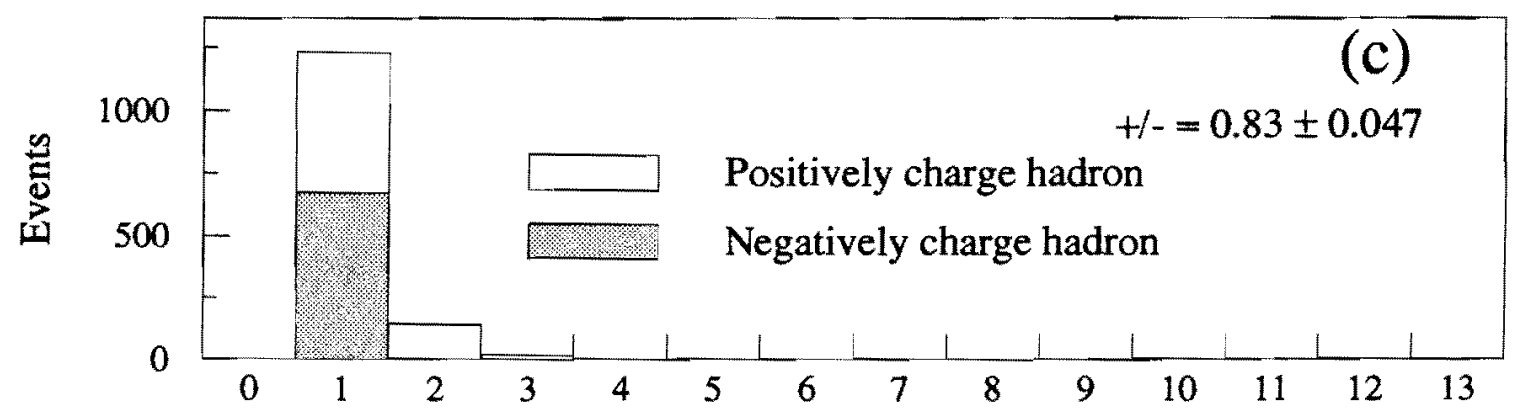

Number of charged hadrons from $\mathrm{B}_{\mathrm{s}}^{0} ; \mathrm{P}_{\mathrm{T}}$ (hadron) $>3 \mathrm{GeV} / \mathrm{c}$, $\mid \eta$ (hadron) $\mid<0.6$

Figure 5.13: Charged particle multiplicities from the $B_{s}^{0}$ meson. (a) Multiplicity of charged particles produced from $B_{s}^{0}$ mesons. (b) Multiplicity of charged tracks (hadron or lepton) with $P_{T}>3 \mathrm{GeV} / \mathrm{c}$ and $|\eta|<0.6$. (c) Multiplicity of charged hadrons with $P_{T}>3 \mathrm{GeV} / \mathrm{c}$ and $|\eta|<0.6$. The leptons are removed in this distribution. The hatched area in multiplicity one bin in (b) and (c) represents negatively charged tracks and hadrons. 


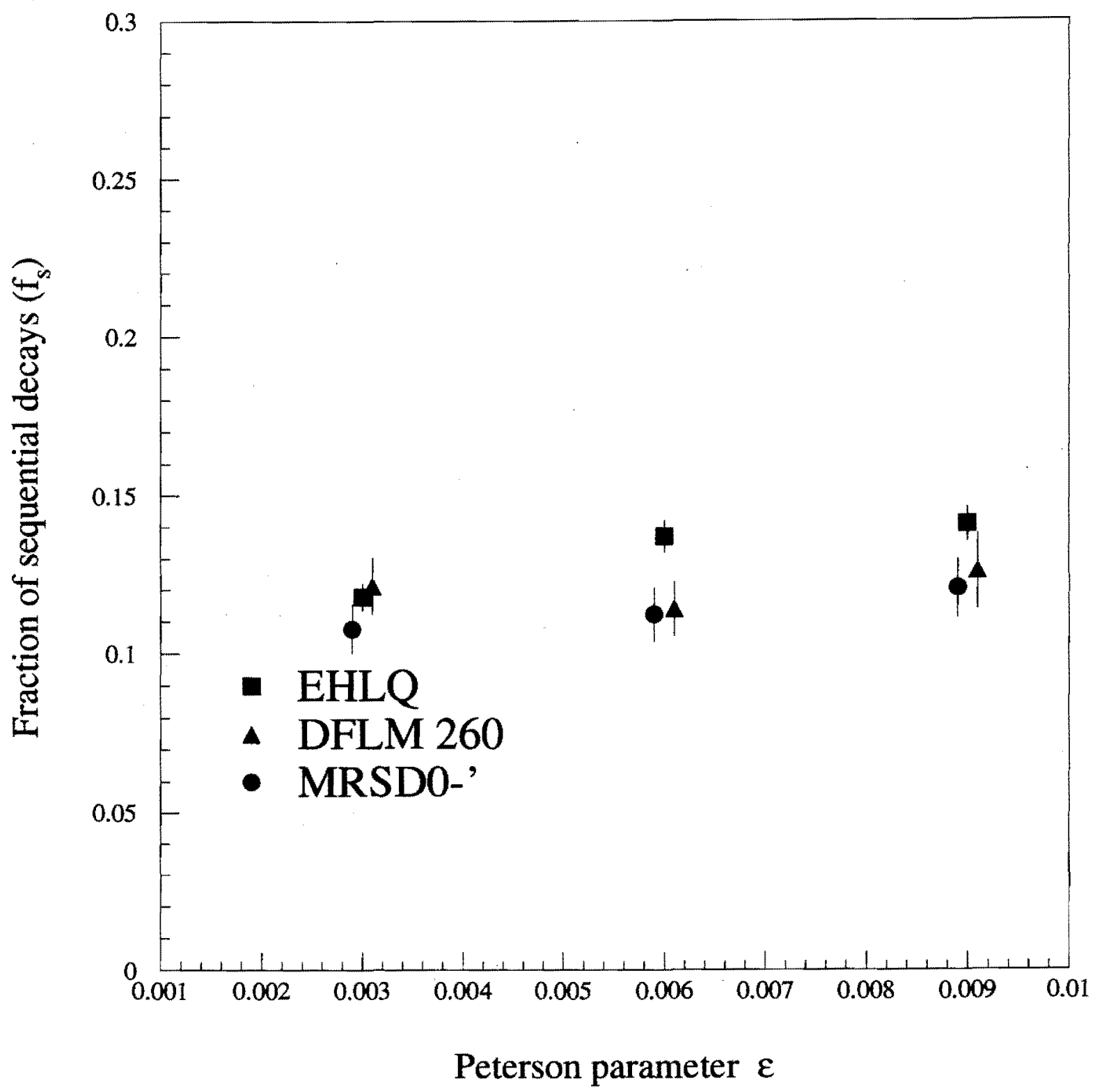

Figure 5.14: The fraction of the sequential decays for different Peterson parameter values and structure functions. 

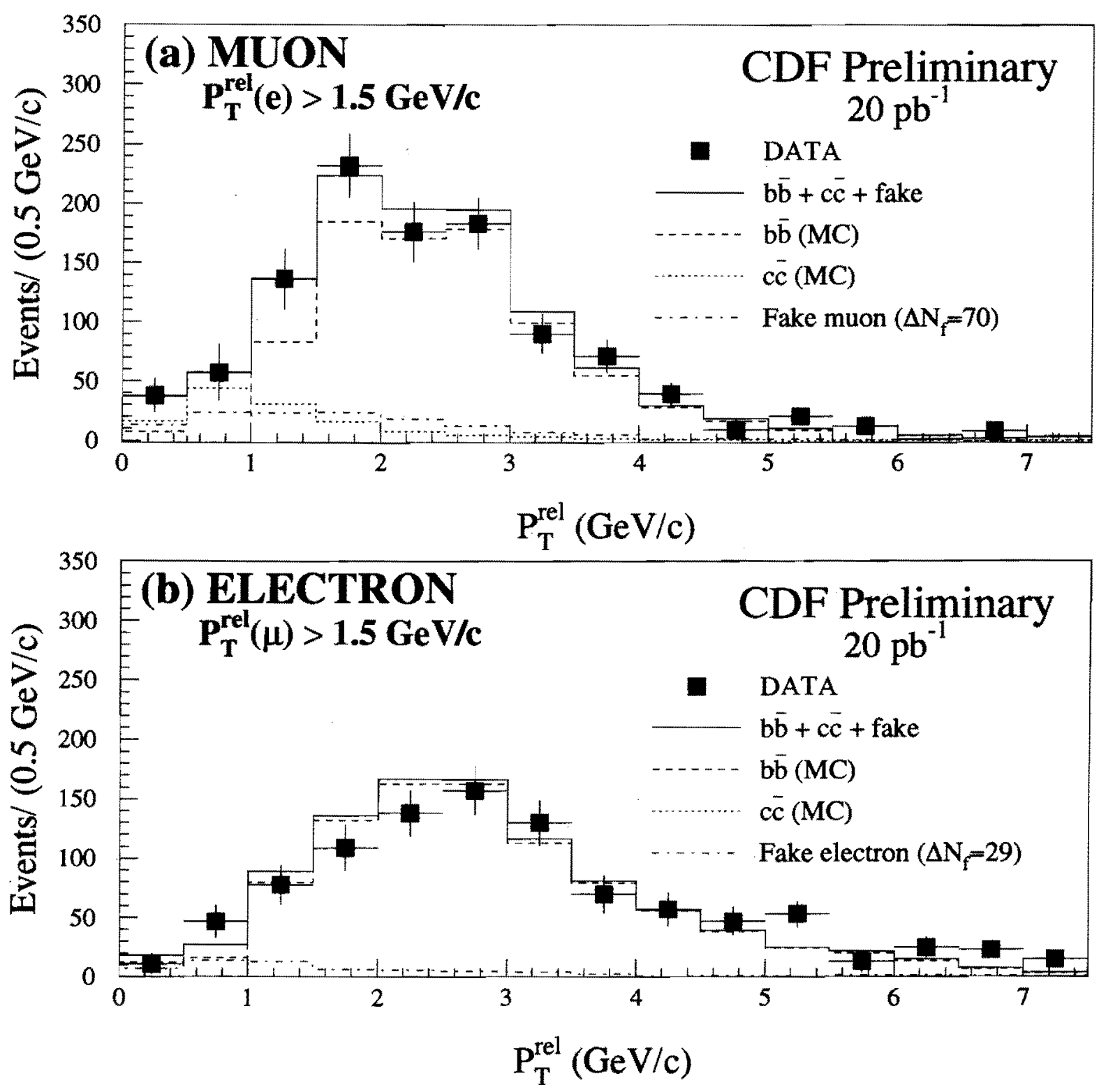

Figure 5.15: The difference between the numbers of OS and LS events as a function of $P_{T}^{\text {rel }}$ (a) for muons and (b) for electrons. The points are observed data. The bashed line represents $b \bar{b}$ Monte Carlo data, the dotted line $c \bar{c}$ Monte Carlo data, the dot-dashed line fake lepton events, the solid line the sum of these three. The Monte Carlo data are generated using MRSD0-' structure function set. The contributions from $c \bar{c}$ and fake lepton events are not clear by visible for the electron case. 

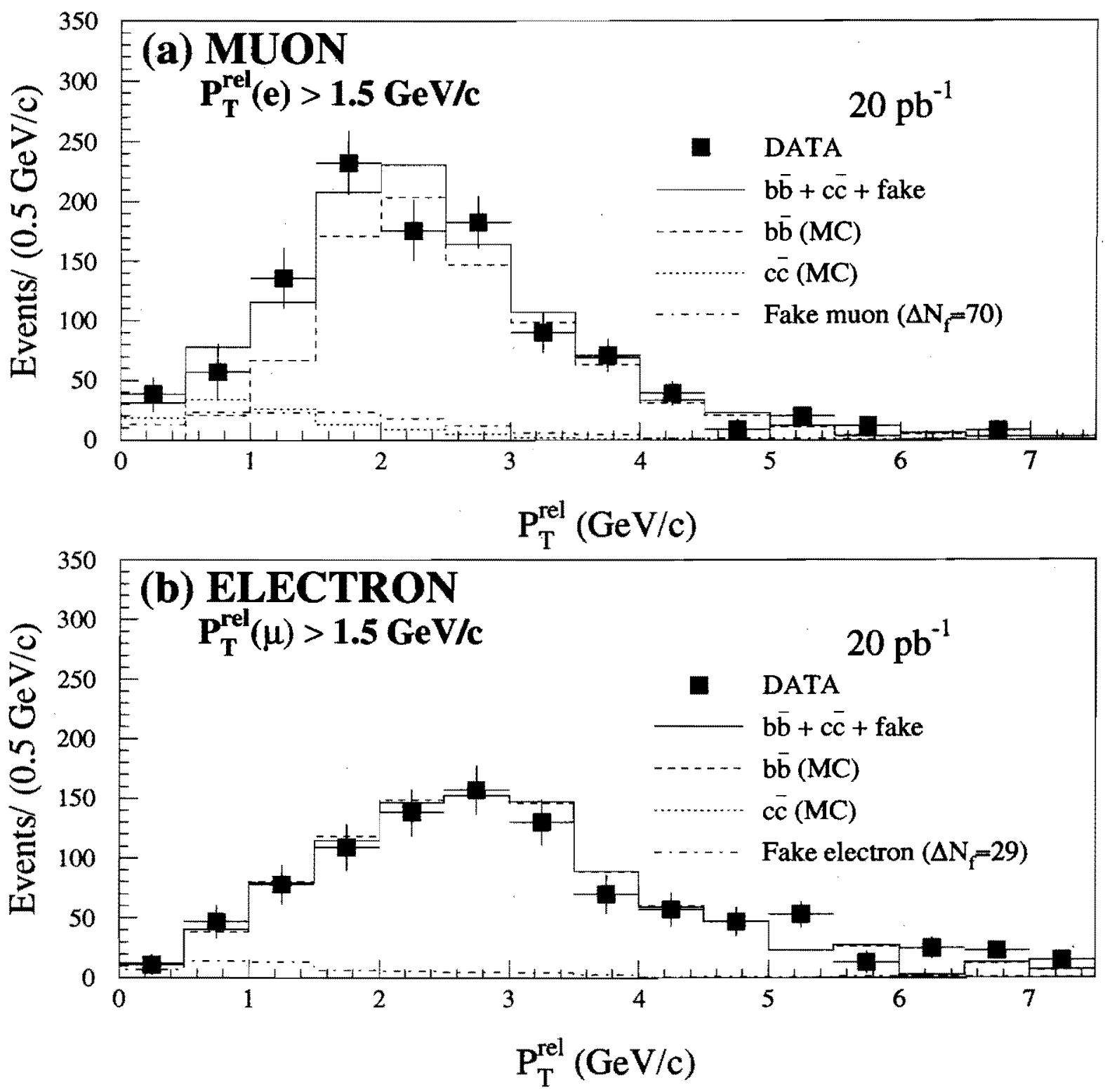

Figure 5.16: The difference between the numbers of OS and LS events as a function of $P_{T}^{r e l}$ (a) for muons and (b) for electrons. The points are observed data. The bashed line represents $b \bar{b}$ Monte Carlo data, the dotted line $c \bar{c}$ Monte Carlo data, the dot-dashed line fake lepton events, the solid line the sum of these three. The Monte Carlo data are generated using EHLQ structure function set. The contributions from $c \bar{c}$ and fake lepton events are not clear by visible for the electron case. 

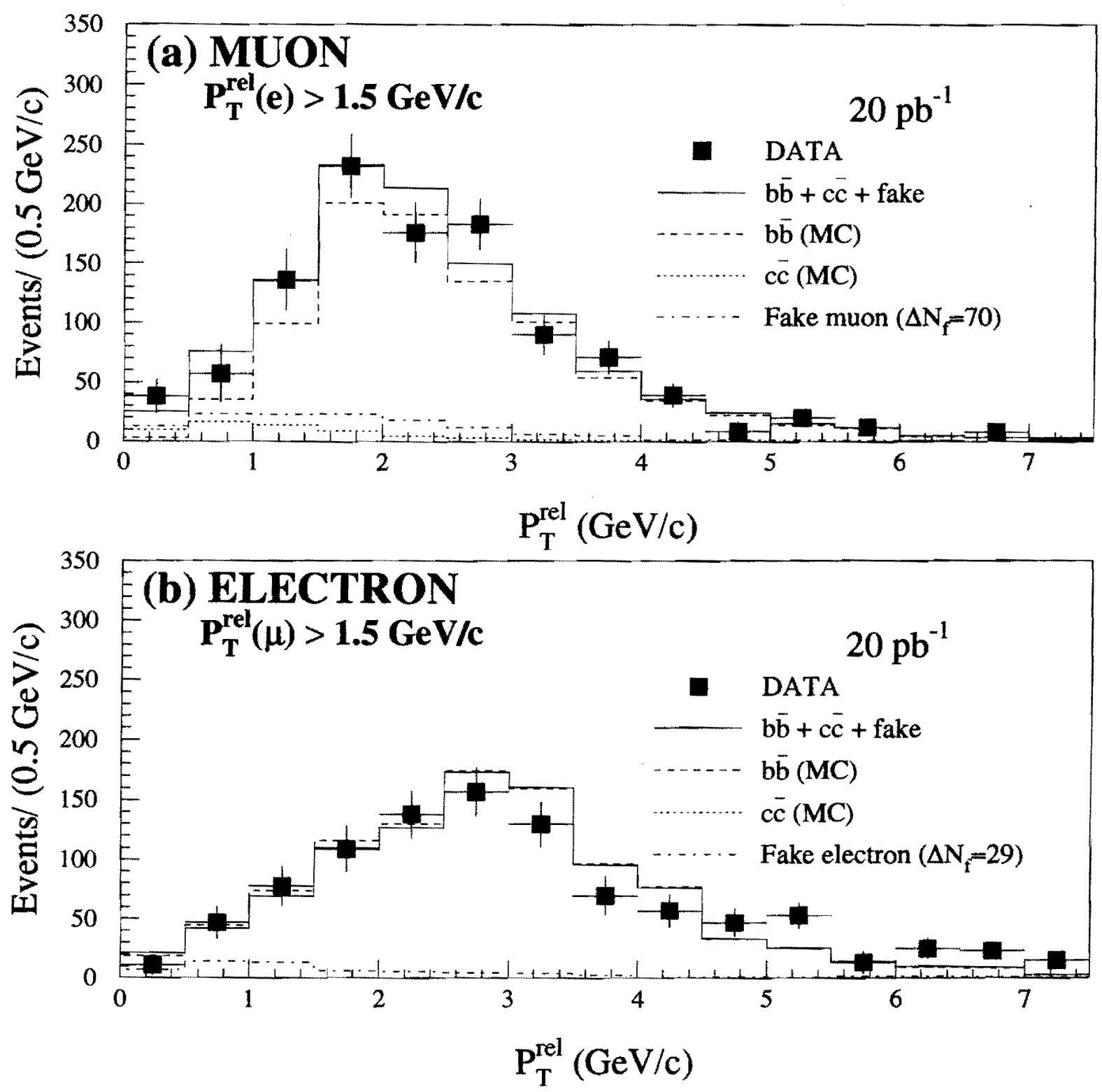

Figure 5.17: The difference between the numbers of OS and LS events as a function of $P_{T}^{r e l}$ (a) for muons and (b) for electrons. The points are observed data. The bashed line represents $b \vec{b}$ Monte Carlo data, the dotted line $c \bar{c}$ Monte Carlo data, the dot-dashed line fake lepton events, the solid line the sum of these three. The Monte Carlo data are generated using DFLM 260 structure function set. The contributions from $c \bar{c}$ and fake lepton events are not clear by visible for the electron case. 

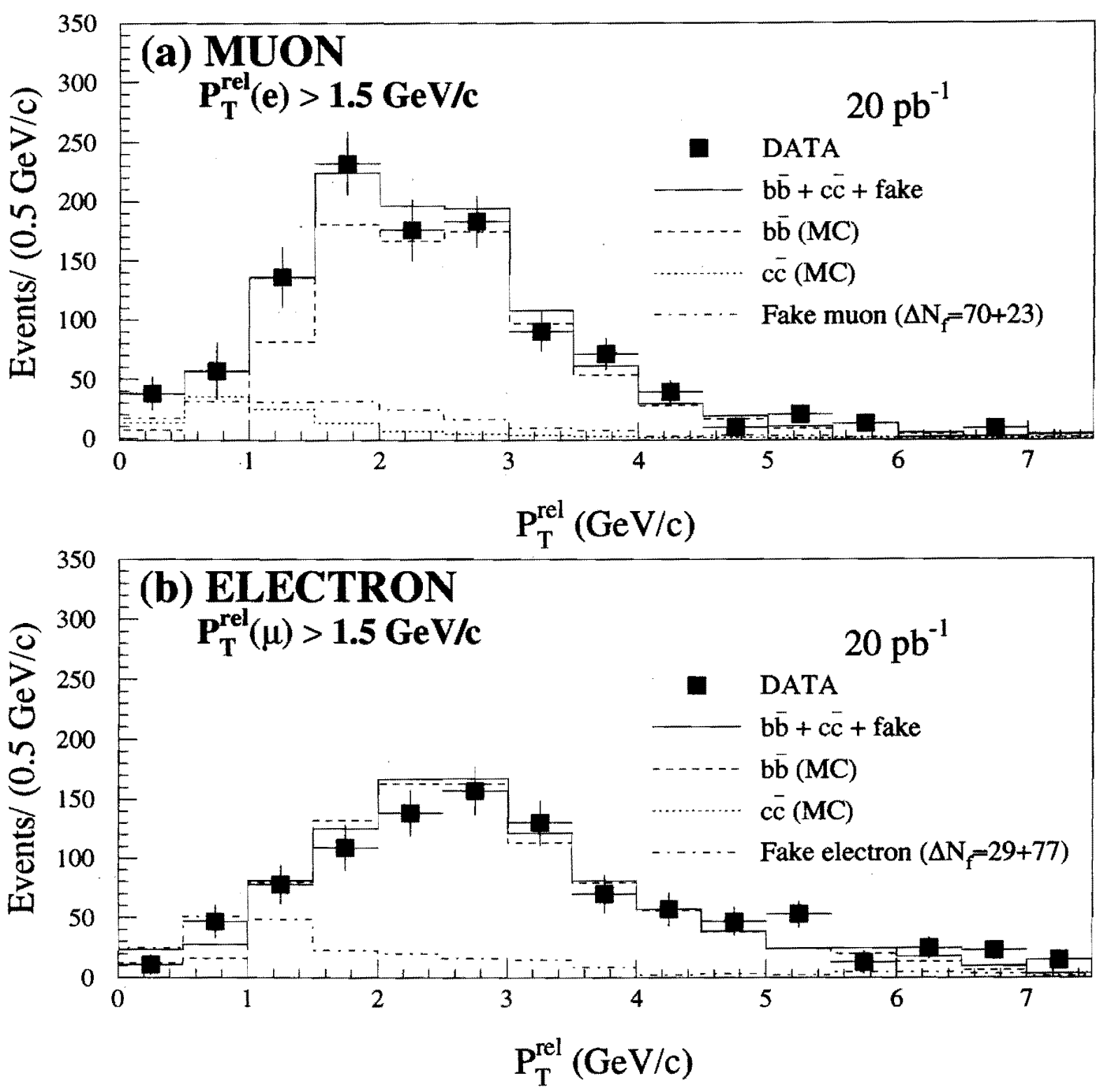

Figure 5.18: The difference between the numbers of OS and LS events as a function of $P_{T}^{\text {rel }}$ (a) for muons and (b) for electrons. The points are observed data. The bashed line represents $b \bar{b}$ Monte Carlo data, the dotted line $c \bar{c}$ Monte Carlo data, the dot-dashed line fake lepton events, the solid line the sum of these three. The Monte Carlo data are generated using MRSD0 ${ }^{\prime}$ structure function set. In this distributions, numbers of fake muons and electrons are normalized to $70+23$ and $29+77$, respectively. The contributions from $c \bar{c}$ and fake lepton events are not clear by visible for the electron case. 

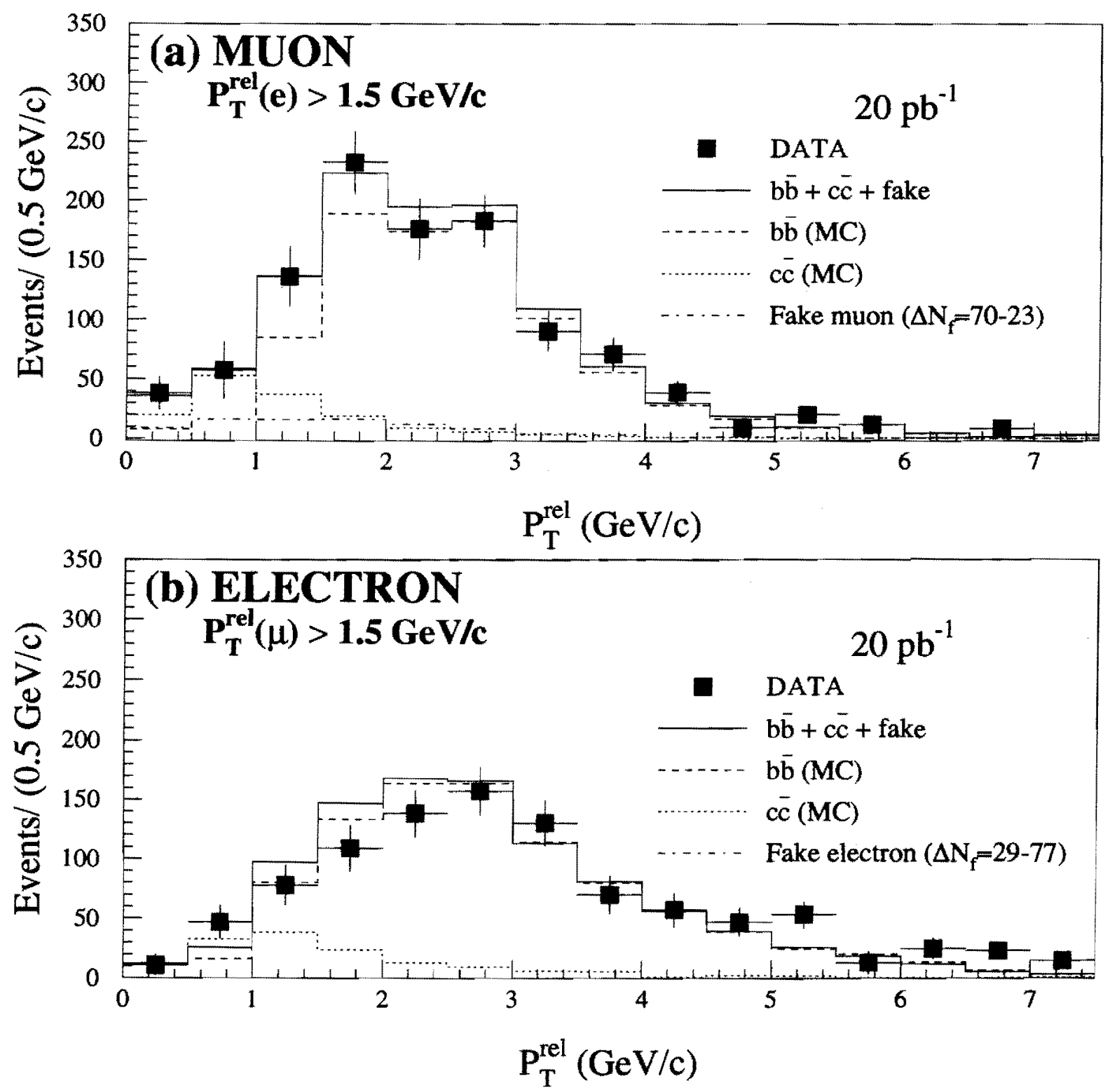

Figure 5.19: The difference between the numbers of OS and LS events as a function of $P_{T}^{\text {rel }}$ (a) for muons and (b) for electrons. The points are observed data. The bashed line represents $b \bar{b}$ Monte Carlo data, the dotted line $c \bar{c}$ Monte Carlo data, the dot-dashed line fake lepton events, the solid line the sum of these three. The Monte Carlo data are generated using MRSD0 ${ }^{\prime}$ structure function set. In this distributions, numbers of fake muons and electrons are normalized to $70-23$ and $29-77$, respectively. The contributions from $c \bar{c}$ and fake lepton events are not clear by visible for the electron case. 


\section{Chapter 6}

\section{Results and Discussion}

\subsection{Mixing parameter, $\chi$}

From Eqs. (4.2) and (4.3), the mixing parameter $\chi$ is related to the ratio $R$ of the number of LS events to that of OS events by

$$
R=\frac{N^{e x p}(L S)\left(1-F_{e \mu}(L S)\right)}{N^{\exp }(O S)\left(1-F_{e \mu}(O S)\right)}=\frac{2 \chi(1-\chi)+\left[(1-\chi)^{2}+\chi^{2}\right] f_{s}}{(1-\chi)^{2}+\chi^{2}+2 \chi(1-\chi) f_{s}+f_{c}}
$$

Substituting the numerical values, $F_{e \mu}(O S)=0.255 \pm 0.021, F_{e \mu}(L S)=0.395 \pm 0.031$, $f_{s}=0.137 \pm 0.027$ and $f_{c}=0.007 \pm 0.011$, together with the observed $N^{\exp }(O S)=1710$ and $N^{\exp }(L S)=861$, we obtained

$$
\chi=0.130 \pm 0.010(\text { stat }) \pm 0.010(\text { syst })
$$

The uncertainties in $\chi$ are shown in Table 6.1. The obtained result (Eq. (6.2)) is consistent with the 88/89 CDF result and also with other experiments as shown in Fig. 6.1. 


\begin{tabular}{l|c}
\hline Source & Uncertainties \\
\hline Statistical & 0.010 \\
Fake lepton events & 0.004 \\
Fraction of sequential decays & 0.009 \\
Fraction of $c \bar{c}$ events & 0.002 \\
\hline Total & 0.014 \\
\hline
\end{tabular}

Table 6.1: Uncertainties in $\chi$.

\subsection{Mixing parameter for $B_{s}$ mesons, $\chi_{s}$}

To obtain the value of $\chi_{s}$, we used the relation $\chi=P_{d} \chi_{d}+P_{s} \chi_{s}$. The $B_{d}^{0}$ and $B_{s}^{0}$ fractions, $P_{d}$ and $P_{s}$, are inferred from measurements of the relative production rates of kaons and pions. We have assumed $P_{d}=0.391$ and $P_{s}=0.117$. These values correspond to a strange quark suppression factor $P_{s} / P_{d}=0.3$ consistent with measurements at LEP [51] and lower energy $e^{+} e^{-}$colliders $[52,53]$. The mixing parameter, $\chi$, gives a constraint on the $\chi_{d^{-}} \chi_{s}$ plane as shown in Fig. 6.2. The dotted line represents the ARGUS and CLEO combined result of $\chi_{d}$ :

$$
\chi_{d}=0.15 \pm 0.03
$$

which was measured by ARGUS (1992 and 1994) and CLEO (1993) $[9,10,58]$. The shaded region is allowed by the standard model (see Eq. (2.19)). The $B_{s}^{0}$ meson is expected to be maximally mixed from the ARGUS \& CLEO results combined with the Standard Model prediction. Our result is consistent with this expectation.

We combined our result and the world average value in the Particle Data Book (1994) to obtain a new world average

$$
\chi=0.132 \pm 0.008
$$

Assuming that the errors in $\chi_{d}$ (Eq. (6.3)) and $\chi$ (Eq. (6.4)) are independent and rep- 
resent $1 \sigma$ uncertainty of the Gaussian error function, we derive a limit on $\chi_{s}$ :

$$
\chi_{s}>0.447 \quad(68.3 \% \text { C.L. }) .
$$

The contour in the $\chi_{s}-\chi_{d}$ plane is shown in Fig. 6.3.

The value of $\chi_{s}$ is sensitive to the relative production fractions of $B_{d}^{0}$ and $B_{s}^{0}$ mesons. The dependence of $\chi_{s}$ on $P_{s} / P_{d}$ is shown in Fig. 6.4, up to the $\mathrm{SU}(3)$ flavor symmetry limit of $P_{s} / P_{d}=1$. 


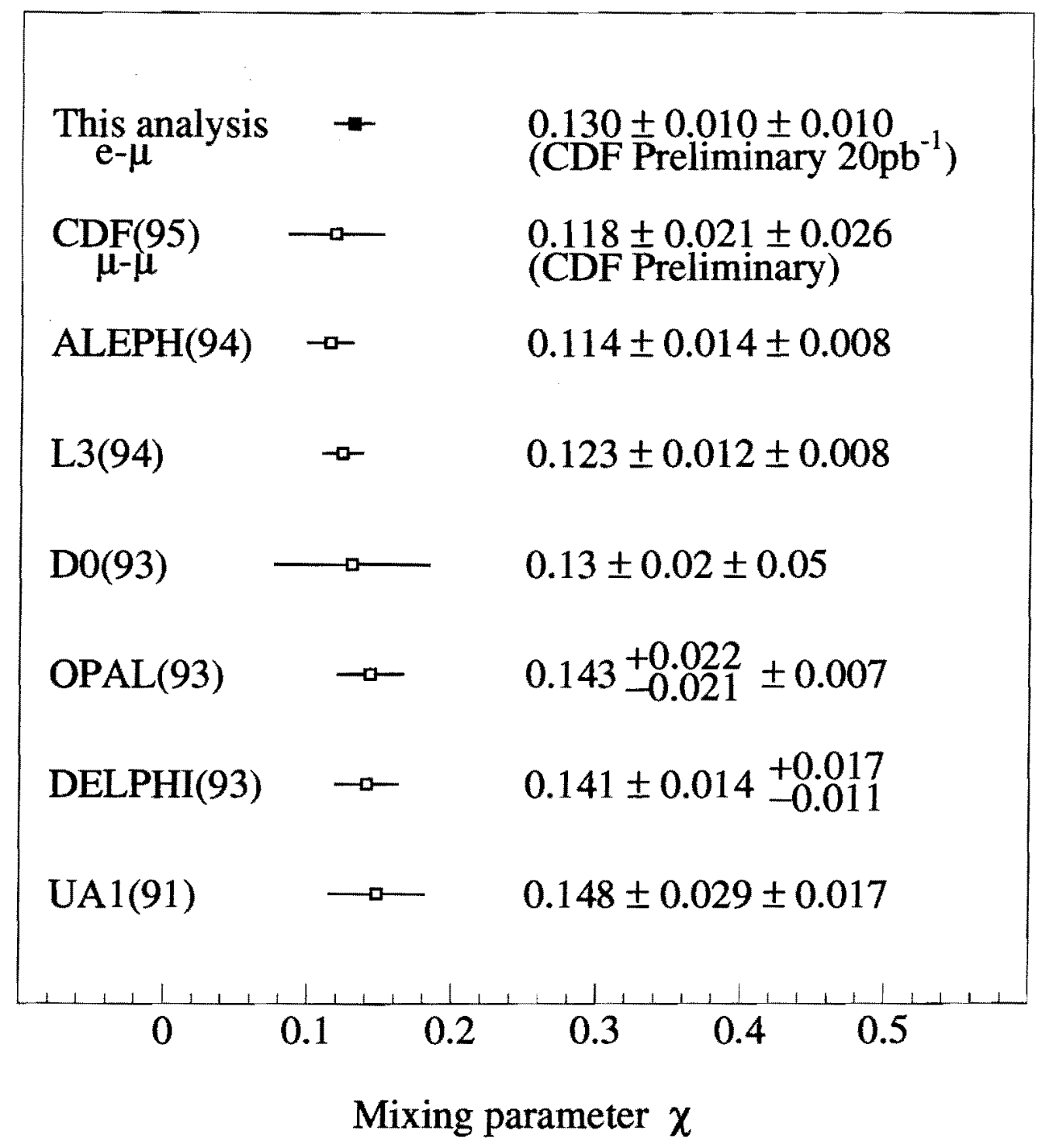

Figure 6.1: Comparison with other experiments. 


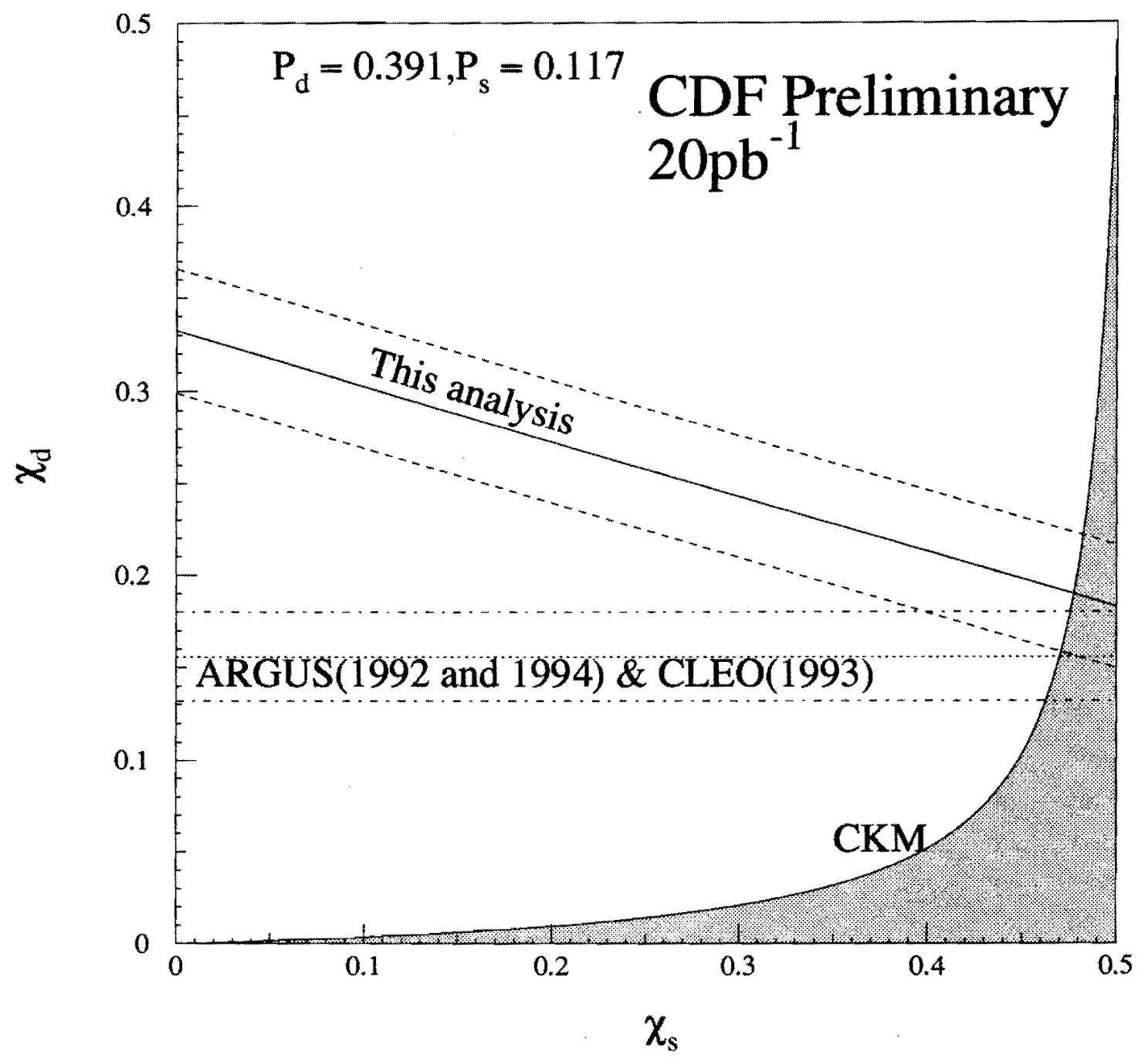

Figure 6.2: The mixing parameter for $B_{d}^{0}$ versus that for $B_{s}^{0}$ assuming that $P_{d}$ and $P_{s}$ are 0.391 and 0.117 . The $\chi_{d}$ range is the ARGUS (1992\&1994) and CLEO (1993) combined result of $0.15 \pm 0.03$. The bands represent $\pm 1 \sigma$ uncertainty. 


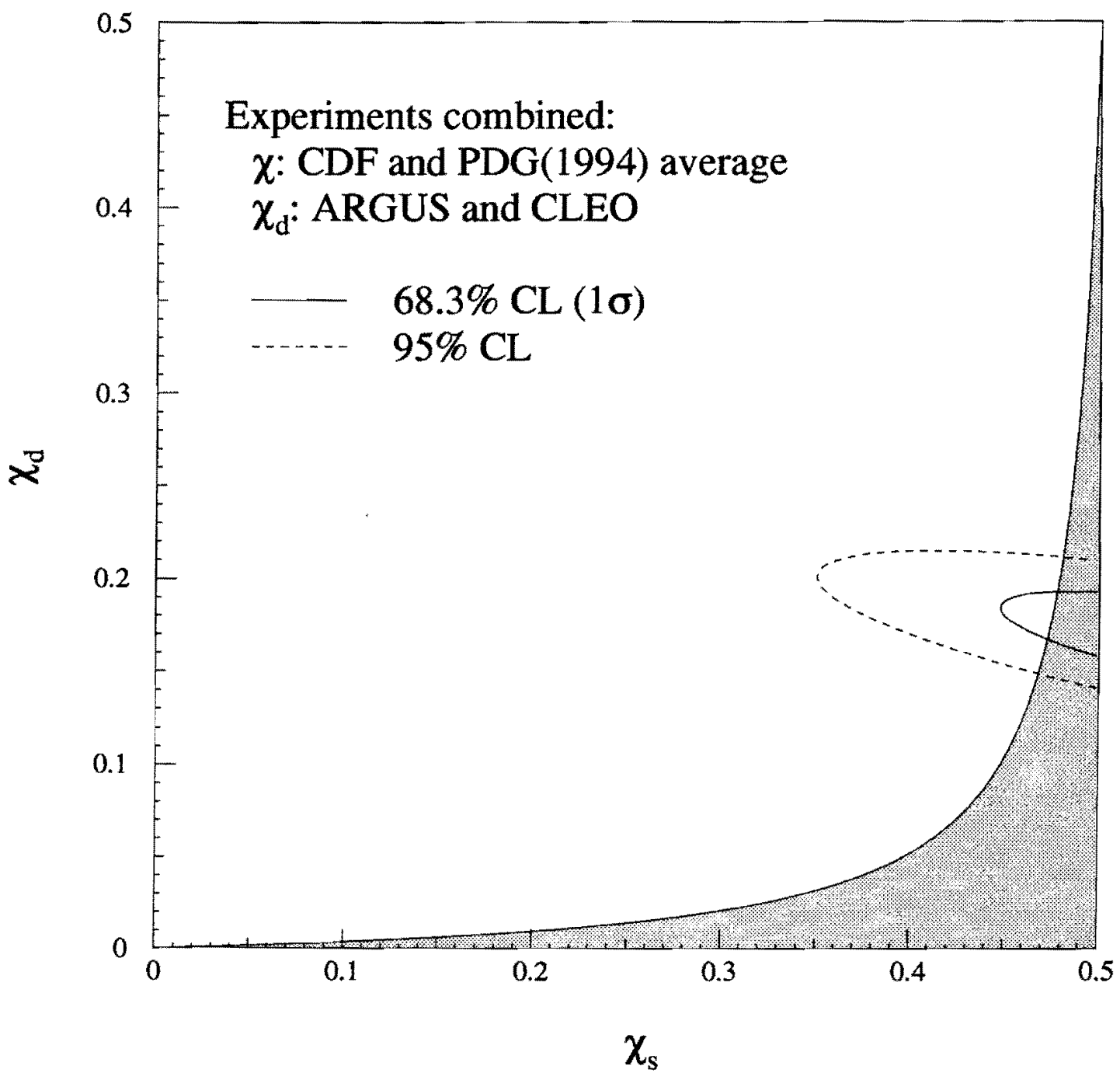

Figure 6.3: The mixing parameter for $B_{d}^{0}$ versus that for $B_{s}^{0}$ assuming $\boldsymbol{P}_{d}$ and $\boldsymbol{P}_{s}$ are 0.391 and 0.117 . The $\chi$ was calculated from our result and average value in the Particle Data Book. The $\chi_{d}$ is estimated by the ARGUS (1992\&1994) and CLEO (1993) combined result of $0.15 \pm 0.03$. The $68.3 \%$ (solid) and $95 \%$ (dashed) confidence areas are shown. 


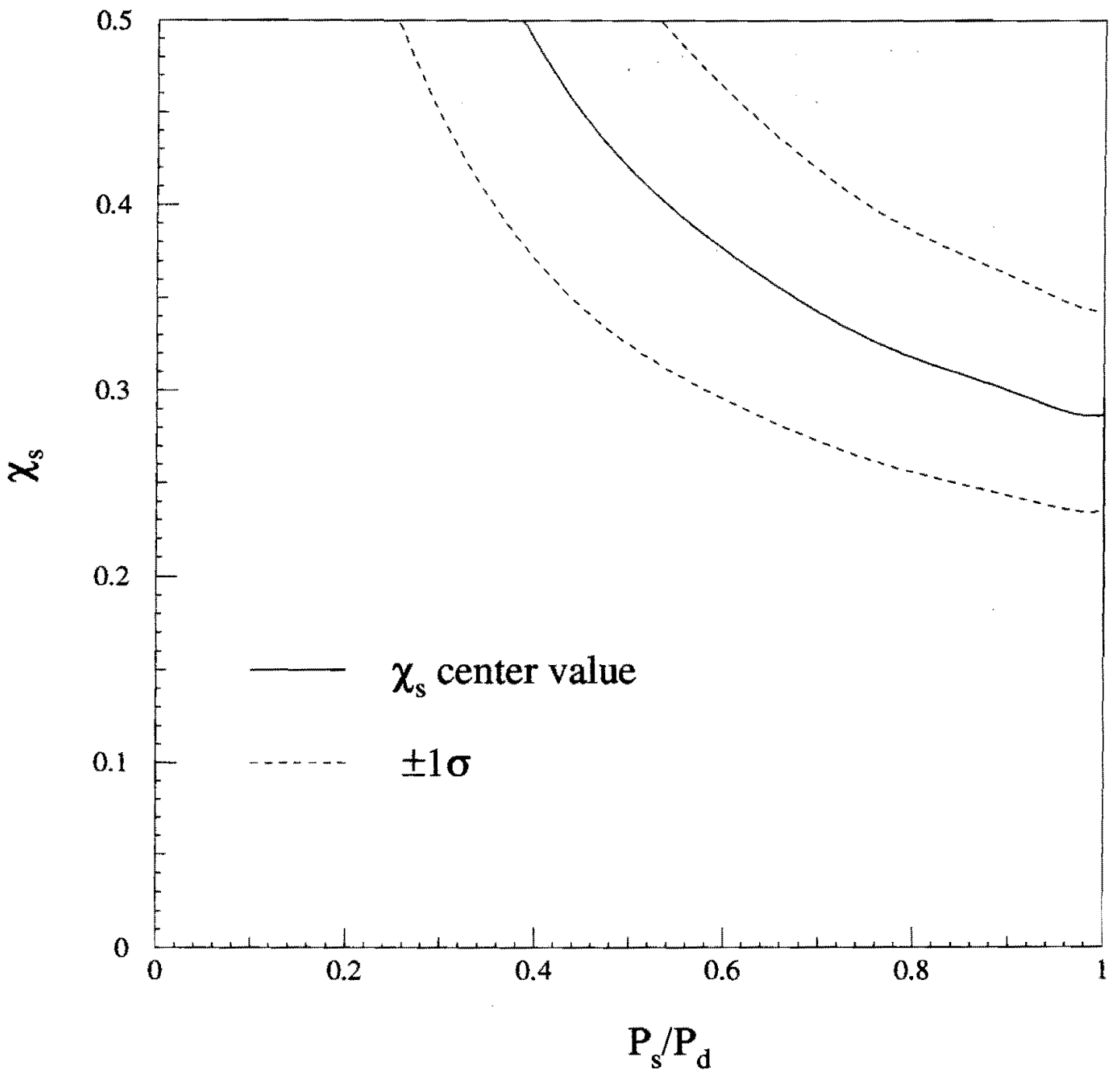

Figure 6.4: $\chi_{s}$ as a function of $P_{s} / P_{d}$. 


\section{Chapter 7}

\section{Conclusion}

We measured $B^{0} \bar{B}^{0}$ mixing parameter, $\chi$, in electron muon events in proton antiproton collisions at $\sqrt{s}=1.8 \mathrm{TeV}$ using the CDF detector. The data were collected in the 1992-1993 CDF run. The corresponding integrated luminosity was $20 \mathrm{pb}^{-1}$. We have exploited the good electron and muon identification capabilities of the CDF detector to measure the $B^{0} \bar{B}^{0}$ mixing parameter. We obtained the mixing parameter as

$$
\chi=0.130 \pm 0.010(\text { stat. }) \pm 0.010(\text { syst. }) .
$$

This result improves the previous CDF result by a factor of 3 and is one of the most accurate measurements of $\chi$ at the present time. The result is consistent with other measurements of $\chi$ and also with the expectations from the standard model using other experimental results.

Since both neutral $B$ mesons, $B_{d}^{0}$ and $B_{s}^{0}$, are produced in $p \bar{p}$ collisions, the mixing parameter for $B_{s}^{0}, \chi_{s}$, is estimated by using the mixing parameter for $B_{d}^{0}$ measured by the ARGUS and the CLEO groups in $e^{+} e^{-}$collisions and by assuming the fractions of $B_{d}^{0}$ and $B_{s}^{0}$ mesons produced in $b \bar{b}$ events. The value of the mixing parameter for $B_{s}$ mesons is consistent with the expectations from the standard model. 


\section{Appendix A}

\section{Estimation of Probabilities for Real}

\section{Leptons from $J / \psi \rightarrow l l$ Events}

In this appendix, we derive Eq. (5.9) used to estimate the $P_{r}$.

Before to discuss $J / \psi \rightarrow l l$ events, We first consider the definition of the probability, $P_{r}$, for a single lepton. Let $N_{r}$ be the total number of real leptons. Call $N_{t}$ the number of leptons which pass the original identification cut, and $N_{l}$ the number of leptons which pass the loose identification cut. We write $\epsilon_{t}$ for the probability that the lepton passed the original cut, and $\epsilon_{l}$ for the probability that leptons which fail the original cut, pass the loose cut. The numbers $N_{t}$ and $N_{l}$ are related to $N_{r}, \epsilon_{t}$ and $\epsilon_{l}$ by

$$
\begin{aligned}
N_{t} & =N_{r} \epsilon_{t} \\
N_{l} & =N_{t}+N_{r}\left(1-\epsilon_{t}\right) \epsilon_{l} \\
& =\left[\epsilon_{t}+\left(1-\epsilon_{t}\right) \epsilon_{l}\right] N_{r}
\end{aligned}
$$

Since the probability $P_{r}$ is defined by a ratio of number of leptons which pass the original cut to number of leptons which pass the loose cut, the $P_{r}$ can be written by

$$
P_{r}=\frac{N_{t}}{N_{l}}
$$




$$
=\frac{\epsilon_{t}}{\epsilon_{t}+\left(1-\epsilon_{t}\right) \epsilon_{l}}
$$

When $N$ is the number of $J / \psi \rightarrow l l$ events produced, let $N_{1}$ be the number of $J / \psi$ 's for which at least one lepton passes the original identification cut, and $N_{2}$ be the number of $J / \psi$ 's for which both leptons pass the cut. We write the $N_{1}$ and $N_{2}$ using $\epsilon_{t}$ and $\epsilon_{l}$ as

$$
\begin{aligned}
& N_{1}=N \epsilon_{t}^{2}+2 N \epsilon_{t}\left(1-\epsilon_{t}\right) \epsilon_{l} \\
& N_{2}=N \epsilon_{t}^{2}
\end{aligned}
$$

From these equations and Eq. (A.5), we get.

$$
P_{r}=\frac{2 N_{2}}{N_{1}+N_{2}}
$$




\section{Appendix B}

\section{Uncertainty in $f_{s}$ due to Monte}

\section{Carlo Statistics, Structure Function}

\section{and Peterson Parameter}

The uncertainty in $f_{s}$ due to Monte Carlo statistics, structure functions and Peterson fragmentation parameter was estimated by using minimum and maximum of center values for each Monte Carlo data set. Is this estimation reasonable?

The center values and statistical uncertainties of $f_{s}$ for different structure function sets and Peterson parameters are listed in Table B.1. We made the probability distributions of $f_{s}$ using the center value and uncertainties assuming the Gaussian distributions where the center value and the statistical uncertainty were used as the mean value and sigma of the Gaussian distribution. The probability distributions for different structure function sets and Peterson parameters are shown in Fig. B.1.

We assume that each of the nine probability distributions represent and independent measurement of $f_{s}$. Then we make the sum of the nine distributions to determine the center value and its error (Fig. B.2). The sum probability distribution is fitted by a Gaussian function and the results are listed in the figure. From the results, the relative uncertainty in $f_{s}$ due to structure functions, Peterson parameter and Monte 


\begin{tabular}{c|c||c|c}
\hline \hline Structure function & Peterson parameter $\epsilon$ & $f_{s}$ & Statistical uncertainty \\
\hline & 0.003 & 0.1055 & 0.0075 \\
MRSD0-' & 0.006 & 0.1107 & 0.0084 \\
& 0.009 & 0.1190 & 0.0092 \\
\hline & 0.003 & 0.1158 & 0.0041 \\
EHLQ & 0.006 & 0.1355 & 0.0050 \\
& 0.009 & 0.1390 & 0.0053 \\
\hline & 0.003 & 0.1196 & 0.0089 \\
DFLM 260 & 0.006 & 0.1124 & 0.0084 \\
& 0.009 & 0.1242 & 0.0118 \\
\hline \hline
\end{tabular}

Table B.1: Fraction of sequential decays with MRSD0-', EHLQ and DFLM 260 structure functions and, with Peterson parameter $\epsilon=0.003,0.006,0.009$.

Carlo statistics is $10.2 \%$. This is consistent with the uncertainty which is estimated in Section 5.2.4. 


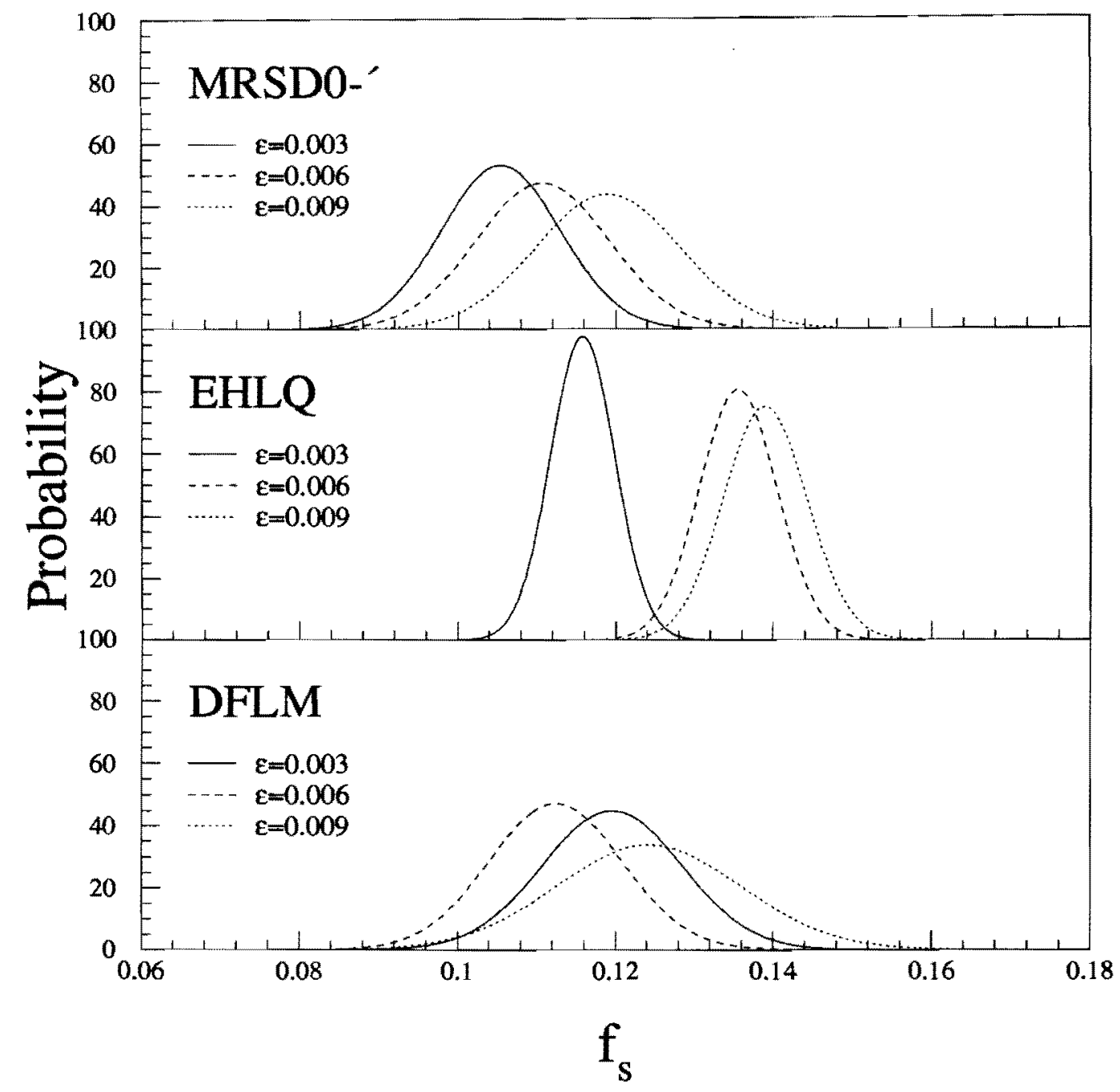

Figure B.1: The probability distributions of $f_{s}$. The structure functions for the top, middle and bottom figures are MRSD0-', EHLQ and DFLM, respectively. In each figure, the solid (dashed or dotted) line represents the probability with Peterson fragmentation parameter $\epsilon=0.003(0.006$ or 0.009$)$. 


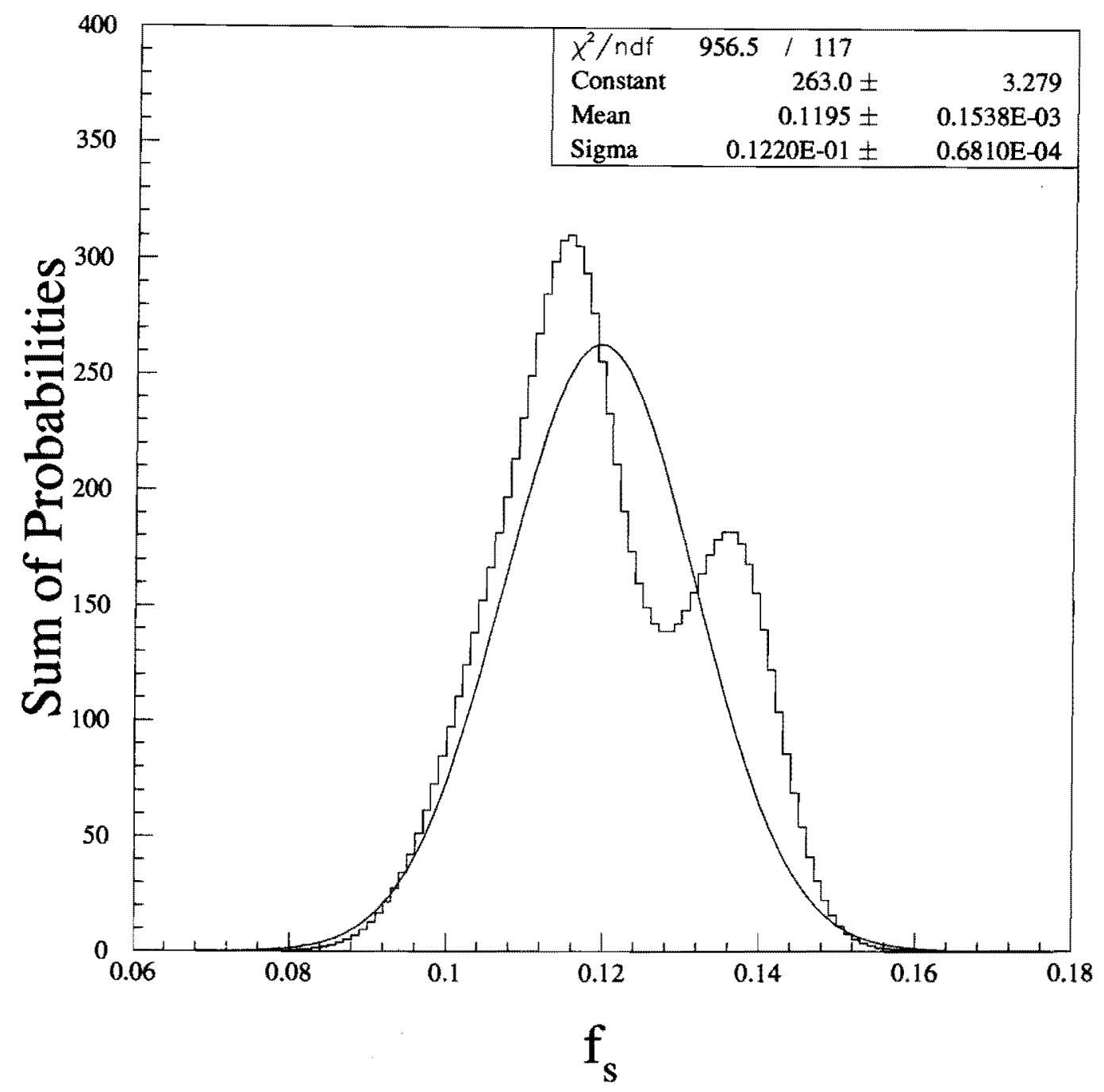

Figure B.2: The sum of probability distributions for $f_{s}$. The distribution is fitted by a Gaussian distribution. The fitted parameters of the Gaussian distribution are listed in the figure. 


\section{Bibliography}

[1] F. Abe et al. (CDF collab.), Phys. Rev. Lett. 74, 2626, (1995)

[2] S. Abachi et al. (D0 collab.), Phys. Rev. Lett. 74, 2632, (1995)

[3] M. Kobayashi and T. Maskawa, Prog. Theor. Phys. 49, 652 (1973).

[4] Particle Data Group, Review of Particle Properties, Phys. Rev. D50, 1173 (1994).

[5] L. Wolfenstenin, Phys. Rev. Lett. 51, 1945 (1983)

[6] Sheldon Stone, "B DECAYS", 460 (1994).

[7] J. Chrin, Z. Phys. C36, 165 (1987).

[8] P. J. Franzini, Phys. Rept. 1731 (1989).

[9] H. Albrecht et al. (ARGUS collab.), Phys. Lett. B324, 249 (1994).

[10] J. Bartelt et al. (CLEO collab.), Phys. Rev. Lett. 71, 1680 (1993).

[11] D. Buskulic et al. (ALEPH collab.), Z. Phys. C62 179 (1994).

[12] M. Acciarri et al. (L3 collab.), Phys. Lett. B335 542 (1994).

[13] S. Abachi et al. (D0 collab.), Proceedings of the 9th Topical Workshop on ProtonAntiproton Collider Physics, Tsukuba, Japan (1993).

[14] P. D. Acton et al. (OPAL collab.), Phys. Lett. B276, 379 (1992). 
[15] P. Abreu et al. (DELPHI collab.), Phys. Lett. B301, 145 (1993).

[16] C. Albajar et al. (UA1 collab.), Phys. Lett. B262, 171 (1991).

[17] F. Abe et al. (CDF collab.), Phys. Rev. Lett. 67, 3351 (1991).

[18] D. Amidei et al., Nucl. Instrum. Methods A350, 73, (1994).

[19] F. Bedeschi et al., Nucl. Instrum. Methods A268, 50 (1988).

[20] L. Balka et al., Nucl. Instrum. Methods A267, 272 (1988).

[21] L. Balka et al., Nucl. Instrum. Methods A267, 272 (1988).

[22] S. Bertolucci et al., Nucl. Instrum. Methods A267, 301 (1988).

[23] S. Bertolucci et al, Nucl. Instrum. Methods A267, 301 (1988).

[24] Y. Fukui et al., Nucl. Instrum. Methods A267, 280 (1988).

[25] F. Abe et al., Nucl. Instrum. Methods A271, 387 (1988).

[26] Y. Fukui et al, Nucl. Instrum. Methods A267, 280 (1988).

[27] W. C. Carithers et al., "Performance and calibration studies of the CDF endplug hadron calorimeter", CDF note 368 .

[28] G. Ascoli et al., Nucl. Instrum. Methods A268, 33 (1988).

[29] A. Gauthier et al., "Design and performance of drift chambers for the central muon upgrade of the CDF detector", CDF internal note 1500 , to be submitted to Nucl. Instrum. Methods.

[30] D. Amidei et al., Nucl. Instrum. Methods A269, 51 (1988).

[31] G. Ascoli et al., Nucl. Instrum. Methods A269, 63 (1988).

[32] J. T. Carrol et al., Nucl. Instrum. Methods A300, 552 (1991). 
[33] G. W. Foster et al., Nucl. Instrum. Methods A269, 93 (1988).

[34] E. Barsotti et al., Nucl. Instrum. Methods A269, 82 (1988).

[35] G. Drake et al., Nucl. Instrum. Methods A269, 68 (1988).

[36] H. Brafman et al., IEEE Trans. Nucl. Sci. NS-32, 336 (1985).

[37] M. Bozzo et al., Phys. Lett. 147B, 392 (1984).

[38] M. Bozzo et al., Phys. Lett. 198B, 583 (1987).

[39] F. Abe et al., Phys. Rev. D44, 29 (1991).

[40] F. Abe et al., Phys. Rev. D50, 5550 (1994).

[41] F. E. Paige and S. D. Protopopescu, "ISAJET 5.20: A Monte Carlo event generator for $p \bar{p}$ and $p \bar{p}$ interactions.", BNL Report No. BNL-38034, 1986 (unpublished). ISAJET version 7.06 (1994) was used, specially modified fro the CDF analysis system and modified to include the parton distribution functions, using the CERN PDFLIB interface package from ref [54].

[42] Paul Avery, Ken Read and Garry Trahern, "QQ: A Monte Carlo Generator", CLEO Software Note CSN-212, March 25, 1985.

[43] Jonathan Lewis and Paul Avery, "CLEOMC: THe CDF Interface to the CLEO Monte Carlo (QQ)", CDF internal note 2724, 1994.

[44] ISALEP is a special version of ISAJET which is modified to generate heavy quark events with high- $P_{T}$ leptons effectively.

[45] M. Shapiro et al., "A User's Guide to QFL", CDF internal note 1810.

[46] N. Solomey and A. B. Wicklund, "Study of Electromagnetic Showers In the CDF Central Electromagnetic", CDF internal note 247 (1984). 
[47] D.A. Smith and H.B. Jensen, "Pion Punchthrough Probability of the Central Calorimeter Wedges", CDF internal note 707 (1988).

[48] F. Abe et al. (CDF collab.), Phys. Rev. D40, 3791 (1989).

[49] G.J. Alner et al. (UA5 collab.), Nucl. Phys. B258, 505 (1985).

[50] P. Kesten, L. Kirsch, and S. Tarem, "A measurement of $B \bar{B}$ Mixing Using Dielectrons", CDF internal note 1314 (1991).

[51] B. Adeva et al. (OPAL collab.), Phys. Lett. B264, 467 (1991).

[52] W. Bartel et al. (JADE collab.), Z. Phys. C20, 187 (1983).

[53] M. Althoff et al. (TASSO collab.), Z. Phys. C27, 27 (1985).

[54] H. Plothow-Besch, "PDFLIB: a library of all available parton density functions of the nucleon, the pion and the photon and the corresponding $\alpha_{s}$ calculations", Comp. Phys. Comm. 75, 396 (1993) This reference provides the interface to the parton distribution functions found in Ref. [57] and [55].

[55] A.D. Martin, R. G. Roberts and W. J. Stirling, Phys. Lett. B306, 145 (1993) and Phys. Lett. B309, 492 (1993).

[56] E. Eichiten, I. Hinchliffe, K. Lane and C. Quigg, Rev. Mod. Phys. 56, 579 (1984) and Rev. Mod. Phys. 58, 1065 (1985).

[57] M. Diemoz, F. Ferroni, E. Longo and G. Martinelli, Z. Phys. C39, 21 (1988)

[58] H. Albrecht et al. (ARGUS collab.), Z. Phys. C55, 357 (1992). 